\title{
Water-Quality Synoptic Sampling, July 1999: North Fork Shenandoah River, Virginia
}

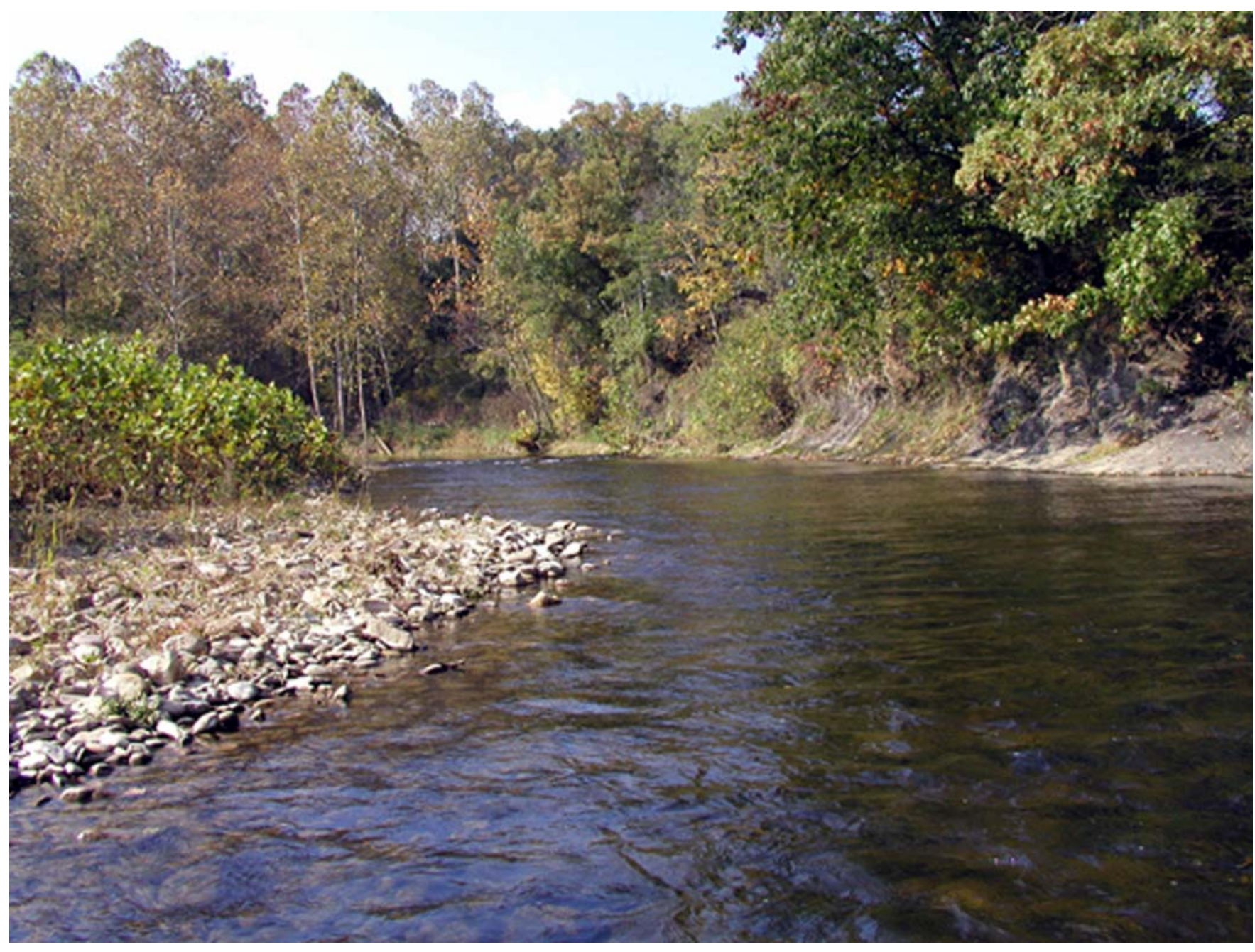

Scientific Investigations Report 2004-5153 
Cover photograph of North Fork Shenandoah River, near Cootes Store, Virginia; view looking upstream, by Donald C. Hayes, U.S. Geological Survey, October 22, 2002. 


\section{Water-Quality Synoptic Sampling, July 1999: North Fork Shenandoah River, Virginia}

By Jennifer L. Krstolic and Donald C. Hayes

Northern Shenandoah Valley Regional Commission and Virginia Polytechnic Institute and State University

Scientific Investigations Report 2004-5153 


\title{
U.S. Department of the Interior Gale A. Norton, Secretary
}

\author{
U.S. Geological Survey \\ Charles G. Groat, Director
}

U.S. Geological Survey, Reston, Virginia: 2004

For more information about the USGS and its products:
Telephone: 1-888-ASK-USGS
World Wide Web: http://www.usgs.gov/
Any use of trade, product, or firm names in this publication is for descriptive purposes only
and does not imply endorsement by the U.S. Government.

For additional information write to:
District Chief
U.S. Geological Survey
1730 East Parham Rd.
Richmond, VA 23228
dc_va@usgs.gov
Information about water resources in Virginia is available online at http://va.water.usgs.gov 


\section{Contents}

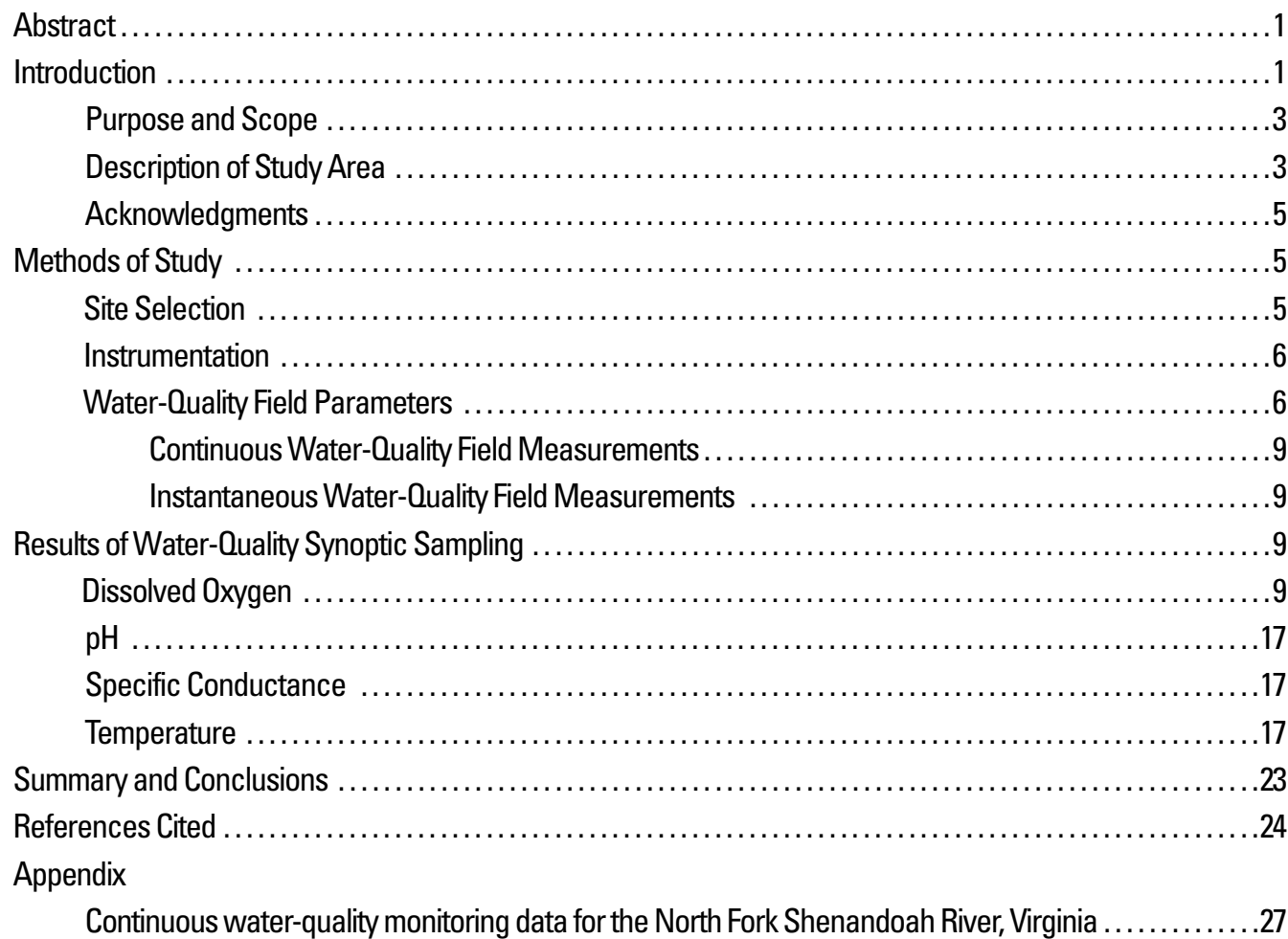

\section{Figures}

1-2. Maps showing:

1. Location of the North Fork Shenandoah River Basin, Virginia $\ldots \ldots \ldots \ldots \ldots \ldots \ldots \ldots \ldots \ldots \ldots \ldots \ldots \ldots \ldots \ldots \ldots \ldots \ldots \ldots$

2. Locations of continuous and instantaneous water-quality monitoring sites, and streamflow-gaging stations on the North Fork Shenandoah River, Virginia $\ldots \ldots \ldots \ldots \ldots \ldots . \ldots$

3-10. Graphs showing:

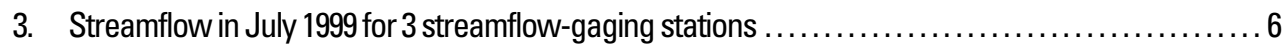

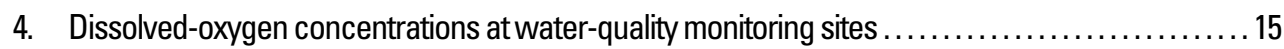

5. Dissolved-oxygen as percent air saturation at water-quality monitoring sites ................ 16

6. Ratios of dissolved-oxygen concentrations at water-quality monitoring sites ................ 18

7. Dissolved-oxygen concentrations at three pairs of upstream and

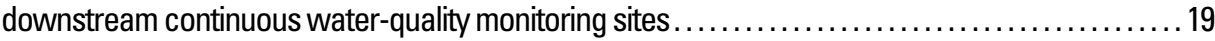

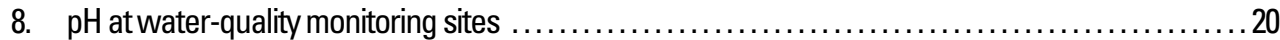

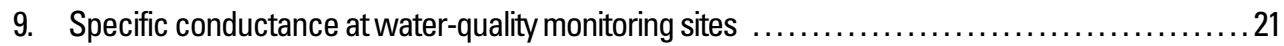

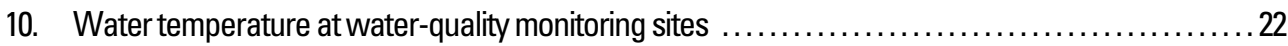

\section{Tables}

1. Locations of water-quality monitoring sites on the North Fork

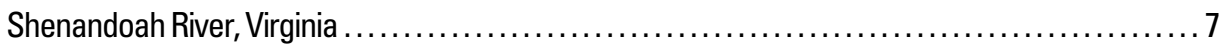

2. Water-quality sampling instrument specifications used in the water-quality

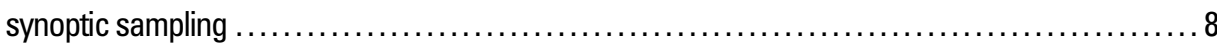

3. Minimum and maximum dissolved-oxygen concentrations and associated

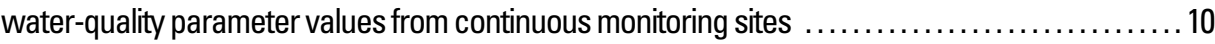

4. Early morning and late afternoon dissolved-oxygen concentrations and associated water-quality parameter values from instantaneous monitoring sites 


\section{Conversion Factors, Datum, and Abbreviated Water-Quality Units}

\begin{tabular}{|c|c|c|}
\hline Multiply & By & To obtain \\
\hline \multicolumn{3}{|c|}{ Length } \\
\hline inch (in.) & 2.54 & centimeter $(\mathrm{cm})$ \\
\hline inch (in.) & 25.4 & millimeter $(\mathrm{mm})$ \\
\hline foot $(\mathrm{ft})$ & 0.3048 & meter $(\mathrm{m})$ \\
\hline mile (mi) & 1.609 & kilometer (km) \\
\hline \multicolumn{3}{|c|}{ Area } \\
\hline cubic foot $\left(\mathrm{ft}^{3}\right)$ & 0.028317 & cubic meter \\
\hline square mile $\left(\mathrm{mi}^{2}\right)$ & 259.0 & hectare (ha) \\
\hline square mile $\left(\mathrm{mi}^{2}\right)$ & 2.590 & square kilometer $\left(\mathrm{km}^{2}\right)$ \\
\hline \multicolumn{3}{|c|}{ Volume } \\
\hline gallon (gal) & 3.785 & liter $(\mathrm{L})$ \\
\hline \multicolumn{3}{|c|}{ Mass } \\
\hline ounce, avoirdupois (oz) & 2.835 & milligram \\
\hline \multicolumn{3}{|c|}{ Flow } \\
\hline cubic foot per second $\left(\mathrm{ft}^{3} / \mathrm{s}\right)$ & 0.02832 & cubic meter per second \\
\hline
\end{tabular}

Temperature in degrees Celsius $\left({ }^{\circ} \mathrm{C}\right)$ may be converted to degrees Fahrenheit $\left({ }^{\circ} \mathrm{F}\right)$ as follows:

${ }^{\circ} \mathrm{F}=\left(1.8 \mathrm{x}^{\circ} \mathrm{C}\right)+32$

Vertical coordinate information is referenced to National Geodetic Vertical Datum of 1929 (NGVD 29)

Horizontal coordinate information is referenced to North American Datum of 1927 (NAD 27)

Abbreviated water-quality units: Chemical concentration is reported in milligrams per liter (mg/L). Milligrams per liter is a unit expressing the concentration of chemical constituents in solution as mass (milligrams) of solute per unit volume (liter) of water. For concentrations less than 7,000 $\mathrm{mg} / \mathrm{L}$, the numerical value is the same as for concentrations in parts per million. Specific electrical conductance of water is reported in microsiemens per centimeter at 25 degrees Celsius $(\mu \mathrm{S} / \mathrm{cm})$. 


\title{
Water-Quality Synoptic Sampling, July 1999: North Fork Shenandoah River, Virginia
}

\author{
By Jennifer L. Krstolic and Donald C. Hayes
}

\section{Abstract}

A study was conducted of water-quality conditions that may affect aquatic life during periods of low streamflow on the North Fork Shenandoah River, Va. Monthly mean streamflows in July 1999 at three streamflow-gaging stations were the lowest measured during the historical record on the river. Daily extremes of dissolved-oxygen concentrations were measured, along with $\mathrm{pH}$, specific conductance, and water- temperature values, at 52 sites along $80 \mathrm{mi}$ of the North Fork Shenandoah River from Cootes Store, Va., to its confluence with Passage Creek, near Strasburg, Va.

Dissolved-oxygen concentrations ranged from 2.1 to 16.4 milligrams per liter (mg/L). Dissolved-oxygen concentrations were equal to or less than the State water-quality minimum of $4.0 \mathrm{mg} / \mathrm{L}$ at 18 of 52 monitoring sites; all 18 sites were in the upper and middle portions of the river, where more than half of the first 34 sites had minimum dissolved-oxygen concentrations equal to or less than $4.0 \mathrm{mg} / \mathrm{L}$. There were large variations from minimum to maximum dissolved-oxygen concentrations, with concentrations fluctuating as much as $10 \mathrm{mg} / \mathrm{L}$ per day; and typically $5 \mathrm{mg} / \mathrm{L}$ per day during the study period.

$\mathrm{pH}$ ranged from 7.6 to 9.6 , with $\mathrm{pH}$ values frequently greater than 9.0 in the downstream portion of the river. Specificconductance values ranged from 178 to 856 microsiemens per centimeter $(\mu \mathrm{S} / \mathrm{cm})$, with values greater than $600 \mu \mathrm{S} / \mathrm{cm}$ only measured at a group of five sites in the upstream portion of the river. Air temperatures ranged from 21.0 to 37.0 degrees Celsius $\left({ }^{\circ} \mathrm{C}\right)$, and water temperatures ranged from 17.00 to $30.14^{\circ} \mathrm{C}$. Along the length of the North Fork Shenandoah River, longitudinal variation in water-quality parameters was small. Groups of sites that differed from the general pattern define reaches where increased monitoring may help determine the factors that affect water quality at those sites.

\section{Introduction}

The demands on the water resources of the Shenandoah River Basin in Virginia are a concern to a number of local, State, and Federal agencies, as well as private citizen groups. The demand on water resources has increased as a result of population growth in the past 20 years. From 1980 to 2000, popu- lation in the Shenandoah River Basin increased 30 percent, and water withdrawals for public supply and domestic use increased 40 percent (Solley and others, 1988, 1993, 1998; Hutson and others, 2004). Droughts in this region tax ground-water and surface-water supplies, which are used for public supply, domestic, and agricultural uses in the basin. In 1997, 1998, and 1999, drought conditions in the Shenandoah River Basin contributed to critical losses suffered by farmers, such as major damage to corn, hay, pasture, and small grain, as well as cattle weight loss. In response to these losses, Clarke County declared a state of emergency for three consecutive summers (Alison Teetor, Natural Resource Planner, Clarke County, Va., oral commun., 2004). During the 1999 low-flow period, mean monthly flows in July recorded at three streamflow-gaging stations on the North Fork Shenandoah River, a major tributary to the Shenandoah River, were the lowest observed over the 55- to 74-year periods of record (White and others, 2000). These droughts increased public awareness of the importance of a sustainable water supply and indicated a need for information on the availability and quality of water resources in the Shenandoah Basin.

There are concerns that streamflows are adequate not only for a sustainable public water supply but also to support the aquatic and terrestrial community, to support recreation, and to maintain aesthetic characteristics of rivers in the basin. The North Fork Shenandoah River Basin, which is located in Virginia and West Virginia, $75 \mathrm{mi}$ west of Washington, D.C. (fig. 1), supports a diverse aquatic and terrestrial community including 10 Federal or State listed species (indicating endangered, threatened, or special concern): freshwater mussels (brook floater, green floater, yellow lampmussel), isopods (Madison cave isopod, Shenandoah Valley cave isopod), reptiles and amphibians (wood turtle, cow knob salamander), birds (peregrine falcon), and bats (Indiana bat, Virginia big-eared bat) (Roble, 2001). Ensuring adequate streamflows to support these species and the diverse fish community (at least 37 species) (Persinger, 2003) not only protects the aquatic and terrestrial community in the North Fork Shenandoah Basin, but also maintains the river as a source for recreation.

In 1998, the U.S. Geological Survey (USGS), in cooperation with the Northern Shenandoah Valley Regional Commission (NSVRC) and Virginia Polytechnic Institute and State University (VPI), began a study to collect data and information on hydrology, habitat requirements of fish, and water use as part of a comprehensive assessment of water availability in the 


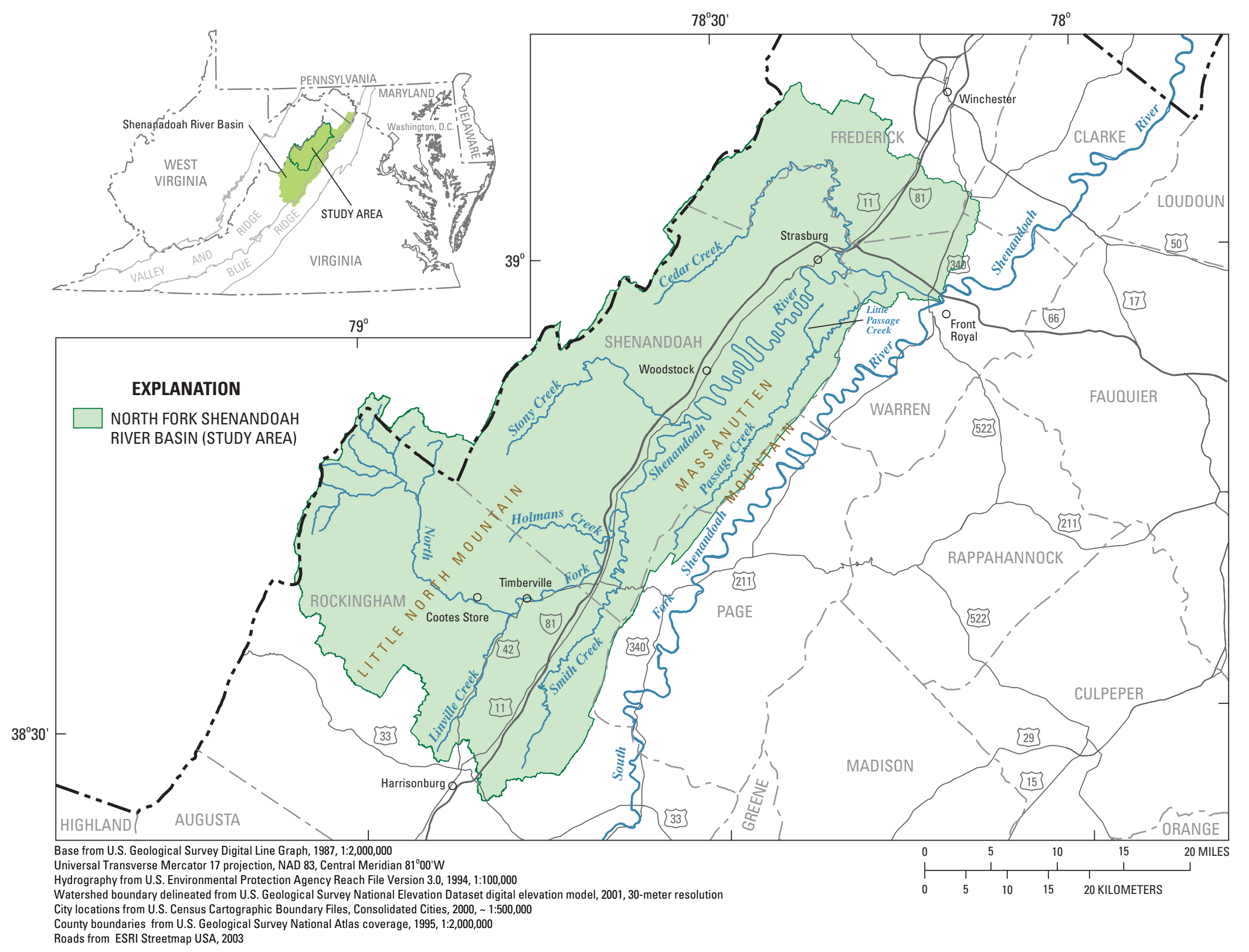

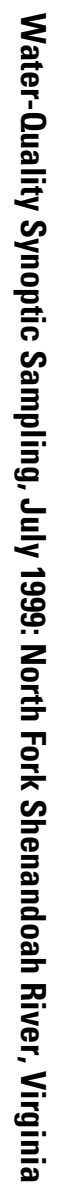

Figure 1. Location of the North Fork Shenandoah River Basin, Virginia (study area). 
North Fork Shenandoah River Basin. Prior to the onset of this study, Zappia and Hayes (1998) related water availability to the physical habitat needs of fish and other water uses along the Shenandoah River using Instream Flow Incremental Methodology (IFIM) techniques. Other studies have shown the utility of the IFIM process for water-resources management during lowflow conditions (Stalnaker, 1981; Leonard, Orth, and Goudreau, 1986; Orth and Leonard, 1990; Vadas and Weigmann, 1993). Following Zappia and Hayes (1998), IFIM techniques are being used in the North Fork Shenandoah River Basin to enhance understanding of low-flow conditions, to relate water availability to physical habitat needs of fish, and to provide science-based information for water management.

As part of the North Fork Shenandoah River IFIM study, water-quality data were collected in July 1999 at 52 sites along $80 \mathrm{mi}$ of the North Fork Shenandoah River from Cootes Store, Va., to the confluence with Passage Creek, near Strasburg, Va. The objective of this water-quality study was to measure daily minimum and maximum values of dissolved-oxygen concentrations, and to measure $\mathrm{pH}$, specific conductance, and water temperatures during low-flow conditions. Monitoring the variability of dissolved-oxygen concentrations throughout the river is important because a wide, fluctuating range of dissolved-oxygen concentrations may affect metabolism, growth, and development of fish (Wilding, 1939; Stewart and others, 1967; Whitworth, 1968; Fry, 1971; Davis, 1975).

\section{Purpose and Scope}

This report describes the daily fluctuations of dissolvedoxygen concentrations and reports associated water temperature, $\mathrm{pH}$, and specific-conductance values at 52 sites along 80 mi of the North Fork Shenandoah River from Cootes Store, Va., to its confluence with Passage Creek, near Strasburg, Va. Water-quality data were collected at 18 continuous monitoring sites and at 34 instantaneous monitoring sites from July 12-30, 1999.

This study was a short-term (less than one month) synoptic survey of water quality to measure daily minimum and maximum values of dissolved-oxygen concentrations and to contribute to the North Fork Shenandoah River IFIM study. This data-collection effort provides information about water-quality conditions that may affect aquatic life during summer low-flow periods (defined as flows less than the 90-percent exceedance flows) on the North Fork Shenandoah River, Va. The geographic distribution of water-quality values is presented in tables and on maps that show the range of values at each monitoring site relative to the site's location along the river.

\section{Description of Study Area}

The headwater streams that feed the North Fork Shenandoah River have their source in West Virginia, but the mainstem begins in Rockingham County, Va., and continues through Shenandoah and Warren Counties in Virginia. The river gener- ally flows northeast parallel to Interstate-81 and Highway-11 from Timberville, Va., to Front Royal, Va. The study sites included in this report are on an 80-mi-long portion of the North Fork Shenandoah River between Cootes Store, Va., and its confluence with Passage Creek near Strasburg, Va. (fig. 2), integrating a drainage area of $768 \mathrm{mi}^{2}$.

The North Fork Shenandoah River lies within the Valley and Ridge Physiographic Province. The basin topography is characterized by rolling hills and valleys, and is bordered on the eastern edge by Massanutten Mountain, which separates the North Fork Shenandoah River from the South Fork Shenandoah River (Virginia Department of Conservation and Economic Development, 1968). The northeast-southwest trending ridges of the Valley and Ridge are formed by resistant quartzite, sandstone, and conglomerates; the valleys are underlain by more readily weathered limestone, shale, and dolomite (Hack, 1965; Hayes, 1991). Karst topography over the limestone and dolomite rocks results in sinkholes, springs, and concentrated ground-water discharge to the river. The river has cut down to erosion-resistant bedrock along much of its length, giving it a characteristic shallow and wide channel. In the upper reaches, the North Fork Shenandoah River crosses the valleys and ridges around Little North Mountain, and then flows through the eastern side of the valley near Massanutten Mountain, where it has wide, gradual meanders (approximately 1.3-5.0 mi. in length between meanders) until it reaches Edinburg, Va. Downstream of Edinburg, Va., the river enters the Seven Bends area (fig. 2) where it is characterized by extremely narrow meanders (approximately $0.3 \mathrm{mi}$. in length between meanders). In the Seven Bends area there are 180-degree reversals, as the river winds in and out of the ridges on the eastern side of the valley. In this area, the river follows fracture zones in the shales of the Martinsburg Formation (Hack, 1965) that helped to form the narrow meanders which are common in the Seven Bends area (fig. 2).

Geographic location and topography strongly affect climate in the North Fork Shenandoah River Basin and in the Shenandoah River Basin overall. As frontal systems pass through the Valley and Ridge and Blue Ridge Physiographic Provinces of western Virginia (fig. 1), airflow is generally from the west. With westerly airflow, the Shenandoah River Basin is in the rain shadow of the mountains on the western edge of the Valley and Ridge; with easterly airflow, the basin is in the rain shadow of the Blue Ridge Mountains. As a result, the Shenandoah River Basin is one of the driest portions of the State, with a total annual rainfall typically a sparse $33.0 \mathrm{in}$. (Hayden and Michaels, 2003). During June, July, and August, mean rainfall is $11.0 \mathrm{in}$., with an average temperature range from 21.0 to $24.0^{\circ} \mathrm{C}$, and average maximum temperature range from 28.2 to $31.0^{\circ} \mathrm{C}$ (Southeast Regional Climate Center, 2003).

The Shenandoah River Basin has a rural character, with land use dominated by forest and wetlands (59 percent) and agriculture (38 percent) (U.S. Environmental Protection Agency, 1996). The basin population was 402,500 people in 2000, and has grown 17 percent from 345,100 people since the 1990 census (U.S. Census Bureau, 2002). Historically, the 


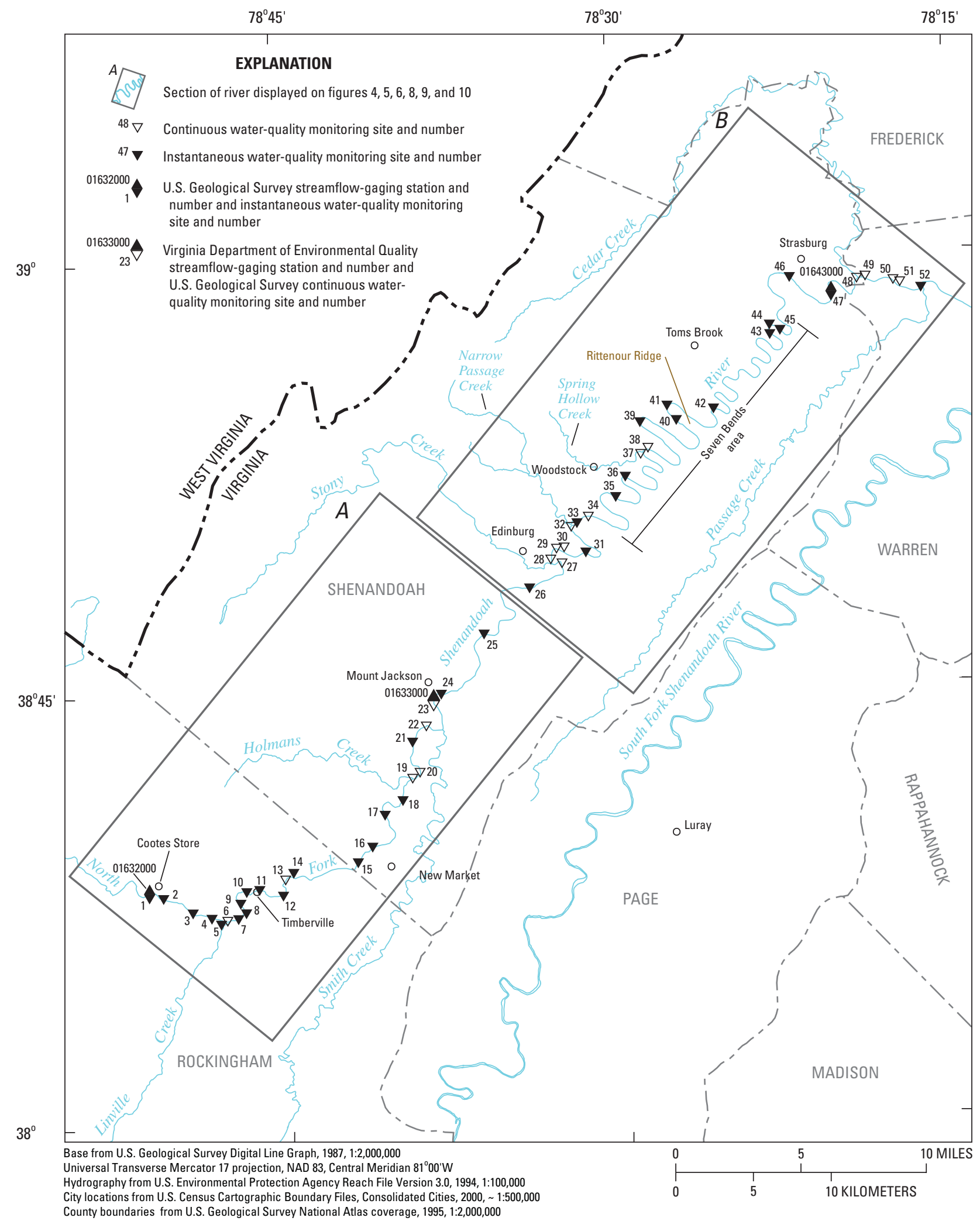

Figure 2. Locations of continuous and instantaneous water-quality monitoring sites, and streamflow-gaging stations on the North Fork Shenandoah River, Virginia. Site numbers increase downstream. 
economy of the area has been closely tied to rivers for agriculture, transportation, subsistence, and recreation. Currently (2004), economic activities in the basin are largely associated with livestock production (poultry and dairy cattle), agricultural-related industries, tourism and recreation, beverage bottling, and industries catering to universities and colleges. The rivers are both a water resource as well as a source of beauty and pride throughout the basin.

\section{Acknowledgments}

The authors thank the following for their expertise, time, and other support in completing the water quality portion of the North Fork Shenandoah River IFIM study: Dr. Donald Orth, VPI; the staff of the Department of Fisheries and Wildlife Sciences, VPI; and the members of the Northern Shenandoah Valley Regional Commission. The authors thank all the members of the Minimum Instream Flow technical advisory committee of NSVRC for their time and support, as well as their genuine interest in the health of the Shenandoah River Basin. The authors also thank Pat Maier and the Friends of the North Fork Shenandoah River for their assistance in contacting private land owners and obtaining permission for access to their land. The willingness of property owners to allow access to the river greatly assisted the data-collection efforts.

\section{Methods of Study}

As a part of the IFIM study along the North Fork Shenandoah River, water-quality data were collected during July of 1999 when the monthly mean streamflows were the lowest for the 74-year period of record at both the Cootes Store and Strasburg streamflow-gaging stations, and for the 55-year period of record for the Mount Jackson streamflow-gaging station (White and others, 2000). Discharge (streamflow) measurements were not made in conjunction with the synoptic sampling; however, the three gages recorded streamflows equivalent to the 98 - and 99-percent exceedance values, and were used as an indication of low streamflow conditions during the study period. Exceedance values are derived from a flow-duration curve, which is a cumulative frequency curve that shows the percent of time specified flows were equaled or exceeded during a given period (Searcy, 1959). Daily mean streamflow values ranging between the 25 and 75-percent exceedance values describe normal flow conditions.

During the study period (July 12-30, 2004), the USGS streamflow-gaging station at Cootes Store, Va., recorded daily mean streamflow values ranging from 0.55 to $1.2 \mathrm{ft}^{3} / \mathrm{s}$ (White and others, 2000) (fig. 3). These streamflows represent approximately the 99-percent exceedance value for the Cootes Store gage data during July and August. Based on the previous 50 years of data, typical flows during July and August at the Cootes Store gage range from $4.9 \mathrm{ft}^{3} / \mathrm{s}$ (75-percent exceedance value) to $40 \mathrm{ft}^{3} / \mathrm{s}$ (25-percent exceedance value).
During the study period, the Virginia Department of Environmental Quality (VDEQ) streamflow-gaging station near Mount Jackson, Va., recorded daily mean streamflow values ranging from $4.5 \mathrm{ft}^{3} / \mathrm{s}$ to $15.0 \mathrm{ft}^{3} / \mathrm{s}$ (White and others, 2000) (fig. 3). These streamflows are within the 98- and 99-percent exceedance values for the Mount Jackson gage data during July and August. Based on the previous 50 years of data, typical flows during July and August at the Mount Jackson gage range from $55 \mathrm{ft}^{3} / \mathrm{s}$ (75-percent exceedance value) to $159 \mathrm{ft}^{3} / \mathrm{s}$ (25-percent exceedance value).

During the study period, the USGS streamflow-gaging station near Strasburg, Va., recorded daily mean streamflow values ranging from $50 \mathrm{ft}^{3} / \mathrm{s}$ to $76 \mathrm{ft}^{3} / \mathrm{s}$ (White and others, 2000) (fig. 3). These streamflows represent approximately the 99-percent exceedance value for the Strasburg gage data during July and August. Based on the previous 50 years of data, typical flows during July and August at the Strasburg gage range from $122 \mathrm{ft}^{3} / \mathrm{s}$ (75-percent exceedance value) to $287 \mathrm{ft}^{3} / \mathrm{s}$ (25-percent exceedance value).

Low-flow conditions in the summer of 1999 were affected by lower than normal precipitation during the previous 12 months. From July 1998 to June 1999, the total monthly precipitation was 62 percent lower than normal, and on average 1.7$2.5 \mathrm{in}$. below the historical mean for each month except for January 1999 and March 1999 (Southeast Regional Climate Center, 2003). Precipitation during the fall of 1998 (September, October, and November) was 70 percent lower than the historical mean (Southeast Regional Climate Center, 2003). The reduced precipitation during the previous 12 months likely contributed to a decrease in both ground-water storage and groundwater discharge to the river.

Some precipitation fell during the field data collection period. In the downstream section of the basin, a total of $2.5 \mathrm{in}$. of rain was recorded at the weather station in Winchester, Va., during the study period: July 13 (0.1 in.), July 22 (0.6 in.), and July 29 (1.5 in.) (Southeast Regional Climate Center, 2003). The rain would have affected primarily the Cedar Creek drainage, and would have increased streamflow to the four study sites downstream of the confluence of Cedar Creek and the North Fork Shenandoah River. In the middle of the basin, a total of $1.2 \mathrm{in}$. of rain was recorded at the weather station in Woodstock, Va., during the study period: July 13 (0.1 in.), July 22 (0.8 in.), and July 29 (0.3 in.) (Southeast Regional Climate Center, 2003).

\section{Site Selection}

Monitoring sites for continuous water-quality data collection were selected on the basis of water-use information from VDEQ on permitted withdrawals and discharges within the basin (Virginia Department of Environmental Quality, written commun., 1998). The total permitted withdrawals or discharges were determined for six tributaries to the North Fork Shenandoah River by combining values of all permits within the tributary basin. Nine locations were selected for continuous 


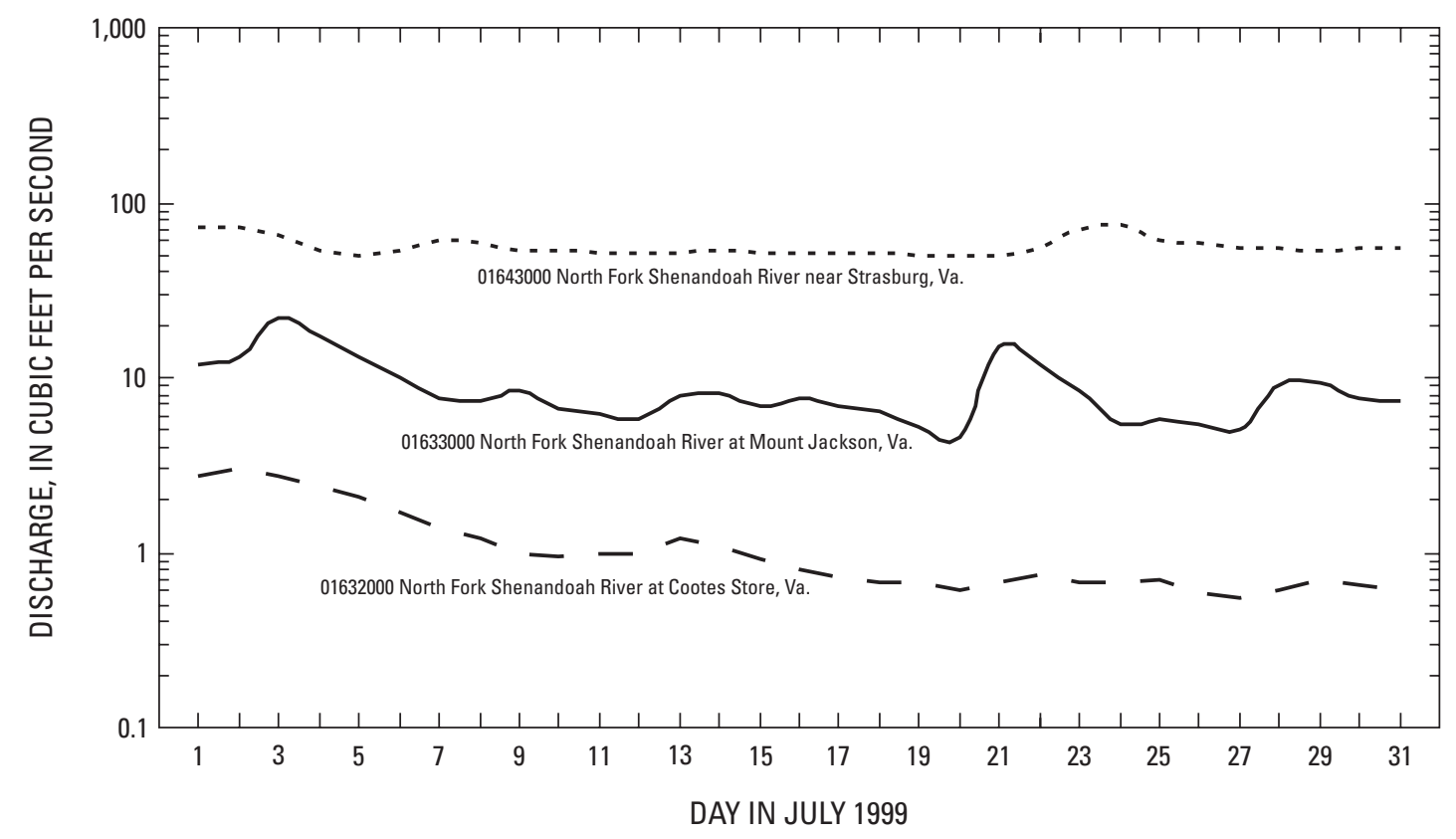

Figure 3. Streamflow in July 1999 for 3 streamflow-gaging stations on the North Fork Shenandoah River, Virginia.

water-quality data collection at the confluence of tributaries with substantial total permitted withdrawals or discharges, and along the mainstem where permitted withdrawal or discharge values were large. During the data-collection effort, monitoring instruments were placed upstream and downstream of each of the 9 locations for a total of 18 continuous water-quality monitoring sites.

In addition to the continuous water-quality monitoring sites, 34 monitoring sites were selected for instantaneous water-quality data collection. It was desirable to sample as many sites on the river as possible in order to document longitudinal variations in water quality. Geographic constraints limited the number of sites available for instantaneous water-quality measurements; however, bridges, boat ramps, local parks, and some private property served as access points for the instantaneous water-quality monitoring sites. All available access points were included in the study.

Location information for continuous and instantaneous water-quality monitoring sites is in table 1 and figure 2 . Site numbers increase in the downstream direction, with site 1 being the most upstream site and site 52 the most downstream site.

\section{Instrumentation}

All instruments used for collection of water-quality field parameters were of the YSI-6 series, and contain sensors with identical specifications (table 2). Instruments used for continuous data collection were YSI 600XLM and YSI 6920 models; instruments used for instantaneous data collection were YSI 600XL models. The instruments contain sensors for measuring dissolved oxygen, $\mathrm{pH}$, specific conductance, and temperature.
All instruments were checked for temperature accuracy in the office before the field data collection began, and again after field data collection was completed. Water temperature was accurate within $0.5^{\circ} \mathrm{C}$ of a calibrated analog thermometer at near-freezing temperature and at room temperature.

Water-quality monitoring instruments used for continuous monitoring were calibrated for dissolved oxygen and $\mathrm{pH}$, and compared against standards for specific conductance immediately prior to field deployment. Monitors used for instantaneous sampling were calibrated in the same manner prior to field deployment, and calibrated twice daily during field data collection.

\section{Water-Quality Field Parameters}

The water-quality parameters measured at both continuous and instantaneous monitoring sites were dissolved oxygen, $\mathrm{pH}$, specific conductance, and water temperature. Additionally, air temperature and barometric pressure were measured at instantaneous monitoring sites. Study methods were designed so that the associated parameters were measured concurrently with the expected maximum and minimum dissolved-oxygen concentrations for each site; associated parameter values, therefore, do not necessarily represent maximum and minimum values. USGS protocols were followed for sampling-equipment cleaning, calibration, and data collection (U.S. Geological Survey, 1997). 
Table 1. Locations of water-quality monitoring sites on the North Fork Shenandoah River, Virginia.

[Bold text indicates continuous water-quality monitoring sites; plain text indicates instantaneous monitoring sites; DMS.S, degrees minutes decimal-seconds; NAD27, North American datum of 1927]

\begin{tabular}{|c|c|c|c|}
\hline $\begin{array}{l}\text { Site number } \\
\text { (Fig. 2) }\end{array}$ & Site name & $\begin{array}{c}\text { Latitude } \\
\text { DMS.S (NAD27) }\end{array}$ & $\begin{array}{c}\text { Longitude } \\
\text { DMS.S (NAD27) }\end{array}$ \\
\hline 1 & At Cootes Store, Va. & N 383813.6 & W 0785110.1 \\
\hline 2 & At Evans Ford near Cootes Store, Va. & N 383759.8 & W 0785032.3 \\
\hline 3 & Along Route 789 near Cootes Store, Va. & N 383728.0 & W 0784915.2 \\
\hline 4 & At Route 617 at Broadway, Va. & N 383715.9 & W 0784807.1 \\
\hline 5 & At Route 411 at Broadway, Va. & N 383710.9 & W 0784743.9 \\
\hline 6 & At Route 411 at Broadway, Va. & N 383711.0 & W 0784743.9 \\
\hline 7 & Below Linville Creek at Broadway, Va. & N 383714.6 & W 0784728.3 \\
\hline 8 & At dam at Timberville, Va. & N 383738.2 & W 0784659.5 \\
\hline 9 & Below dam at Timberville, Va. & N 383740.5 & W 0784702.5 \\
\hline 10 & At Memorial Park at Timberville, Va. & N 383810.0 & W 0784651.3 \\
\hline 11 & Route 42 at Timberville, Va. & N 383815.2 & W 0784625.8 \\
\hline 12 & Above Shenville farm at Timberville, Va. & N 383820.3 & W 0784511.4 \\
\hline 13 & Below Shenville farm at Timberville, Va. & N 383833.0 & W 0784508.4 \\
\hline 14 & Below Shenville farm at Timberville, Va. & N 383834.7 & W 0784507.9 \\
\hline 15 & At Route 617 at New Market, Va. & N 383906.5 & W 0784152.9 \\
\hline 16 & Along Route 617 at New Market, Va. & N 383938.3 & W 0784113.6 \\
\hline 17 & Along Route 736 at Quicksburg, Va. & N 384044.1 & W 0784038.4 \\
\hline 18 & Along Route 767 at Quicksburg, Va. & N 384113.5 & W 0783950.2 \\
\hline 19 & Above Holmans Creek near Quicksburg, Va. & N 384159.2 & W 0783923.4 \\
\hline 20 & Below Holmans Creek near Quicksburg, Va. & N 384159.9 & W 0783920.8 \\
\hline 21 & At Route 720 near Mount Jackson, Va. & N 384314.9 & W 0783920.5 \\
\hline 22 & At Route 11 at Mount Jackson, Va. & N 384349.0 & W 0783843.2 \\
\hline 23 & At Mount Jackson, Va. & N 384444.3 & W 0783821.7 \\
\hline 24 & At Mount Jackson, Va. & N 384445.2 & W 0783820.6 \\
\hline 25 & At Route 707 near Mount Jackson, Va. & N 384656.3 & W 0783602.9 \\
\hline 26 & At Route 698 at Edinburg, Va. & N 384830.2 & W 0783358.4 \\
\hline 27 & Above Stoney Creek at Edinburg, Va. & N 384920.0 & W 0783254.6 \\
\hline 28 & Below Stoney Creek at Edinburg, Va. & N 384927.4 & W 0783259.9 \\
\hline 29 & Above dam at Edinburg, Va. & N 384951.4 & W 0783242.9 \\
\hline 30 & Below dam at Edinburg, Va. & N 384949.8 & W 0783240.5 \\
\hline 31 & Along Route 673 near Edinburg, Va. & N 384941.7 & W 0783125.8 \\
\hline 32 & Above Narrow Passage Creek at Route 672 & N 385041.1 & W 0783155.7 \\
\hline 33 & Near boat ramp at Narrow Passage Creek & N 385043.4 & W 0783147.6 \\
\hline 34 & Below Narrow Passage Creek near Edinburg Va. & N 385047.7 & W 0783142.3 \\
\hline 35 & At Route 609 near Woodstock, Va. & N 385135.6 & W 0783002.7 \\
\hline 36 & At Lupton Road at Woodstock, Va. & N 385217.4 & W 0782935.3 \\
\hline 37 & Above Spring Hollow Creek at Woodstock, Va. & N 385306.9 & W 0782848.0 \\
\hline 38 & Below Spring Hollow Creek at Woodstock, Va. & N 385312.7 & W 0782845.5 \\
\hline 39 & At Route 663 at Woodstock, Va. & N 385410.2 & W 0782852.5 \\
\hline 40 & Along Rittenour Ridge near Woodstock, Va. & N 385412.4 & W 0782715.2 \\
\hline 41 & At Route 661 near Woodstock, Va. & N 385443.1 & W 0782739.1 \\
\hline 42 & At Route 654 near Maurertown, Va. & N 385434.7 & W 0782535.3 \\
\hline 43 & Along Route 648 near Fishers Hill, Va. & N 385706.3 & W 0782259.5 \\
\hline 44 & Along Route 644 near Fishers Hill, Va. & N 385725.9 & W 0782300.2 \\
\hline
\end{tabular}


Table 1. Continued.

\begin{tabular}{clcc}
\hline $\begin{array}{c}\text { Site number } \\
\text { (Fig. 2) }\end{array}$ & \multicolumn{1}{c}{ Site name } & $\begin{array}{c}\text { Latitude } \\
\text { DMS.S (NAD27) }\end{array}$ & $\begin{array}{c}\text { Longitude } \\
\text { DMS.S (NAD27) }\end{array}$ \\
\hline 45 & At Route 744 near Fishers Hill, Va. & N 385714.6 & W 0782232.1 \\
46 & At Route 648 at Strasburg, Va. & N 385903.9 & W 0782203.1 \\
47 & Near Strasburg, Va. & N 385837.4 & W 0782011.5 \\
$\mathbf{4 8}$ & Above Cedar Creek near Strasburg, Va. & $\mathbf{N ~ 3 8 5 8 5 8 . 2}$ & W 0781904.0 \\
$\mathbf{4 9}$ & Below Cedar Creek near Strasburg, Va. & $\mathbf{N ~ 3 8 5 9 0 0 . 8}$ & W 0781857.1 \\
$\mathbf{5 0}$ & Above Winchester Dam at Waterlick, Va. & $\mathbf{N ~ 3 8 5 8 5 2 . 7}$ & W 0781726.1 \\
$\mathbf{5 1}$ & Below Winchester Dam at Waterlick, Va. & $\mathbf{N ~ 3 8 5 8 4 9 . 4}$ & W 0781723.7 \\
52 & Above Passage Creek at Waterlick, Va. & N 385835.6 & W 0781613.3 \\
\hline
\end{tabular}

Table 2. Water-quality sampling instrument specifications used in the water-quality synoptic sampling on the North Fork Shenandoah River, Virginia.

[ ${ }^{\circ} \mathrm{C}$, degrees Celsius; $\mathrm{mS} / \mathrm{cm}$, millisiemens per centimeter; \%, percent; air sat, air saturation; $\mathrm{mg} / \mathrm{L}$, milligrams per liter]

\begin{tabular}{lll}
\hline \multicolumn{1}{c}{$\begin{array}{c}\text { Water-quality } \\
\text { field parameter }\end{array}$} & \multicolumn{1}{c}{ Range $^{1}$} & \multicolumn{1}{c}{ Accuracy $^{1}$} \\
\hline Temperature & $-5-45^{\circ} \mathrm{C}$ & $\pm 0.15^{\circ} \mathrm{C}$ \\
Specific conductance & $0-100 \mathrm{mS} / \mathrm{cm}$ & $\pm 0.5 \%$ reading $+0.001 \mathrm{mS} / \mathrm{cm}$ \\
$\mathrm{pH}$ & $0-14$ units & $\pm 0.2 \mathrm{units}$ \\
Dissolved oxygen, percent air & $0-500 \%$ air sat & $0-200 \%$ air sat: $\pm 2 \%$ reading or $2 \%$ air sat (the greater value) \\
$\quad$ & & $200-500 \%$ air sat: $\pm 6 \%$ of reading \\
saturation & $0-50 \mathrm{mg} / \mathrm{L}$ & $0-20 \mathrm{mg} / \mathrm{L}: \pm 2 \%$ of reading or $0.2 \mathrm{mg} / \mathrm{L}$ (the greater value) \\
Dissolved oxygen, $\mathrm{mg} / \mathrm{L}$ & & $20-50 \mathrm{mg} / \mathrm{L}: \pm 6 \%$ of reading \\
\hline
\end{tabular}

\footnotetext{
${ }^{1}$ From YSI Incorporated, 1998, Environmental Monitoring Systems operations manual: 6-series, Revision A, Yellow Springs, Ohio, 255 p.
} 


\section{Continuous Water-Quality Field Measurements}

Self-contained water-quality monitors and data loggers were used to measure and record dissolved oxygen, $\mathrm{pH}$, specific conductance, and water temperature at 18 continuous monitoring sites. Most continuous monitoring sites were within $500 \mathrm{ft}$ of a permitted withdrawal or discharge; however, some continuous monitoring sites were located on either end of a stream reach with numerous permitted sites to assess water quality upstream and downstream of the reach. At each continuous water-quality monitoring site, a YSI 600XLM or YSI 6920 multi-parameter instrument was housed within a section of polyvinylchloride (PVC) pipe that was secured to the stream bottom. The water-quality monitor was placed in the centroid of flow, with the sensors elevated above the streambed and other obstructions to flow such as large boulders and logs. The average depth of all continuous monitoring sites was $2.3 \mathrm{ft}$, and the median depth was $1.7 \mathrm{ft}$. Because the water was shallow, elevating the sensors above the streambed and any obstructions placed them approximately in the flowing portion of the water column. At the few sites that were greater than $3 \mathrm{ft}$ deep, the water-quality monitor was placed $1-3 \mathrm{ft}$ below the water surface.

Water-quality data were collected semi-hourly (every 30 minutes, on the hour and half-hour) for 1-8 days during July 12-30, 1999. Maximum and minimum dissolved-oxygen concentrations were taken from the semi-hourly data for each of the 18 sites. These data represent the greatest (MAX) and least (MIN) semi-hourly dissolved-oxygen concentrations recorded during the study period, but are not necessarily values that were measured on the same day. Instead, these values are the upper and lower limits of the range of measured concentrations.

\section{Instantaneous Water-Quality Field Measurements}

Hand-held water-quality monitors were used to measure dissolved oxygen, $\mathrm{pH}$, specific conductance, and water temperature at 34 sites along the river. Air temperature and barometric pressure also were measured and recorded at these sites. Water-quality data were collected once in the early morning (AM) and once in the late afternoon (PM) for each site from July 27-29, 1999. The data were collected just before sunrise and just before sunset when the daily minimum and maximum dissolved-oxygen concentrations would be expected, respectively. The study design did not allow for depth-integrated sampling along a cross section at each site, but the sampling was done in a part of the channel that was representative of streamflow. Water-quality measurements were made at approximately 60 percent of water depth.

\section{Results of Water-Quality Synoptic Sampling}

Throughout this report, the order of the data is from site 1, near Cootes Store, Va., to site 52, the most downstream site, near the confluence of the North Fork Shenandoah River with Passage Creek. Maximum and minimum dissolved-oxygen concentrations and associated parameter values of $\mathrm{pH}$, specific conductance, and water temperature from the continuous and instantaneous water-quality monitoring sites are in tables 3 and 4. Maximum and minimum values for the associated parameters of $\mathrm{pH}$, specific conductance, and water temperature not found in table 3 may be in the appendix, which contains the complete set of semi-hourly data for continuous monitoring sites. The longitudinal distribution of water-quality values is displayed on a map of the North Fork Shenandoah River Basin (figs. 4, 5, 6, 8,9 , and 10). On these figures, the 52 study sites and associated water-quality data are divided into two sections, with sites 1-25 in section A and sites 26-52 in section B. These divisions are arbitrary and were made for displaying the data.

\section{Dissolved Oxygen}

There were large variations in MIN and MAX values of dissolved-oxygen concentrations recorded with continuous measurement techniques, as well as in AM and PM values recorded with instantaneous measurement techniques. Dissolved-oxygen concentrations from both continuous and instantaneous water-quality monitoring sites ranged from $2.1 \mathrm{mg} / \mathrm{L}$ to $16.4 \mathrm{mg} / \mathrm{L}$; dissolved oxygen as percent air saturation ranged from 25 to 204 percent (figs. 4 and 5, tables 3 and 4). The State numerical criterion for the minimum concentration of dissolved oxygen in Piedmont and Mountainous zones is $4.0 \mathrm{mg} / \mathrm{L}$ (State Water Control Board, 2002). State water-quality criteria are designed to maintain water-quality conditions conducive to healthy aquatic communities. Dissolved-oxygen concentrations were equal to or less than the State water-quality minimum at 18 of 52 monitoring sites; all 18 sites were in the upper and middle portions of the river, where more than half of the first 34 sites had minimum dissolved-oxygen concentrations equal to or less than $4.0 \mathrm{mg} / \mathrm{L}$ (fig. 4). No dissolved-oxygen concentrations less than the State minimum were recorded downstream of site 34 near Narrow Passage Creek (fig. 4).

The results from continuous sampling (MIN and MAX values) and instantaneous sampling (AM and PM values) were compared. To compare the values that approximate the daily minimum dissolved-oxygen concentration, a Wilcoxon twosample ranked sum test (Ott and Longnecker, 2001) of the MIN and AM datasets was completed. The mean of the MIN dataset was $4.4 \mathrm{mg} / \mathrm{L}$, and the median was $4.7 \mathrm{mg} / \mathrm{L}$; the mean of the AM dataset was $4.9 \mathrm{mg} / \mathrm{L}$, and the median was $4.7 \mathrm{mg} / \mathrm{L}$. Results from the Wilcoxon test showed no significant difference between the two datasets ( $\mathrm{p}$-value $=0.8099$ ). To compare the values that approximate the daily maximum dissolved-oxygen concentration, a Wilcoxon two-sample ranked sum test of the MAX and PM datasets was completed. The mean of the PM dataset was $11.0 \mathrm{mg} / \mathrm{L}$, and the median also was $11.0 \mathrm{mg} / \mathrm{L}$; the mean of the MAX dataset was $12.0 \mathrm{mg} / \mathrm{L}$, and the median was $12.9 \mathrm{mg} / \mathrm{L}$. Results from the Wilcoxon test showed no significant difference between the two datasets ( $p$-value $=0.4901$ ) 
Table 3. Minimum and maximum dissolved-oxygen concentrations and associated water-quality parameter values from continuous monitoring sites on the North Fork Shenandoah River, Virginia. [MIN, minimum dissolved-oxygen concentration; MAX, maximum dissolved-oxygen concentration; ${ }^{\circ} \mathrm{C}$, degrees Celsius; DOsat, dissolved oxygen as percent air saturation; DO, dissolved oxygen; $\mathrm{mg} / \mathrm{L}$, milligrams per liter; $\mathrm{SC}$, specific conductance; $\mu \mathrm{S} / \mathrm{cm}$, microsiemens per centimeter; -, no data]

\begin{tabular}{|c|c|c|c|c|c|c|c|c|c|c|c|c|c|c|}
\hline $\begin{array}{c}\text { Site } \\
\text { number } \\
\text { (Fig. 2) }\end{array}$ & $\begin{array}{c}\text { Begin } \\
\text { date }\end{array}$ & $\begin{array}{c}\text { Begin } \\
\text { time } \\
\text { (local) }\end{array}$ & $\begin{array}{l}\text { End } \\
\text { date }\end{array}$ & $\begin{array}{c}\text { End } \\
\text { time } \\
\text { (local) }\end{array}$ & $\begin{array}{c}\text { Water } \\
\text { depth } \\
\text { (ft) }\end{array}$ & Statistic & Date & $\begin{array}{c}\text { Time } \\
\text { (local) }\end{array}$ & $\begin{array}{c}\text { DOsat } \\
\text { (percent) }\end{array}$ & $\begin{array}{c}\mathrm{DO} \\
(\mathrm{mg} / \mathrm{L})\end{array}$ & $\begin{array}{c}\text { DO1 }^{1} \\
\text { (ratio) }\end{array}$ & $\begin{array}{c}\mathrm{pH} \\
\text { (standard } \\
\text { units) }\end{array}$ & $\begin{array}{c}\text { SC } \\
(\mu \mathrm{S} / \mathbf{c m})\end{array}$ & $\begin{array}{c}\text { Water } \\
\text { temperature } \\
\text { ('C) }\end{array}$ \\
\hline \multirow{2}{*}{6} & \multirow{2}{*}{ 07/29/1999 } & \multirow{2}{*}{$14: 30$} & \multirow{2}{*}{ 07/30/1999 } & \multirow{2}{*}{$10: 00$} & \multirow{2}{*}{1.5} & MIN & 07/30/1999 & $07: 30$ & 43.7 & 3.7 & \multirow{2}{*}{3.4} & 7.7 & 295 & 24.40 \\
\hline & & & & & & MAX & 07/29/1999 & 18:00 & 162.6 & 12.7 & & 8.5 & 316 & 28.12 \\
\hline \multirow{2}{*}{13} & \multirow{2}{*}{ 07/29/1999 } & \multirow{2}{*}{$12: 00$} & \multirow{2}{*}{ 07/30/1999 } & \multirow{2}{*}{$9: 30$} & \multirow{2}{*}{1.7} & MIN & 07/30/1999 & 03:00 & 32.9 & 2.7 & \multirow{2}{*}{6.1} & - & 800 & 24.75 \\
\hline & & & & & & MAX & 07/29/1999 & $14: 30$ & 203.5 & 16.4 & & - & 713 & 26.21 \\
\hline \multirow{2}{*}{19} & \multirow{2}{*}{ 07/15/1999 } & \multirow{2}{*}{$11: 30$} & \multirow{2}{*}{ 07/20/1999 } & \multirow{2}{*}{$12: 00$} & \multirow{2}{*}{2.0} & MIN & 07/20/1999 & 06:00 & 26.2 & 2.2 & \multirow{2}{*}{6.8} & 7.6 & 457 & 24.32 \\
\hline & & & & & & MAX & 07/18/1999 & $13: 30$ & 192.9 & 15.0 & & 8.8 & 429 & 28.27 \\
\hline \multirow{2}{*}{20} & \multirow{2}{*}{ 07/15/1999 } & \multirow{2}{*}{$12: 30$} & \multirow{2}{*}{ 07/20/1999 } & \multirow{2}{*}{$12: 00$} & \multirow{2}{*}{2.1} & MIN & 07/20/1999 & 06:00 & 36.8 & 3.1 & 40 & 7.7 & - & 24.29 \\
\hline & & & & & & MAX & 07/18/1999 & $13: 30$ & 198.0 & 15.3 & 4.9 & 8.9 & - & 28.73 \\
\hline 22 & $07 / 28 / 1999$ & $14: 30$ & 07/30/1999 & $8 \cdot 30$ & 28 & MIN & 07/29/1999 & 09:30 & 29.6 & 2.5 & & 7.6 & 446 & 24.16 \\
\hline 22 & $01 / 20 / 1999$ & 14.50 & & $8: 30$ & 2.8 & MAX & 07/28/1999 & $17: 30$ & 79.7 & 6.5 & 2.6 & 8.0 & 423 & 25.72 \\
\hline 23 & & 12.00 & $07 / 30 / 1000$ & 8.00 & 2 & MIN & 07/30/1999 & $06: 30$ & 50.5 & 4.3 & 24 & 7.8 & 432 & 23.88 \\
\hline 23 & $01 / 20 / 1999$ & 15.00 & $01 / 30 / 1999$ & 0.00 & 2.1 & MAX & 07/28/1999 & $15: 00$ & 127.8 & 10.3 & 2.4 & 8.4 & 442 & 26.48 \\
\hline 27 & $07 / 26 / 1999$ & $16 \cdot 30$ & $07 / 28 / 1999$ & $7 \cdot 30$ & 14 & MIN & 07/27/1999 & 08:00 & 57.7 & 5.0 & 32 & 7.7 & 390 & 22.68 \\
\hline & & & & $1: 30$ & & MAX & $07 / 27 / 1999$ & $17: 30$ & 198.5 & 15.9 & 3.2 & 8.6 & 366 & 26.83 \\
\hline 28 & $0726 / 1090$ & 16.00 & $07 / 28 / 1000$ & 7.30 & 15 & MIN & 07/27/1999 & $07: 30$ & 53.9 & 4.7 & 31 & 7.8 & 419 & 22.20 \\
\hline 28 & $0 / / 20 / 1999$ & $10: 00$ & $0 / 128 / 1999$ & $1: 30$ & 1.5 & MAX & 07/27/1999 & $17: 30$ & 178.6 & 14.4 & 3.1 & 8.7 & 391 & 25.29 \\
\hline 29 & $07 / 26 / 1999$ & $15 \cdot 00$ & $07 / 28 / 1999$ & 8.00 & 100 & MIN & 07/27/1999 & $17: 00$ & 68.1 & 5.7 & 23 & 7.9 & 449 & 23.93 \\
\hline 29 & $0 / / 20 / 1999$ & $15: 00$ & $0 / 128 / 1999$ & $8: 00$ & 10.0 & MAX & 07/27/1999 & $22: 00$ & 157.5 & 13.1 & 2.3 & 8.5 & 436 & 24.68 \\
\hline 30 & $07 / 26 / 1090$ & $14 \cdot 30$ & $07 / 28 / 1090$ & 8.00 & 22 & MIN & 07/27/1999 & $07: 30$ & 83.3 & 7.1 & & 8.0 & 438 & 23.23 \\
\hline 30 & $0 / 726 / 1999$ & $14: 30$ & $0 / 128 / 1999$ & $8: 00$ & 2.2 & MAX & 07/27/1999 & $15: 00$ & 105.4 & 9.0 & 1.3 & 8.1 & 438 & 23.17 \\
\hline 32 & $07 / 12 / 1099$ & $10 \cdot 00$ & $07 / 20 / 1999$ & $16 \cdot 00$ & 06 & MIN & 07/14/1999 & 07:30 & 36.7 & 3.2 & 45 & 8.3 & 471 & 22.14 \\
\hline 32 & $01 / 1271999$ & $19: 00$ & $0 / / 20 / 1999$ & 10.00 & 0.0 & MAX & 07/19/1999 & $17: 30$ & 181.6 & 14.4 & 4.5 & 8.8 & 451 & 27.11 \\
\hline 34 & $07 / 12 / 1999$ & $18 \cdot 30$ & $07 / 20 / 1999$ & $15 \cdot 00$ & 0.5 & MIN & 07/20/1999 & 08:00 & 57.6 & 4.8 & & 8.2 & 470 & 25.09 \\
\hline 34 & $01 / 12 / 1999$ & $18: 30$ & $0 / / 20 / 1999$ & $15: 00$ & 0.5 & MAX & 07/16/1999 & $17: 30$ & 168.0 & 13.4 & 2.8 & 8.8 & 455 & 26.72 \\
\hline 37 & 10001000 & 11.00 & & 0.00 & 20 & MIN & 07/26/1999 & 07:00 & 59.4 & 4.7 & 1 & 8.4 & 438 & 26.91 \\
\hline 31 & $01 / 21 / 1999$ & 11.00 & 0112171999 & 9.00 & 2.0 & MAX & 07/26/1999 & $18: 30$ & 132.9 & 10.0 & 2.1 & 8.8 & 425 & 30.12 \\
\hline 38 & $07 / 21 / 1999$ & $11 \cdot 30$ & $07 / 27 / 1999$ & $9 \cdot 30$ & 16 & MIN & 07/24/1999 & 04:00 & 62.4 & 4.9 & 19 & 8.4 & 431 & 27.51 \\
\hline 38 & $01 / 21 / 1999$ & $11: 30$ & 0112111999 & $9: 30$ & 1.0 & MAX & 07/26/1999 & $18: 00$ & 124.0 & 9.4 & 1.9 & 8.7 & 433 & 29.66 \\
\hline
\end{tabular}


Table 3. Continued.

\begin{tabular}{|c|c|c|c|c|c|c|c|c|c|c|c|c|c|c|}
\hline $\begin{array}{c}\text { Site } \\
\text { number } \\
\text { (Fig. 2) }\end{array}$ & $\begin{array}{l}\text { Begin } \\
\text { date }\end{array}$ & $\begin{array}{c}\text { Begin } \\
\text { time } \\
\text { (local) }\end{array}$ & $\begin{array}{l}\text { End } \\
\text { date }\end{array}$ & $\begin{array}{c}\text { End } \\
\text { time } \\
\text { (local) }\end{array}$ & $\begin{array}{c}\text { Water } \\
\text { depth } \\
\text { (ft) }\end{array}$ & Statistic & Date & $\begin{array}{c}\text { Time } \\
\text { (local) }\end{array}$ & $\begin{array}{c}\text { DOsat } \\
\text { (percent) }\end{array}$ & $\begin{array}{c}\mathrm{DO} \\
(\mathrm{mg} / \mathrm{L})\end{array}$ & $\begin{array}{c}\mathrm{DO}^{1} \\
\text { (ratio) }\end{array}$ & $\begin{array}{c}\mathrm{pH} \\
\text { (standard } \\
\text { units) }\end{array}$ & $\begin{array}{c}\text { SC } \\
(\mu \mathrm{S} / \mathrm{cm})\end{array}$ & $\begin{array}{c}\text { Water } \\
\text { temperature } \\
\text { ('C) }\end{array}$ \\
\hline \multirow{2}{*}{48} & \multirow{2}{*}{ 07/27/1999 } & \multirow{2}{*}{$15: 00$} & \multirow{2}{*}{ 07/29/1999 } & \multirow{2}{*}{$8: 30$} & \multirow{2}{*}{-} & MIN & $07 / 29 / 1999$ & $07: 30$ & 69.6 & 5.7 & \multirow{2}{*}{1.5} & 9.2 & 406 & 25.46 \\
\hline & & & & & & MAX & 07/28/1999 & $13: 00$ & 111.4 & 8.6 & & 9.3 & 410 & 28.93 \\
\hline \multirow{2}{*}{49} & \multirow{2}{*}{ 07/27/1999 } & \multirow{2}{*}{$15: 30$} & \multirow{2}{*}{ 07/29/1999 } & \multirow{2}{*}{$8: 30$} & \multirow{2}{*}{1.6} & MIN & 07/29/1999 & $07: 30$ & 71.9 & 5.9 & \multirow{2}{*}{1.5} & 9.1 & 395 & 24.96 \\
\hline & & & & & & MAX & 07/28/1999 & $13: 00$ & 113.1 & 8.8 & & 9.2 & 397 & 28.38 \\
\hline \multirow{2}{*}{50} & \multirow{2}{*}{ 07/28/1999 } & \multirow{2}{*}{$14: 00$} & \multirow{2}{*}{ 07/30/1999 } & \multirow{2}{*}{$8: 30$} & \multirow{2}{*}{4.4} & MIN & 07/29/1999 & 10:00 & 66.8 & 5.5 & \multirow{2}{*}{1.8} & 8.9 & 403 & 25.39 \\
\hline & & & & & & MAX & 07/29/1999 & $21: 30$ & 123.0 & 9.7 & & 9.1 & 390 & 27.81 \\
\hline \multirow{2}{*}{51} & \multirow{2}{*}{ 07/29/1999 } & \multirow{2}{*}{$9: 30$} & \multirow{2}{*}{ 07/30/1999 } & \multirow{2}{*}{ 9:00 } & \multirow{2}{*}{1.5} & MIN & 07/29/1999 & $10: 00$ & 77.9 & 6.4 & \multirow{2}{*}{1.4} & 8.9 & 402 & 25.46 \\
\hline & & & & & & MAX & 07/29/1999 & $22: 30$ & 113.5 & 8.9 & & 9.1 & 392 & 27.73 \\
\hline
\end{tabular}

${ }^{1}$ Calculated by dividing the maximum dissolved-oxygen concentration by the minimum dissolved-oxygen concentration 
Table 4. Early morning and late afternoon dissolved-oxygen concentrations and associated water-quality parameter values from instantaneous monitoring sites on the North Fork Shenandoah River, Virginia.

[AM, early morning dissolved-oxygen concentration; PM, late afternoon dissolved-oxygen concentration; ${ }^{\circ} \mathrm{C}$, degrees Celsius; DOsat, dissolved oxygen as percent air saturation; DO, dissolved oxygen; mg/L, milligrams per liter; $\mathrm{mm} \mathrm{Hg}$, millimeters of mercury; $\mathrm{SC}$, specific conductance; $\mu \mathrm{S} / \mathrm{cm}$, microsiemens per centimeter; -, no data]

\begin{tabular}{|c|c|c|c|c|c|c|c|c|c|c|c|}
\hline $\begin{array}{c}\text { Site } \\
\text { number } \\
\text { (Fig. 2) }\end{array}$ & Statistic & Date & $\begin{array}{c}\text { Time } \\
\text { (local) }\end{array}$ & $\begin{array}{c}\text { D0sat } \\
\text { (percent) }\end{array}$ & $\begin{array}{c}\text { DO } \\
\text { (mg/L) }\end{array}$ & $\begin{array}{c}\mathrm{DO}^{1} \\
\text { (ratio) }\end{array}$ & $\begin{array}{c}\mathrm{pH} \\
\text { (standard } \\
\text { units) }\end{array}$ & $\begin{array}{c}\text { SC } \\
(\mu \mathbf{S} / \mathbf{c m})\end{array}$ & $\begin{array}{c}\text { Water } \\
\text { temperature } \\
\text { ('C) }\end{array}$ & $\begin{array}{c}\text { Air } \\
\text { temperature } \\
\text { ('C) }\end{array}$ & $\begin{array}{c}\text { Barometric } \\
\text { pressure } \\
(\mathrm{mm} \mathrm{Hg})\end{array}$ \\
\hline \multirow{2}{*}{1} & AM & $07 / 27 / 1999$ & $04: 20$ & 62.8 & 5.1 & \multirow{2}{*}{1.9} & 7.7 & 185 & 25.84 & 22.0 & 730 \\
\hline & $\mathrm{PM}$ & 07/27/1999 & $14: 49$ & 126.9 & 9.9 & & 8.2 & 178 & 28.46 & 33.0 & - \\
\hline \multirow{2}{*}{2} & $\mathrm{AM}$ & 07/27/1999 & $04: 26$ & 71.9 & 6.0 & \multirow{2}{*}{1.6} & 7.7 & 278 & 24.14 & 22.0 & 730 \\
\hline & $\mathrm{PM}$ & 07/27/1999 & $14: 55$ & 129.5 & 9.9 & & 8.2 & 307 & 29.13 & - & 730 \\
\hline \multirow{2}{*}{3} & $\mathrm{AM}$ & 07/27/1999 & 04:59 & 54.1 & 4.4 & \multirow{2}{*}{1.1} & 7.6 & 301 & 25.77 & 22.0 & 730 \\
\hline & $\mathrm{PM}$ & 07/27/1999 & $15: 08$ & 61.7 & 5.0 & & 7.8 & 255 & 26.54 & 33.0 & 730 \\
\hline \multirow{2}{*}{4} & $\mathrm{AM}$ & 07/27/1999 & $05: 15$ & 70.5 & 5.7 & \multirow{2}{*}{1.6} & 7.9 & 274 & 25.24 & 22.0 & 730 \\
\hline & $\mathrm{PM}$ & 07/27/1999 & $15: 17$ & 122.0 & 9.3 & & 8.8 & 244 & 29.42 & 33.0 & 730 \\
\hline \multirow{2}{*}{5} & $\mathrm{AM}$ & 07/27/1999 & $05: 25$ & 44.3 & 3.6 & \multirow{2}{*}{2.4} & 7.8 & 284 & 25.01 & 21.0 & 731 \\
\hline & $\mathrm{PM}$ & 07/27/1999 & $15: 23$ & 111.0 & 8.7 & & 8.3 & 281 & 28.06 & 33.0 & 730 \\
\hline \multirow{2}{*}{7} & $\mathrm{AM}$ & 07/27/1999 & $05: 46$ & 41.8 & 3.5 & \multirow{2}{*}{3.4} & 7.8 & 365 & 24.62 & 21.5 & 730 \\
\hline & PM & 07/27/1999 & $15: 28$ & 149.6 & 11.8 & & 8.4 & 360 & 27.79 & 33.0 & 730 \\
\hline \multirow{2}{*}{8} & $\mathrm{AM}$ & 07/27/1999 & 06:07 & 82.0 & 6.7 & \multirow{2}{*}{1.8} & 7.9 & 308 & 25.66 & 22.0 & - \\
\hline & $\mathrm{PM}$ & 07/27/1999 & $15: 52$ & 150.7 & 11.8 & & 8.5 & 343 & 27.78 & 33.0 & 730 \\
\hline \multirow{2}{*}{9} & $\mathrm{AM}$ & 07/27/1999 & $06: 15$ & 72.3 & 5.9 & \multirow{2}{*}{ - } & 7.9 & 426 & 25.74 & 22.0 & - \\
\hline & $\mathrm{PM}$ & - & - & - & - & & - & - & - & - & - \\
\hline \multirow{2}{*}{10} & $\mathrm{AM}$ & 07/27/1999 & $06: 32$ & 43.0 & 3.6 & \multirow{2}{*}{3.9} & 7.8 & 778 & 25.21 & 21.0 & 732 \\
\hline & $\mathrm{PM}$ & 07/27/1999 & $16: 13$ & 184.5 & 14.0 & & 8.5 & 856 & 29.84 & 32.0 & 730 \\
\hline \multirow{2}{*}{11} & $\mathrm{AM}$ & 07/28/1999 & $04: 12$ & 97.1 & 7.8 & \multirow{2}{*}{1.3} & 8.2 & 663 & 26.49 & 23.0 & 732 \\
\hline & $\mathrm{PM}$ & 07/27/1999 & $16: 26$ & 132.7 & 10.3 & & 8.5 & 648 & 28.36 & 32.0 & - \\
\hline \multirow{2}{*}{12} & $\mathrm{AM}$ & 07/27/1999 & 07:05 & 30.9 & 2.6 & \multirow{2}{*}{3.8} & 7.8 & 780 & 24.11 & - & 732 \\
\hline & PM & 07/27/1999 & $16: 40$ & 126.8 & 9.8 & & 8.5 & 780 & 29.38 & - & 732 \\
\hline \multirow{2}{*}{14} & $\mathrm{AM}$ & 07/27/1999 & $07: 17$ & 25.1 & 2.1 & \multirow{2}{*}{3.0} & 7.7 & 756 & 23.78 & 22.0 & 732 \\
\hline & $\mathrm{PM}$ & 07/27/1999 & $16: 46$ & 80.8 & 6.3 & & 8.2 & 730 & 28.06 & 32.0 & 732 \\
\hline \multirow{2}{*}{15} & $\mathrm{AM}$ & 07/28/1999 & 04:21 & 49.2 & 4.3 & \multirow{2}{*}{2.9} & 7.8 & 544 & 22.67 & 23.0 & 732 \\
\hline & $\mathrm{PM}$ & 07/27/1999 & 17:04 & 150.8 & 12.3 & & 8.4 & 510 & 25.62 & 33.5 & 732 \\
\hline \multirow{2}{*}{16} & $\mathrm{AM}$ & 07/28/1999 & 04:30 & 47.7 & 4.0 & \multirow{2}{*}{2.8} & 7.7 & 462 & 24.27 & 33.0 & 732 \\
\hline & $\mathrm{PM}$ & 07/27/1999 & $17: 14$ & 139.0 & 11.0 & & 8.4 & 510 & 27.32 & 33.5 & 732 \\
\hline
\end{tabular}


Table 4. Continued.

\begin{tabular}{|c|c|c|c|c|c|c|c|c|c|c|c|}
\hline $\begin{array}{c}\text { Site } \\
\text { number } \\
\text { (Fig. 2) }\end{array}$ & Statistic & Date & $\begin{array}{c}\text { Time } \\
\text { (local) }\end{array}$ & $\begin{array}{c}\text { D0sat } \\
\text { (percent) }\end{array}$ & $\begin{array}{c}\text { DO } \\
\text { (mg/L) }\end{array}$ & $\begin{array}{c}\mathrm{DO}^{1} \\
\text { (ratio) }\end{array}$ & $\begin{array}{c}\text { pH } \\
\text { (standard } \\
\text { units) }\end{array}$ & $\begin{array}{c}\text { SC } \\
(\mu \mathbf{S} / \mathbf{c m})\end{array}$ & $\begin{array}{c}\text { Water } \\
\text { temperature } \\
\text { ('C) }\end{array}$ & $\begin{array}{c}\text { Air } \\
\text { temperature } \\
\left.\text { ( }{ }^{\circ} \mathrm{C}\right)\end{array}$ & $\begin{array}{c}\text { Barometric } \\
\text { pressure } \\
\text { (mm Hg) }\end{array}$ \\
\hline \multirow{2}{*}{17} & AM & $07 / 28 / 1999$ & $04: 40$ & 65.0 & 5.3 & \multirow{2}{*}{2.0} & 7.9 & 514 & 25.47 & 23.0 & 732 \\
\hline & $\mathrm{PM}$ & 07/27/1999 & $17: 32$ & 137.6 & 10.8 & & 8.4 & 516 & 27.93 & 33.0 & 732 \\
\hline \multirow{2}{*}{18} & $\mathrm{AM}$ & 07/28/1999 & $04: 50$ & 47.1 & 3.9 & \multirow{2}{*}{2.5} & 7.8 & 482 & 25.58 & 23.0 & 732 \\
\hline & $\mathrm{PM}$ & 07/29/1999 & $14: 30$ & 120.8 & 9.8 & & 8.3 & 496 & 24.31 & 31.5 & 730 \\
\hline \multirow{2}{*}{21} & $\mathrm{AM}$ & 07/28/1999 & 05:08 & 33.3 & 2.7 & \multirow{2}{*}{3.3} & 7.7 & 458 & 25.41 & 23.0 & 732 \\
\hline & $\mathrm{PM}$ & 07/29/1999 & $14: 42$ & 112.1 & 9.0 & & 8.3 & 432 & 26.62 & 31.5 & 730 \\
\hline \multirow{2}{*}{24} & $\mathrm{AM}$ & $07 / 28 / 1999$ & $05: 28$ & 41.0 & 3.4 & \multirow{2}{*}{3.5} & 7.8 & 447 & 24.56 & 22.0 & 736 \\
\hline & $\mathrm{PM}$ & 07/29/1999 & $14: 55$ & 150.9 & 12.1 & & 8.4 & 430 & 26.39 & 32.5 & 730 \\
\hline \multirow{2}{*}{25} & $\mathrm{AM}$ & 07/28/1999 & $05: 46$ & 27.7 & 2.3 & \multirow{2}{*}{3.4} & 7.8 & 444 & 25.30 & 22.0 & 236 \\
\hline & $\mathrm{PM}$ & 07/29/1999 & $15: 10$ & 98.3 & 7.9 & & 8.3 & 422 & 26.82 & 32.5 & 730 \\
\hline \multirow{2}{*}{26} & $\mathrm{AM}$ & $07 / 28 / 1999$ & 05:50 & 39.7 & 3.8 & \multirow{2}{*}{3.5} & 7.7 & 464 & 17.00 & 22.0 & 736 \\
\hline & $\mathrm{PM}$ & 07/29/1999 & $15: 26$ & 149.9 & 13.3 & & 8.3 & 232 & 21.13 & 32.5 & 732 \\
\hline \multirow{2}{*}{31} & $\mathrm{AM}$ & 07/28/1999 & 06:18 & 64.4 & 5.5 & \multirow{2}{*}{2.2} & 7.8 & 456 & 22.87 & 22.0 & 739 \\
\hline & $\mathrm{PM}$ & 07/29/1999 & $15: 38$ & 152.0 & 12.1 & & 8.6 & 448 & 27.02 & 32.5 & 732 \\
\hline \multirow{2}{*}{33} & $\mathrm{AM}$ & $07 / 28 / 1999$ & $06: 29$ & 45.5 & 3.7 & \multirow{2}{*}{3.3} & 8.1 & 452 & 25.38 & 22.0 & 740 \\
\hline & $\mathrm{PM}$ & 07/29/1999 & $16: 02$ & 154.9 & 12.3 & & 8.6 & 440 & 27.06 & 33.0 & 733 \\
\hline \multirow{2}{*}{35} & $\mathrm{AM}$ & 07/28/1999 & 05:50 & 51.4 & 4.2 & \multirow{2}{*}{3.0} & 7.9 & 427 & 25.83 & 26.0 & 736 \\
\hline & PM & 07/29/1999 & $16: 20$ & 166.4 & 12.6 & & 8.9 & 413 & 29.79 & 33.5 & 732 \\
\hline \multirow{2}{*}{36} & $\mathrm{AM}$ & 07/28/1999 & $05: 40$ & 73.6 & 5.9 & \multirow{2}{*}{2.0} & 8.5 & 419 & 26.88 & 25.5 & - \\
\hline & $\mathrm{PM}$ & 07/29/1999 & $16: 35$ & 151.6 & 11.9 & & 8.8 & 409 & 28.04 & 33.0 & 733 \\
\hline \multirow{2}{*}{39} & $\mathrm{AM}$ & 07/28/1999 & 05:10 & 95.2 & 7.5 & \multirow{2}{*}{1.3} & 8.9 & 437 & 27.59 & 25.0 & 737 \\
\hline & $\mathrm{PM}$ & 07/29/1999 & $16: 00$ & 124.1 & 9.7 & & 8.8 & 434 & 27.85 & 34.0 & 733 \\
\hline \multirow{2}{*}{40} & $\mathrm{AM}$ & 07/28/1999 & $04: 40$ & 60.5 & 4.9 & \multirow{2}{*}{3.0} & 8.9 & 440 & 26.35 & 25.5 & 738 \\
\hline & $\mathrm{PM}$ & 07/29/1999 & $15: 45$ & 189.1 & 14.5 & & 9.1 & 426 & 29.19 & 32.5 & 734 \\
\hline \multirow{2}{*}{41} & $\mathrm{AM}$ & 07/28/1999 & 04:15 & 58.0 & 4.7 & \multirow{2}{*}{3.3} & 8.6 & 455 & 26.23 & 24.5 & 738 \\
\hline & $\mathrm{PM}$ & 07/29/1999 & $15: 35$ & 202.2 & 15.3 & & 9.3 & 416 & 29.74 & 33.0 & 734 \\
\hline \multirow{2}{*}{42} & $\mathrm{AM}$ & $07 / 27 / 1999$ & $06: 40$ & 76.0 & 6.1 & \multirow{2}{*}{1.7} & 9.3 & 412 & 26.92 & 24.5 & 738 \\
\hline & PM & 07/27/1999 & $17: 40$ & 132.4 & 10.3 & & 9.0 & 445 & 28.21 & 35.0 & 735 \\
\hline 13 & $\mathrm{AM}$ & 07/27/1999 & 05:50 & 68.8 & 5.6 & 20 & 8.9 & 425 & 25.95 & 23.5 & 740 \\
\hline 43 & $\mathrm{PM}$ & 07/27/1999 & $17: 10$ & 149.5 & 11.4 & 2.0 & 9.6 & 390 & 29.52 & 35.0 & 736 \\
\hline
\end{tabular}


Table 4. Continued.

\begin{tabular}{|c|c|c|c|c|c|c|c|c|c|c|c|}
\hline $\begin{array}{c}\text { Site } \\
\text { number } \\
\text { (Fig. 2) }\end{array}$ & Statistic & Date & $\begin{array}{c}\text { Time } \\
\text { (local) }\end{array}$ & $\begin{array}{c}\text { DOsat } \\
\text { (percent) }\end{array}$ & $\begin{array}{c}\text { DO } \\
\text { (mg/L) }\end{array}$ & $\begin{array}{c}\mathrm{DO}^{1} \\
\text { (ratio) }\end{array}$ & $\begin{array}{c}\mathrm{pH} \\
\text { (standard } \\
\text { units) }\end{array}$ & $\begin{array}{c}\text { SC } \\
(\mu \mathrm{S} / \mathrm{cm})\end{array}$ & $\begin{array}{c}\text { Water } \\
\text { temperature } \\
\text { ('C) }\end{array}$ & $\begin{array}{c}\text { Air } \\
\text { temperature } \\
\text { (ㄷ) }\end{array}$ & $\begin{array}{c}\text { Barometric } \\
\text { pressure } \\
(\mathrm{mm} \mathrm{Hg})\end{array}$ \\
\hline \multirow{2}{*}{44} & AM & $07 / 27 / 1999$ & $05: 58$ & 56.4 & 4.5 & \multirow{2}{*}{2.8} & 9.0 & 417 & 26.47 & 23.5 & - \\
\hline & PM & 07/27/1999 & $17: 15$ & 163.2 & 12.5 & & 9.5 & 392 & 29.48 & 34.5 & - \\
\hline \multirow{2}{*}{45} & AM & 07/27/1999 & 05:30 & 55.1 & 4.4 & \multirow{2}{*}{3.3} & 9.0 & 415 & 26.78 & 23.0 & - \\
\hline & PM & 07/27/1999 & 17:00 & 193.2 & 14.6 & & 9.5 & 392 & 30.14 & 37.0 & 736 \\
\hline \multirow{2}{*}{46} & AM & 07/27/1999 & 05:05 & 87.9 & 7.0 & \multirow{2}{*}{1.5} & 9.3 & 397 & 27.41 & 22.5 & 738 \\
\hline & PM & 07/27/1999 & $16: 40$ & 132.2 & 10.3 & & 9.3 & 400 & 28.45 & 33.0 & 737 \\
\hline \multirow{2}{*}{47} & AM & 07/27/1999 & 04:40 & 58.7 & 4.7 & \multirow{2}{*}{3.0} & 9.1 & 413 & 26.39 & 22.0 & - \\
\hline & PM & 07/27/1999 & $16: 21$ & 184.2 & 14.0 & & 9.5 & 405 & 29.58 & 34.5 & 737 \\
\hline \multirow{2}{*}{52} & $\mathrm{AM}$ & 07/27/1999 & 04:20 & 73.6 & 5.8 & \multirow{2}{*}{1.5} & 8.9 & 403 & 27.15 & 21.5 & 739 \\
\hline & PM & 07/27/1999 & 16:05 & 111.2 & 8.6 & & 9.0 & 414 & 28.82 & 33.5 & 738 \\
\hline
\end{tabular}

${ }^{1}$ Calculated by dividing the late afternoon dissolved-oxygen concentration by the early morning dissolved-oxygen concentration 


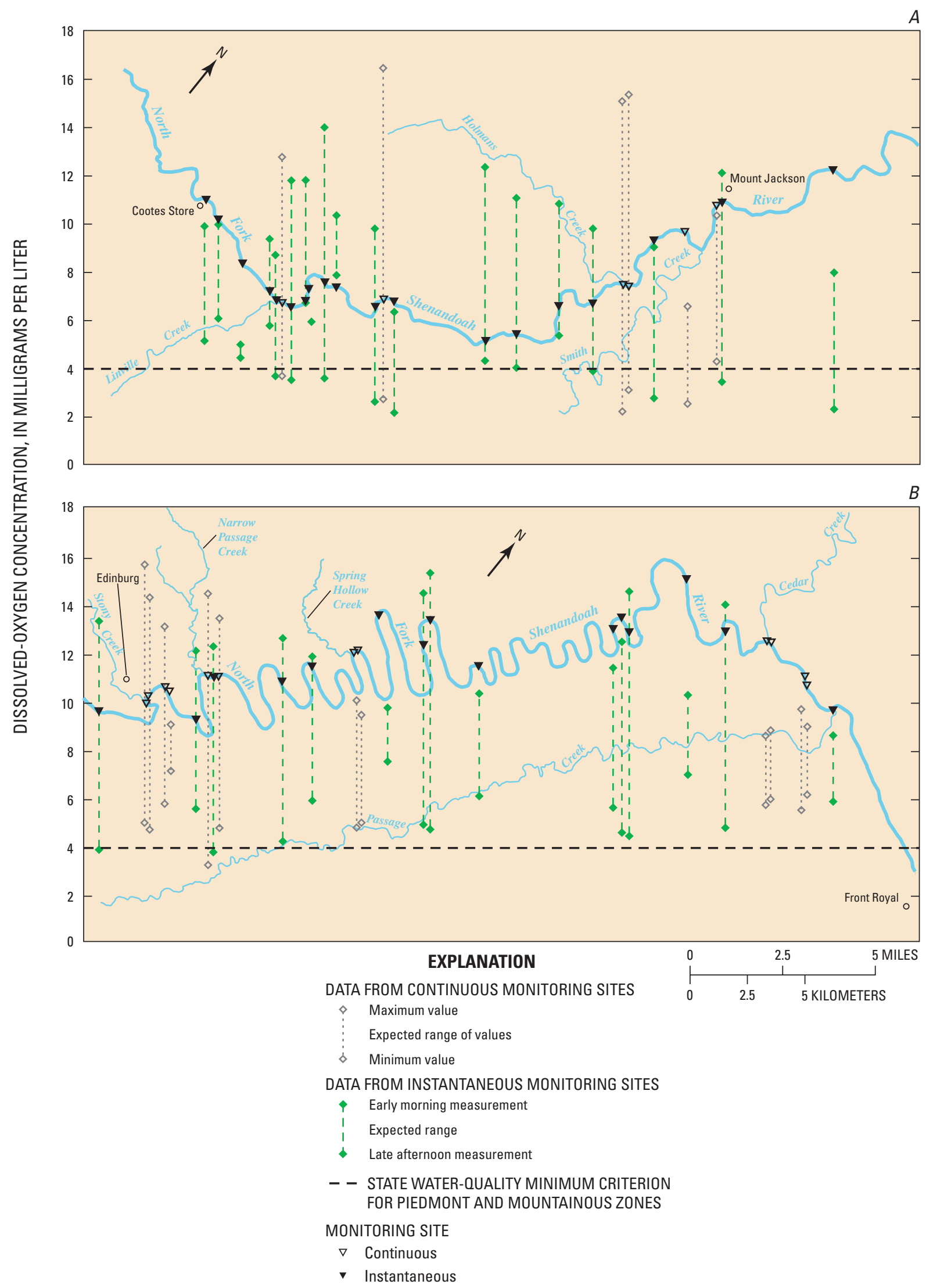

Figure 4. Dissolved-oxygen concentrations at water-quality monitoring sites in sections $A$ and $B$ of the North Fork Shenandoah River, Virginia. 


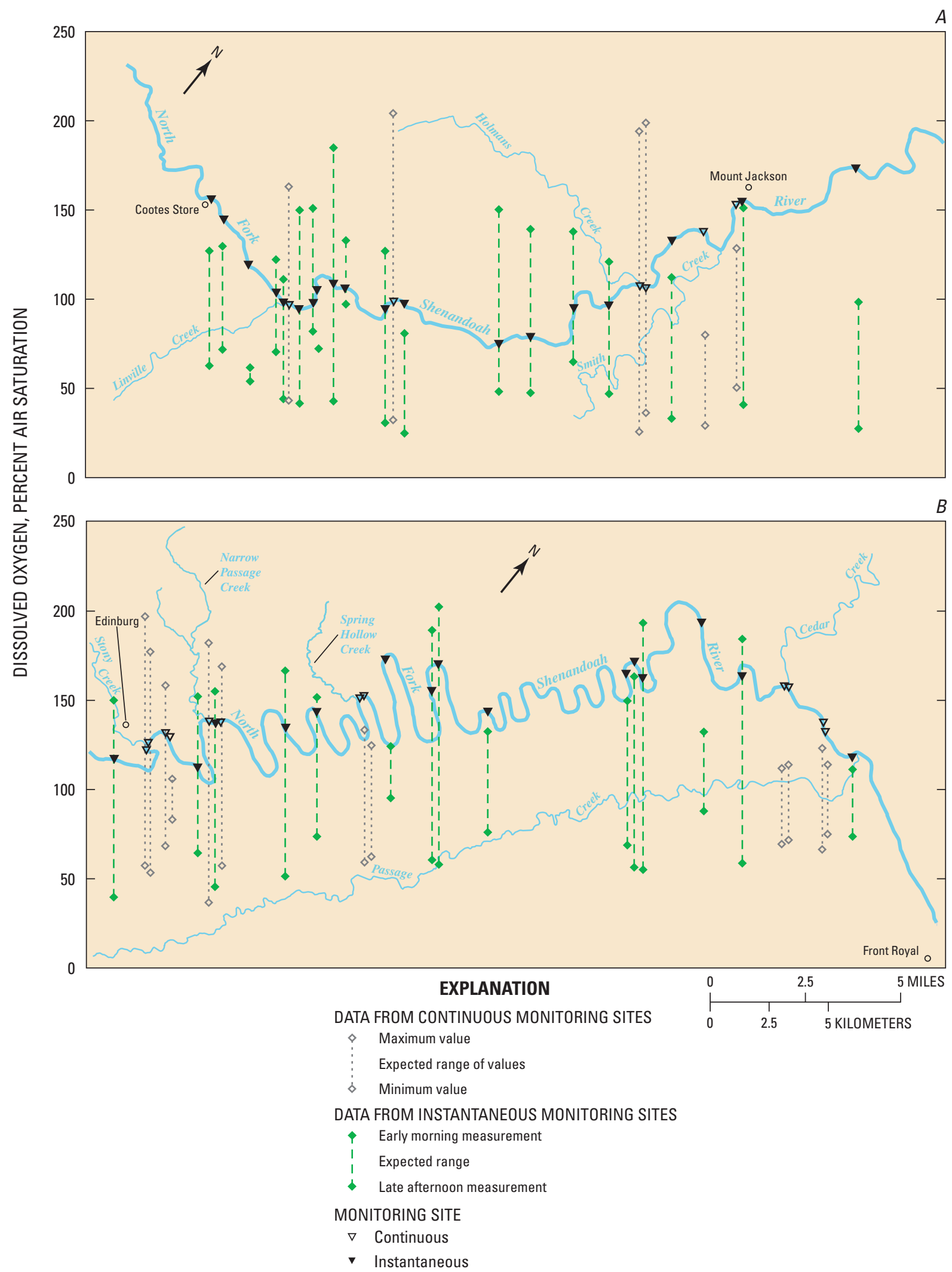

Figure 5. Dissolved-oxygen as percent air saturation at water-quality monitoring sites in sections $A$ and $B$ of the North Fork Shenandoah River, Virginia. 
Although there were no significant differences between datasets, the range of values recorded during the continuous sampling (2.2-16.4 $\mathrm{mg} / \mathrm{L})$ was greater than the range of values from the instantaneous sampling $(2.1-15.3 \mathrm{mg} / \mathrm{L})$. The dissolved-oxygen ratios (fig. 6) represent the maximum dissolved-oxygen concentration divided by the minimum dissolved-oxygen concentration at each monitoring site. The larger the ratio, the greater the difference between the maximum and minimum dissolved-oxygen concentrations. Ratios can be used to compare daily fluctuations of dissolved-oxygen concentrations.

At continuous water-quality monitoring sites, dissolved-oxygen concentrations fluctuated as much as $10 \mathrm{mg} / \mathrm{L}$ per day, and typically fluctuated at least $5 \mathrm{mg} / \mathrm{L}$ per day during the study period. The daily fluctuations of dissolved-oxygen concentrations for selected pairs of continuous water-quality monitoring sites (sites 19 and 20, 32 and 34, and 37 and 38) are illustrated (fig. 7 and app.). At instantaneous water-quality monitoring sites, the mean daily fluctuation of dissolved-oxygen concentrations was approximately $6 \mathrm{mg} / \mathrm{L}$. Daily fluctuations of dissolved-oxygen concentrations have been recently documented on the Colorado River below Glen Canyon Dam, Ariz. (Flynn and others, 2001), on the White River near Sumner, Wash. (Ebbert, 2003), and on six rivers within south-central Texas (Ging and Otero, 2003). The previously mentioned studies report daily fluctuations in the range of $5 \mathrm{mg} / \mathrm{L}$. Photosynthesis and respiration of the benthic organisms affect the daily fluctuation of dissolved-oxygen concentration and $\mathrm{pH}$ in rivers (Hem, 1989; Marzolf and others, 1999; Flynn and others, 2001). Summer low flows and high water temperatures on the North Fork Shenandoah River may have contributed to excessive algal growth, which may affect the wide range of daily fluctuation of dissolved-oxygen concentrations.

\section{pH}

At all sites, $\mathrm{pH}$ was slightly alkaline, with values ranging from 7.6 to 9.6 (fig. 8, tables 3 and 4). The greatest $\mathrm{pH}$ values were found between sites 40 and 52 in the Seven Bends area of the river (fig. 2). The State numerical criteria for the minimum and maximum values of $\mathrm{pH}$ in Piedmont and Mountainous zones are 6.0 and 9.0, respectively (State Water Control Board, 2002). Upstream of site 40, at Rittenour Ridge (fig. 2), all measured $\mathrm{pH}$ values were less than 9.0, but from Rittenour Ridge downstream to site 52, $\mathrm{pH}$ values greater than 9.0 were measured at all sites (fig. 8). Most semi-hourly values from continuous water-quality monitoring sites were less than 9.0, but at sites 48, 49, 50, and 51, pH values greater than 9.0 were measured on multiple days (app.). River water generally has a $\mathrm{pH}$ in the range of 6.5-8.5 (Hem, 1989). When photosynthesis by aquatic organisms takes up dissolved carbon dioxide during the day, and the organisms release carbon dioxide at night through respiration, $\mathrm{pH}$ fluctuation may occur, and the maximum $\mathrm{pH}$ value can reach as high as 9.0 (Stumm and Morgan, 1981; Hem, 1989). On the White River near Sumner, Wash., the minimum and the maximum $\mathrm{pH}$ values ranged from approximately 7.5 to 8.5 daily in September and October 2002 (Ebbert, 2003). As turbidity increased in the White River, the daily fluctuation of $\mathrm{pH}$ decreased, demonstrating the effect photosynthesis has on daily fluctuation of $\mathrm{pH}$ in streams (Ebbert, 2003). On the North Fork Shenandoah River, the karst topography over the limestone and dolomite rocks may contribute to the greater $\mathrm{pH}$ of river water in this area, but the maximum $\mathrm{pH}$ values measured were likely affected by photosynthesis and respiration as well.

\section{Specific Conductance}

Specific-conductance values from both continuous and instantaneous water-quality monitoring sites ranged from 178 to $856 \mu \mathrm{S} / \mathrm{cm}$ (fig. 9). No State numerical criteria have been established for specific-conductance values. Most specific-conductance values were less than $550 \mu \mathrm{S} / \mathrm{cm}$. From sites 10 to 14 , specific-conductance values were greater than $600 \mu \mathrm{S} / \mathrm{cm}$, and ranged from 648 to $856 \mu \mathrm{S} / \mathrm{cm}$. Daily fluctuations of specific conductance of 10 to $30 \mu \mathrm{S} / \mathrm{cm}$ were typical at continuous and instantaneous water-quality monitoring sites.

\section{Temperature}

Air and water temperature serve as a reference to climactic conditions during the study period. Air temperature data were collected only from the 34 instantaneous water-quality monitoring sites. Air temperatures ranged from 21.0 to $37.0^{\circ} \mathrm{C}$ (table 4 ). These air temperatures are typical for summer months in this region. Water temperatures from both continuous and instantaneous water-quality monitoring sites ranged from 17.00 to $30.14^{\circ} \mathrm{C}$ (fig. 10 , tables 3 and 4 ). As would be expected during a summer low-flow period, water temperature was above $25^{\circ} \mathrm{C}$ at least once at 50 sites. The State numerical criterion for the maximum water temperature in Piedmont and Mountainous zones is $31.0^{\circ} \mathrm{C}$ (State Water Control Board, 2002). All measured water temperatures were below $31.0^{\circ} \mathrm{C}$ (fig. 10), except for some of the semi-hourly values collected at continuous water-quality monitoring site 37 , where a maximum water temperature of $31.10^{\circ} \mathrm{C}$ was measured on July 23,1999 (app.). The complete range of values from the semi-hourly temperature dataset is reported in the appendix. A water temperature of $17.00^{\circ} \mathrm{C}$ was measured at site 26 , an instantaneous monitoring site (section B, fig. 10). This value is less than all other measured water temperatures; however, during field data collection it was noted that a spring discharged to the river at this site. Because ground-water temperatures are typically colder than surface-water temperatures in the summer, it is likely that the water temperature measured at site 26 was affected by groundwater discharge from the spring. 


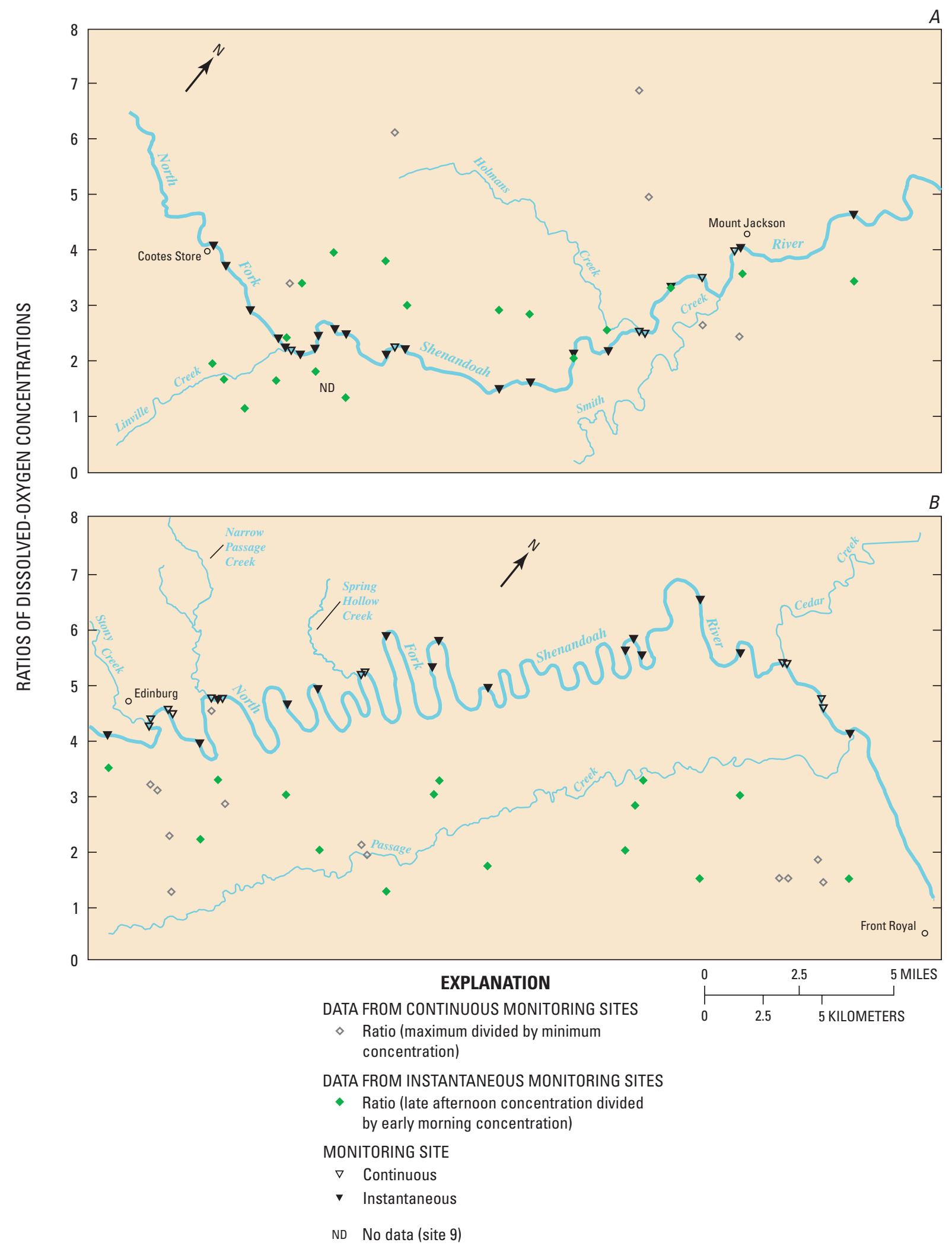

Figure 6. Ratios of dissolved-oxygen concentrations at water-quality monitoring sites in sections $A$ and $B$ of the North Fork Shenandoah River, Virginia. 


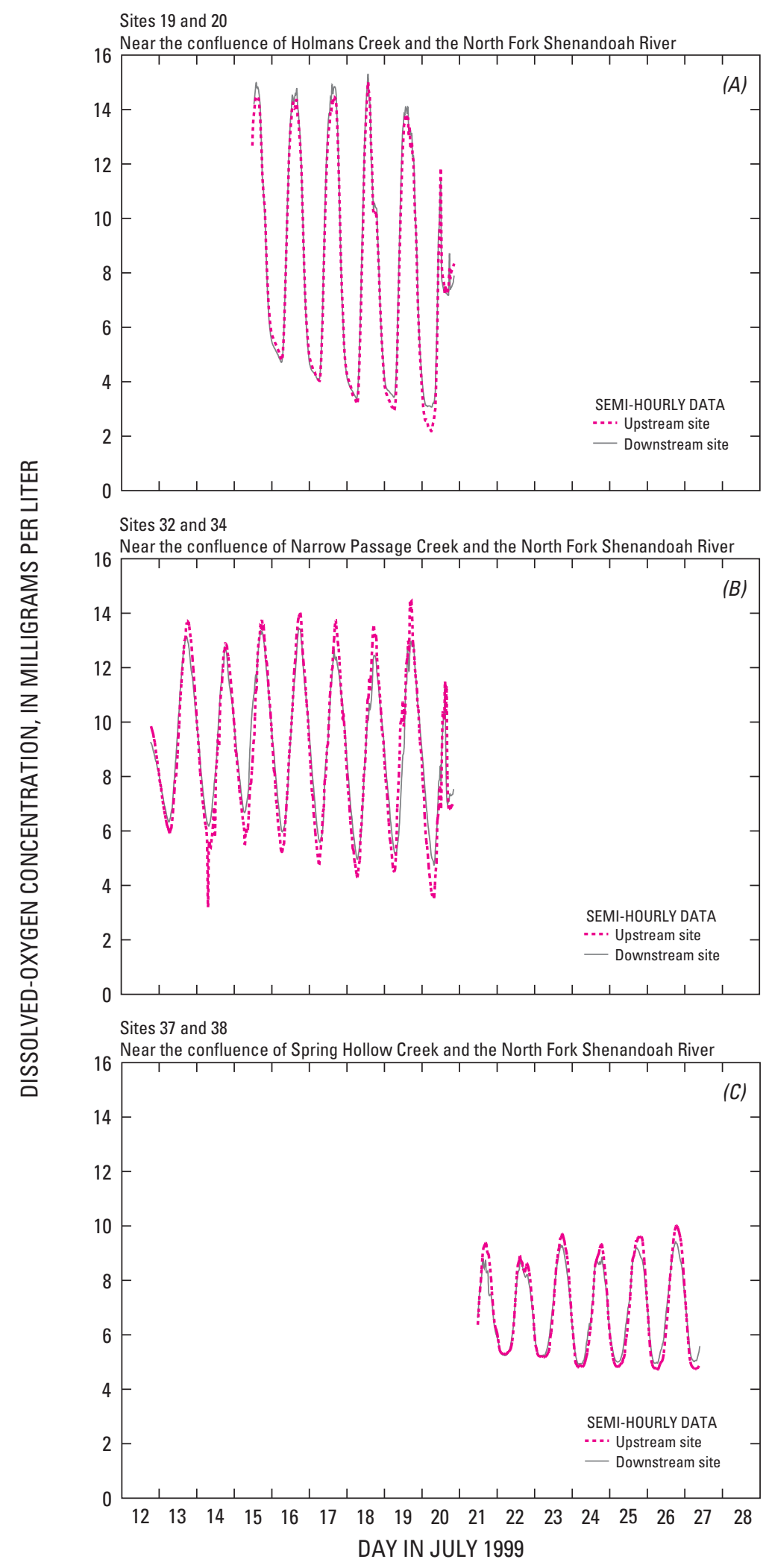

Figure 7. Dissolved-oxygen concentrations at three pairs of upstream and downstream continuous water-quality monitoring sites along the North Fork Shenandoah River, Virginia, during July 15-20 (A), July 12-20 (B), and July 21-27 (C), 1999. 


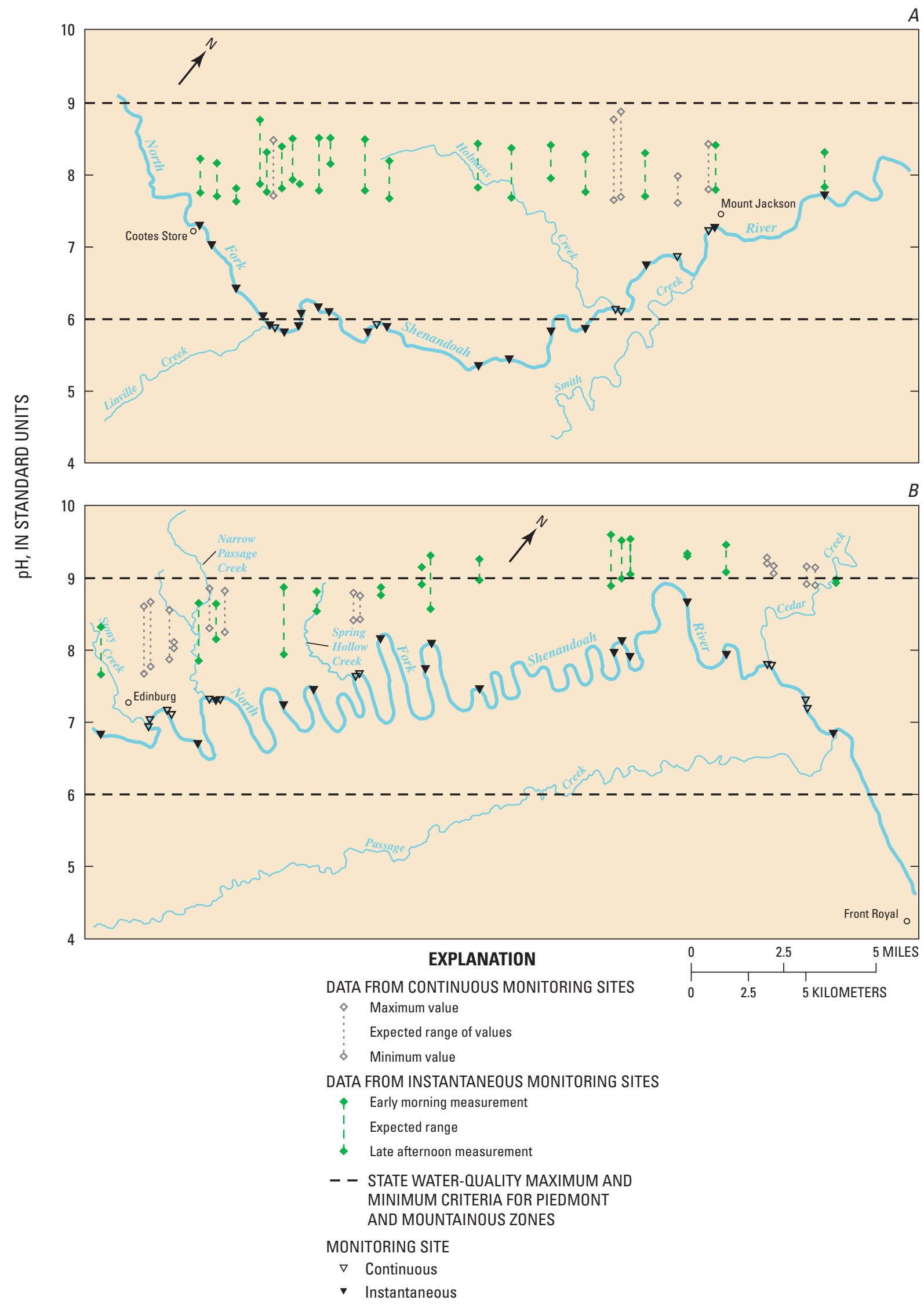

Figure 8. $\mathrm{pH}$ at water-quality monitoring sites in sections $A$ and $B$ of the North Fork Shenandoah River, Virginia. 


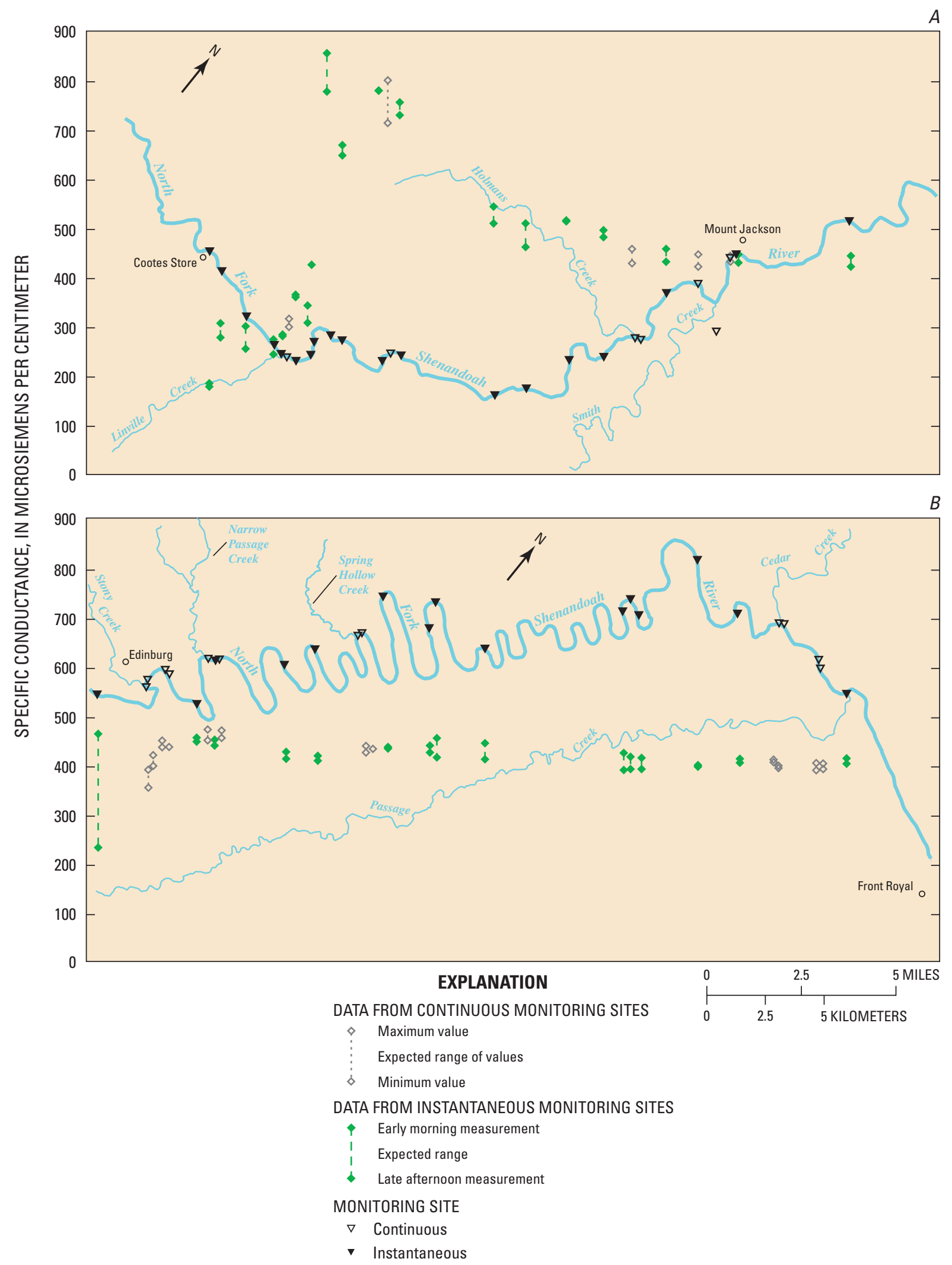

Figure 9. Specific conductance at water-quality monitoring sites in sections A and B of the North Fork Shenandoah River, Virginia. 


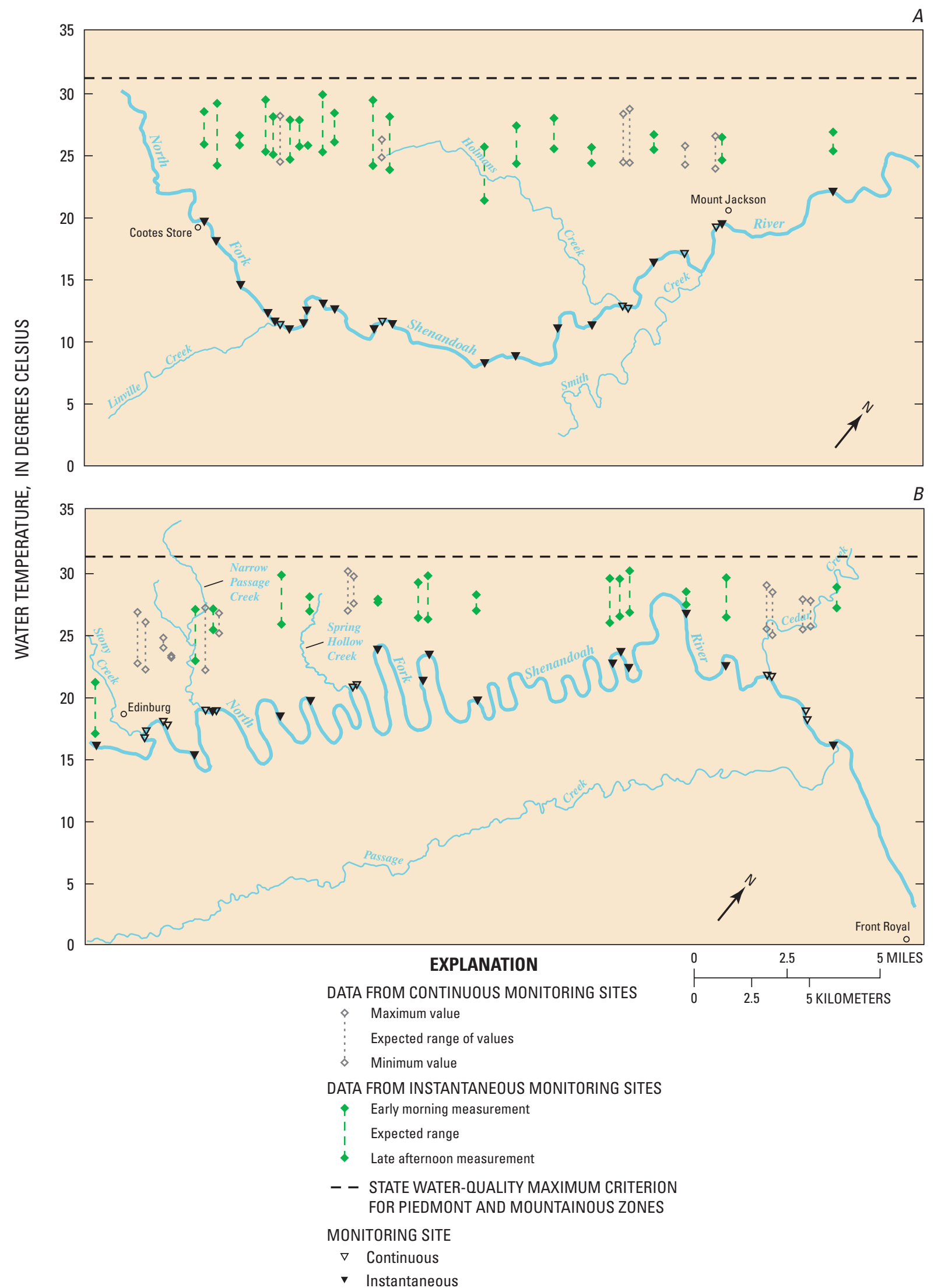

Figure 10. Water temperature at water-quality monitoring sites in sections $A$ and $B$ of the North Fork Shenandoah River, Virginia. 


\section{Summary and Conclusions}

Increased demand for water resources, in conjunction with population growth, has brought about widespread concern about water availability within the North Fork Shenandoah River Basin, Virginia. In a rural basin with the potential for urban and industrial growth, the availability of a clean, abundant water supply is a primary concern. There is also concern for maintaining streamflows that are adequate to support the aquatic community, to support recreation, and to maintain aesthetic characteristics of the river. Mean monthly flows in July 1999 recorded at three streamflow-gaging stations on the river were the lowest recorded in the past 55- to 74-year periods of record. Recent droughts have increased public awareness of the importance of a sustainable water supply and have increased demand for water-availability and water-quality information to assist with water-resources management in the basin.

In 1998, an Instream Flow Incremental Methodology (IFIM) study was initiated by the USGS, in cooperation with the Northern Shenandoah Valley Regional Commission and Virginia Polytechnic Institute and State University, to incorporate hydrologic information, habitat requirements of fish, and wateruse information into a comprehensive assessment of the water resources of the North Fork Shenandoah River Basin. As a part of the IFIM study, water-quality data were collected in July of 1999 to provide information about water-quality conditions to which aquatic life are exposed during summer low-flow periods (defined as flows less than the 90-percent exceedance flow). The study focused on dissolved-oxygen concentrations because the variability of dissolved-oxygen concentrations can affect fish health.

Dissolved-oxygen concentration, $\mathrm{pH}$, specific conductance, and water temperature were measured at 52 sites along 80 mi of the North Fork Shenandoah River from Cootes Store, Va., to its confluence with Passage Creek, near Strasburg, Va. Maximum and minimum dissolved-oxygen concentrations were determined for 18 sites with continuous water-quality monitors. These data represent the greatest (MAX) and least (MIN) semihourly concentrations of dissolved oxygen recorded during the study period and are the upper and lower limits of the range of values measured. Dissolved-oxygen concentrations also were measured at 34 sites in the early morning (AM) and late afternoon (PM) with hand-held water-quality monitors to best approximate the daily minimum and daily maximum dissolvedoxygen concentrations, respectively.

Dissolved-oxygen concentrations ranged from 2.1 to $16.4 \mathrm{mg} / \mathrm{L}$. Dissolved oxygen as percent air saturation ranged from 25 to 204 percent. Daily fluctuations of dissolved-oxygen concentrations were at least $5 \mathrm{mg} / \mathrm{L}$ at each site. Dissolved-oxygen concentrations were equal to or less than the State waterquality minimum of $4.0 \mathrm{mg} / \mathrm{L}$ at 18 of 52 monitoring sites; all 18 sites were in the upper and middle portions of the river, where more than half of the first 34 sites had minimum dissolved-oxygen concentrations equal to or less than $4.0 \mathrm{mg} / \mathrm{L}$.
The $\mathrm{pH}$ was slightly alkaline at all sites, and ranged from 7.6 to 9.6 , with $\mathrm{pH}$ values greater than 9.0 measured at the 13 sites farthest downstream. The State numerical criteria for the minimum and maximum values of $\mathrm{pH}$ in Piedmont and Mountainous zones are 6.0 and 9.0, respectively. One-hundred percent of $\mathrm{pH}$ values measured were greater than the State minimum, and 75 percent of $\mathrm{pH}$ values measured were less than the State maximum during the study period. More information is needed about the combined effects of geology, ground-water discharge to the river, primary production, and human impacts to the river, to determine the cause of the maximum $\mathrm{pH}$ values on the North Fork Shenandoah River. Specific-conductance values ranged from 178 to $856 \mu \mathrm{S} / \mathrm{cm}$, with values greater than $600 \mu \mathrm{S} / \mathrm{cm}$ measured at a group of 5 sites in the upper portion of the river. Air temperatures ranged from 21.0 to $37.0^{\circ} \mathrm{C}$, and water temperatures ranged from 17.00 to $30.14^{\circ} \mathrm{C}$. The State numerical criterion for the maximum water temperature in Piedmont and Mountainous zones is $31.0^{\circ} \mathrm{C}$. At 51 sites, measured water temperatures were below $31.0^{\circ} \mathrm{C}$.

Water-quality data in this report serve as baseline information on summer, extreme low-flow conditions on the North Fork Shenandoah River. The flows observed are not within the normal range of flows for summer months, and are in part the result of reduced precipitation over the previous 12 months. However, water-quality conditions vary seasonally and annually, depending on the hydrologic condition, and periodically, extremely low-flow conditions result. The dissolved-oxygen concentrations measured during this study showed great daily fluctuations, and were less than the State minimum criteria at 18 sites. $\mathrm{pH}$ values exceeded State numerical criteria for maximum value of $\mathrm{pH}$ at 13 sites. To understand the degree to which the water-quality conditions observed in this study are representative, and to understand how the water-quality conditions recorded in this study can affect the aquatic community, it would be best to have a comparable dataset from a summer period within a normal range of flows. Further study would be required to assess the duration of conditions similar to those reported in this study, and any changes in water-quality conditions that result from increased water withdrawals from the North Fork Shenandoah River. 


\section{References Cited}

Davis, J.C., 1975, Minimal dissolved oxygen requirements of aquatic life with special emphasis on Canadian species-a review: Journal of Fisheries Research Board of Canada, v. 32, p. 2295-2332.

Ebbert, J.C., 2003, Water temperature, specific conductance, $\mathrm{pH}$, and dissolved-oxygen concentrations in the Lower White River and the Puyallup River Estuary, Washington, AugustOctober 2002: U.S. Geological Survey Water-Resources Investigations Report 03-4177, 21 p.

Flynn, M.E., Hart, R.J., Marzolf, G.R., and Bowser, C.J., 2001, Daily and seasonal variability of $\mathrm{pH}$, dissolved oxygen, temperature, and specific conductance in the Colorado River between the forebay of Glen Canyon Dam and Lees Ferry, northeastern Arizona, 1989-99: U.S. Geological Survey Water-Resources Investigations Report 01-4240, 13 p.

Fry, F.E.J., 1971, The effect of environmental factors on physiology, in Hoar, W.S., and Randall, D.J., eds., Fish physiology, v. VI: New York, Academic Press, Inc., p. 1-87.

Ging, P.B., and Otero, C.L., 2003, Comparison of temperature, specific conductance, $\mathrm{pH}$, and dissolved oxygen at selected basic fixed sites in south-central Texas, 1996-98: U.S. Geological Survey Open-File Report 03-087, 18 p.

Hack, J.T., 1965, Geomorphology of the Shenandoah Valley, Virginia and West Virginia, and origin of the residual ore deposits: U.S. Geological Survey Professional Paper 484, $84 \mathrm{p}$.

Hayden, B.P., and Michaels, P.J., Virginia State Climatology Office, 2003, Virginia's climate: Virginia State Climatology Office, accessed September 2003 at URL http://climate.virginia.edu/description.htm

Hayes, D.C., 1991, Low-flow characteristics of streams in Virginia: U.S. Geological Survey Water-Supply Paper 2374, $69 \mathrm{p}$.

Holeton G.F., and Randall, D.J., 1967, The effect of hypoxia upon the partial pressure of gases in the blood and water afferent and efferent to the gills of rainbow trout: Journal of Experimental Biology, v. 46, p. 317-327.

Hutson, S.S., Barber, N.L., Kenny, J.F., Linsey, K.S., Lumia, D.S., and Maupin, M.A., 2004, Estimated use of water in the United States in 2000: U.S. Geological Survey Circular 1268, $46 \mathrm{p}$.

Leonard, P.M., Orth, D.J., and Goudreau, C.J., 1986, Development of a method for recommending instream flows for fishes in the upper James River, Virginia: Blacksburg, Va., Virginia Water Resources Research Center, Virginia Polytechnic Institute and State University Bulletin 152, 122 p.

Orth, D.J., and Leonard, P.M., 1990, Comparison of discharge methods and habitat optimization for recommending instream flows to protect fish habitat: Regulated Rivers: Research and Management, v. 5, p. 129-138.

Ott, R.L., and Longnecker, Michael, 2001, An introduction to statistical methods and data analysis ( $5^{\text {th }}$ ed.): Pacific Grove, Calif., Duxbury, 1152 p.
Persinger, J.W., 2003, Developing habitat suitability criteria for individual species and habitat guilds in the Shenandoah River Basin: Blacksburg, Va., Virginia Polytechnic Institute and State University, Master's Thesis, 207 p.

Roble, S.M., 2003, Natural heritage resources of Virginia-rare animal species, Natural Heritage Technical Report 03-04: Richmond, Va., Virginia Department of Conservation and Recreation, Division of Natural Heritage, 39 p. [Searchable on-line database accessed September 1, 2004, available at URL http://192.206.31.52/cfprog/dnh/naturalheritage/ select watershed.cfm]

Searcy, J.K., 1969, Flow-duration curves, manual of hydrology: Part 2. Low-flow techniques: U.S. Geological Survey WaterSupply Paper 1542-A, 33 p.

Solley, W.B., Merk, C.F., and Pierce, R.R., 1988, Estimated use of water in the United States in 1985: U.S. Geological Survey Circular 1004, 82 p.

Solley, W.B., Pierce, R.R., and Perlman, H.A., 1993, Estimated use of water in the United States in 1990: U.S. Geological Survey Circular 1081, 76 p.

Solley, W.B., Pierce, R.R., and Perlman, H.A., 1998, Estimated use of water in the United States in 1995: U.S. Geological Survey Circular 1200, $71 \mathrm{p}$.

Southeast Regional Climate Center, 2003, Climate interactive rapid retrieval users system: precipitation and temperature data accessed September 2003 at URL http:// www.dnr.state.sc.us/pls/cirrus/cirrus.home [Data available by subscription only as of March 2004, available at URL http://www.dnr.state.sc.us/climate/sercc/index.html, or available through the U.S. Geological Survey, Virginia District office]

Stalnaker, C.B., 1981, Low flow as a limiting factor in warmwater streams: American Fisheries Society, Warmwater Streams Symposium, p. 192-199.

State Water Control Board, 2002, Part 1, Surface water standards with general, statewide application, 9 VAC 25-260-50 Numerical criteria for dissolved oxygen, $\mathrm{pH}$, and maximum temperature, in 9 VAC 25-260 Virginia water quality standards: Virginia Department of Environmental Quality, accessed August 27, 2002, at URL http:// www.deq.state.va.us/wqs/homepage.html

Stewart, N.E., Shumway, D.L., and Doudoroff, Peter, 1967, Influence of oxygen concentration on the growth of juvenile largemouth bass: Journal of Fisheries Research Board of Canada, v. 24, p. 475-494.

Stumm, Werner, and Morgan, J.J., 1981, Aquatic chemistryintroduction emphasizing chemical equilibria in natural waters, $2^{\text {nd }}$ ed.: New York, John Wiley and Sons, 780 p.

Vadas, R.L., Jr., and Weigmann, D.L., 1993, The concept of instream flow and its relevance to drought management in the James River basin: Blacksburg, Va., Virginia Water Resources Research Center, Virginia Polytechnic Institute and State University Bulletin 182, $78 \mathrm{p}$.

U.S. Census Bureau, 2002, Table 1 of Ranking tables for counties-population in 2000 and population change from 1990 to 2000 (PHC-T-4): U.S. Census Bureau data available on the 
Web, accessed November 2003 at URL http://www.census.gov/population/www/cen2000/phc-t4.html

U.S. Environmental Protection Agency, 1996, EPA Region III land cover dataset: version 2, GIS grid available on the Web, accessed January 6, 2004, at URL http://landcover.usgs.gov/ nlcd/show data.asp?code $=$ VA\&state $=$ Virginia

U.S. Geological Survey, 1997 to present, National field manual for the collection of water-quality data: U.S. Geological Survey Techniques of Water-Resources Investigations, book 9, chaps. A1-A9, 2 v., variously paged. [Also available online at URL http://pubs.water.usgs.gov/twri9A. Chapters originally were published from 1997 to 1999; updates and revisions are ongoing and are summarized at URL http:// water.usgs.gov/owq/FieldManual/mastererrata.html]

Virginia Department of Conservation and Economic Development, 1968, Potomac-Shenandoah River Basin, Comprehensive water resources plan: Division of Water Resources, Planning Bulletin 207, v. I, 157 p.

White, R.K., Hayes, D.C., Eckenwiler, M.R., and Herman, P.E., 2000, Water resources data, Virginia, water year 1999: U.S. Geological Survey Water-Data Report VA-99-1, 456 p.

Whitworth, W.R., 1968, Effects of diurnal fluctuations of dissolved oxygen on the growth of brook trout: Journal of Fisheries Research Board of Canada, v. 25, p. 579-584.

Wilding, J.L., 1939, The oxygen threshold for three species of fish: Ecology, v. 20, no. 2, p. 253-263.

YSI Incorporated, 1998, Environmental monitoring systems operations manual: 6-series, Revision A, Yellow Springs, Ohio, $255 \mathrm{p}$.

Zappia, Humbert, and Hayes, D.C., 1998, A demonstration of the instream flow incremental methodology, Shenandoah River, Virginia: U.S. Geological Survey Water-Resources Investigations Report 98-4157, 24 p. 

Appendix

$\longrightarrow$ 
Appendix Continuous water-quality monitoring data for the North Fork Shenandoah River, Virginia

[See table 1 for site names and descriptions; see figure 2 for site locations; ${ }^{\circ} \mathrm{C}$, degrees Celsius; SC, specific conductance; $\mu \mathrm{S} / \mathrm{cm}$, microsiemens per centimeter; DOsat, dissolved oxygen as percent air saturation; DO, dissolved oxygen; mg/L, milligrams per liter; -, no data]

\begin{tabular}{|c|c|c|c|c|c|c|}
\hline Date & Time & $\begin{array}{c}\text { Water } \\
\text { temperature } \\
\text { ('C) }\end{array}$ & $\begin{array}{c}\text { SC } \\
(\mu \mathbf{S} / \mathbf{c m})\end{array}$ & $\begin{array}{c}\text { DOsat } \\
\text { (percent) }\end{array}$ & $\begin{array}{c}\text { DO } \\
\text { (mg/L) }\end{array}$ & $\begin{array}{c}\mathrm{pH} \\
\text { (standard } \\
\text { units) }\end{array}$ \\
\hline \multicolumn{7}{|c|}{ Site 6} \\
\hline 7/29/1999 & $14: 30: 40$ & 26.77 & 302 & 101.7 & 8.1 & 8.0 \\
\hline 7/29/1999 & $15: 00: 40$ & 26.93 & 302 & 100.6 & 8.0 & 8.0 \\
\hline $7 / 29 / 1999$ & $15: 30: 40$ & 27.43 & 300 & 109.2 & 8.6 & 8.1 \\
\hline $7 / 29 / 1999$ & $16: 00: 40$ & 27.41 & 301 & 115.6 & 9.1 & 8.2 \\
\hline $7 / 29 / 1999$ & $16: 30: 40$ & 27.59 & 302 & 130.4 & 10.3 & 8.2 \\
\hline $7 / 29 / 1999$ & $17: 00: 40$ & 27.72 & 304 & 132.4 & 10.4 & 8.3 \\
\hline $7 / 29 / 1999$ & $17: 30: 40$ & 28.06 & 309 & 149.4 & 11.7 & 8.4 \\
\hline $7 / 29 / 1999$ & 18:00:40 & 28.12 & 316 & 162.6 & $12.7^{\mathrm{a}}$ & 8.5 \\
\hline $7 / 29 / 1999$ & $18: 30: 40$ & 28.41 & 316 & 158.1 & 12.3 & 8.5 \\
\hline $7 / 29 / 1999$ & 19:00:40 & 28.35 & 315 & 159.1 & 12.4 & 8.5 \\
\hline $7 / 29 / 1999$ & $19: 30: 40$ & 28.41 & 310 & 161.0 & 12.5 & 8.6 \\
\hline $7 / 29 / 1999$ & 20:00:40 & 28.74 & 300 & 149.5 & 11.5 & 8.6 \\
\hline $7 / 29 / 1999$ & $20: 30: 40$ & 29.29 & 269 & 113.5 & 8.7 & 8.6 \\
\hline $7 / 29 / 1999$ & 21:00:40 & 29.3 & 267 & 102.1 & 7.8 & 8.4 \\
\hline $7 / 29 / 1999$ & $21: 30: 40$ & 29.16 & 265 & 93.7 & 7.2 & 8.3 \\
\hline $7 / 29 / 1999$ & $22: 00: 40$ & 28.92 & 264 & 85.2 & 6.6 & 8.3 \\
\hline $7 / 29 / 1999$ & $22: 30: 40$ & 28.62 & 265 & 81.9 & 6.3 & 8.3 \\
\hline $7 / 29 / 1999$ & $23: 00: 40$ & 28.28 & 266 & 76.4 & 6.0 & 8.2 \\
\hline 7/29/1999 & $23: 30: 40$ & 27.91 & 268 & 70.4 & 5.5 & 8.1 \\
\hline $7 / 30 / 1999$ & 0:00:40 & 27.55 & 271 & 65.3 & 5.2 & 8.0 \\
\hline $7 / 30 / 1999$ & 0:30:40 & 27.19 & 273 & 61.2 & 4.9 & 7.9 \\
\hline $7 / 30 / 1999$ & 1:00:40 & 26.85 & 276 & 57.5 & 4.6 & 7.9 \\
\hline $7 / 30 / 1999$ & $1: 30: 40$ & 26.54 & 279 & 54.4 & 4.4 & 7.8 \\
\hline $7 / 30 / 1999$ & 2:00:40 & 26.26 & 281 & 52.0 & 4.2 & 7.8 \\
\hline 7/30/1999 & $2: 30: 40$ & 26 & 283 & 49.8 & 4.0 & 7.8 \\
\hline $7 / 30 / 1999$ & 3:00:40 & 25.77 & 285 & 48.3 & 3.9 & 7.8 \\
\hline $7 / 30 / 1999$ & $3: 30: 40$ & 25.55 & 286 & 47.4 & 3.9 & 7.8 \\
\hline $7 / 30 / 1999$ & 4:00:40 & 25.34 & 287 & 47.0 & 3.9 & 7.7 \\
\hline $7 / 30 / 1999$ & 4:30:40 & 25.15 & 289 & 46.8 & 3.9 & 7.7 \\
\hline $7 / 30 / 1999$ & 5:00:40 & 24.97 & 290 & 46.7 & 3.9 & 7.7 \\
\hline $7 / 30 / 1999$ & $5: 30: 40$ & 24.82 & 291 & 46.3 & 3.8 & 7.7 \\
\hline $7 / 30 / 1999$ & 6:00:40 & 24.69 & 292 & 45.4 & 3.8 & 7.7 \\
\hline $7 / 30 / 1999$ & $6: 30: 40$ & 24.58 & 293 & 44.5 & 3.7 & 7.7 \\
\hline $7 / 30 / 1999$ & 7:00:40 & 24.48 & 294 & 44.0 & 3.7 & 7.7 \\
\hline 7/30/1999 & 7:30:40 & 24.4 & 295 & 43.7 & $3.7^{\mathbf{b}}$ & 7.7 \\
\hline $7 / 30 / 1999$ & 8:00:40 & 24.38 & 296 & 44.9 & 3.8 & 7.7 \\
\hline $7 / 30 / 1999$ & $8: 30: 40$ & 24.42 & 296 & 48.1 & 4.0 & 7.7 \\
\hline 7/30/1999 & 9:00:40 & 24.41 & 297 & 50.9 & 4.3 & 7.7 \\
\hline $7 / 30 / 1999$ & 9:30:40 & 24.42 & 297 & 53.3 & 4.5 & 7.8 \\
\hline 7/30/1999 & 10:00:40 & 24.71 & 297 & 60.7 & 5.0 & 7.8 \\
\hline \multicolumn{7}{|c|}{ Site 13} \\
\hline $7 / 29 / 1999$ & $12: 00: 40$ & 24.11 & 761 & 136.3 & 11.4 & - \\
\hline $7 / 29 / 1999$ & $12: 30: 40$ & 24.54 & 758 & 157.2 & 13.1 & - \\
\hline
\end{tabular}


Appendix Continued

\begin{tabular}{|c|c|c|c|c|c|c|}
\hline Date & Time & $\begin{array}{c}\text { Water } \\
\text { temperature } \\
\left.\text { ( }{ }^{\circ} \mathrm{C}\right)\end{array}$ & $\begin{array}{c}\text { SC } \\
(\mu \mathbf{S} / \mathbf{c m})\end{array}$ & $\begin{array}{c}\text { DOsat } \\
\text { (percent) }\end{array}$ & $\begin{array}{c}\text { DO } \\
\text { (mg/L) }\end{array}$ & $\begin{array}{c}\text { pH } \\
\text { (standard } \\
\text { units) }\end{array}$ \\
\hline $7 / 29 / 1999$ & $13: 00: 40$ & 24.76 & 750 & 167.7 & 13.9 & - \\
\hline $7 / 29 / 1999$ & $13: 30: 40$ & 25.1 & 743 & 181.0 & 14.9 & - \\
\hline 7/29/1999 & 14:00:40 & 25.45 & 730 & 197.9 & 16.2 & - \\
\hline 7/29/1999 & $14: 30: 40$ & 26.21 & 713 & 203.5 & $16.4^{\mathrm{a}}$ & - \\
\hline $7 / 29 / 1999$ & $15: 00: 40$ & 27.26 & 703 & 205.0 & 16.2 & - \\
\hline $7 / 29 / 1999$ & $15: 30: 40$ & 28.41 & 727 & 194.2 & 15.1 & - \\
\hline $7 / 29 / 1999$ & $16: 00: 40$ & 28.68 & 729 & 188.1 & 14.5 & - \\
\hline $7 / 29 / 1999$ & $16: 30: 40$ & 28.61 & 729 & 182.6 & 14.1 & - \\
\hline $7 / 29 / 1999$ & $17: 00: 40$ & 28.6 & 726 & 181.5 & 14.0 & - \\
\hline $7 / 29 / 1999$ & $17: 30: 40$ & 28.6 & 726 & 177.6 & 13.7 & - \\
\hline $7 / 29 / 1999$ & 18:00:40 & 28.52 & 725 & 171.8 & 13.3 & - \\
\hline $7 / 29 / 1999$ & $18: 30: 40$ & 28.41 & 726 & 163.9 & 12.7 & - \\
\hline $7 / 29 / 1999$ & 19:00:40 & 27.97 & 733 & 150.3 & 11.8 & - \\
\hline $7 / 29 / 1999$ & $19: 30: 40$ & 27.71 & 739 & 136.7 & 10.7 & - \\
\hline $7 / 29 / 1999$ & $20: 00: 40$ & 27.52 & 746 & 121.1 & 9.5 & - \\
\hline $7 / 29 / 1999$ & $20: 30: 40$ & 27.38 & 752 & 104.6 & 8.3 & - \\
\hline $7 / 29 / 1999$ & 21:00:40 & 27.26 & 760 & 88.6 & 7.0 & - \\
\hline $7 / 29 / 1999$ & $21: 30: 40$ & 27.01 & 765 & 76.7 & 6.1 & - \\
\hline $7 / 29 / 1999$ & $22: 00: 40$ & 26.72 & 772 & 68.3 & 5.5 & - \\
\hline $7 / 29 / 1999$ & $22: 30: 40$ & 26.46 & 776 & 61.4 & 4.9 & - \\
\hline $7 / 29 / 1999$ & $23: 00: 40$ & 26.27 & 782 & 55.5 & 4.5 & - \\
\hline $7 / 29 / 1999$ & $23: 30: 40$ & 26.07 & 787 & 50.2 & 4.1 & - \\
\hline $7 / 30 / 1999$ & 0:00:40 & 25.82 & 791 & 46.2 & 3.8 & - \\
\hline $7 / 30 / 1999$ & $0: 30: 40$ & 25.61 & 793 & 43.7 & 3.6 & - \\
\hline 7/30/1999 & 1:00:40 & 25.59 & 798 & 40.5 & 3.3 & - \\
\hline 7/30/1999 & $1: 30: 40$ & 25.43 & 802 & 38.2 & 3.1 & - \\
\hline 7/30/1999 & 2:00:40 & 25.2 & 801 & 35.9 & 3.0 & - \\
\hline 7/30/1999 & $2: 30: 40$ & 24.98 & 800 & 33.9 & 2.8 & - \\
\hline 7/30/1999 & 3:00:40 & 24.75 & 800 & 32.9 & $2.7^{\mathbf{b}}$ & - \\
\hline $7 / 30 / 1999$ & $3: 30: 40$ & 24.55 & 799 & 34.9 & 2.9 & - \\
\hline 7/30/1999 & 4:00:40 & 24.35 & 797 & 34.7 & 2.9 & - \\
\hline 7/30/1999 & 4:30:40 & 24.19 & 795 & 35.3 & 3.0 & - \\
\hline 7/30/1999 & 5:00:40 & 24.06 & 794 & 35.2 & 3.0 & - \\
\hline 7/30/1999 & $5: 30: 40$ & 23.95 & 792 & 35.2 & 3.0 & - \\
\hline 7/30/1999 & 6:00:40 & 23.81 & 791 & 37.7 & 3.2 & - \\
\hline 7/30/1999 & $6: 30: 40$ & 23.73 & 790 & 37.6 & 3.2 & - \\
\hline 7/30/1999 & 7:00:40 & 23.69 & 788 & 40.4 & 3.4 & - \\
\hline 7/30/1999 & 7:30:40 & 23.64 & 787 & 44.4 & 3.8 & - \\
\hline 7/30/1999 & 8:00:40 & 23.63 & 786 & 48.4 & 4.1 & - \\
\hline 7/30/1999 & $8: 30: 40$ & 23.67 & 784 & 57.2 & 4.8 & - \\
\hline 7/30/1999 & 9:00:40 & 23.75 & 782 & 62.3 & 5.3 & - \\
\hline 7/30/1999 & $9: 30: 40$ & 23.79 & 782 & 63.6 & 5.4 & - \\
\hline \multicolumn{7}{|c|}{ Site 19} \\
\hline $7 / 15 / 1999$ & 11:30:00 & 23.92 & 477 & 150.4 & 12.7 & 8.5 \\
\hline $7 / 15 / 1999$ & 12:00:40 & 24.37 & 460 & 161.9 & 13.5 & 8.6 \\
\hline $7 / 15 / 1999$ & $12: 30: 40$ & 24.81 & 457 & 167.3 & 13.9 & 8.6 \\
\hline $7 / 15 / 1999$ & $13: 00: 40$ & 25.24 & 453 & 172.0 & 14.1 & 8.7 \\
\hline $7 / 15 / 1999$ & $13: 30: 40$ & 25.57 & 450 & 175.8 & 14.4 & 8.7 \\
\hline $7 / 15 / 1999$ & $14: 00: 40$ & 25.86 & 447 & 178.2 & 14.5 & 8.7 \\
\hline
\end{tabular}


Water-Quality Synoptic Sampling, July 1999: North Fork Shenandoah River, Virginia

Appendix Continued

\begin{tabular}{|c|c|c|c|c|c|c|}
\hline Date & Time & $\begin{array}{c}\text { Water } \\
\text { temperature } \\
\text { ('C) }\end{array}$ & $\begin{array}{c}\text { SC } \\
(\mu \mathbf{S} / \mathbf{c m})\end{array}$ & $\begin{array}{c}\text { DOsat } \\
\text { (percent) }\end{array}$ & $\begin{array}{c}\mathrm{DO} \\
(\mathrm{mg} / \mathrm{L})\end{array}$ & $\begin{array}{c}\text { pH } \\
\text { (standard } \\
\text { units) }\end{array}$ \\
\hline $7 / 15 / 1999$ & $14: 30: 40$ & 26.04 & 444 & 178.5 & 14.5 & 8.7 \\
\hline 7/15/1999 & $15: 00: 40$ & 26.22 & 441 & 178.3 & 14.4 & 8.7 \\
\hline 7/15/1999 & $15: 30: 40$ & 26.31 & 439 & 178.6 & 14.4 & 8.7 \\
\hline $7 / 15 / 1999$ & $16: 00: 40$ & 26.27 & 437 & 175.1 & 14.1 & 8.7 \\
\hline $7 / 15 / 1999$ & $16: 30: 40$ & 26.19 & 436 & 169.7 & 13.7 & 8.7 \\
\hline 7/15/1999 & $17: 00: 40$ & 25.96 & 437 & 159.9 & 13.0 & 8.6 \\
\hline 7/15/1999 & $17: 30: 40$ & 25.78 & 437 & 153.4 & 12.5 & 8.6 \\
\hline $7 / 15 / 1999$ & 18:00:40 & 25.51 & 440 & 141.7 & 11.6 & 8.6 \\
\hline $7 / 15 / 1999$ & $18: 30: 40$ & 25.36 & 442 & 134.6 & 11.0 & 8.5 \\
\hline $7 / 15 / 1999$ & 19:00:40 & 25.24 & 444 & 130.1 & 10.7 & 8.5 \\
\hline $7 / 15 / 1999$ & $19: 30: 40$ & 25.04 & 446 & 125.8 & 10.4 & 8.5 \\
\hline $7 / 15 / 1999$ & 20:00:40 & 24.75 & 449 & 115.0 & 9.5 & 8.4 \\
\hline 7/15/1999 & $20: 30: 40$ & 24.45 & 453 & 103.2 & 8.6 & 8.3 \\
\hline $7 / 15 / 1999$ & 21:00:40 & 24.22 & 457 & 93.8 & 7.9 & 8.2 \\
\hline $7 / 15 / 1999$ & $21: 30: 40$ & 24.04 & 460 & 86.3 & 7.3 & 8.1 \\
\hline $7 / 15 / 1999$ & $22: 00: 40$ & 23.91 & 462 & 80.6 & 6.8 & 8.1 \\
\hline $7 / 15 / 1999$ & $22: 30: 40$ & 23.82 & 465 & 75.9 & 6.4 & 8.0 \\
\hline $7 / 15 / 1999$ & $23: 00: 40$ & 23.75 & 466 & 72.2 & 6.1 & 8.0 \\
\hline $7 / 15 / 1999$ & $23: 30: 40$ & 23.68 & 467 & 69.7 & 5.9 & 8.0 \\
\hline 7/16/1999 & 0:00:40 & 23.63 & 468 & 68.1 & 5.8 & 8.0 \\
\hline $7 / 16 / 1999$ & 0:30:40 & 23.58 & 468 & 66.7 & 5.7 & 7.9 \\
\hline $7 / 16 / 1999$ & 1:00:40 & 23.53 & 469 & 65.7 & 5.6 & 7.9 \\
\hline $7 / 16 / 1999$ & $1: 30: 40$ & 23.48 & 469 & 64.9 & 5.5 & 7.9 \\
\hline 7/16/1999 & 2:00:40 & 23.44 & 470 & 63.9 & 5.4 & 7.9 \\
\hline $7 / 16 / 1999$ & $2: 30: 40$ & 23.41 & 470 & 63.5 & 5.4 & 7.9 \\
\hline $7 / 16 / 1999$ & 3:00:40 & 23.36 & 471 & 62.4 & 5.3 & 7.9 \\
\hline $7 / 16 / 1999$ & $3: 30: 40$ & 23.33 & 471 & 61.5 & 5.2 & 7.9 \\
\hline 7/16/1999 & 4:00:40 & 23.27 & 472 & 60.6 & 5.2 & 7.9 \\
\hline $7 / 16 / 1999$ & 4:30:40 & 23.24 & 472 & 59.8 & 5.1 & 7.9 \\
\hline $7 / 16 / 1999$ & 5:00:40 & 23.18 & 472 & 58.5 & 5.0 & 7.9 \\
\hline $7 / 16 / 1999$ & $5: 30: 40$ & 23.13 & 472 & 57.3 & 4.9 & 7.9 \\
\hline $7 / 16 / 1999$ & 6:00:40 & 23.08 & 473 & 56.1 & 4.8 & 7.9 \\
\hline $7 / 16 / 1999$ & $6: 30: 40$ & 23.03 & 473 & 56.0 & 4.8 & 7.9 \\
\hline $7 / 16 / 1999$ & 7:00:40 & 23.02 & 473 & 58.4 & 5.0 & 7.9 \\
\hline $7 / 16 / 1999$ & 7:30:40 & 23.06 & 473 & 64.6 & 5.5 & 7.9 \\
\hline $7 / 16 / 1999$ & 8:00:40 & 23.13 & 472 & 73.6 & 6.3 & 8.0 \\
\hline 7/16/1999 & $8: 30: 40$ & 23.28 & 471 & 84.8 & 7.2 & 8.1 \\
\hline $7 / 16 / 1999$ & 9:00:40 & 23.50 & 470 & 97.8 & 8.3 & 8.2 \\
\hline 7/16/1999 & $9: 30: 40$ & 23.77 & 467 & 111.1 & 9.4 & 8.3 \\
\hline $7 / 16 / 1999$ & $10: 00: 40$ & 24.08 & 465 & 123.7 & 10.4 & 8.3 \\
\hline $7 / 16 / 1999$ & $10: 30: 40$ & 24.44 & 463 & 136.2 & 11.4 & 8.4 \\
\hline $7 / 16 / 1999$ & 11:00:40 & 24.84 & 460 & 147.4 & 12.2 & 8.5 \\
\hline $7 / 16 / 1999$ & 11:30:40 & 25.25 & 458 & 155.8 & 12.8 & 8.5 \\
\hline $7 / 16 / 1999$ & $12: 00: 40$ & 25.66 & 455 & 163.7 & 13.4 & 8.6 \\
\hline 7/16/1999 & $12: 30: 40$ & 26.00 & 454 & 168.6 & 13.7 & 8.6 \\
\hline $7 / 16 / 1999$ & $13: 00: 40$ & 26.47 & 451 & 175.5 & 14.1 & 8.7 \\
\hline $7 / 16 / 1999$ & $13: 30: 40$ & 26.71 & 450 & 178.0 & 14.2 & 8.7 \\
\hline $7 / 16 / 1999$ & $14: 00: 40$ & 26.82 & 449 & 176.3 & 14.1 & 8.7 \\
\hline $7 / 16 / 1999$ & $14: 30: 40$ & 27.04 & 448 & 176.6 & 14.0 & 8.7 \\
\hline $7 / 16 / 1999$ & $15: 00: 40$ & 27.21 & 447 & 179.2 & 14.2 & 8.7 \\
\hline 7/16/1999 & $15: 30: 40$ & 27.35 & 445 & 181.3 & 14.3 & 8.7 \\
\hline
\end{tabular}


Appendix Continued

\begin{tabular}{|c|c|c|c|c|c|c|}
\hline Date & Time & $\begin{array}{c}\text { Water } \\
\text { temperature } \\
\text { ('C) }\end{array}$ & $\begin{array}{c}\text { SC } \\
(\mu \mathbf{S} / \mathbf{c m})\end{array}$ & $\begin{array}{c}\text { DOsat } \\
\text { (percent) }\end{array}$ & $\begin{array}{c}\text { DO } \\
\text { (mg/L) }\end{array}$ & $\begin{array}{c}\text { pH } \\
\text { (standard } \\
\text { units) }\end{array}$ \\
\hline $7 / 16 / 1999$ & $16: 00: 40$ & 27.47 & 445 & 181.7 & 14.3 & 8.7 \\
\hline 7/16/1999 & $16: 30: 40$ & 27.32 & 445 & 173.2 & 13.7 & 8.7 \\
\hline 7/16/1999 & 17:00:40 & 27.34 & 445 & 172.1 & 13.6 & 8.7 \\
\hline 7/16/1999 & $17: 30: 40$ & 27.20 & 445 & 165.6 & 13.1 & 8.7 \\
\hline 7/16/1999 & 18:00:40 & 27.07 & 445 & 160.9 & 12.8 & 8.6 \\
\hline 7/16/1999 & $18: 30: 40$ & 26.87 & 447 & 153.3 & 12.2 & 8.6 \\
\hline 7/16/1999 & 19:00:40 & 26.69 & 449 & 144.3 & 11.6 & 8.6 \\
\hline $7 / 16 / 1999$ & $19: 30: 40$ & 26.44 & 451 & 132.9 & 10.7 & 8.5 \\
\hline 7/16/1999 & 20:00:40 & 26.14 & 455 & 119.9 & 9.7 & 8.4 \\
\hline 7/16/1999 & $20: 30: 40$ & 25.84 & 458 & 106.0 & 8.6 & 8.4 \\
\hline $7 / 16 / 1999$ & 21:00:40 & 25.58 & 461 & 93.9 & 7.7 & 8.3 \\
\hline $7 / 16 / 1999$ & $21: 30: 40$ & 25.37 & 464 & 84.2 & 6.9 & 8.2 \\
\hline 7/16/1999 & $22: 00: 40$ & 25.22 & 466 & 76.9 & 6.3 & 8.1 \\
\hline $7 / 16 / 1999$ & $22: 30: 40$ & 25.09 & 467 & 71.3 & 5.9 & 8.0 \\
\hline $7 / 16 / 1999$ & $23: 00: 40$ & 24.98 & 468 & 66.3 & 5.5 & 8.0 \\
\hline 7/16/1999 & $23: 30: 40$ & 24.87 & 468 & 62.5 & 5.2 & 7.9 \\
\hline $7 / 17 / 1999$ & 0:00:40 & 24.77 & 469 & 59.5 & 4.9 & 7.9 \\
\hline 7/17/1999 & 0:30:40 & 24.67 & 469 & 57.9 & 4.8 & 7.9 \\
\hline 7/17/1999 & 1:00:40 & 24.61 & 469 & 56.8 & 4.7 & 7.9 \\
\hline 7/17/1999 & $1: 30: 40$ & 24.53 & 469 & 55.7 & 4.6 & 7.9 \\
\hline 7/17/1999 & 2:00:40 & 24.46 & 469 & 54.2 & 4.5 & 7.9 \\
\hline $7 / 17 / 1999$ & $2: 30: 40$ & 24.39 & 468 & 53.0 & 4.4 & 7.8 \\
\hline 7/17/1999 & 3:00:40 & 24.32 & 468 & 52.6 & 4.4 & 7.8 \\
\hline 7/17/1999 & $3: 30: 40$ & 24.26 & 468 & 51.8 & 4.3 & 7.8 \\
\hline 7/17/1999 & 4:00:40 & 24.19 & 468 & 50.4 & 4.2 & 7.8 \\
\hline 7/17/1999 & 4:30:40 & 24.12 & 468 & 49.5 & 4.2 & 7.8 \\
\hline 7/17/1999 & 5:00:40 & 24.06 & 468 & 48.9 & 4.1 & 7.8 \\
\hline 7/17/1999 & $5: 30: 40$ & 23.98 & 467 & 48.5 & 4.1 & 7.8 \\
\hline $7 / 17 / 1999$ & $6: 00: 40$ & 23.93 & 467 & 48.2 & 4.1 & 7.8 \\
\hline 7/17/1999 & $6: 30: 40$ & 23.87 & 467 & 47.9 & 4.0 & 7.8 \\
\hline 7/17/1999 & 7:00:40 & 23.84 & 466 & 50.7 & 4.3 & 7.8 \\
\hline 7/17/1999 & $7: 30: 40$ & 23.88 & 465 & 57.4 & 4.8 & 7.9 \\
\hline 7/17/1999 & 8:00:40 & 23.97 & 464 & 67.2 & 5.7 & 7.9 \\
\hline 7/17/1999 & $8: 30: 40$ & 24.13 & 463 & 78.3 & 6.6 & 8.0 \\
\hline 7/17/1999 & 9:00:40 & 24.35 & 461 & 91.1 & 7.6 & 8.1 \\
\hline $7 / 17 / 1999$ & 9:30:40 & 24.65 & 459 & 106.0 & 8.8 & 8.2 \\
\hline 7/17/1999 & 10:00:40 & 25.00 & 456 & 120.4 & 9.9 & 8.3 \\
\hline 7/17/1999 & $10: 30: 40$ & 25.39 & 454 & 133.3 & 10.9 & 8.3 \\
\hline 7/17/1999 & 11:00:40 & 25.80 & 451 & 145.4 & 11.8 & 8.4 \\
\hline 7/17/1999 & 11:30:40 & 26.21 & 449 & 154.7 & 12.5 & 8.5 \\
\hline 7/17/1999 & 12:00:40 & 26.62 & 447 & 163.0 & 13.1 & 8.5 \\
\hline 7/17/1999 & $12: 30: 40$ & 26.93 & 445 & 167.3 & 13.3 & 8.6 \\
\hline $7 / 17 / 1999$ & $13: 00: 40$ & 27.20 & 443 & 169.8 & 13.5 & 8.6 \\
\hline 7/17/1999 & $13: 30: 40$ & 27.56 & 441 & 177.0 & 14.0 & 8.7 \\
\hline 7/17/1999 & 14:00:40 & 27.76 & 440 & 177.1 & 13.9 & 8.7 \\
\hline 7/17/1999 & $14: 30: 40$ & 28.16 & 438 & 184.0 & 14.4 & 8.7 \\
\hline 7/17/1999 & $15: 00: 40$ & 28.29 & 438 & 183.0 & 14.2 & 8.7 \\
\hline 7/17/1999 & $15: 30: 40$ & 28.57 & 436 & 186.7 & 14.5 & 8.8 \\
\hline 7/17/1999 & $16: 00: 40$ & 28.67 & 436 & 186.1 & 14.4 & 8.8 \\
\hline 7/17/1999 & $16: 30: 40$ & 28.83 & 435 & 187.1 & 14.4 & 8.8 \\
\hline
\end{tabular}


Water-Quality Synoptic Sampling, July 1999: North Fork Shenandoah River, Virginia

Appendix Continued

\begin{tabular}{|c|c|c|c|c|c|c|}
\hline Date & Time & $\begin{array}{c}\text { Water } \\
\text { temperature } \\
\text { ('C) }\end{array}$ & $\begin{array}{c}\text { SC } \\
(\mu \mathrm{S} / \mathrm{cm})\end{array}$ & $\begin{array}{c}\text { DOsat } \\
\text { (percent) }\end{array}$ & $\begin{array}{c}\text { DO } \\
\text { (mg/L) }\end{array}$ & $\begin{array}{c}\text { pH } \\
\text { (standard } \\
\text { units) }\end{array}$ \\
\hline $7 / 17 / 1999$ & $17: 00: 40$ & 28.94 & 434 & 185.7 & 14.3 & 8.8 \\
\hline 7/17/1999 & $17: 30: 40$ & 28.84 & 434 & 179.4 & 13.8 & 8.8 \\
\hline 7/17/1999 & 18:00:40 & 28.69 & 435 & 171.2 & 13.2 & 8.7 \\
\hline 7/17/1999 & $18: 30: 40$ & 28.36 & 437 & 158.5 & 12.3 & 8.7 \\
\hline 7/17/1999 & 19:00:40 & 28.04 & 440 & 143.7 & 11.2 & 8.6 \\
\hline 7/17/1999 & $19: 30: 40$ & 27.71 & 443 & 128.4 & 10.1 & 8.6 \\
\hline 7/17/1999 & 20:00:40 & 27.41 & 448 & 110.8 & 8.8 & 8.5 \\
\hline 7/17/1999 & $20: 30: 40$ & 27.10 & 451 & 97.6 & 7.8 & 8.4 \\
\hline 7/17/1999 & 21:00:40 & 26.82 & 454 & 87.6 & 7.0 & 8.3 \\
\hline 7/17/1999 & 21:30:40 & 26.55 & 456 & 78.4 & 6.3 & 8.2 \\
\hline 7/17/1999 & 22:00:40 & 26.30 & 457 & 70.4 & 5.7 & 8.1 \\
\hline 7/17/1999 & $22: 30: 40$ & 26.10 & 459 & 62.6 & 5.1 & 8.0 \\
\hline 7/17/1999 & 23:00:40 & 25.95 & 460 & 58.1 & 4.7 & 7.9 \\
\hline 7/17/1999 & $23: 30: 40$ & 25.82 & 460 & 55.1 & 4.5 & 7.9 \\
\hline $7 / 18 / 1999$ & 0:00:40 & 25.71 & 461 & 52.6 & 4.3 & 7.9 \\
\hline 7/18/1999 & $0: 30: 40$ & 25.62 & 461 & 50.7 & 4.1 & 7.8 \\
\hline 7/18/1999 & 1:00:40 & 25.54 & 462 & 49.8 & 4.1 & 7.8 \\
\hline $7 / 18 / 1999$ & $1: 30: 40$ & 25.46 & 461 & 48.4 & 4.0 & 7.8 \\
\hline $7 / 18 / 1999$ & 2:00:40 & 25.38 & 460 & 47.1 & 3.9 & 7.8 \\
\hline $7 / 18 / 1999$ & 2:30:40 & 25.29 & 460 & 46.7 & 3.8 & 7.8 \\
\hline 7/18/1999 & 3:00:40 & 25.19 & 460 & 45.1 & 3.7 & 7.8 \\
\hline 7/18/1999 & 3:30:40 & 25.10 & 460 & 43.1 & 3.6 & 7.8 \\
\hline 7/18/1999 & 4:00:40 & 25.03 & 460 & 42.2 & 3.5 & 7.8 \\
\hline 7/18/1999 & 4:30:40 & 24.96 & 460 & 41.8 & 3.5 & 7.8 \\
\hline $7 / 18 / 1999$ & 5:00:40 & 24.88 & 459 & 40.9 & 3.4 & 7.8 \\
\hline $7 / 18 / 1999$ & $5: 30: 40$ & 24.82 & 459 & 40.2 & 3.3 & 7.8 \\
\hline 7/18/1999 & 6:00:40 & 24.74 & 459 & 38.7 & 3.2 & 7.7 \\
\hline 7/18/1999 & 6:30:40 & 24.67 & 459 & 38.4 & 3.2 & 7.7 \\
\hline 7/18/1999 & 7:00:40 & 24.64 & 458 & 40.0 & 3.3 & 7.7 \\
\hline 7/18/1999 & 7:30:40 & 24.64 & 457 & 46.9 & 3.9 & 7.8 \\
\hline 7/18/1999 & 8:00:40 & 24.71 & 457 & 57.4 & 4.8 & 7.8 \\
\hline 7/18/1999 & 8:30:40 & 24.86 & 456 & 70.2 & 5.8 & 7.9 \\
\hline 7/18/1999 & 9:00:40 & 25.05 & 454 & 83.0 & 6.9 & 8.0 \\
\hline 7/18/1999 & $9: 30: 40$ & 25.26 & 452 & 98.7 & 8.1 & 8.1 \\
\hline 7/18/1999 & 10:00:40 & 25.53 & 450 & 112.8 & 9.2 & 8.2 \\
\hline 7/18/1999 & $10: 30: 40$ & 25.84 & 447 & 129.8 & 10.6 & 8.3 \\
\hline 7/18/1999 & 11:00:40 & 26.20 & 444 & 145.0 & 11.7 & 8.4 \\
\hline 7/18/1999 & $11: 30: 40$ & 26.60 & 441 & 158.1 & 12.7 & 8.5 \\
\hline 7/18/1999 & $12: 00: 40$ & 27.03 & 438 & 168.4 & 13.4 & 8.6 \\
\hline $7 / 18 / 1999$ & $12: 30: 40$ & 27.46 & 435 & 177.7 & 14.0 & 8.7 \\
\hline 7/18/1999 & 13:00:40 & 27.84 & 433 & 185.6 & 14.6 & 8.7 \\
\hline 7/18/1999 & $13: 30: 40$ & 28.27 & 429 & 192.9 & $15.0^{\mathbf{a}}$ & 8.8 \\
\hline 7/18/1999 & 14:00:40 & 28.37 & 428 & 188.5 & 14.6 & 8.8 \\
\hline $7 / 18 / 1999$ & $14: 30: 40$ & 28.49 & 428 & 186.9 & 14.5 & 8.8 \\
\hline 7/18/1999 & 15:00:40 & 28.45 & 429 & 176.6 & 13.7 & 8.7 \\
\hline 7/18/1999 & $15: 30: 40$ & 28.21 & 431 & 162.4 & 12.7 & 8.7 \\
\hline 7/18/1999 & $16: 00: 40$ & 28.03 & 432 & 153.9 & 12.0 & 8.7 \\
\hline 7/18/1999 & $16: 30: 40$ & 27.74 & 436 & 137.1 & 10.8 & 8.6 \\
\hline 7/18/1999 & $17: 00: 40$ & 27.52 & 439 & 128.6 & 10.1 & 8.5 \\
\hline 7/18/1999 & $17: 30: 40$ & 27.40 & 439 & 128.2 & 10.1 & 8.5 \\
\hline 7/18/1999 & 18:00:40 & 27.32 & 439 & 128.0 & 10.1 & 8.5 \\
\hline
\end{tabular}


Appendix Continued

\begin{tabular}{|c|c|c|c|c|c|c|}
\hline Date & Time & $\begin{array}{c}\text { Water } \\
\text { temperature } \\
\text { ( } \mathrm{C} \text { C) }\end{array}$ & $\begin{array}{c}\text { SC } \\
(\mu \mathrm{S} / \mathrm{cm})\end{array}$ & $\begin{array}{c}\text { DOsat } \\
\text { (percent) }\end{array}$ & $\begin{array}{c}\text { DO } \\
\text { (mg/L) }\end{array}$ & $\begin{array}{c}\text { pH } \\
\text { (standard } \\
\text { units) }\end{array}$ \\
\hline $7 / 18 / 1999$ & $18: 30: 40$ & 27.27 & 440 & 129.6 & 10.3 & 8.5 \\
\hline 7/18/1999 & 19:00:40 & 27.12 & 441 & 125.3 & 10.0 & 8.5 \\
\hline $7 / 18 / 1999$ & $19: 30: 40$ & 26.95 & 442 & 117.1 & 9.3 & 8.4 \\
\hline 7/18/1999 & 20:00:40 & 26.77 & 443 & 109.2 & 8.7 & 8.4 \\
\hline 7/18/1999 & 20:30:40 & 26.60 & 445 & 100.1 & 8.0 & 8.3 \\
\hline 7/18/1999 & 21:00:40 & 26.45 & 447 & 89.3 & 7.2 & 8.2 \\
\hline 7/18/1999 & $21: 30: 40$ & 26.29 & 448 & 79.9 & 6.4 & 8.2 \\
\hline $7 / 18 / 1999$ & $22: 00: 40$ & 26.15 & 450 & 72.1 & 5.8 & 8.1 \\
\hline 7/18/1999 & $22: 30: 40$ & 26.00 & 451 & 64.7 & 5.2 & 8.0 \\
\hline 7/18/1999 & 23:00:40 & 25.84 & 452 & 58.4 & 4.8 & 8.0 \\
\hline 7/18/1999 & $23: 30: 40$ & 25.66 & 453 & 52.6 & 4.3 & 7.9 \\
\hline 7/19/1999 & 0:00:40 & 25.49 & 454 & 48.2 & 3.9 & 7.8 \\
\hline $7 / 19 / 1999$ & $0: 30: 40$ & 25.34 & 455 & 45.4 & 3.7 & 7.8 \\
\hline $7 / 19 / 1999$ & 1:00:40 & 25.21 & 455 & 43.7 & 3.6 & 7.8 \\
\hline $7 / 19 / 1999$ & $1: 30: 40$ & 25.10 & 455 & 42.9 & 3.5 & 7.8 \\
\hline $7 / 19 / 1999$ & 2:00:40 & 25.00 & 455 & 41.3 & 3.4 & 7.8 \\
\hline $7 / 19 / 1999$ & $2: 30: 40$ & 24.91 & 455 & 40.5 & 3.4 & 7.8 \\
\hline 7/19/1999 & 3:00:40 & 24.82 & 455 & 40.0 & 3.3 & 7.7 \\
\hline 7/19/1999 & $3: 30: 40$ & 24.74 & 455 & 38.8 & 3.2 & 7.7 \\
\hline $7 / 19 / 1999$ & 4:00:40 & 24.66 & 455 & 38.1 & 3.2 & 7.7 \\
\hline $7 / 19 / 1999$ & $4: 30: 40$ & 24.59 & 455 & 37.1 & 3.1 & 7.7 \\
\hline $7 / 19 / 1999$ & $5: 00: 40$ & 24.55 & 455 & 37.0 & 3.1 & 7.7 \\
\hline $7 / 19 / 1999$ & $5: 30: 40$ & 24.49 & 455 & 36.2 & 3.0 & 7.7 \\
\hline $7 / 19 / 1999$ & 6:00:40 & 24.42 & 455 & 35.0 & 2.9 & 7.7 \\
\hline 7/19/1999 & $6: 30: 40$ & 24.37 & 455 & 35.9 & 3.0 & 7.7 \\
\hline 7/19/1999 & 7:00:40 & 24.30 & 455 & 38.9 & 3.3 & 7.7 \\
\hline 7/19/1999 & 7:30:40 & 24.35 & 454 & 46.2 & 3.9 & 7.8 \\
\hline 7/19/1999 & 8:00:40 & 24.42 & 454 & 57.5 & 4.8 & 7.8 \\
\hline 7/19/1999 & $8: 30: 40$ & 24.56 & 453 & 70.4 & 5.9 & 7.9 \\
\hline $7 / 19 / 1999$ & 9:00:40 & 24.76 & 451 & 84.0 & 7.0 & 8.0 \\
\hline $7 / 19 / 1999$ & $9: 30: 40$ & 25.02 & 450 & 99.6 & 8.2 & 8.1 \\
\hline $7 / 19 / 1999$ & 10:00:40 & 25.35 & 447 & 114.2 & 9.4 & 8.2 \\
\hline $7 / 19 / 1999$ & $10: 30: 40$ & 25.71 & 445 & 128.2 & 10.4 & 8.3 \\
\hline 7/19/1999 & 11:00:40 & 26.11 & 442 & 141.8 & 11.5 & 8.4 \\
\hline $7 / 19 / 1999$ & 11:30:40 & 26.52 & 440 & 153.3 & 12.3 & 8.5 \\
\hline $7 / 19 / 1999$ & $12: 00: 40$ & 26.90 & 437 & 162.1 & 12.9 & 8.6 \\
\hline 7/19/1999 & $12: 30: 40$ & 27.30 & 435 & 169.7 & 13.4 & 8.6 \\
\hline $7 / 19 / 1999$ & 13:00:40 & 27.49 & 434 & 171.0 & 13.5 & 8.6 \\
\hline $7 / 19 / 1999$ & $13: 30: 40$ & 27.77 & 433 & 176.0 & 13.8 & 8.7 \\
\hline $7 / 19 / 1999$ & 14:00:40 & 27.80 & 433 & 174.8 & 13.7 & 8.7 \\
\hline 7/19/1999 & $14: 30: 40$ & 27.77 & 432 & 172.8 & 13.6 & 8.7 \\
\hline 7/19/1999 & $15: 00: 40$ & 27.87 & 432 & 174.6 & 13.7 & 8.7 \\
\hline 7/19/1999 & $15: 30: 40$ & 27.72 & 429 & 166.8 & 13.1 & 8.7 \\
\hline 7/19/1999 & 16:00:40 & 27.71 & 430 & 169.0 & 13.3 & 8.7 \\
\hline $7 / 19 / 1999$ & $16: 30: 40$ & 27.63 & 433 & 160.6 & 12.6 & 8.7 \\
\hline $7 / 19 / 1999$ & 17:00:40 & 27.63 & 432 & 159.4 & 12.6 & 8.7 \\
\hline 7/19/1999 & $17: 30: 40$ & 27.70 & 429 & 163.0 & 12.8 & 8.7 \\
\hline 7/19/1999 & 18:00:40 & 27.60 & 432 & 156.4 & 12.3 & 8.7 \\
\hline 7/19/1999 & $18: 30: 40$ & 27.55 & 435 & 153.1 & 12.1 & 8.7 \\
\hline $7 / 19 / 1999$ & 19:00:40 & 27.34 & 436 & 140.3 & 11.1 & 8.6 \\
\hline 7/19/1999 & $19: 30: 40$ & 27.15 & 438 & 128.6 & 10.2 & 8.5 \\
\hline
\end{tabular}


Water-Quality Synoptic Sampling, July 1999: North Fork Shenandoah River, Virginia

Appendix Continued

\begin{tabular}{|c|c|c|c|c|c|c|}
\hline Date & Time & $\begin{array}{c}\text { Water } \\
\text { temperature } \\
\text { ('C) }\end{array}$ & $\begin{array}{c}\text { SC } \\
(\mu \mathbf{S} / \mathbf{c m})\end{array}$ & $\begin{array}{c}\text { DOsat } \\
\text { (percent) }\end{array}$ & $\begin{array}{c}\text { DO } \\
\text { (mg/L) }\end{array}$ & $\begin{array}{c}\text { pH } \\
\text { (standard } \\
\text { units) }\end{array}$ \\
\hline $7 / 19 / 1999$ & $20: 00: 40$ & 27.05 & 439 & 121.8 & 9.7 & 8.5 \\
\hline 7/19/1999 & $20: 30: 40$ & 26.91 & 441 & 109.2 & 8.7 & 8.4 \\
\hline $7 / 19 / 1999$ & 21:00:40 & 26.74 & 444 & 93.0 & 7.4 & 8.3 \\
\hline 7/19/1999 & $21: 30: 40$ & 26.58 & 447 & 83.8 & 6.7 & 8.2 \\
\hline $7 / 19 / 1999$ & 22:00:40 & 26.42 & 449 & 74.2 & 6.0 & 8.2 \\
\hline $7 / 19 / 1999$ & $22: 30: 40$ & 26.26 & 451 & 64.6 & 5.2 & 8.1 \\
\hline 7/19/1999 & $23: 00: 40$ & 26.10 & 452 & 57.8 & 4.7 & 8.0 \\
\hline $7 / 19 / 1999$ & $23: 30: 40$ & 25.93 & 453 & 51.2 & 4.2 & 7.9 \\
\hline $7 / 20 / 1999$ & 0:00:40 & 25.74 & 454 & 45.4 & 3.7 & 7.9 \\
\hline 7/20/1999 & $0: 30: 40$ & 25.56 & 455 & 40.2 & 3.3 & 7.8 \\
\hline $7 / 20 / 1999$ & 1:00:40 & 25.39 & 455 & 35.8 & 2.9 & 7.8 \\
\hline 7/20/1999 & 1:30:40 & 25.23 & 456 & 33.0 & 2.7 & 7.7 \\
\hline $7 / 20 / 1999$ & 2:00:40 & 25.10 & 456 & 31.4 & 2.6 & 7.7 \\
\hline $7 / 20 / 1999$ & $2: 30: 40$ & 24.97 & 457 & 31.5 & 2.6 & 7.7 \\
\hline $7 / 20 / 1999$ & 3:00:40 & 24.85 & 457 & 31.4 & 2.6 & 7.7 \\
\hline $7 / 20 / 1999$ & $3: 30: 40$ & 24.74 & 457 & 30.2 & 2.5 & 7.7 \\
\hline 7/20/1999 & 4:00:40 & 24.61 & 457 & 28.1 & 2.3 & 7.7 \\
\hline $7 / 20 / 1999$ & 4:30:40 & 24.53 & 457 & 28.1 & 2.3 & 7.7 \\
\hline 7/20/1999 & 5:00:40 & 24.44 & 457 & 27.6 & 2.3 & 7.6 \\
\hline $7 / 20 / 1999$ & $5: 30: 40$ & 24.37 & 457 & 26.6 & 2.2 & 7.6 \\
\hline 7/20/1999 & 6:00:40 & 24.32 & 457 & 26.2 & $2.2^{\mathbf{b}}$ & 7.6 \\
\hline $7 / 20 / 1999$ & $6: 30: 40$ & 24.30 & 457 & 27.4 & 2.3 & 7.6 \\
\hline $7 / 20 / 1999$ & 7:00:40 & 24.29 & 457 & 29.3 & 2.5 & 7.6 \\
\hline $7 / 20 / 1999$ & 7:30:40 & 24.32 & 457 & 30.5 & 2.6 & 7.7 \\
\hline $7 / 20 / 1999$ & 8:00:40 & 24.34 & 457 & 33.5 & 2.8 & 7.7 \\
\hline $7 / 20 / 1999$ & 8:30:40 & 24.37 & 457 & 36.7 & 3.1 & 7.7 \\
\hline 7/20/1999 & 9:00:40 & 24.46 & 457 & 48.2 & 4.0 & 7.7 \\
\hline $7 / 20 / 1999$ & $9: 30: 40$ & 24.62 & 456 & 62.9 & 5.2 & 7.8 \\
\hline $7 / 20 / 1999$ & 10:00:40 & 24.87 & 454 & 84.0 & 7.0 & 7.9 \\
\hline $7 / 20 / 1999$ & $10: 30: 40$ & 25.20 & 452 & 102.5 & 8.4 & 8.1 \\
\hline $7 / 20 / 1999$ & 11:00:40 & 25.37 & 449 & 112.1 & 9.2 & 8.2 \\
\hline 7/20/1999 & 11:30:40 & 25.60 & 448 & 124.4 & 10.2 & 8.3 \\
\hline 7/20/1999 & 12:00:40 & 25.98 & 444 & 145.8 & 11.8 & 8.4 \\
\hline
\end{tabular}

$\begin{array}{lllllll}7 / 15 / 1999 & 12: 30: 40 & 25.23 & - & 172.6 & 14.2 & 8.7 \\ 7 / 15 / 1999 & 13: 00: 40 & 25.68 & - & 177.6 & 14.5 & 8.7 \\ 7 / 15 / 1999 & 13: 30: 40 & 26.11 & - & 181.8 & 14.7 & 8.8 \\ 7 / 15 / 1999 & 14: 00: 40 & 26.47 & - & 186.4 & 15.0 & 8.8 \\ 7 / 15 / 1999 & 14: 30: 40 & 26.54 & - & 184.2 & 14.8 & 8.8 \\ 7 / 15 / 1999 & 15: 00: 40 & 26.78 & - & 185.3 & 14.8 & 8.8 \\ 7 / 15 / 1999 & 15: 30: 40 & 26.86 & - & 184.1 & 14.7 & 8.8 \\ 7 / 15 / 1999 & 16: 00: 40 & 26.81 & - & 181 & 14.5 & 8.8 \\ 7 / 15 / 1999 & 16: 30: 40 & 26.71 & - & 176.2 & 14.1 & 8.8 \\ 7 / 15 / 1999 & 17: 00: 40 & 26.34 & - & 162.5 & 13.1 & 8.7 \\ 7 / 15 / 1999 & 17: 30: 40 & 26.11 & - & 155.3 & 12.6 & 8.7 \\ 7 / 15 / 1999 & 18: 00: 40 & 25.79 & - & 140.5 & 11.5 & 8.6 \\ 7 / 15 / 1999 & 18: 30: 40 & 25.65 & - & 137.5 & 11.2 & 8.6 \\ 7 / 15 / 1999 & 19: 00: 40 & 25.46 & - & 129.3 & 10.6 & 8.5 \\ 7 / 15 / 1999 & 19: 30: 40 & 25.29 & - & 123.3 & 10.1 & 8.5 \\ 7 / 15 / 1999 & 20: 00: 40 & 25 & - & 113.5 & 9.4 & 8.4\end{array}$


Appendix Continued

\begin{tabular}{|c|c|c|c|c|c|c|}
\hline Date & Time & $\begin{array}{c}\text { Water } \\
\text { temperature } \\
\text { ('C) }\end{array}$ & $\begin{array}{c}\text { SC } \\
(\mu \mathbf{S} / \mathbf{c m})\end{array}$ & $\begin{array}{c}\text { DOsat } \\
\text { (percent) }\end{array}$ & $\begin{array}{c}\text { DO } \\
\text { (mg/L) }\end{array}$ & $\begin{array}{c}\text { pH } \\
\text { (standard } \\
\text { units) }\end{array}$ \\
\hline $7 / 15 / 1999$ & $20: 30: 40$ & 24.68 & - & 101.4 & 8.4 & 8.3 \\
\hline 7/15/1999 & 21:00:40 & 24.39 & - & 90.2 & 7.5 & 8.2 \\
\hline 7/15/1999 & $21: 30: 40$ & 24.14 & - & 82.8 & 7.0 & 8.1 \\
\hline $7 / 15 / 1999$ & 22:00:40 & 23.96 & - & 76.9 & 6.5 & 8.1 \\
\hline 7/15/1999 & $22: 30: 40$ & 23.82 & - & 72.3 & 6.1 & 8.0 \\
\hline 7/15/1999 & 23:00:40 & 23.71 & - & 68.9 & 5.8 & 8.0 \\
\hline $7 / 15 / 1999$ & $23: 30: 40$ & 23.62 & - & 66.1 & 5.6 & 8.0 \\
\hline $7 / 16 / 1999$ & 0:00:40 & 23.56 & - & 64.4 & 5.5 & 7.9 \\
\hline $7 / 16 / 1999$ & $0: 30: 40$ & 23.49 & - & 63.2 & 5.4 & 7.9 \\
\hline 7/16/1999 & 1:00:40 & 23.43 & - & 62.4 & 5.3 & 7.9 \\
\hline 7/16/1999 & 1:30:40 & 23.37 & - & 61.5 & 5.2 & 7.9 \\
\hline $7 / 16 / 1999$ & 2:00:40 & 23.34 & - & 61 & 5.2 & 7.9 \\
\hline 7/16/1999 & $2: 30: 40$ & 23.29 & - & 60.3 & 5.1 & 7.9 \\
\hline $7 / 16 / 1999$ & 3:00:40 & 23.24 & - & 59.6 & 5.1 & 7.9 \\
\hline $7 / 16 / 1999$ & $3: 30: 40$ & 23.21 & - & 58.9 & 5.0 & 7.9 \\
\hline 7/16/1999 & 4:00:40 & 23.15 & - & 58.3 & 5.0 & 7.9 \\
\hline $7 / 16 / 1999$ & 4:30:40 & 23.09 & - & 57.2 & 4.9 & 7.9 \\
\hline $7 / 16 / 1999$ & 5:00:40 & 23.06 & - & 56.6 & 4.9 & 7.9 \\
\hline $7 / 16 / 1999$ & $5: 30: 40$ & 23 & - & 55.5 & 4.8 & 7.9 \\
\hline 7/16/1999 & 6:00:40 & 22.96 & - & 54.9 & 4.7 & 7.9 \\
\hline 7/16/1999 & $6: 30: 40$ & 22.91 & - & 55.2 & 4.7 & 7.9 \\
\hline $7 / 16 / 1999$ & 7:00:40 & 22.91 & - & 58.3 & 5.0 & 7.9 \\
\hline $7 / 16 / 1999$ & $7: 30: 40$ & 22.95 & - & 64.9 & 5.6 & 7.9 \\
\hline $7 / 16 / 1999$ & 8:00:40 & 23.06 & - & 75 & 6.4 & 8.0 \\
\hline 7/16/1999 & $8: 30: 40$ & 23.24 & - & 86.9 & 7.4 & 8.1 \\
\hline 7/16/1999 & 9:00:40 & 23.51 & - & 100.3 & 8.5 & 8.2 \\
\hline 7/16/1999 & 9:30:40 & 23.83 & - & 114 & 9.6 & 8.3 \\
\hline $7 / 16 / 1999$ & 10:00:40 & 24.2 & - & 127.4 & 10.7 & 8.4 \\
\hline $7 / 16 / 1999$ & $10: 30: 40$ & 24.63 & - & 140 & 11.6 & 8.5 \\
\hline $7 / 16 / 1999$ & 11:00:40 & 25.1 & - & 151.4 & 12.5 & 8.6 \\
\hline $7 / 16 / 1999$ & $11: 30: 40$ & 25.54 & - & 160.5 & 13.1 & 8.6 \\
\hline $7 / 16 / 1999$ & $12: 00: 40$ & 26 & - & 168.6 & 13.7 & 8.7 \\
\hline $7 / 16 / 1999$ & $12: 30: 40$ & 26.38 & - & 173.6 & 14.0 & 8.7 \\
\hline 7/16/1999 & 13:00:40 & 26.91 & - & 182 & 14.5 & 8.8 \\
\hline 7/16/1999 & $13: 30: 40$ & 27.11 & - & 181.6 & 14.4 & 8.8 \\
\hline $7 / 16 / 1999$ & $14: 00: 40$ & 27.21 & - & 179 & 14.2 & 8.8 \\
\hline 7/16/1999 & $14: 30: 40$ & 27.53 & - & 182.9 & 14.4 & 8.8 \\
\hline 7/16/1999 & $15: 00: 40$ & 27.78 & - & 185.9 & 14.6 & 8.8 \\
\hline 7/16/1999 & $15: 30: 40$ & 27.84 & - & 185.3 & 14.5 & 8.8 \\
\hline $7 / 16 / 1999$ & $16: 00: 40$ & 28.09 & - & 189 & 14.8 & 8.8 \\
\hline 7/16/1999 & $16: 30: 40$ & 27.87 & - & 179.9 & 14.1 & 8.8 \\
\hline $7 / 16 / 1999$ & $17: 00: 40$ & 27.8 & - & 177.1 & 13.9 & 8.8 \\
\hline $7 / 16 / 1999$ & $17: 30: 40$ & 27.63 & - & 170.3 & 13.4 & 8.8 \\
\hline 7/16/1999 & 18:00:40 & 27.49 & - & 165.5 & 13.1 & 8.7 \\
\hline 7/16/1999 & $18: 30: 40$ & 27.24 & - & 155.9 & 12.4 & 8.7 \\
\hline 7/16/1999 & 19:00:40 & 26.97 & - & 144.9 & 11.6 & 8.6 \\
\hline $7 / 16 / 1999$ & $19: 30: 40$ & 26.7 & - & 132.5 & 10.6 & 8.6 \\
\hline 7/16/1999 & 20:00:40 & 26.42 & - & 119.9 & 9.7 & 8.5 \\
\hline 7/16/1999 & $20: 30: 40$ & 26.07 & - & 104.8 & 8.5 & 8.4 \\
\hline 7/16/1999 & 21:00:40 & 25.76 & - & 92.4 & 7.5 & 8.3 \\
\hline
\end{tabular}


Water-Quality Synoptic Sampling, July 1999: North Fork Shenandoah River, Virginia

Appendix Continued

\begin{tabular}{|c|c|c|c|c|c|c|}
\hline Date & Time & $\begin{array}{c}\text { Water } \\
\text { temperature } \\
\text { ('C) }\end{array}$ & $\begin{array}{c}\text { SC } \\
(\mu \mathbf{S} / \mathbf{c m})\end{array}$ & $\begin{array}{c}\text { DOsat } \\
\text { (percent) }\end{array}$ & $\begin{array}{c}\text { DO } \\
\text { (mg/L) }\end{array}$ & $\begin{array}{c}\text { pH } \\
\text { (standard } \\
\text { units) }\end{array}$ \\
\hline $7 / 16 / 1999$ & $21: 30: 40$ & 25.5 & - & 82.4 & 6.7 & 8.2 \\
\hline $7 / 16 / 1999$ & 22:00:40 & 25.29 & - & 74.4 & 6.1 & 8.1 \\
\hline 7/16/1999 & $22: 30: 40$ & 25.13 & - & 68.6 & 5.7 & 8.0 \\
\hline 7/16/1999 & 23:00:40 & 24.99 & - & 63.7 & 5.3 & 8.0 \\
\hline 7/16/1999 & $23: 30: 40$ & 24.86 & - & 59.9 & 5.0 & 7.9 \\
\hline 7/17/1999 & 0:00:40 & 24.74 & - & 56.8 & 4.7 & 7.9 \\
\hline 7/17/1999 & $0: 30: 40$ & 24.62 & - & 55.2 & 4.6 & 7.9 \\
\hline 7/17/1999 & 1:00:40 & 24.53 & - & 53.9 & 4.5 & 7.9 \\
\hline $7 / 17 / 1999$ & $1: 30: 40$ & 24.43 & - & 52.9 & 4.4 & 7.9 \\
\hline 7/17/1999 & 2:00:40 & 24.35 & - & 52.4 & 4.4 & 7.8 \\
\hline 7/17/1999 & 2:30:40 & 24.27 & - & 52 & 4.4 & 7.8 \\
\hline 7/17/1999 & 3:00:40 & 24.22 & - & 51.5 & 4.3 & 7.8 \\
\hline 7/17/1999 & 3:30:40 & 24.13 & - & 50.9 & 4.3 & 7.8 \\
\hline 7/17/1999 & 4:00:40 & 24.08 & - & 50.4 & 4.2 & 7.8 \\
\hline 7/17/1999 & 4:30:40 & 24.01 & - & 49.5 & 4.2 & 7.8 \\
\hline 7/17/1999 & 5:00:40 & 23.94 & - & 48.9 & 4.1 & 7.8 \\
\hline 7/17/1999 & 5:30:40 & 23.86 & - & 48.6 & 4.1 & 7.8 \\
\hline 7/17/1999 & 6:00:40 & 23.8 & - & 48 & 4.1 & 7.8 \\
\hline 7/17/1999 & 6:30:40 & 23.73 & - & 48.6 & 4.1 & 7.8 \\
\hline 7/17/1999 & 7:00:40 & 23.71 & - & 52.3 & 4.4 & 7.8 \\
\hline 7/17/1999 & 7:30:40 & 23.76 & - & 59.8 & 5.1 & 7.9 \\
\hline 7/17/1999 & 8:00:40 & 23.89 & - & 70.5 & 5.9 & 8.0 \\
\hline 7/17/1999 & 8:30:40 & 24.09 & - & 82.9 & 7.0 & 8.1 \\
\hline 7/17/1999 & 9:00:40 & 24.35 & - & 96.8 & 8.1 & 8.2 \\
\hline 7/17/1999 & $9: 30: 40$ & 24.71 & - & 111.7 & 9.3 & 8.3 \\
\hline 7/17/1999 & 10:00:40 & 25.13 & - & 126.1 & 10.4 & 8.4 \\
\hline 7/17/1999 & $10: 30: 40$ & 25.6 & - & 140.6 & 11.5 & 8.5 \\
\hline 7/17/1999 & 11:00:40 & 26.05 & - & 152.1 & 12.3 & 8.6 \\
\hline 7/17/1999 & $11: 30: 40$ & 26.47 & - & 160.9 & 12.9 & 8.6 \\
\hline $7 / 17 / 1999$ & $12: 00: 40$ & 26.91 & - & 169.7 & 13.5 & 8.7 \\
\hline 7/17/1999 & $12: 30: 40$ & 27.32 & - & 175 & 13.9 & 8.7 \\
\hline $7 / 17 / 1999$ & 13:00:40 & 27.55 & - & 176 & 13.9 & 8.7 \\
\hline 7/17/1999 & $13: 30: 40$ & 28.09 & - & 185.2 & 14.5 & 8.8 \\
\hline 7/17/1999 & 14:00:40 & 28.27 & - & 184.8 & 14.4 & 8.8 \\
\hline 7/17/1999 & $14: 30: 40$ & 28.78 & - & 193.3 & 14.9 & 8.9 \\
\hline 7/17/1999 & $15: 00: 40$ & 28.86 & - & 189.3 & 14.6 & 8.9 \\
\hline $7 / 17 / 1999$ & $15: 30: 40$ & 29.06 & - & 192.6 & 14.8 & 8.9 \\
\hline 7/17/1999 & $16: 00: 40$ & 29.23 & - & 193.9 & 14.8 & 8.9 \\
\hline 7/17/1999 & $16: 30: 40$ & 29.38 & - & 193.9 & 14.8 & 8.9 \\
\hline 7/17/1999 & 17:00:40 & 29.45 & - & 191.3 & 14.6 & 8.9 \\
\hline 7/17/1999 & $17: 30: 40$ & 29.29 & - & 183.1 & 14.0 & 8.9 \\
\hline 7/17/1999 & 18:00:40 & 29.15 & - & 175.1 & 13.4 & 8.8 \\
\hline 7/17/1999 & $18: 30: 40$ & 28.72 & - & 160.2 & 12.4 & 8.8 \\
\hline $7 / 17 / 1999$ & 19:00:40 & 28.32 & - & 143.9 & 11.2 & 8.7 \\
\hline 7/17/1999 & $19: 30: 40$ & 27.94 & - & 126.3 & 9.9 & 8.6 \\
\hline 7/17/1999 & 20:00:40 & 27.62 & - & 110.5 & 8.7 & 8.5 \\
\hline 7/17/1999 & $20: 30: 40$ & 27.3 & - & 96.3 & 7.6 & 8.4 \\
\hline 7/17/1999 & 21:00:40 & 27 & - & 84.8 & 6.8 & 8.3 \\
\hline 7/17/1999 & $21: 30: 40$ & 26.71 & - & 76.5 & 6.1 & 8.2 \\
\hline 7/17/1999 & $22: 00: 40$ & 26.44 & - & 69 & 5.6 & 8.1 \\
\hline
\end{tabular}


Appendix Continued

\begin{tabular}{|c|c|c|c|c|c|c|}
\hline Date & Time & $\begin{array}{c}\text { Water } \\
\text { temperature } \\
\text { (ㄷ) }\end{array}$ & $\begin{array}{c}\text { SC } \\
(\mu \mathbf{S} / \mathbf{c m})\end{array}$ & $\begin{array}{c}\text { DOsat } \\
\text { (percent) }\end{array}$ & $\begin{array}{c}\text { DO } \\
\text { (mg/L) }\end{array}$ & $\begin{array}{c}\text { pH } \\
\text { (standard } \\
\text { units) }\end{array}$ \\
\hline $7 / 17 / 1999$ & $22: 30: 40$ & 26.19 & - & 62.5 & 5.1 & 8.0 \\
\hline 7/17/1999 & 23:00:40 & 26 & - & 57.5 & 4.7 & 7.9 \\
\hline $7 / 17 / 1999$ & $23: 30: 40$ & 25.84 & - & 53.8 & 4.4 & 7.9 \\
\hline 7/18/1999 & 0:00:40 & 25.7 & - & 51.1 & 4.2 & 7.9 \\
\hline 7/18/1999 & $0: 30: 40$ & 25.58 & - & 49.4 & 4.0 & 7.8 \\
\hline 7/18/1999 & 1:00:40 & 25.48 & - & 48.3 & 4.0 & 7.8 \\
\hline $7 / 18 / 1999$ & $1: 30: 40$ & 25.38 & - & 47 & 3.9 & 7.8 \\
\hline $7 / 18 / 1999$ & 2:00:40 & 25.28 & - & 46.3 & 3.8 & 7.8 \\
\hline 7/18/1999 & $2: 30: 40$ & 25.18 & - & 45.5 & 3.7 & 7.8 \\
\hline 7/18/1999 & 3:00:40 & 25.1 & - & 44.9 & 3.7 & 7.8 \\
\hline 7/18/1999 & $3: 30: 40$ & 25.02 & - & 44.4 & 3.7 & 7.8 \\
\hline 7/18/1999 & 4:00:40 & 24.95 & - & 43.7 & 3.6 & 7.8 \\
\hline 7/18/1999 & 4:30:40 & 24.87 & - & 42.9 & 3.6 & 7.8 \\
\hline $7 / 18 / 1999$ & 5:00:40 & 24.79 & - & 42.1 & 3.5 & 7.8 \\
\hline $7 / 18 / 1999$ & $5: 30: 40$ & 24.72 & - & 41.5 & 3.4 & 7.8 \\
\hline 7/18/1999 & 6:00:40 & 24.65 & - & 40.7 & 3.4 & 7.8 \\
\hline $7 / 18 / 1999$ & $6: 30: 40$ & 24.58 & - & 41 & 3.4 & 7.8 \\
\hline 7/18/1999 & 7:00:40 & 24.56 & - & 44.3 & 3.7 & 7.8 \\
\hline $7 / 18 / 1999$ & 7:30:40 & 24.59 & - & 51.4 & 4.3 & 7.8 \\
\hline 7/18/1999 & 8:00:40 & 24.68 & - & 62.3 & 5.2 & 7.9 \\
\hline 7/18/1999 & $8: 30: 40$ & 24.83 & - & 75.3 & 6.2 & 8.0 \\
\hline $7 / 18 / 1999$ & 9:00:40 & 25.03 & - & 90 & 7.4 & 8.1 \\
\hline 7/18/1999 & $9: 30: 40$ & 25.31 & - & 106.2 & 8.7 & 8.2 \\
\hline $7 / 18 / 1999$ & 10:00:40 & 25.64 & - & 122 & 10.0 & 8.3 \\
\hline 7/18/1999 & $10: 30: 40$ & 26.02 & - & 137.3 & 11.1 & 8.4 \\
\hline 7/18/1999 & 11:00:40 & 26.42 & - & 151.1 & 12.2 & 8.5 \\
\hline 7/18/1999 & $11: 30: 40$ & 26.88 & - & 164.6 & 13.1 & 8.6 \\
\hline 7/18/1999 & $12: 00: 40$ & 27.33 & - & 174 & 13.8 & 8.7 \\
\hline $7 / 18 / 1999$ & $12: 30: 40$ & 27.81 & - & 184.1 & 14.5 & 8.8 \\
\hline 7/18/1999 & 13:00:40 & 28.26 & - & 191.2 & 14.9 & 8.8 \\
\hline 7/18/1999 & $13: 30: 40$ & 28.73 & - & 198 & $15.3^{\mathbf{a}}$ & 8.9 \\
\hline $7 / 18 / 1999$ & 14:00:40 & 28.7 & - & 188.5 & 14.6 & 8.8 \\
\hline 7/18/1999 & $14: 30: 40$ & 28.8 & - & 185.4 & 14.3 & 8.8 \\
\hline 7/18/1999 & $15: 00: 40$ & 28.8 & - & 181.9 & 14.0 & 8.8 \\
\hline 7/18/1999 & $15: 30: 40$ & 28.36 & - & 157.6 & 12.3 & 8.7 \\
\hline $7 / 18 / 1999$ & $16: 00: 40$ & 28.17 & - & 150.5 & 11.7 & 8.7 \\
\hline 7/18/1999 & $16: 30: 40$ & 27.92 & - & 139.4 & 10.9 & 8.7 \\
\hline 7/18/1999 & $17: 00: 40$ & 27.67 & - & 131.7 & 10.4 & 8.6 \\
\hline 7/18/1999 & $17: 30: 40$ & 27.62 & - & 134.1 & 10.6 & 8.6 \\
\hline 7/18/1999 & 18:00:40 & 27.55 & - & 132.6 & 10.5 & 8.6 \\
\hline 7/18/1999 & 18:30:40 & 27.47 & - & 131.6 & 10.4 & 8.6 \\
\hline 7/18/1999 & 19:00:40 & 27.4 & - & 130.9 & 10.4 & 8.6 \\
\hline $7 / 18 / 1999$ & $19: 30: 40$ & 27.22 & - & 121.6 & 9.6 & 8.5 \\
\hline 7/18/1999 & 20:00:40 & 26.98 & - & 111.5 & 8.9 & 8.4 \\
\hline 7/18/1999 & $20: 30: 40$ & 26.77 & - & 100.7 & 8.1 & 8.3 \\
\hline 7/18/1999 & 21:00:40 & 26.55 & - & 89.3 & 7.2 & 8.3 \\
\hline $7 / 18 / 1999$ & $21: 30: 40$ & 26.35 & - & 79.7 & 6.4 & 8.2 \\
\hline 7/18/1999 & $22: 00: 40$ & 26.16 & - & 71.2 & 5.8 & 8.1 \\
\hline 7/18/1999 & $22: 30: 40$ & 25.99 & - & 64.4 & 5.2 & 8.0 \\
\hline 7/18/1999 & 23:00:40 & 25.82 & - & 59.6 & 4.9 & 8.0 \\
\hline
\end{tabular}


Water-Quality Synoptic Sampling, July 1999: North Fork Shenandoah River, Virginia

Appendix Continued

\begin{tabular}{|c|c|c|c|c|c|c|}
\hline Date & Time & $\begin{array}{c}\text { Water } \\
\text { temperature } \\
\text { ('C) }\end{array}$ & $\begin{array}{c}\text { SC } \\
(\mu \mathrm{S} / \mathrm{cm})\end{array}$ & $\begin{array}{c}\text { D0sat } \\
\text { (percent) }\end{array}$ & $\begin{array}{c}\text { DO } \\
\text { (mg/L) }\end{array}$ & $\begin{array}{c}\text { pH } \\
\text { (standard } \\
\text { units) }\end{array}$ \\
\hline $7 / 18 / 1999$ & $23: 30: 40$ & 25.64 & - & 54.9 & 4.5 & 7.9 \\
\hline $7 / 19 / 1999$ & 0:00:40 & 25.47 & - & 51 & 4.2 & 7.9 \\
\hline $7 / 19 / 1999$ & 0:30:40 & 25.29 & - & 48 & 3.9 & 7.8 \\
\hline 7/19/1999 & 1:00:40 & 25.15 & - & 46.3 & 3.8 & 7.8 \\
\hline $7 / 19 / 1999$ & $1: 30: 40$ & 25.02 & - & 45.3 & 3.7 & 7.8 \\
\hline $7 / 19 / 1999$ & 2:00:40 & 24.91 & - & 44.6 & 3.7 & 7.8 \\
\hline $7 / 19 / 1999$ & $2: 30: 40$ & 24.83 & - & 44.4 & 3.7 & 7.8 \\
\hline $7 / 19 / 1999$ & 3:00:40 & 24.75 & - & 43.9 & 3.6 & 7.8 \\
\hline $7 / 19 / 1999$ & $3: 30: 40$ & 24.66 & - & 43.3 & 3.6 & 7.7 \\
\hline $7 / 19 / 1999$ & 4:00:40 & 24.6 & - & 43 & 3.6 & 7.7 \\
\hline $7 / 19 / 1999$ & 4:30:40 & 24.53 & - & 42.5 & 3.5 & 7.7 \\
\hline $7 / 19 / 1999$ & 5:00:40 & 24.47 & - & 42.1 & 3.5 & 7.7 \\
\hline $7 / 19 / 1999$ & $5: 30: 40$ & 24.4 & - & 41.5 & 3.5 & 7.7 \\
\hline $7 / 19 / 1999$ & 6:00:40 & 24.33 & - & 41 & 3.4 & 7.7 \\
\hline $7 / 19 / 1999$ & $6: 30: 40$ & 24.27 & - & 41.7 & 3.5 & 7.7 \\
\hline $7 / 19 / 1999$ & 7:00:40 & 24.25 & - & 45.6 & 3.8 & 7.8 \\
\hline $7 / 19 / 1999$ & 7:30:40 & 24.27 & - & 52.5 & 4.4 & 7.8 \\
\hline $7 / 19 / 1999$ & 8:00:40 & 24.36 & - & 63.4 & 5.3 & 7.9 \\
\hline $7 / 19 / 1999$ & $8: 30: 40$ & 24.52 & - & 76.7 & 6.4 & 8.0 \\
\hline $7 / 19 / 1999$ & 9:00:40 & 24.76 & - & 91.6 & 7.6 & 8.1 \\
\hline $7 / 19 / 1999$ & 9:30:40 & 25.08 & - & 107.3 & 8.9 & 8.2 \\
\hline $7 / 19 / 1999$ & 10:00:40 & 25.47 & - & 123.4 & 10.1 & 8.3 \\
\hline $7 / 19 / 1999$ & $10: 30: 40$ & 25.9 & - & 138.5 & 11.2 & 8.4 \\
\hline $7 / 19 / 1999$ & 11:00:40 & 26.35 & - & 152 & 12.3 & 8.5 \\
\hline $7 / 19 / 1999$ & $11: 30: 40$ & 26.83 & - & 164.4 & 13.1 & 8.6 \\
\hline $7 / 19 / 1999$ & 12:00:40 & 27.13 & - & 169.2 & 13.4 & 8.7 \\
\hline $7 / 19 / 1999$ & $12: 30: 40$ & 27.54 & - & 175.8 & 13.9 & 8.7 \\
\hline $7 / 19 / 1999$ & $13: 00: 40$ & 27.77 & - & 175.6 & 13.8 & 8.7 \\
\hline $7 / 19 / 1999$ & $13: 30: 40$ & 28.06 & - & 180.3 & 14.1 & 8.8 \\
\hline $7 / 19 / 1999$ & $14: 00: 40$ & 28.17 & - & 179.8 & 14.0 & 8.8 \\
\hline $7 / 19 / 1999$ & $14: 30: 40$ & 28.14 & - & 177.7 & 13.9 & 8.8 \\
\hline $7 / 19 / 1999$ & $15: 00: 40$ & 28.24 & - & 180.6 & 14.1 & 8.8 \\
\hline $7 / 19 / 1999$ & $15: 30: 40$ & 27.97 & - & 168.9 & 13.2 & 8.8 \\
\hline $7 / 19 / 1999$ & $16: 00: 40$ & 27.97 & - & 169.8 & 13.3 & 8.8 \\
\hline $7 / 19 / 1999$ & $16: 30: 40$ & 28.02 & - & 169.6 & 13.3 & 8.8 \\
\hline $7 / 19 / 1999$ & 17:00:40 & 28 & - & 167.1 & 13.1 & 8.8 \\
\hline $7 / 19 / 1999$ & $17: 30: 40$ & 28.04 & - & 167.8 & 13.1 & 8.8 \\
\hline $7 / 19 / 1999$ & 18:00:40 & 27.83 & - & 156.5 & 12.3 & 8.7 \\
\hline $7 / 19 / 1999$ & $18: 30: 40$ & 27.83 & - & 157 & 12.3 & 8.7 \\
\hline $7 / 19 / 1999$ & 19:00:40 & 27.66 & - & 149.2 & 11.7 & 8.7 \\
\hline $7 / 19 / 1999$ & $19: 30: 40$ & 27.39 & - & 132.6 & 10.5 & 8.6 \\
\hline $7 / 19 / 1999$ & 20:00:40 & 27.21 & - & 122 & 9.7 & 8.6 \\
\hline $7 / 19 / 1999$ & $20: 30: 40$ & 27.05 & - & 112.5 & 9.0 & 8.5 \\
\hline $7 / 19 / 1999$ & 21:00:40 & 26.85 & - & 98.6 & 7.9 & 8.4 \\
\hline $7 / 19 / 1999$ & 21:30:40 & 26.65 & - & 86.3 & 6.9 & 8.3 \\
\hline $7 / 19 / 1999$ & 22:00:40 & 26.46 & - & 76.1 & 6.1 & 8.2 \\
\hline $7 / 19 / 1999$ & $22: 30: 40$ & 26.27 & - & 67.4 & 5.4 & 8.1 \\
\hline $7 / 19 / 1999$ & $23: 00: 40$ & 26.09 & - & 60.5 & 4.9 & 8.0 \\
\hline $7 / 19 / 1999$ & $23: 30: 40$ & 25.92 & - & 55.1 & 4.5 & 7.9 \\
\hline $7 / 20 / 1999$ & 0:00:40 & 25.74 & - & 50.3 & 4.1 & 7.9 \\
\hline
\end{tabular}


Appendix Continued

\begin{tabular}{|c|c|c|c|c|c|c|}
\hline Date & Time & $\begin{array}{c}\text { Water } \\
\text { temperature } \\
\text { ('C) }\end{array}$ & $\begin{array}{c}\text { SC } \\
(\mu \mathbf{S} / \mathbf{c m})\end{array}$ & $\begin{array}{c}\text { DOsat } \\
\text { (percent) }\end{array}$ & $\begin{array}{c}\text { DO } \\
\text { (mg/L) }\end{array}$ & $\begin{array}{c}\text { pH } \\
\text { (standard } \\
\text { units) }\end{array}$ \\
\hline 7/20/1999 & $0: 30: 40$ & 25.57 & - & 46 & 3.8 & 7.8 \\
\hline 7/20/1999 & 1:00:40 & 25.39 & - & 42.8 & 3.5 & 7.8 \\
\hline 7/20/1999 & $1: 30: 40$ & 25.22 & - & 40.4 & 3.3 & 7.8 \\
\hline 7/20/1999 & 2:00:40 & 25.07 & - & 38.9 & 3.2 & 7.7 \\
\hline $7 / 20 / 1999$ & 2:30:40 & 24.93 & - & 37.9 & 3.1 & 7.7 \\
\hline 7/20/1999 & 3:00:40 & 24.79 & - & 37.8 & 3.1 & 7.7 \\
\hline 7/20/1999 & 3:30:40 & 24.69 & - & 37.2 & 3.1 & 7.7 \\
\hline 7/20/1999 & 4:00:40 & 24.58 & - & 37.4 & 3.1 & 7.7 \\
\hline $7 / 20 / 1999$ & 4:30:40 & 24.49 & - & 37.3 & 3.1 & 7.7 \\
\hline 7/20/1999 & 5:00:40 & 24.41 & - & 37.3 & 3.1 & 7.7 \\
\hline 7/20/1999 & $5: 30: 40$ & 24.34 & - & 36.8 & 3.1 & 7.7 \\
\hline $7 / 20 / 1999$ & 6:00:40 & 24.29 & - & 36.8 & $3.1^{\mathrm{b}}$ & 7.7 \\
\hline 7/20/1999 & $6: 30: 40$ & 24.28 & - & 36.9 & 3.1 & 7.7 \\
\hline 7/20/1999 & 7:00:40 & 24.27 & - & 38.3 & 3.2 & 7.7 \\
\hline 7/20/1999 & 7:30:40 & 24.26 & - & 38.4 & 3.2 & 7.7 \\
\hline $7 / 20 / 1999$ & 8:00:40 & 24.28 & - & 40 & 3.4 & 7.7 \\
\hline $7 / 20 / 1999$ & $8: 30: 40$ & 24.3 & - & 44.4 & 3.7 & 7.7 \\
\hline $7 / 20 / 1999$ & 9:00:40 & 24.43 & - & 58.3 & 4.9 & 7.8 \\
\hline 7/20/1999 & 9:30:40 & 24.61 & - & 71.3 & 5.9 & 7.9 \\
\hline $7 / 20 / 1999$ & 10:00:40 & 24.89 & - & 91.3 & 7.6 & 8.0 \\
\hline 7/20/1999 & 10:30:40 & 25.31 & - & 112.9 & 9.3 & 8.2 \\
\hline 7/20/1999 & 11:00:40 & 25.48 & - & 119.1 & 9.7 & 8.3 \\
\hline 7/20/1999 & 11:30:40 & 25.74 & - & 131.2 & 10.7 & 8.4 \\
\hline \multicolumn{7}{|c|}{ Site 22} \\
\hline 7/28/1999 & $14: 30: 40$ & 25.69 & 453 & 62.2 & 5.1 & 7.8 \\
\hline $7 / 28 / 1999$ & $15: 00: 40$ & 25.64 & 453 & 53.9 & 4.4 & 7.8 \\
\hline $7 / 28 / 1999$ & $15: 30: 40$ & 26.14 & 453 & 62.7 & 5.1 & 7.9 \\
\hline $7 / 28 / 1999$ & $16: 00: 40$ & 26.39 & 455 & 78.4 & 6.3 & 8.0 \\
\hline $7 / 28 / 1999$ & $16: 30: 40$ & 26.43 & 455 & 79.6 & 6.4 & 8.0 \\
\hline $7 / 28 / 1999$ & 17:00:40 & 25.71 & 454 & 53.7 & 4.4 & 7.8 \\
\hline $7 / 28 / 1999$ & $17: 30: 40$ & 25.72 & 423 & 79.7 & $6.5^{\mathrm{a}}$ & 8.0 \\
\hline $7 / 28 / 1999$ & 18:00:40 & 25.41 & 409 & 75.5 & 6.2 & 7.9 \\
\hline $7 / 28 / 1999$ & 18:30:40 & 25.49 & 416 & 71.7 & 5.9 & 7.9 \\
\hline $7 / 28 / 1999$ & 19:00:40 & 25.6 & 426 & 67 & 5.5 & 7.9 \\
\hline $7 / 28 / 1999$ & $19: 30: 40$ & 25.67 & 434 & 67.5 & 5.5 & 7.9 \\
\hline $7 / 28 / 1999$ & 20:00:40 & 25.42 & 430 & 48.7 & 4.0 & 7.8 \\
\hline $7 / 28 / 1999$ & $20: 30: 40$ & 25.32 & 428 & 41 & 3.4 & 7.7 \\
\hline $7 / 28 / 1999$ & 21:00:40 & 25.29 & 430 & 44.2 & 3.6 & 7.7 \\
\hline $7 / 28 / 1999$ & 21:30:40 & 25.29 & 432 & 46.8 & 3.8 & 7.7 \\
\hline $7 / 28 / 1999$ & $22: 00: 40$ & 25.24 & 427 & 44.4 & 3.7 & 7.7 \\
\hline $7 / 28 / 1999$ & $22: 30: 40$ & 25.16 & 427 & 44.6 & 3.7 & 7.7 \\
\hline $7 / 28 / 1999$ & $23: 00: 40$ & 25.12 & 431 & 43.6 & 3.6 & 7.7 \\
\hline $7 / 28 / 1999$ & $23: 30: 40$ & 25.08 & 436 & 43.3 & 3.6 & 7.7 \\
\hline 7/29/1999 & 0:00:40 & 25.03 & 440 & 39.2 & 3.2 & 7.7 \\
\hline 7/29/1999 & $0: 30: 40$ & 24.98 & 441 & 41.5 & 3.4 & 7.7 \\
\hline $7 / 29 / 1999$ & 1:00:40 & 24.92 & 443 & 42.6 & 3.5 & 7.7 \\
\hline 7/29/1999 & $1: 30: 40$ & 24.84 & 444 & 44.2 & 3.7 & 7.7 \\
\hline $7 / 29 / 1999$ & 2:00:40 & 24.73 & 444 & 44 & 3.7 & 7.7 \\
\hline $7 / 29 / 1999$ & $2: 30: 40$ & 24.65 & 444 & 41.4 & 3.4 & 7.7 \\
\hline
\end{tabular}


Water-Quality Synoptic Sampling, July 1999: North Fork Shenandoah River, Virginia

Appendix Continued

\begin{tabular}{|c|c|c|c|c|c|c|}
\hline Date & Time & $\begin{array}{c}\text { Water } \\
\text { temperature } \\
\text { ('C) }\end{array}$ & $\begin{array}{c}\text { SC } \\
(\mu \mathbf{S} / \mathbf{c m})\end{array}$ & $\begin{array}{c}\text { DOsat } \\
\text { (percent) }\end{array}$ & $\begin{array}{c}\text { DO } \\
\text { (mg/L) }\end{array}$ & $\begin{array}{c}\mathrm{pH} \\
\text { (standard } \\
\text { units) }\end{array}$ \\
\hline $7 / 29 / 1999$ & $3: 00: 40$ & 24.58 & 444 & 42.3 & 3.5 & 7.7 \\
\hline $7 / 29 / 1999$ & 3:30:40 & 24.5 & 445 & 41.4 & 3.5 & 7.7 \\
\hline $7 / 29 / 1999$ & 4:00:40 & 24.44 & 444 & 40.4 & 3.4 & 7.7 \\
\hline $7 / 29 / 1999$ & 4:30:40 & 24.37 & 445 & 40.6 & 3.4 & 7.7 \\
\hline $7 / 29 / 1999$ & 5:00:40 & 24.31 & 445 & 38.1 & 3.2 & 7.7 \\
\hline $7 / 29 / 1999$ & 5:30:40 & 24.26 & 446 & 39.4 & 3.3 & 7.7 \\
\hline $7 / 29 / 1999$ & 6:00:40 & 24.23 & 445 & 38.9 & 3.3 & 7.7 \\
\hline $7 / 29 / 1999$ & $6: 30: 40$ & 24.18 & 446 & 36.6 & 3.1 & 7.7 \\
\hline $7 / 29 / 1999$ & 7:00:40 & 24.16 & 445 & 37.1 & 3.1 & 7.7 \\
\hline $7 / 29 / 1999$ & 7:30:40 & 24.15 & 445 & 35.7 & 3.0 & 7.7 \\
\hline $7 / 29 / 1999$ & 8:00:40 & 24.14 & 445 & 36 & 3.0 & 7.7 \\
\hline $7 / 29 / 1999$ & 8:30:40 & 24.14 & 445 & 37.6 & 3.2 & 7.6 \\
\hline $7 / 29 / 1999$ & 9:00:40 & 24.15 & 445 & 37.2 & 3.1 & 7.6 \\
\hline 7/29/1999 & 9:30:40 & 24.16 & 446 & 29.6 & $2.5^{\mathbf{b}}$ & 7.6 \\
\hline $7 / 29 / 1999$ & 10:00:40 & 24.22 & 445 & 36.4 & 3.1 & 7.6 \\
\hline $7 / 29 / 1999$ & $10: 30: 40$ & 24.23 & 445 & 33.9 & 2.8 & 7.6 \\
\hline $7 / 29 / 1999$ & 11:00:40 & 24.27 & 445 & 35.2 & 2.9 & 7.6 \\
\hline $7 / 29 / 1999$ & $11: 30: 40$ & 24.33 & 445 & 38.6 & 3.2 & 7.7 \\
\hline $7 / 29 / 1999$ & $12: 00: 40$ & 24.37 & 444 & 36.9 & 3.1 & 7.7 \\
\hline $7 / 29 / 1999$ & $12: 30: 40$ & 24.44 & 443 & 37.7 & 3.2 & 7.6 \\
\hline $7 / 29 / 1999$ & $13: 00: 40$ & 24.53 & 443 & 40.4 & 3.4 & 7.7 \\
\hline $7 / 29 / 1999$ & $13: 30: 40$ & 24.55 & 442 & 40.3 & 3.4 & 7.7 \\
\hline $7 / 29 / 1999$ & 14:00:40 & 24.62 & 441 & 46.9 & 3.9 & 7.7 \\
\hline $7 / 29 / 1999$ & $14: 30: 40$ & 24.6 & 440 & 46.6 & 3.9 & 7.7 \\
\hline $7 / 29 / 1999$ & $15: 00: 40$ & 24.74 & 440 & 43.6 & 3.6 & 7.7 \\
\hline $7 / 29 / 1999$ & $15: 30: 40$ & 24.69 & 439 & 50 & 4.2 & 7.7 \\
\hline $7 / 29 / 1999$ & $16: 00: 40$ & 24.8 & 439 & 51.1 & 4.2 & 7.7 \\
\hline $7 / 29 / 1999$ & $16: 30: 40$ & 24.79 & 438 & 53.3 & 4.4 & 7.7 \\
\hline $7 / 29 / 1999$ & $17: 00: 40$ & 24.82 & 439 & 56.2 & 4.7 & 7.7 \\
\hline $7 / 29 / 1999$ & $17: 30: 40$ & 24.84 & 438 & 55.4 & 4.6 & 7.7 \\
\hline $7 / 29 / 1999$ & 18:00:40 & 24.88 & 438 & 55.4 & 4.6 & 7.7 \\
\hline $7 / 29 / 1999$ & $18: 30: 40$ & 24.9 & 439 & 52 & 4.3 & 7.7 \\
\hline $7 / 29 / 1999$ & 19:00:40 & 26.07 & 442 & 56 & 4.5 & 8.0 \\
\hline 7/29/1999 & $19: 30: 40$ & 26.24 & 444 & 66.1 & 5.3 & 8.0 \\
\hline $7 / 29 / 1999$ & 20:00:40 & 26.17 & 444 & 62.2 & 5.0 & 8.0 \\
\hline $7 / 29 / 1999$ & $20: 30: 40$ & 26.22 & 444 & 64.9 & 5.2 & 7.9 \\
\hline $7 / 29 / 1999$ & 21:00:40 & 26.15 & 444 & 59 & 4.8 & 7.9 \\
\hline $7 / 29 / 1999$ & $21: 30: 40$ & 26.08 & 444 & 58.2 & 4.7 & 7.9 \\
\hline 7/29/1999 & $22: 00: 40$ & 26 & 444 & 56.9 & 4.6 & 7.8 \\
\hline $7 / 29 / 1999$ & $22: 30: 40$ & 25.92 & 445 & 51.7 & 4.2 & 7.8 \\
\hline $7 / 29 / 1999$ & $23: 00: 40$ & 25.8 & 445 & 47.7 & 3.9 & 7.8 \\
\hline $7 / 29 / 1999$ & $23: 30: 40$ & 25.72 & 445 & 52.1 & 4.2 & 7.8 \\
\hline $7 / 30 / 1999$ & 0:00:40 & 25.63 & 445 & 50.9 & 4.2 & 7.8 \\
\hline $7 / 30 / 1999$ & 0:30:40 & 25.53 & 445 & 52.4 & 4.3 & 7.8 \\
\hline $7 / 30 / 1999$ & 1:00:40 & 25.43 & 446 & 51.1 & 4.2 & 7.8 \\
\hline $7 / 30 / 1999$ & $1: 30: 40$ & 25.35 & 446 & 51.3 & 4.2 & 7.8 \\
\hline $7 / 30 / 1999$ & 2:00:40 & 25.26 & 445 & 64.4 & 5.3 & 7.8 \\
\hline $7 / 30 / 1999$ & 2:30:40 & 25.17 & 445 & 61.9 & 5.1 & 7.7 \\
\hline $7 / 30 / 1999$ & 3:00:40 & 25.09 & 445 & 63.9 & 5.3 & 7.7 \\
\hline $7 / 30 / 1999$ & 3:30:40 & 25.02 & 445 & 67.6 & 5.6 & 7.7 \\
\hline
\end{tabular}


Appendix Continued

\begin{tabular}{|c|c|c|c|c|c|c|}
\hline Date & Time & $\begin{array}{c}\text { Water } \\
\text { temperature } \\
\text { ('C) }\end{array}$ & $\begin{array}{c}\text { SC } \\
(\mu \mathbf{S} / \mathbf{c m})\end{array}$ & $\begin{array}{c}\text { DOsat } \\
\text { (percent) }\end{array}$ & $\begin{array}{c}\text { DO } \\
\text { (mg/L) }\end{array}$ & $\begin{array}{c}\mathrm{pH} \\
\text { (standard } \\
\text { units) }\end{array}$ \\
\hline $7 / 30 / 1999$ & 4:00:40 & 24.95 & 445 & 66.2 & 5.5 & 7.7 \\
\hline 7/30/1999 & 4:30:40 & 24.9 & 445 & 64.7 & 5.4 & 7.7 \\
\hline 7/30/1999 & 5:00:40 & 24.84 & 445 & 65.5 & 5.4 & 7.7 \\
\hline 7/30/1999 & $5: 30: 40$ & 24.79 & 445 & 49.1 & 4.1 & 7.7 \\
\hline $7 / 30 / 1999$ & 6:00:40 & 24.74 & 445 & 70.8 & 5.9 & 7.7 \\
\hline 7/30/1999 & $6: 30: 40$ & 24.69 & 445 & 65.9 & 5.5 & 7.7 \\
\hline $7 / 30 / 1999$ & 7:00:40 & 24.66 & 445 & 66.9 & 5.6 & 7.7 \\
\hline 7/30/1999 & 7:30:40 & 24.65 & 444 & 68.1 & 5.7 & 7.7 \\
\hline 7/30/1999 & 8:00:40 & 24.64 & 445 & 69.9 & 5.8 & 7.7 \\
\hline 7/30/1999 & $8: 30: 40$ & 24.67 & 444 & 69.3 & 5.8 & 7.7 \\
\hline 7/30/1999 & 9:00:40 & 23.82 & 3 & 93.7 & 7.9 & 8.0 \\
\hline \multicolumn{7}{|c|}{ Site 23} \\
\hline 7/28/1999 & 13:00:40 & 25.84 & 447 & 108.2 & 8.8 & 8.2 \\
\hline $7 / 28 / 1999$ & $13: 30: 40$ & 25.96 & 445 & 115.3 & 9.4 & 8.3 \\
\hline $7 / 28 / 1999$ & 14:00:40 & 26.15 & 444 & 121.1 & 9.8 & 8.3 \\
\hline 7/28/1999 & $14: 30: 40$ & 26.36 & 443 & 126.3 & 10.2 & 8.4 \\
\hline $7 / 28 / 1999$ & $15: 00: 40$ & 26.48 & 442 & 127.8 & $10.3^{\mathbf{a}}$ & 8.4 \\
\hline $7 / 28 / 1999$ & $15: 30: 40$ & 26.53 & 442 & 126.8 & 10.2 & 8.4 \\
\hline $7 / 28 / 1999$ & $16: 00: 40$ & 26.6 & 442 & 126 & 10.1 & 8.4 \\
\hline $7 / 28 / 1999$ & $16: 30: 40$ & 26.64 & 441 & 124.9 & 10.0 & 8.4 \\
\hline $7 / 28 / 1999$ & $17: 00: 40$ & 26.35 & 431 & 118.6 & 9.6 & 8.4 \\
\hline $7 / 28 / 1999$ & $17: 30: 40$ & 25.74 & 387 & 108.5 & 8.8 & 8.4 \\
\hline $7 / 28 / 1999$ & 18:00:40 & 25.91 & 409 & 105.3 & 8.6 & 8.4 \\
\hline $7 / 28 / 1999$ & 18:30:40 & 25.81 & 411 & 98.1 & 8.0 & 8.4 \\
\hline $7 / 28 / 1999$ & 19:00:40 & 25.66 & 409 & 92 & 7.5 & 8.3 \\
\hline $7 / 28 / 1999$ & $19: 30: 40$ & 25.53 & 415 & 87.2 & 7.1 & 8.2 \\
\hline $7 / 28 / 1999$ & 20:00:40 & 25.38 & 420 & 83.5 & 6.8 & 8.2 \\
\hline $7 / 28 / 1999$ & $20: 30: 40$ & 25.21 & 425 & 80.2 & 6.6 & 8.1 \\
\hline $7 / 28 / 1999$ & 21:00:40 & 25.02 & 430 & 76.5 & 6.3 & 8.1 \\
\hline $7 / 28 / 1999$ & 21:30:40 & 24.84 & 435 & 73.3 & 6.1 & 8.0 \\
\hline $7 / 28 / 1999$ & 22:00:40 & 24.71 & 438 & 71.1 & 5.9 & 8.0 \\
\hline $7 / 28 / 1999$ & $22: 30: 40$ & 24.61 & 440 & 69.5 & 5.8 & 8.0 \\
\hline $7 / 28 / 1999$ & $23: 00: 40$ & 24.54 & 440 & 67.7 & 5.6 & 8.0 \\
\hline $7 / 28 / 1999$ & $23: 30: 40$ & 24.44 & 437 & 66.2 & 5.5 & 8.0 \\
\hline 7/29/1999 & 0:00:40 & 24.37 & 435 & 64.5 & 5.4 & 8.0 \\
\hline $7 / 29 / 1999$ & $0: 30: 40$ & 24.3 & 432 & 63 & 5.3 & 8.0 \\
\hline $7 / 29 / 1999$ & 1:00:40 & 24.23 & 429 & 61.5 & 5.2 & 7.9 \\
\hline $7 / 29 / 1999$ & $1: 30: 40$ & 24.17 & 426 & 59.9 & 5.0 & 7.9 \\
\hline $7 / 29 / 1999$ & 2:00:40 & 24.09 & 424 & 58.9 & 4.9 & 7.9 \\
\hline $7 / 29 / 1999$ & $2: 30: 40$ & 24.03 & 422 & 57.6 & 4.9 & 7.9 \\
\hline $7 / 29 / 1999$ & 3:00:40 & 23.96 & 422 & 57.2 & 4.8 & 7.9 \\
\hline $7 / 29 / 1999$ & 3:30:40 & 23.9 & 422 & 56.3 & 4.8 & 7.9 \\
\hline $7 / 29 / 1999$ & 4:00:40 & 23.84 & 422 & 55.6 & 4.7 & 7.9 \\
\hline 7/29/1999 & 4:30:40 & 23.78 & 424 & 55.2 & 4.7 & 7.9 \\
\hline 7/29/1999 & 5:00:40 & 23.73 & 425 & 54.5 & 4.6 & 7.9 \\
\hline $7 / 29 / 1999$ & $5: 30: 40$ & 23.69 & 427 & 53.7 & 4.5 & 7.9 \\
\hline $7 / 29 / 1999$ & 6:00:40 & 23.64 & 429 & 53.2 & 4.5 & 7.9 \\
\hline 7/29/1999 & $6: 30: 40$ & 23.59 & 431 & 52.8 & 4.5 & 7.9 \\
\hline 7/29/1999 & 7:00:40 & 23.57 & 433 & 51.8 & 4.4 & 7.8 \\
\hline
\end{tabular}


Water-Quality Synoptic Sampling, July 1999: North Fork Shenandoah River, Virginia

Appendix Continued

\begin{tabular}{|c|c|c|c|c|c|c|}
\hline Date & Time & $\begin{array}{c}\text { Water } \\
\text { temperature } \\
\text { (C) }\end{array}$ & $\begin{array}{c}\text { SC } \\
(\mu \mathbf{S} / \mathbf{c m})\end{array}$ & $\begin{array}{c}\text { DOsat } \\
\text { (percent) }\end{array}$ & $\begin{array}{c}\text { DO } \\
\text { (mg/L) }\end{array}$ & $\begin{array}{c}\text { pH } \\
\text { (standard } \\
\text { units) }\end{array}$ \\
\hline $7 / 29 / 1999$ & $7: 30: 40$ & 23.51 & 433 & 52.6 & 4.5 & 7.8 \\
\hline 7/29/1999 & 8:00:40 & 23.52 & 435 & 52.9 & 4.5 & 7.8 \\
\hline 7/29/1999 & 8:30:40 & 23.56 & 437 & 53.5 & 4.5 & 7.8 \\
\hline $7 / 29 / 1999$ & 9:00:40 & 23.68 & 438 & 56.9 & 4.8 & 7.9 \\
\hline $7 / 29 / 1999$ & $9: 30: 40$ & 23.8 & 439 & 58.4 & 4.9 & 7.9 \\
\hline $7 / 29 / 1999$ & 10:00:40 & 24.02 & 439 & 63.9 & 5.4 & 7.9 \\
\hline $7 / 29 / 1999$ & $10: 30: 40$ & 24.19 & 440 & 70 & 5.9 & 7.9 \\
\hline 7/29/1999 & 11:00:40 & 24.39 & 439 & 76.4 & 6.4 & 8.0 \\
\hline $7 / 29 / 1999$ & 11:30:40 & 24.58 & 439 & 83.8 & 7.0 & 8.0 \\
\hline $7 / 29 / 1999$ & 12:00:40 & 24.79 & 438 & 91.3 & 7.6 & 8.1 \\
\hline $7 / 29 / 1999$ & $12: 30: 40$ & 25.03 & 437 & 100.5 & 8.3 & 8.2 \\
\hline $7 / 29 / 1999$ & 13:00:40 & 25.28 & 436 & 109.1 & 9.0 & 8.2 \\
\hline 7/29/1999 & $13: 30: 40$ & 25.51 & 435 & 114.9 & 9.4 & 8.3 \\
\hline $7 / 29 / 1999$ & 14:00:40 & 25.82 & 434 & 120.8 & 9.8 & 8.3 \\
\hline $7 / 29 / 1999$ & $14: 30: 40$ & 26.03 & 434 & 122.1 & 9.9 & 8.3 \\
\hline $7 / 29 / 1999$ & $15: 00: 40$ & 26.26 & 433 & 124.3 & 10.0 & 8.4 \\
\hline $7 / 29 / 1999$ & $15: 30: 40$ & 26.4 & 433 & 125 & 10.1 & 8.4 \\
\hline $7 / 29 / 1999$ & $16: 00: 40$ & 26.48 & 433 & 124.3 & 10.0 & 8.4 \\
\hline $7 / 29 / 1999$ & $16: 30: 40$ & 26.51 & 433 & 122.3 & 9.8 & 8.4 \\
\hline $7 / 29 / 1999$ & 17:00:40 & 26.46 & 434 & 118.9 & 9.6 & 8.4 \\
\hline $7 / 29 / 1999$ & $17: 30: 40$ & 26.4 & 434 & 114.4 & 9.2 & 8.3 \\
\hline $7 / 29 / 1999$ & 18:00:40 & 26.31 & 435 & 109.8 & 8.9 & 8.3 \\
\hline $7 / 29 / 1999$ & $18: 30: 40$ & 26.23 & 436 & 105.8 & 8.5 & 8.3 \\
\hline $7 / 29 / 1999$ & 19:00:40 & 26.15 & 437 & 102.8 & 8.3 & 8.2 \\
\hline $7 / 29 / 1999$ & $19: 30: 40$ & 26.06 & 437 & 100.2 & 8.1 & 8.2 \\
\hline $7 / 29 / 1999$ & 20:00:40 & 25.95 & 437 & 97.2 & 7.9 & 8.2 \\
\hline $7 / 29 / 1999$ & $20: 30: 40$ & 25.83 & 437 & 94.3 & 7.7 & 8.2 \\
\hline $7 / 29 / 1999$ & 21:00:40 & 25.69 & 437 & 90.9 & 7.4 & 8.2 \\
\hline $7 / 29 / 1999$ & $21: 30: 40$ & 25.53 & 437 & 87.1 & 7.1 & 8.1 \\
\hline $7 / 29 / 1999$ & $22: 00: 40$ & 25.36 & 436 & 83 & 6.8 & 8.1 \\
\hline $7 / 29 / 1999$ & $22: 30: 40$ & 25.18 & 436 & 78.4 & 6.5 & 8.0 \\
\hline 7/29/1999 & 23:00:40 & 24.99 & 436 & 74 & 6.1 & 8.0 \\
\hline $7 / 29 / 1999$ & $23: 30: 40$ & 24.82 & 436 & 70.1 & 5.8 & 8.0 \\
\hline 7/30/1999 & 0:00:40 & 24.66 & 435 & 66.1 & 5.5 & 7.9 \\
\hline $7 / 30 / 1999$ & 0:30:40 & 24.5 & 435 & 62.8 & 5.2 & 7.9 \\
\hline 7/30/1999 & 1:00:40 & 24.4 & 435 & 59.9 & 5.0 & 7.9 \\
\hline $7 / 30 / 1999$ & $1: 30: 40$ & 24.3 & 434 & 58.1 & 4.9 & 7.9 \\
\hline $7 / 30 / 1999$ & 2:00:40 & 24.23 & 434 & 56.5 & 4.7 & 7.9 \\
\hline 7/30/1999 & 2:30:40 & 24.18 & 434 & 55.3 & 4.6 & 7.8 \\
\hline 7/30/1999 & 3:00:40 & 24.14 & 433 & 54.1 & 4.5 & 7.8 \\
\hline 7/30/1999 & $3: 30: 40$ & 24.1 & 433 & 53.6 & 4.5 & 7.8 \\
\hline $7 / 30 / 1999$ & 4:00:40 & 24.06 & 433 & 52.7 & 4.4 & 7.8 \\
\hline 7/30/1999 & 4:30:40 & 24.02 & 432 & 52.5 & 4.4 & 7.8 \\
\hline 7/30/1999 & 5:00:40 & 23.99 & 432 & 51.9 & 4.4 & 7.8 \\
\hline 7/30/1999 & $5: 30: 40$ & 23.95 & 433 & 51.6 & 4.3 & 7.8 \\
\hline 7/30/1999 & 6:00:40 & 23.91 & 432 & 51.3 & 4.3 & 7.8 \\
\hline 7/30/1999 & $6: 30: 40$ & 23.88 & 432 & 50.5 & $4.3^{\mathbf{b}}$ & 7.8 \\
\hline 7/30/1999 & 7:00:40 & 23.87 & 432 & 50.5 & 4.3 & 7.8 \\
\hline 7/30/1999 & 7:30:40 & 23.87 & 432 & 51 & 4.3 & 7.8 \\
\hline $7 / 30 / 1999$ & 8:00:40 & 23.84 & 431 & 51.1 & 4.3 & 7.8 \\
\hline
\end{tabular}


Appendix Continued

\begin{tabular}{|c|c|c|c|c|c|c|}
\hline Date & Time & $\begin{array}{c}\text { Water } \\
\text { temperature } \\
\text { (ㄷ) }\end{array}$ & $\begin{array}{c}\text { SC } \\
(\mu \mathrm{S} / \mathrm{cm})\end{array}$ & $\begin{array}{c}\text { D0sat } \\
\text { (percent) }\end{array}$ & $\begin{array}{c}\text { DO } \\
\text { (mg/L) }\end{array}$ & $\begin{array}{c}\text { pH } \\
\text { (standard } \\
\text { units) }\end{array}$ \\
\hline \multicolumn{7}{|c|}{ Site 27} \\
\hline $7 / 26 / 1999$ & $16: 30: 40$ & 26.77 & 366 & 188.2 & 15.0 & 8.6 \\
\hline $7 / 26 / 1999$ & 17:00:40 & 26.89 & 362 & 189.4 & 15.1 & 8.6 \\
\hline $7 / 26 / 1999$ & $17: 30: 40$ & 26.86 & 358 & 190.1 & 15.2 & 8.6 \\
\hline $7 / 26 / 1999$ & 18:00:40 & 26.78 & 354 & 196.1 & 15.7 & 8.6 \\
\hline $7 / 26 / 1999$ & $18: 30: 40$ & 26.59 & 352 & 190.3 & 15.3 & 8.6 \\
\hline $7 / 26 / 1999$ & 19:00:40 & 26.39 & 352 & 183.1 & 14.7 & 8.5 \\
\hline $7 / 26 / 1999$ & $19: 30: 40$ & 26.15 & 351 & 173.2 & 14.0 & 8.5 \\
\hline $7 / 26 / 1999$ & 20:00:40 & 25.87 & 351 & 161.1 & 13.1 & 8.4 \\
\hline $7 / 26 / 1999$ & $20: 30: 40$ & 25.6 & 354 & 148.7 & 12.1 & 8.4 \\
\hline $7 / 26 / 1999$ & 21:00:40 & 25.37 & 357 & 138.3 & 11.3 & 8.3 \\
\hline $7 / 26 / 1999$ & $21: 30: 40$ & 25.13 & 359 & 128 & 10.6 & 8.3 \\
\hline $7 / 26 / 1999$ & $22: 00: 40$ & 24.92 & 363 & 120.8 & 10.0 & 8.2 \\
\hline $7 / 26 / 1999$ & $22: 30: 40$ & 24.74 & 366 & 114.9 & 9.5 & 8.2 \\
\hline $7 / 26 / 1999$ & $23: 00: 40$ & 24.55 & 368 & 110.1 & 9.2 & 8.1 \\
\hline $7 / 26 / 1999$ & $23: 30: 40$ & 24.39 & 370 & 105.8 & 8.8 & 8.1 \\
\hline $7 / 27 / 1999$ & 0:00:40 & 24.23 & 373 & 102.1 & 8.6 & 8.1 \\
\hline 7/27/1999 & 0:30:40 & 24.05 & 376 & 99 & 8.3 & 8.0 \\
\hline 7/27/1999 & 1:00:40 & 23.89 & 379 & 95.3 & 8.0 & 8.0 \\
\hline 7/27/1999 & $1: 30: 40$ & 23.74 & 382 & 91.6 & 7.7 & 8.0 \\
\hline $7 / 27 / 1999$ & 2:00:40 & 23.59 & 385 & 88.2 & 7.5 & 7.9 \\
\hline $7 / 27 / 1999$ & $2: 30: 40$ & 23.45 & 387 & 84.2 & 7.2 & 7.9 \\
\hline 7/27/1999 & 3:00:40 & 23.31 & 390 & 80.3 & 6.8 & 7.9 \\
\hline 7/27/1999 & $3: 30: 40$ & 23.18 & 392 & 76.7 & 6.6 & 7.8 \\
\hline $7 / 27 / 1999$ & 4:00:40 & 23.05 & 393 & 73.6 & 6.3 & 7.8 \\
\hline $7 / 27 / 1999$ & $4: 30: 40$ & 22.94 & 394 & 70.5 & 6.1 & 7.8 \\
\hline 7/27/1999 & 5:00:40 & 22.84 & 394 & 67.4 & 5.8 & 7.7 \\
\hline 7/27/1999 & $5: 30: 40$ & 22.76 & 394 & 64.9 & 5.6 & 7.7 \\
\hline $7 / 27 / 1999$ & 6:00:40 & 22.71 & 394 & 62.3 & 5.4 & 7.7 \\
\hline $7 / 27 / 1999$ & $6: 30: 40$ & 22.68 & 393 & 59.9 & 5.2 & 7.7 \\
\hline $7 / 27 / 1999$ & 7:00:40 & 22.66 & 392 & 58.4 & 5.0 & 7.7 \\
\hline $7 / 27 / 1999$ & $7: 30: 40$ & 22.66 & 391 & 58.2 & 5.0 & 7.7 \\
\hline $7 / 27 / 1999$ & 8:00:40 & 22.68 & 390 & 57.7 & $5.0^{\mathbf{b}}$ & 7.7 \\
\hline 7/27/1999 & $8: 30: 40$ & 22.73 & 389 & 60.2 & 5.2 & 7.7 \\
\hline $7 / 27 / 1999$ & 9:00:40 & 22.8 & 388 & 65.3 & 5.6 & 7.7 \\
\hline $7 / 27 / 1999$ & 9:30:40 & 22.9 & 386 & 71.9 & 6.2 & 7.7 \\
\hline $7 / 27 / 1999$ & 10:00:40 & 23.04 & 385 & 79.1 & 6.8 & 7.8 \\
\hline $7 / 27 / 1999$ & 10:30:40 & 23.2 & 382 & 88.1 & 7.5 & 7.8 \\
\hline 7/27/1999 & 11:00:40 & 23.42 & 383 & 97.3 & 8.3 & 7.9 \\
\hline $7 / 27 / 1999$ & 11:30:40 & 23.61 & 380 & 105.9 & 9.0 & 8.0 \\
\hline 7/27/1999 & $12: 00: 40$ & 23.99 & 380 & 116.9 & 9.8 & 8.0 \\
\hline $7 / 27 / 1999$ & $12: 30: 40$ & 24.44 & 377 & 129 & 10.8 & 8.1 \\
\hline $7 / 27 / 1999$ & 13:00:40 & 24.95 & 377 & 140.8 & 11.6 & 8.2 \\
\hline 7/27/1999 & $13: 30: 40$ & 25.28 & 378 & 149 & 12.2 & 8.3 \\
\hline $7 / 27 / 1999$ & 14:00:40 & 25.59 & 376 & 156.7 & 12.8 & 8.3 \\
\hline 7/27/1999 & $14: 30: 40$ & 25.95 & 373 & 166.2 & 13.5 & 8.4 \\
\hline $7 / 27 / 1999$ & $15: 00: 40$ & 26.2 & 373 & 176.4 & 14.3 & 8.4 \\
\hline 7/27/1999 & $15: 30: 40$ & 26.31 & 372 & 181.4 & 14.6 & 8.5 \\
\hline 7/27/1999 & $16: 00: 40$ & 26.47 & 368 & 186.5 & 15.0 & 8.5 \\
\hline
\end{tabular}


Water-Quality Synoptic Sampling, July 1999: North Fork Shenandoah River, Virginia

Appendix Continued

\begin{tabular}{|c|c|c|c|c|c|c|}
\hline Date & Time & $\begin{array}{c}\text { Water } \\
\text { temperature } \\
\text { ('C) }\end{array}$ & $\begin{array}{c}\text { SC } \\
(\mu \mathbf{S} / \mathbf{c m})\end{array}$ & $\begin{array}{c}\text { DOsat } \\
\text { (percent) }\end{array}$ & $\begin{array}{c}\text { DO } \\
\text { (mg/L) }\end{array}$ & $\begin{array}{c}\text { pH } \\
\text { (standard } \\
\text { units) }\end{array}$ \\
\hline $7 / 27 / 1999$ & $16: 30: 40$ & 26.61 & 370 & 191 & 15.3 & 8.5 \\
\hline $7 / 27 / 1999$ & $17: 00: 40$ & 26.71 & 368 & 195.3 & 15.6 & 8.5 \\
\hline 7/27/1999 & $17: 30: 40$ & 26.83 & 366 & 198.5 & $15.9^{\mathbf{a}}$ & 8.6 \\
\hline $7 / 27 / 1999$ & 18:00:40 & 26.68 & 366 & 192.3 & 15.4 & 8.5 \\
\hline $7 / 27 / 1999$ & $18: 30: 40$ & 26.59 & 366 & 186 & 14.9 & 8.5 \\
\hline 7/27/1999 & 19:00:40 & 26.42 & 367 & 179.6 & 14.5 & 8.5 \\
\hline $7 / 27 / 1999$ & $19: 30: 40$ & 26.23 & 367 & 172.6 & 13.9 & 8.5 \\
\hline $7 / 27 / 1999$ & 20:00:40 & 26.03 & 368 & 163.5 & 13.2 & 8.4 \\
\hline $7 / 27 / 1999$ & $20: 30: 40$ & 25.83 & 370 & 152.5 & 12.4 & 8.4 \\
\hline $7 / 27 / 1999$ & 21:00:40 & 25.62 & 373 & 140.9 & 11.5 & 8.3 \\
\hline $7 / 27 / 1999$ & 21:30:40 & 25.4 & 375 & 130.1 & 10.7 & 8.2 \\
\hline $7 / 27 / 1999$ & $22: 00: 40$ & 25.21 & 377 & 122.3 & 10.1 & 8.2 \\
\hline $7 / 27 / 1999$ & $22: 30: 40$ & 25.03 & 380 & 115.7 & 9.6 & 8.1 \\
\hline $7 / 27 / 1999$ & 23:00:40 & 24.87 & 381 & 111.3 & 9.2 & 8.1 \\
\hline $7 / 27 / 1999$ & $23: 30: 40$ & 24.71 & 383 & 106.9 & 8.9 & 8.1 \\
\hline $7 / 28 / 1999$ & 0:00:40 & 24.56 & 384 & 103 & 8.6 & 8.0 \\
\hline $7 / 28 / 1999$ & 0:30:40 & 24.42 & 386 & 99.1 & 8.3 & 8.0 \\
\hline $7 / 28 / 1999$ & 1:00:40 & 24.28 & 387 & 95.4 & 8.0 & 8.0 \\
\hline $7 / 28 / 1999$ & $1: 30: 40$ & 24.12 & 389 & 92.3 & 7.7 & 7.9 \\
\hline $7 / 28 / 1999$ & 2:00:40 & 23.99 & 390 & 88.2 & 7.4 & 7.9 \\
\hline $7 / 28 / 1999$ & $2: 30: 40$ & 23.85 & 391 & 84.4 & 7.1 & 7.9 \\
\hline $7 / 28 / 1999$ & 3:00:40 & 23.7 & 393 & 81 & 6.9 & 7.8 \\
\hline 7/28/1999 & $3: 30: 40$ & 23.59 & 394 & 74.9 & 6.3 & 7.8 \\
\hline $7 / 28 / 1999$ & 4:00:40 & 23.46 & 394 & 73.7 & 6.3 & 7.8 \\
\hline $7 / 28 / 1999$ & 4:30:40 & 23.34 & 395 & 70.7 & 6.0 & 7.7 \\
\hline $7 / 28 / 1999$ & 5:00:40 & 23.24 & 395 & 67.7 & 5.8 & 7.7 \\
\hline $7 / 28 / 1999$ & $5: 30: 40$ & 23.15 & 395 & 64.9 & 5.5 & 7.7 \\
\hline $7 / 28 / 1999$ & 6:00:40 & 23.07 & 394 & 62.1 & 5.3 & 7.7 \\
\hline $7 / 28 / 1999$ & $6: 30: 40$ & 23.01 & 394 & 59.8 & 5.1 & 7.7 \\
\hline $7 / 28 / 1999$ & 7:00:40 & 22.97 & 393 & 58.8 & 5.0 & 7.6 \\
\hline 7/28/1999 & 7:30:40 & 22.97 & 392 & 61.1 & 5.2 & 7.7 \\
\hline \multicolumn{7}{|c|}{ Site 28} \\
\hline $7 / 26 / 1999$ & $16: 00: 40$ & 25.84 & 405 & 171.6 & 14.0 & 8.6 \\
\hline $7 / 26 / 1999$ & $16: 30: 40$ & 25.97 & 403 & 167.9 & 13.6 & 8.6 \\
\hline $7 / 26 / 1999$ & 17:00:40 & 26 & 400 & 165.9 & 13.4 & 8.7 \\
\hline $7 / 26 / 1999$ & $17: 30: 40$ & 25.96 & 396 & 163.3 & 13.2 & 8.6 \\
\hline $7 / 26 / 1999$ & 18:00:40 & 25.83 & 392 & 159.2 & 13.0 & 8.6 \\
\hline $7 / 26 / 1999$ & $18: 30: 40$ & 25.69 & 389 & 156.1 & 12.7 & 8.6 \\
\hline $7 / 26 / 1999$ & 19:00:40 & 25.53 & 386 & 151.7 & 12.4 & 8.6 \\
\hline $7 / 26 / 1999$ & $19: 30: 40$ & 25.42 & 384 & 145.2 & 11.9 & 8.5 \\
\hline $7 / 26 / 1999$ & $20: 00: 40$ & 25.31 & 382 & 138.3 & 11.4 & 8.5 \\
\hline $7 / 26 / 1999$ & $20: 30: 40$ & 25.14 & 382 & 129.4 & 10.7 & 8.4 \\
\hline $7 / 26 / 1999$ & 21:00:40 & 24.95 & 383 & 120.1 & 9.9 & 8.3 \\
\hline $7 / 26 / 1999$ & $21: 30: 40$ & 24.75 & 386 & 111.4 & 9.2 & 8.3 \\
\hline $7 / 26 / 1999$ & $22: 00: 40$ & 24.53 & 389 & 104.2 & 8.7 & 8.2 \\
\hline $7 / 26 / 1999$ & $22: 30: 40$ & 24.35 & 392 & 97.8 & 8.2 & 8.2 \\
\hline $7 / 26 / 1999$ & $23: 00: 40$ & 24.15 & 396 & 92.4 & 7.8 & 8.1 \\
\hline $7 / 26 / 1999$ & $23: 30: 40$ & 23.98 & 400 & 87.8 & 7.4 & 8.1 \\
\hline $7 / 27 / 1999$ & 0:00:40 & 23.81 & 404 & 84 & 7.1 & 8.0 \\
\hline
\end{tabular}


Appendix Continued

\begin{tabular}{|c|c|c|c|c|c|c|}
\hline Date & Time & $\begin{array}{c}\text { Water } \\
\text { temperature } \\
\text { ('C) }\end{array}$ & $\begin{array}{c}\text { SC } \\
(\mu \mathrm{S} / \mathbf{c m})\end{array}$ & $\begin{array}{c}\text { DOsat } \\
\text { (percent) }\end{array}$ & $\begin{array}{c}\text { DO } \\
\text { (mg/L) }\end{array}$ & $\begin{array}{c}\text { pH } \\
\text { (standard } \\
\text { units) }\end{array}$ \\
\hline $7 / 27 / 1999$ & $0: 30: 40$ & 23.65 & 407 & 80.7 & 6.8 & 8.0 \\
\hline $7 / 27 / 1999$ & 1:00:40 & 23.51 & 410 & 78 & 6.6 & 8.0 \\
\hline $7 / 27 / 1999$ & $1: 30: 40$ & 23.4 & 411 & 75.5 & 6.4 & 8.0 \\
\hline $7 / 27 / 1999$ & 2:00:40 & 23.26 & 414 & 73.5 & 6.3 & 7.9 \\
\hline 7/27/1999 & 2:30:40 & 23.14 & 415 & 71.3 & 6.1 & 7.9 \\
\hline 7/27/1999 & 3:00:40 & 23.01 & 417 & 69.1 & 5.9 & 7.9 \\
\hline 7/27/1999 & $3: 30: 40$ & 22.88 & 418 & 67 & 5.8 & 7.9 \\
\hline $7 / 27 / 1999$ & 4:00:40 & 22.77 & 418 & 64.7 & 5.6 & 7.9 \\
\hline $7 / 27 / 1999$ & 4:30:40 & 22.65 & 419 & 62.6 & 5.4 & 7.8 \\
\hline 7/27/1999 & 5:00:40 & 22.54 & 419 & 60.4 & 5.2 & 7.8 \\
\hline $7 / 27 / 1999$ & $5: 30: 40$ & 22.44 & 420 & 58.7 & 5.1 & 7.8 \\
\hline 7/27/1999 & 6:00:40 & 22.37 & 420 & 56.9 & 4.9 & 7.8 \\
\hline $7 / 27 / 1999$ & $6: 30: 40$ & 22.3 & 419 & 55.4 & 4.8 & 7.8 \\
\hline 7/27/1999 & 7:00:40 & 22.25 & 419 & 54.4 & 4.7 & 7.8 \\
\hline $7 / 27 / 1999$ & $7: 30: 40$ & 22.2 & 419 & 53.9 & $4.7^{\mathbf{b}}$ & 7.8 \\
\hline $7 / 27 / 1999$ & 8:00:40 & 22.18 & 418 & 54.2 & 4.7 & 7.7 \\
\hline $7 / 27 / 1999$ & $8: 30: 40$ & 22.19 & 418 & 58 & 5.1 & 7.8 \\
\hline 7/27/1999 & 9:00:40 & 22.23 & 403 & 63.8 & 5.6 & 7.8 \\
\hline $7 / 27 / 1999$ & 9:30:40 & 22.31 & 421 & 70.3 & 6.1 & 7.9 \\
\hline 7/27/1999 & 10:00:40 & 22.46 & 410 & 79 & 6.8 & 7.9 \\
\hline 7/27/1999 & $10: 30: 40$ & 22.61 & 405 & 88.6 & 7.7 & 8.0 \\
\hline $7 / 27 / 1999$ & 11:00:40 & 22.86 & 407 & 99.2 & 8.5 & 8.1 \\
\hline $7 / 27 / 1999$ & 11:30:40 & 23.08 & 411 & 109.9 & 9.4 & 8.2 \\
\hline $7 / 27 / 1999$ & $12: 00: 40$ & 23.45 & 411 & 121.8 & 10.4 & 8.3 \\
\hline 7/27/1999 & $12: 30: 40$ & 23.93 & 406 & 133.9 & 11.3 & 8.4 \\
\hline 7/27/1999 & 13:00:40 & 24.4 & 407 & 145.4 & 12.1 & 8.5 \\
\hline 7/27/1999 & $13: 30: 40$ & 24.78 & 402 & 153.9 & 12.8 & 8.6 \\
\hline 7/27/1999 & 14:00:40 & 25.04 & 404 & 160.1 & 13.2 & 8.6 \\
\hline $7 / 27 / 1999$ & $14: 30: 40$ & 25.51 & 402 & 167.1 & 13.7 & 8.7 \\
\hline $7 / 27 / 1999$ & $15: 00: 40$ & 25.88 & 400 & 174 & 14.1 & 8.7 \\
\hline 7/27/1999 & $15: 30: 40$ & 25.98 & 398 & 176.3 & 14.3 & 8.7 \\
\hline 7/27/1999 & $16: 00: 40$ & 26.05 & 397 & 174.5 & 14.1 & 8.7 \\
\hline 7/27/1999 & $16: 30: 40$ & 26.15 & 395 & 175.6 & 14.2 & 8.7 \\
\hline 7/27/1999 & 17:00:40 & 26.29 & 393 & 175.6 & 14.2 & 8.7 \\
\hline $7 / 27 / 1999$ & $17: 30: 40$ & 26.29 & 391 & 178.6 & $14.4^{\mathbf{a}}$ & 8.7 \\
\hline $7 / 27 / 1999$ & 18:00:40 & 26.19 & 390 & 173.2 & 14.0 & 8.6 \\
\hline $7 / 27 / 1999$ & 18:30:40 & 26.06 & 390 & 166.5 & 13.5 & 8.6 \\
\hline $7 / 27 / 1999$ & 19:00:40 & 25.92 & 389 & 159.1 & 12.9 & 8.6 \\
\hline 7/27/1999 & 19:30:40 & 25.77 & 396 & 153.1 & 12.5 & 8.5 \\
\hline $7 / 27 / 1999$ & 20:00:40 & 25.61 & 397 & 145.7 & 11.9 & 8.5 \\
\hline 7/27/1999 & $20: 30: 40$ & 25.43 & 398 & 136.6 & 11.2 & 8.4 \\
\hline 7/27/1999 & 21:00:40 & 25.25 & 399 & 124.5 & 10.2 & 8.3 \\
\hline $7 / 27 / 1999$ & 21:30:40 & 25.05 & 400 & 115.2 & 9.5 & 8.3 \\
\hline $7 / 27 / 1999$ & $22: 00: 40$ & 24.86 & 402 & 106.9 & 8.8 & 8.2 \\
\hline $7 / 27 / 1999$ & $22: 30: 40$ & 24.66 & 404 & 100 & 8.3 & 8.1 \\
\hline 7/27/1999 & $23: 00: 40$ & 24.47 & 406 & 94.2 & 7.9 & 8.1 \\
\hline 7/27/1999 & $23: 30: 40$ & 24.3 & 407 & 89.3 & 7.5 & 8.0 \\
\hline $7 / 28 / 1999$ & 0:00:40 & 24.15 & 408 & 85.2 & 7.2 & 8.0 \\
\hline 7/28/1999 & 0:30:40 & 24.01 & 410 & 82 & 6.9 & 8.0 \\
\hline $7 / 28 / 1999$ & 1:00:40 & 23.89 & 411 & 78.9 & 6.7 & 8.0 \\
\hline
\end{tabular}


Water-Quality Synoptic Sampling, July 1999: North Fork Shenandoah River, Virginia

Appendix Continued

\begin{tabular}{ccccccc}
\hline Date & Time & $\begin{array}{c}\text { Water } \\
\text { temperature } \\
(\mathbf{0} \text { ) }\end{array}$ & $\begin{array}{c}\text { SC } \\
(\mu \mathbf{S} / \mathbf{c m})\end{array}$ & $\begin{array}{c}\text { DOsat } \\
\text { (percent) }\end{array}$ & $\begin{array}{c}\text { Do } \\
(\mathbf{m g} / \mathbf{L})\end{array}$ & $\begin{array}{c}\text { pH } \\
\text { (standard } \\
\text { units) }\end{array}$ \\
\hline $7 / 28 / 1999$ & $1: 30: 40$ & 23.76 & 412 & 76.2 & 6.4 & 7.9 \\
$7 / 28 / 1999$ & $2: 00: 40$ & 23.65 & 413 & 73.9 & 6.3 & 7.9 \\
$7 / 28 / 1999$ & $2: 30: 40$ & 23.53 & 415 & 71.6 & 6.1 & 7.9 \\
$7 / 28 / 1999$ & $3: 00: 40$ & 23.41 & 416 & 69.3 & 5.9 & 7.9 \\
$7 / 28 / 1999$ & $3: 30: 40$ & 23.29 & 416 & 67.1 & 5.7 & 7.8 \\
$7 / 28 / 1999$ & $4: 00: 40$ & 23.17 & 418 & 64.9 & 5.6 & 7.8 \\
$7 / 28 / 1999$ & $4: 30: 40$ & 23.06 & 419 & 62.7 & 5.4 & 7.8 \\
$7 / 28 / 1999$ & $5: 00: 40$ & 22.96 & 419 & 60.4 & 5.2 & 7.8 \\
$7 / 28 / 1999$ & $5: 30: 40$ & 22.85 & 419 & 58.3 & 5.0 & 7.8 \\
$7 / 28 / 1999$ & $6: 00: 40$ & 22.74 & 420 & 56.6 & 4.9 & 7.8 \\
$7 / 28 / 1999$ & $6: 30: 40$ & 22.65 & 420 & 55.1 & 4.8 & 7.8 \\
$7 / 28 / 1999$ & $7: 00: 40$ & 22.57 & 420 & 55.1 & 4.8 & 7.7 \\
$7 / 28 / 1999$ & $7: 30: 40$ & 22.54 & 420 & 57.1 & 4.9 & 7.8 \\
\hline
\end{tabular}

$\begin{array}{lcccccc}7 / 26 / 1999 & 15: 00: 40 & 24.48 & 446 & 85.6 & 7.1 & 8.0 \\ 7 / 26 / 1999 & 15: 30: 40 & 24.73 & 447 & 88.6 & 7.4 & 8.0 \\ 7 / 26 / 1999 & 16: 00: 40 & 24.27 & 452 & 74.7 & 6.3 & 7.9 \\ 7 / 26 / 1999 & 16: 30: 40 & 24.52 & 449 & 92.4 & 7.7 & 8.0 \\ 7 / 26 / 1999 & 17: 00: 40 & 24.63 & 449 & 101.9 & 8.5 & 8.0 \\ 7 / 26 / 1999 & 17: 30: 40 & 23.77 & 445 & 88.1 & 7.4 & 8.0 \\ 7 / 26 / 1999 & 18: 00: 40 & 23.92 & 444 & 90.4 & 7.6 & 8.0 \\ 7 / 26 / 1999 & 18: 30: 40 & 23.79 & 443 & 105.1 & 8.9 & 8.2 \\ 7 / 26 / 1999 & 19: 00: 40 & 23.96 & 442 & 124 & 10.4 & 8.3 \\ 7 / 26 / 1999 & 19: 30: 40 & 24.06 & 441 & 131.6 & 11.1 & 8.4 \\ 7 / 26 / 1999 & 20: 00: 40 & 24.15 & 439 & 140.2 & 11.8 & 8.4 \\ 7 / 26 / 1999 & 20: 30: 40 & 24.25 & 438 & 144.3 & 12.1 & 8.5 \\ 7 / 26 / 1999 & 21: 00: 40 & 24.26 & 439 & 150.1 & 12.6 & 8.5 \\ 7 / 26 / 1999 & 21: 30: 40 & 24.57 & 431 & 151.9 & 12.6 & 8.5 \\ 7 / 26 / 1999 & 22: 00: 40 & 24.73 & 431 & 151.2 & 12.5 & 8.5 \\ 7 / 26 / 1999 & 22: 30: 40 & 24.84 & 431 & 146.6 & 12.1 & 8.5 \\ 7 / 26 / 1999 & 23: 00: 40 & 24.58 & 436 & 146.3 & 12.2 & 8.5 \\ 7 / 26 / 1999 & 23: 30: 40 & 24.61 & 438 & 144.6 & 12.0 & 8.5 \\ 7 / 27 / 1999 & 0: 00: 40 & 24.67 & 437 & 145.3 & 12.1 & 8.5 \\ 7 / 27 / 1999 & 0: 30: 40 & 24.65 & 437 & 143.7 & 11.9 & 8.5 \\ 7 / 27 / 1999 & 1: 00: 40 & 24.66 & 437 & 139.5 & 11.6 & 8.5 \\ 7 / 27 / 1999 & 1: 30: 40 & 24.63 & 438 & 137 & 11.4 & 8.5 \\ 7 / 27 / 1999 & 2: 00: 40 & 24.65 & 438 & 131.9 & 11.0 & 8.4 \\ 7 / 27 / 1999 & 2: 30: 40 & 24.63 & 437 & 129.4 & 10.8 & 8.4 \\ 7 / 27 / 1999 & 3: 00: 40 & 24.59 & 437 & 129.2 & 10.7 & 8.4 \\ 7 / 27 / 1999 & 3: 30: 40 & 24.48 & 438 & 125.3 & 10.5 & 8.4 \\ 7 / 27 / 1999 & 4: 00: 40 & 24.39 & 439 & 116.5 & 9.7 & 8.4 \\ 7 / 27 / 1999 & 4: 30: 40 & 24.27 & 439 & 111.7 & 9.4 & 8.3 \\ 7 / 27 / 1999 & 5: 00: 40 & 24.09 & 440 & 101.1 & 8.5 & 8.2 \\ 7 / 27 / 1999 & 5: 30: 40 & 23.97 & 439 & 92.4 & 7.8 & 8.2 \\ 7 / 27 / 1999 & 6: 00: 40 & 23.87 & 439 & 90.3 & 7.6 & 8.1 \\ 7 / 27 / 1999 & 6: 30: 40 & 23.8 & 441 & 87.4 & 7.4 & 8.1 \\ 7 / 27 / 1999 & 7: 00: 40 & 23.62 & 442 & 80.1 & 6.8 & 8.0 \\ 7 / 27 / 1999 & 7: 30: 40 & 23.52 & 444 & 74.9 & 6.4 & 8.0 \\ 7 / 27 / 1999 & 8: 00: 40 & 24.08 & 440 & 107 & 9.0 & 8.3\end{array}$


Appendix Continued

\begin{tabular}{|c|c|c|c|c|c|c|}
\hline Date & Time & $\begin{array}{c}\text { Water } \\
\text { temperature } \\
\text { ('C) }\end{array}$ & $\begin{array}{c}\text { SC } \\
(\mu \mathbf{S} / \mathbf{c m})\end{array}$ & $\begin{array}{c}\text { DOsat } \\
\text { (percent) }\end{array}$ & $\begin{array}{c}\text { DO } \\
\text { (mg/L) }\end{array}$ & $\begin{array}{c}\text { pH } \\
\text { (standard } \\
\text { units) }\end{array}$ \\
\hline $7 / 27 / 1999$ & $8: 30: 40$ & 24.01 & 441 & 105.6 & 8.9 & 8.2 \\
\hline $7 / 27 / 1999$ & 9:00:40 & 23.64 & 445 & 83.8 & 7.1 & 8.1 \\
\hline $7 / 27 / 1999$ & 9:30:40 & 23.69 & 445 & 84 & 7.1 & 8.0 \\
\hline $7 / 27 / 1999$ & 10:00:40 & 23.87 & 443 & 90.5 & 7.6 & 8.1 \\
\hline $7 / 27 / 1999$ & $10: 30: 40$ & 23.69 & 444 & 85.4 & 7.2 & 8.1 \\
\hline $7 / 27 / 1999$ & 11:00:40 & 23.92 & 443 & 93.4 & 7.9 & 8.1 \\
\hline $7 / 27 / 1999$ & 11:30:40 & 23.71 & 446 & 86.2 & 7.3 & 8.0 \\
\hline $7 / 27 / 1999$ & $12: 00: 40$ & 23.51 & 447 & 75.8 & 6.4 & 8.0 \\
\hline $7 / 27 / 1999$ & $12: 30: 40$ & 23.43 & 447 & 74 & 6.3 & 7.9 \\
\hline $7 / 27 / 1999$ & 13:00:40 & 24.03 & 446 & 88.1 & 7.4 & 8.0 \\
\hline $7 / 27 / 1999$ & $13: 30: 40$ & 23.95 & 446 & 82.6 & 7.0 & 8.0 \\
\hline $7 / 27 / 1999$ & 14:00:40 & 23.96 & 447 & 80.3 & 6.8 & 8.0 \\
\hline $7 / 27 / 1999$ & $14: 30: 40$ & 23.85 & 448 & 81.2 & 6.9 & 8.0 \\
\hline $7 / 27 / 1999$ & $15: 00: 40$ & 23.59 & 449 & 75.1 & 6.4 & 7.9 \\
\hline $7 / 27 / 1999$ & $15: 30: 40$ & 23.99 & 449 & 78.3 & 6.6 & 7.9 \\
\hline $7 / 27 / 1999$ & $16: 00: 40$ & 23.7 & 450 & 74.2 & 6.3 & 7.9 \\
\hline $7 / 27 / 1999$ & $16: 30: 40$ & 23.53 & 450 & 85.7 & 7.3 & 7.9 \\
\hline $7 / 27 / 1999$ & 17:00:40 & 23.93 & 449 & 68.1 & $5.7^{\mathbf{b}}$ & 7.9 \\
\hline $7 / 27 / 1999$ & $17: 30: 40$ & 23.55 & 448 & 69.1 & 5.9 & 7.9 \\
\hline $7 / 27 / 1999$ & 18:00:40 & 23.54 & 441 & 89.8 & 7.6 & 8.0 \\
\hline $7 / 27 / 1999$ & $18: 30: 40$ & 23.62 & 441 & 89.2 & 7.6 & 8.1 \\
\hline $7 / 27 / 1999$ & 19:00:40 & 23.7 & 440 & 106.1 & 9.0 & 8.2 \\
\hline $7 / 27 / 1999$ & $19: 30: 40$ & 24.02 & 441 & 102.7 & 8.6 & 8.2 \\
\hline $7 / 27 / 1999$ & 20:00:40 & 24.12 & 435 & 112.9 & 9.5 & 8.2 \\
\hline $7 / 27 / 1999$ & $20: 30: 40$ & 24.45 & 433 & 122.2 & 10.2 & 8.3 \\
\hline 7/27/1999 & 21:00:40 & 24.39 & 435 & 147.7 & 12.3 & 8.5 \\
\hline $7 / 27 / 1999$ & $21: 30: 40$ & 24.5 & 435 & 153.5 & 12.8 & 8.5 \\
\hline $7 / 27 / 1999$ & $22: 00: 40$ & 24.68 & 436 & 157.5 & $13.1^{\mathrm{a}}$ & 8.5 \\
\hline $7 / 27 / 1999$ & $22: 30: 40$ & 24.81 & 434 & 157.6 & 13.1 & 8.6 \\
\hline $7 / 27 / 1999$ & $23: 00: 40$ & 24.97 & 432 & 157.6 & 13.0 & 8.6 \\
\hline $7 / 27 / 1999$ & $23: 30: 40$ & 24.98 & 434 & 154.9 & 12.8 & 8.6 \\
\hline $7 / 28 / 1999$ & 0:00:40 & 25.02 & 433 & 152.3 & 12.6 & 8.6 \\
\hline $7 / 28 / 1999$ & $0: 30: 40$ & 25 & 433 & 150.4 & 12.4 & 8.5 \\
\hline $7 / 28 / 1999$ & 1:00:40 & 24.98 & 434 & 147.3 & 12.2 & 8.5 \\
\hline $7 / 28 / 1999$ & $1: 30: 40$ & 25.02 & 434 & 142.3 & 11.7 & 8.5 \\
\hline $7 / 28 / 1999$ & 2:00:40 & 25 & 435 & 137.5 & 11.4 & 8.5 \\
\hline $7 / 28 / 1999$ & $2: 30: 40$ & 24.94 & 435 & 134.8 & 11.1 & 8.5 \\
\hline $7 / 28 / 1999$ & 3:00:40 & 24.93 & 435 & 131.4 & 10.9 & 8.5 \\
\hline $7 / 28 / 1999$ & $3: 30: 40$ & 24.89 & 435 & 129.7 & 10.7 & 8.4 \\
\hline $7 / 28 / 1999$ & 4:00:40 & 24.84 & 435 & 128.4 & 10.6 & 8.4 \\
\hline $7 / 28 / 1999$ & 4:30:40 & 24.75 & 435 & 124.8 & 10.4 & 8.4 \\
\hline $7 / 28 / 1999$ & 5:00:40 & 24.61 & 436 & 122.1 & 10.2 & 8.4 \\
\hline $7 / 28 / 1999$ & $5: 30: 40$ & 24.56 & 437 & 111 & 9.2 & 8.3 \\
\hline $7 / 28 / 1999$ & 6:00:40 & 24.25 & 441 & 94.5 & 7.9 & 8.1 \\
\hline $7 / 28 / 1999$ & $6: 30: 40$ & 24.04 & 441 & 80.2 & 6.7 & 8.0 \\
\hline $7 / 28 / 1999$ & 7:00:40 & 23.98 & 442 & 79.3 & 6.7 & 8.0 \\
\hline $7 / 28 / 1999$ & $7: 30: 40$ & 23.79 & 442 & 74 & 6.3 & 8.0 \\
\hline $7 / 28 / 1999$ & 8:00:40 & 23.7 & 445 & 73 & 6.2 & 8.0 \\
\hline
\end{tabular}


Water-Quality Synoptic Sampling, July 1999: North Fork Shenandoah River, Virginia

Appendix Continued

\begin{tabular}{|c|c|c|c|c|c|c|}
\hline Date & Time & $\begin{array}{c}\text { Water } \\
\text { temperature } \\
\text { ('C) }\end{array}$ & $\begin{array}{c}\text { SC } \\
(\mu \mathbf{S} / \mathbf{c m})\end{array}$ & $\begin{array}{c}\text { DOsat } \\
\text { (percent) }\end{array}$ & $\begin{array}{c}\mathrm{DO} \\
(\mathrm{mg} / \mathrm{L})\end{array}$ & $\begin{array}{c}\mathrm{pH} \\
\text { (standard } \\
\text { units) }\end{array}$ \\
\hline $7 / 26 / 1999$ & $14: 30: 40$ & 23.43 & 445 & 102.2 & 8.7 & 8.1 \\
\hline $7 / 26 / 1999$ & $15: 00: 40$ & 23.46 & 445 & 101.8 & 8.6 & 8.1 \\
\hline $7 / 26 / 1999$ & $15: 30: 40$ & 23.41 & 445 & 102.3 & 8.7 & 8.1 \\
\hline $7 / 26 / 1999$ & $16: 00: 40$ & 23.41 & 444 & 102.8 & 8.7 & 8.1 \\
\hline $7 / 26 / 1999$ & $16: 30: 40$ & 23.42 & 444 & 101.1 & 8.6 & 8.1 \\
\hline $7 / 26 / 1999$ & $17: 00: 40$ & 23.32 & 444 & 100.2 & 8.5 & 8.1 \\
\hline $7 / 26 / 1999$ & $17: 30: 40$ & 23.33 & 443 & 100.7 & 8.6 & 8.1 \\
\hline $7 / 26 / 1999$ & 18:00:40 & 23.27 & 443 & 98.9 & 8.4 & 8.1 \\
\hline $7 / 26 / 1999$ & $18: 30: 40$ & 23.21 & 443 & 96 & 8.2 & 8.1 \\
\hline $7 / 26 / 1999$ & 19:00:40 & 23.19 & 443 & 94.9 & 8.1 & 8.1 \\
\hline $7 / 26 / 1999$ & $19: 30: 40$ & 23.2 & 443 & 92.4 & 7.9 & 8.1 \\
\hline $7 / 26 / 1999$ & 20:00:40 & 23.29 & 443 & 90.3 & 7.7 & 8.1 \\
\hline $7 / 26 / 1999$ & $20: 30: 40$ & 23.24 & 443 & 89.2 & 7.6 & 8.1 \\
\hline $7 / 26 / 1999$ & 21:00:40 & 23.27 & 443 & 88.1 & 7.5 & 8.1 \\
\hline $7 / 26 / 1999$ & $21: 30: 40$ & 23.24 & 443 & 88.7 & 7.6 & 8.1 \\
\hline $7 / 26 / 1999$ & $22: 00: 40$ & 23.26 & 442 & 88.8 & 7.6 & 8.1 \\
\hline $7 / 26 / 1999$ & $22: 30: 40$ & 23.28 & 442 & 88 & 7.5 & 8.1 \\
\hline $7 / 26 / 1999$ & $23: 00: 40$ & 23.36 & 442 & 87.7 & 7.5 & 8.1 \\
\hline $7 / 26 / 1999$ & $23: 30: 40$ & 23.36 & 442 & 88 & 7.5 & 8.1 \\
\hline $7 / 27 / 1999$ & 0:00:40 & 23.39 & 441 & 88.1 & 7.5 & 8.1 \\
\hline $7 / 27 / 1999$ & 0:30:40 & 23.39 & 442 & 88.4 & 7.5 & 8.1 \\
\hline $7 / 27 / 1999$ & 1:00:40 & 23.4 & 442 & 89 & 7.6 & 8.2 \\
\hline $7 / 27 / 1999$ & $1: 30: 40$ & 23.45 & 441 & 89.3 & 7.6 & 8.2 \\
\hline 7/27/1999 & 2:00:40 & 23.49 & 441 & 89.3 & 7.6 & 8.2 \\
\hline $7 / 27 / 1999$ & $2: 30: 40$ & 23.44 & 441 & 88.5 & 7.5 & 8.2 \\
\hline $7 / 27 / 1999$ & 3:00:40 & 23.39 & 441 & 88.3 & 7.5 & 8.1 \\
\hline $7 / 27 / 1999$ & $3: 30: 40$ & 23.44 & 441 & 88.2 & 7.5 & 8.2 \\
\hline $7 / 27 / 1999$ & 4:00:40 & 23.4 & 441 & 87.9 & 7.5 & 8.1 \\
\hline $7 / 27 / 1999$ & 4:30:40 & 23.41 & 441 & 87.9 & 7.5 & 8.1 \\
\hline $7 / 27 / 1999$ & 5:00:40 & 23.4 & 441 & 87 & 7.4 & 8.1 \\
\hline $7 / 27 / 1999$ & $5: 30: 40$ & 23.38 & 440 & 85.9 & 7.3 & 8.1 \\
\hline 7/27/1999 & 6:00:40 & 23.39 & 439 & 84.7 & 7.2 & 8.1 \\
\hline $7 / 27 / 1999$ & $6: 30: 40$ & 23.31 & 439 & 83.9 & 7.2 & 8.0 \\
\hline $7 / 27 / 1999$ & 7:00:40 & 23.28 & 439 & 83.8 & 7.1 & 8.0 \\
\hline 7/27/1999 & 7:30:40 & 23.23 & 438 & 83.3 & $7.1^{\mathbf{b}}$ & 8.0 \\
\hline $7 / 27 / 1999$ & 8:00:40 & 23.16 & 438 & 83.9 & 7.2 & 8.0 \\
\hline $7 / 27 / 1999$ & 8:30:40 & 23.16 & 438 & 87.4 & 7.5 & 8.0 \\
\hline $7 / 27 / 1999$ & 9:00:40 & 23.11 & 439 & 89 & 7.6 & 8.0 \\
\hline $7 / 27 / 1999$ & $9: 30: 40$ & 23.08 & 439 & 91.2 & 7.8 & 8.0 \\
\hline $7 / 27 / 1999$ & 10:00:40 & 23.08 & 439 & 95.2 & 8.1 & 8.0 \\
\hline $7 / 27 / 1999$ & $10: 30: 40$ & 23.07 & 439 & 95.6 & 8.2 & 8.0 \\
\hline $7 / 27 / 1999$ & 11:00:40 & 23.2 & 440 & 100.4 & 8.6 & 8.1 \\
\hline $7 / 27 / 1999$ & $11: 30: 40$ & 23.14 & 439 & 101 & 8.6 & 8.1 \\
\hline $7 / 27 / 1999$ & $12: 00: 40$ & 23.29 & 439 & 104.4 & 8.9 & 8.1 \\
\hline $7 / 27 / 1999$ & $12: 30: 40$ & 23.31 & 438 & 105.4 & 9.0 & 8.1 \\
\hline $7 / 27 / 1999$ & 13:00:40 & 23.29 & 439 & 105.2 & 9.0 & 8.1 \\
\hline $7 / 27 / 1999$ & $13: 30: 40$ & 23.21 & 438 & 104.5 & 8.9 & 8.1 \\
\hline $7 / 27 / 1999$ & $14: 00: 40$ & 22.98 & 439 & 99.7 & 8.6 & 8.1 \\
\hline $7 / 27 / 1999$ & $14: 30: 40$ & 23.09 & 439 & 103.6 & 8.9 & 8.1 \\
\hline $7 / 27 / 1999$ & $15: 00: 40$ & 23.17 & 438 & 105.4 & $9.0^{\mathbf{a}}$ & 8.1 \\
\hline
\end{tabular}


Appendix Continued

\begin{tabular}{|c|c|c|c|c|c|c|}
\hline Date & Time & $\begin{array}{c}\text { Water } \\
\text { temperature } \\
\text { ('C) }\end{array}$ & $\begin{array}{c}\text { SC } \\
(\mu \mathrm{S} / \mathrm{cm})\end{array}$ & $\begin{array}{c}\text { DOsat } \\
\text { (percent) }\end{array}$ & $\begin{array}{c}\text { DO } \\
\text { (mg/L) }\end{array}$ & $\begin{array}{c}\mathrm{pH} \\
\text { (standard } \\
\text { units) }\end{array}$ \\
\hline $7 / 27 / 1999$ & $15: 30: 40$ & 22.97 & 438 & 100.1 & 8.6 & 8.1 \\
\hline 7/27/1999 & 16:00:40 & 22.9 & 438 & 99.1 & 8.5 & 8.1 \\
\hline $7 / 27 / 1999$ & $16: 30: 40$ & 23.05 & 438 & 103.2 & 8.8 & 8.1 \\
\hline 7/27/1999 & 17:00:40 & 23.09 & 437 & 103.6 & 8.9 & 8.1 \\
\hline $7 / 27 / 1999$ & $17: 30: 40$ & 22.96 & 438 & 98.5 & 8.5 & 8.1 \\
\hline $7 / 27 / 1999$ & 18:00:40 & 22.94 & 438 & 98.1 & 8.4 & 8.1 \\
\hline $7 / 27 / 1999$ & $18: 30: 40$ & 22.97 & 437 & 99.4 & 8.5 & 8.1 \\
\hline $7 / 27 / 1999$ & 19:00:40 & 23.01 & 437 & 97.6 & 8.4 & 8.1 \\
\hline $7 / 27 / 1999$ & $19: 30: 40$ & 22.97 & 437 & 93.8 & 8.0 & 8.1 \\
\hline 7/27/1999 & 20:00:40 & 22.99 & 438 & 88.8 & 7.6 & 8.1 \\
\hline $7 / 27 / 1999$ & $20: 30: 40$ & 22.99 & 437 & 86 & 7.4 & 8.0 \\
\hline $7 / 27 / 1999$ & 21:00:40 & 22.96 & 438 & 84.8 & 7.3 & 8.0 \\
\hline $7 / 27 / 1999$ & $21: 30: 40$ & 23.07 & 437 & 86.7 & 7.4 & 8.1 \\
\hline $7 / 27 / 1999$ & $22: 00: 40$ & 23.07 & 438 & 86 & 7.4 & 8.1 \\
\hline $7 / 27 / 1999$ & $22: 30: 40$ & 23.09 & 437 & 86.5 & 7.4 & 8.1 \\
\hline 7/27/1999 & 23:00:40 & 23.18 & 437 & 87.4 & 7.5 & 8.1 \\
\hline 7/27/1999 & $23: 30: 40$ & 23.21 & 437 & 88.4 & 7.5 & 8.1 \\
\hline $7 / 28 / 1999$ & 0:00:40 & 23.27 & 437 & 88.8 & 7.6 & 8.1 \\
\hline 7/28/1999 & $0: 30: 40$ & 23.24 & 437 & 87.8 & 7.5 & 8.1 \\
\hline $7 / 28 / 1999$ & 1:00:40 & 23.25 & 437 & 88.3 & 7.5 & 8.1 \\
\hline $7 / 28 / 1999$ & 1:30:40 & 23.33 & 436 & 88.8 & 7.6 & 8.1 \\
\hline $7 / 28 / 1999$ & 2:00:40 & 23.29 & 437 & 88.4 & 7.5 & 8.1 \\
\hline $7 / 28 / 1999$ & 2:30:40 & 23.31 & 437 & 88.1 & 7.5 & 8.1 \\
\hline $7 / 28 / 1999$ & 3:00:40 & 23.39 & 437 & 88.1 & 7.5 & 8.1 \\
\hline $7 / 28 / 1999$ & $3: 30: 40$ & 23.33 & 437 & 87.8 & 7.5 & 8.1 \\
\hline $7 / 28 / 1999$ & 4:00:40 & 23.41 & 436 & 88.4 & 7.5 & 8.1 \\
\hline $7 / 28 / 1999$ & 4:30:40 & 23.42 & 436 & 88.6 & 7.5 & 8.1 \\
\hline $7 / 28 / 1999$ & 5:00:40 & 23.42 & 436 & 88.2 & 7.5 & 8.1 \\
\hline $7 / 28 / 1999$ & $5: 30: 40$ & 23.37 & 436 & 87.8 & 7.5 & 8.1 \\
\hline $7 / 28 / 1999$ & 6:00:40 & 23.38 & 436 & 87.3 & 7.4 & 8.1 \\
\hline $7 / 28 / 1999$ & $6: 30: 40$ & 23.37 & 436 & 86 & 7.3 & 8.0 \\
\hline $7 / 28 / 1999$ & 7:00:40 & 23.32 & 436 & 85.3 & 7.3 & 8.0 \\
\hline $7 / 28 / 1999$ & 7:30:40 & 23.3 & 436 & 87 & 7.4 & 8.0 \\
\hline $7 / 28 / 1999$ & 8:00:40 & 23.29 & 436 & 88.7 & 7.6 & 8.0 \\
\hline \multicolumn{7}{|c|}{ Site 32} \\
\hline $7 / 12 / 1999$ & 19:00:40 & 21.35 & 469 & 111.4 & 9.9 & 8.3 \\
\hline 7/12/1999 & $19: 30: 40$ & 21.28 & 470 & 110 & 9.7 & 8.3 \\
\hline $7 / 12 / 1999$ & 20:00:40 & 21.21 & 470 & 108.6 & 9.6 & 8.3 \\
\hline 7/12/1999 & $20: 30: 40$ & 21.14 & 470 & 107.5 & 9.6 & 8.3 \\
\hline 7/12/1999 & 21:00:40 & 21.06 & 471 & 105.4 & 9.4 & 8.3 \\
\hline 7/12/1999 & $21: 30: 40$ & 21 & 471 & 103.8 & 9.2 & 8.3 \\
\hline $7 / 12 / 1999$ & $22: 00: 40$ & 20.93 & 471 & 100.9 & 9.0 & 8.3 \\
\hline 7/12/1999 & $22: 30: 40$ & 20.86 & 472 & 98.4 & 8.8 & 8.3 \\
\hline $7 / 12 / 1999$ & 23:00:40 & 20.8 & 472 & 96.1 & 8.6 & 8.3 \\
\hline $7 / 12 / 1999$ & $23: 30: 40$ & 20.75 & 472 & 93 & 8.3 & 8.3 \\
\hline 7/13/1999 & 0:00:40 & 20.7 & 473 & 91 & 8.2 & 8.3 \\
\hline 7/13/1999 & 0:30:40 & 20.66 & 473 & 88.1 & 7.9 & 8.2 \\
\hline 7/13/1999 & 1:00:40 & 20.61 & 474 & 86.3 & 7.7 & 8.2 \\
\hline 7/13/1999 & 1:30:40 & 20.54 & 474 & 84.4 & 7.6 & 8.2 \\
\hline
\end{tabular}


Water-Quality Synoptic Sampling, July 1999: North Fork Shenandoah River, Virginia

Appendix Continued

\begin{tabular}{|c|c|c|c|c|c|c|}
\hline Date & Time & $\begin{array}{c}\text { Water } \\
\text { temperature } \\
\text { ('C) }\end{array}$ & $\begin{array}{c}\text { SC } \\
(\mu \mathrm{S} / \mathbf{c m})\end{array}$ & $\begin{array}{c}\text { D0sat } \\
\text { (percent) }\end{array}$ & $\begin{array}{c}\text { DO } \\
\text { (mg/L) }\end{array}$ & $\begin{array}{c}\text { pH } \\
\text { (standard } \\
\text { units) }\end{array}$ \\
\hline $7 / 13 / 1999$ & $2: 00: 40$ & 20.49 & 475 & 81.3 & 7.3 & 8.2 \\
\hline 7/13/1999 & $2: 30: 40$ & 20.45 & 475 & 79 & 7.1 & 8.2 \\
\hline 7/13/1999 & 3:00:40 & 20.38 & 477 & 76.7 & 6.9 & 8.1 \\
\hline 7/13/1999 & $3: 30: 40$ & 20.33 & 478 & 74.8 & 6.7 & 8.1 \\
\hline 7/13/1999 & 4:00:40 & 20.29 & 478 & 72.9 & 6.6 & 8.1 \\
\hline 7/13/1999 & 4:30:40 & 20.25 & 478 & 70.9 & 6.4 & 8.1 \\
\hline 7/13/1999 & 5:00:40 & 20.21 & 478 & 69.7 & 6.3 & 8.1 \\
\hline 7/13/1999 & $5: 30: 40$ & 20.18 & 478 & 68.2 & 6.2 & 8.0 \\
\hline 7/13/1999 & 6:00:40 & 20.11 & 478 & 67.6 & 6.1 & 8.0 \\
\hline 7/13/1999 & $6: 30: 40$ & 20.08 & 479 & 66.1 & 6.0 & 8.0 \\
\hline 7/13/1999 & 7:00:40 & 20.07 & 479 & 65.5 & 5.9 & 8.0 \\
\hline 7/13/1999 & 7:30:40 & 20.05 & 479 & 66.7 & 6.1 & 8.0 \\
\hline 7/13/1999 & 8:00:40 & 20.05 & 479 & 67.5 & 6.1 & 8.0 \\
\hline 7/13/1999 & 8:30:40 & 20.05 & 478 & 70.2 & 6.4 & 8.0 \\
\hline 7/13/1999 & 9:00:40 & 20.1 & 479 & 73.9 & 6.7 & 8.0 \\
\hline 7/13/1999 & $9: 30: 40$ & 20.14 & 478 & 78.2 & 7.1 & 8.0 \\
\hline 7/13/1999 & 10:00:40 & 20.3 & 478 & 82.8 & 7.5 & 8.1 \\
\hline 7/13/1999 & $10: 30: 40$ & 20.51 & 478 & 89 & 8.0 & 8.1 \\
\hline 7/13/1999 & 11:00:40 & 20.53 & 478 & 89.1 & 8.0 & 8.1 \\
\hline 7/13/1999 & 11:30:40 & 20.81 & 477 & 93.7 & 8.4 & 8.2 \\
\hline 7/13/1999 & $12: 00: 40$ & 21 & 476 & 99.3 & 8.8 & 8.2 \\
\hline 7/13/1999 & $12: 30: 40$ & 21.21 & 476 & 106.9 & 9.5 & 8.2 \\
\hline 7/13/1999 & 13:00:40 & 21.43 & 475 & 112.3 & 9.9 & 8.3 \\
\hline 7/13/1999 & $13: 30: 40$ & 21.77 & 474 & 119.7 & 10.5 & 8.3 \\
\hline 7/13/1999 & 14:00:40 & 21.99 & 473 & 125.8 & 11.0 & 8.4 \\
\hline 7/13/1999 & $14: 30: 40$ & 22.21 & 472 & 131.6 & 11.4 & 8.4 \\
\hline 7/13/1999 & 15:00:40 & 22.57 & 471 & 137.6 & 11.9 & 8.5 \\
\hline 7/13/1999 & $15: 30: 40$ & 22.76 & 470 & 142 & 12.2 & 8.5 \\
\hline 7/13/1999 & $16: 00: 40$ & 23 & 468 & 148.2 & 12.7 & 8.6 \\
\hline 7/13/1999 & $16: 30: 40$ & 23.04 & 467 & 152.1 & 13.0 & 8.6 \\
\hline 7/13/1999 & 17:00:40 & 23.24 & 466 & 152.9 & 13.0 & 8.6 \\
\hline 7/13/1999 & $17: 30: 40$ & 23.39 & 465 & 159.9 & 13.6 & 8.7 \\
\hline 7/13/1999 & 18:00:40 & 23.46 & 464 & 160.7 & 13.6 & 8.7 \\
\hline 7/13/1999 & $18: 30: 40$ & 23.53 & 464 & 160.5 & 13.6 & 8.7 \\
\hline 7/13/1999 & 19:00:40 & 23.64 & 462 & 161.5 & 13.7 & 8.7 \\
\hline 7/13/1999 & $19: 30: 40$ & 23.69 & 462 & 159.7 & 13.5 & 8.7 \\
\hline 7/13/1999 & 20:00:40 & 23.72 & 461 & 156.9 & 13.3 & 8.8 \\
\hline 7/13/1999 & $20: 30: 40$ & 23.73 & 460 & 154.4 & 13.1 & 8.8 \\
\hline 7/13/1999 & 21:00:40 & 23.73 & 460 & 150.8 & 12.7 & 8.8 \\
\hline 7/13/1999 & $21: 30: 40$ & 23.72 & 459 & 146.1 & 12.4 & 8.8 \\
\hline 7/13/1999 & 22:00:40 & 23.67 & 459 & 141.1 & 11.9 & 8.8 \\
\hline 7/13/1999 & $22: 30: 40$ & 23.62 & 459 & 137.6 & 11.7 & 8.8 \\
\hline 7/13/1999 & $23: 00: 40$ & 23.55 & 459 & 132.5 & 11.2 & 8.8 \\
\hline 7/13/1999 & $23: 30: 40$ & 23.47 & 459 & 126.5 & 10.7 & 8.8 \\
\hline 7/14/1999 & 0:00:40 & 23.38 & 459 & 121.7 & 10.4 & 8.8 \\
\hline 7/14/1999 & 0:30:40 & 23.27 & 460 & 115.4 & 9.8 & 8.7 \\
\hline $7 / 14 / 1999$ & 1:00:40 & 23.23 & 460 & 112.2 & 9.6 & 8.7 \\
\hline 7/14/1999 & $1: 30: 40$ & 23.12 & 461 & 105.3 & 9.0 & 8.7 \\
\hline 7/14/1999 & 2:00:40 & 23.04 & 462 & 100.1 & 8.6 & 8.7 \\
\hline 7/14/1999 & 2:30:40 & 22.94 & 463 & 95.1 & 8.2 & 8.7 \\
\hline
\end{tabular}


Appendix Continued

\begin{tabular}{|c|c|c|c|c|c|c|}
\hline Date & Time & $\begin{array}{c}\text { Water } \\
\text { temperature } \\
\text { ('C) }\end{array}$ & $\begin{array}{c}\text { SC } \\
(\mu \mathrm{S} / \mathbf{c m})\end{array}$ & $\begin{array}{c}\text { DOsat } \\
\text { (percent) }\end{array}$ & $\begin{array}{c}\text { DO } \\
\text { (mg/L) }\end{array}$ & $\begin{array}{c}\text { pH } \\
\text { (standard } \\
\text { units) }\end{array}$ \\
\hline $7 / 14 / 1999$ & 3:00:40 & 22.85 & 463 & 91.5 & 7.9 & 8.6 \\
\hline 7/14/1999 & $3: 30: 40$ & 22.75 & 456 & 85.9 & 7.4 & 8.6 \\
\hline 7/14/1999 & 4:00:40 & 22.66 & 462 & 82.5 & 7.1 & 8.5 \\
\hline $7 / 14 / 1999$ & 4:30:40 & 22.6 & 458 & 80.3 & 6.9 & 8.5 \\
\hline 7/14/1999 & 5:00:40 & 22.51 & 455 & 76.3 & 6.6 & 8.5 \\
\hline 7/14/1999 & 5:30:40 & 22.41 & 469 & 73.2 & 6.4 & 8.4 \\
\hline 7/14/1999 & 6:00:40 & 22.34 & 469 & 71.3 & 6.2 & 8.4 \\
\hline $7 / 14 / 1999$ & $6: 30: 40$ & 22.24 & 470 & 68.6 & 6.0 & 8.4 \\
\hline 7/14/1999 & 7:00:40 & 22.18 & 471 & 61.8 & 5.4 & 8.3 \\
\hline $7 / 14 / 1999$ & $7: 30: 40$ & 22.14 & 471 & 36.7 & $3.2^{\mathbf{b}}$ & 8.3 \\
\hline $7 / 14 / 1999$ & 8:00:40 & 22.08 & 467 & 63 & 5.5 & 8.3 \\
\hline 7/14/1999 & 8:30:40 & 22.05 & 467 & 64 & 5.6 & 8.3 \\
\hline 7/14/1999 & 9:00:40 & 22.03 & 464 & 61.2 & 5.3 & 8.2 \\
\hline 7/14/1999 & 9:30:40 & 21.99 & 461 & 63.8 & 5.6 & 8.2 \\
\hline $7 / 14 / 1999$ & 10:00:40 & 21.99 & 472 & 66.4 & 5.8 & 8.2 \\
\hline $7 / 14 / 1999$ & $10: 30: 40$ & 22 & 472 & 70.2 & 6.1 & 8.2 \\
\hline 7/14/1999 & 11:00:40 & 22 & 472 & 76.8 & 6.7 & 8.2 \\
\hline $7 / 14 / 1999$ & 11:30:40 & 22.03 & 472 & 77.6 & 6.8 & 8.3 \\
\hline $7 / 14 / 1999$ & $12: 00: 40$ & 22.05 & 472 & 67 & 5.9 & 8.3 \\
\hline 7/14/1999 & $12: 30: 40$ & 22.04 & 463 & 91.7 & 8.0 & 8.3 \\
\hline 7/14/1999 & 13:00:40 & 22.09 & 471 & 97.4 & 8.5 & 8.3 \\
\hline $7 / 14 / 1999$ & $13: 30: 40$ & 22.18 & 471 & 103 & 9.0 & 8.4 \\
\hline $7 / 14 / 1999$ & $14: 00: 40$ & 22.37 & 470 & 110.4 & 9.6 & 8.4 \\
\hline 7/14/1999 & $14: 30: 40$ & 22.49 & 470 & 105.9 & 9.2 & 8.4 \\
\hline 7/14/1999 & $15: 00: 40$ & 22.75 & 470 & 119.6 & 10.3 & 8.5 \\
\hline 7/14/1999 & $15: 30: 40$ & 22.82 & 469 & 126.4 & 10.9 & 8.5 \\
\hline 7/14/1999 & $16: 00: 40$ & 22.97 & 468 & 130.1 & 11.2 & 8.5 \\
\hline 7/14/1999 & $16: 30: 40$ & 23.05 & 467 & 134.8 & 11.5 & 8.6 \\
\hline 7/14/1999 & 17:00:40 & 23.17 & 466 & 140.5 & 12.0 & 8.6 \\
\hline $7 / 14 / 1999$ & $17: 30: 40$ & 23.27 & 465 & 147.2 & 12.6 & 8.6 \\
\hline $7 / 14 / 1999$ & 18:00:40 & 23.33 & 465 & 148.2 & 12.6 & 8.7 \\
\hline $7 / 14 / 1999$ & 18:30:40 & 23.36 & 464 & 151.4 & 12.9 & 8.7 \\
\hline 7/14/1999 & 19:00:40 & 23.34 & 463 & 150.9 & 12.8 & 8.7 \\
\hline 7/14/1999 & $19: 30: 40$ & 23.28 & 463 & 150 & 12.8 & 8.7 \\
\hline 7/14/1999 & 20:00:40 & 23.25 & 463 & 147.2 & 12.6 & 8.7 \\
\hline 7/14/1999 & $20: 30: 40$ & 23.23 & 462 & 145.2 & 12.4 & 8.7 \\
\hline $7 / 14 / 1999$ & 21:00:40 & 23.21 & 462 & 142.3 & 12.1 & 8.8 \\
\hline 7/14/1999 & 21:30:40 & 23.17 & 462 & 138.8 & 11.9 & 8.8 \\
\hline 7/14/1999 & 22:00:40 & 23.12 & 462 & 135 & 11.5 & 8.8 \\
\hline 7/14/1999 & $22: 30: 40$ & 23.03 & 462 & 130.6 & 11.2 & 8.8 \\
\hline 7/14/1999 & 23:00:40 & 22.96 & 462 & 126.2 & 10.8 & 8.7 \\
\hline 7/14/1999 & $23: 30: 40$ & 22.89 & 463 & 121.5 & 10.4 & 8.7 \\
\hline $7 / 15 / 1999$ & 0:00:40 & 22.79 & 464 & 116.4 & 10.0 & 8.7 \\
\hline $7 / 15 / 1999$ & 0:30:40 & 22.72 & 464 & 113.3 & 9.8 & 8.7 \\
\hline $7 / 15 / 1999$ & 1:00:40 & 22.65 & 464 & 104.4 & 9.0 & 8.7 \\
\hline 7/15/1999 & $1: 30: 40$ & 22.56 & 465 & 102.5 & 8.9 & 8.7 \\
\hline 7/15/1999 & 2:00:40 & 22.47 & 466 & 98.9 & 8.6 & 8.7 \\
\hline 7/15/1999 & 2:30:40 & 22.39 & 467 & 95.7 & 8.3 & 8.6 \\
\hline 7/15/1999 & 3:00:40 & 22.31 & 468 & 91.7 & 8.0 & 8.6 \\
\hline $7 / 15 / 1999$ & $3: 30: 40$ & 22.23 & 469 & 86.7 & 7.5 & 8.6 \\
\hline
\end{tabular}


Water-Quality Synoptic Sampling, July 1999: North Fork Shenandoah River, Virginia

Appendix Continued

\begin{tabular}{|c|c|c|c|c|c|c|}
\hline Date & Time & $\begin{array}{c}\text { Water } \\
\text { temperature } \\
\left.\text { ( }{ }^{\circ} \mathrm{C}\right)\end{array}$ & $\begin{array}{c}\text { SC } \\
(\mu \mathbf{S} / \mathbf{c m})\end{array}$ & $\begin{array}{c}\text { DOsat } \\
\text { (percent) }\end{array}$ & $\begin{array}{c}\text { DO } \\
\text { (mg/L) }\end{array}$ & $\begin{array}{c}\mathrm{pH} \\
\text { (standard } \\
\text { units) }\end{array}$ \\
\hline $7 / 15 / 1999$ & 4:00:40 & 22.12 & 470 & 83.4 & 7.3 & 8.5 \\
\hline 7/15/1999 & $4: 30: 40$ & 22.06 & 470 & 81.4 & 7.1 & 8.5 \\
\hline 7/15/1999 & 5:00:40 & 21.97 & 471 & 77.9 & 6.8 & 8.5 \\
\hline 7/15/1999 & $5: 30: 40$ & 21.86 & 472 & 74.8 & 6.6 & 8.4 \\
\hline $7 / 15 / 1999$ & 6:00:40 & 21.77 & 473 & 72.2 & 6.3 & 8.4 \\
\hline $7 / 15 / 1999$ & 6:30:40 & 21.65 & 473 & 69.7 & 6.1 & 8.4 \\
\hline $7 / 15 / 1999$ & 7:00:40 & 21.59 & 474 & 62.3 & 5.5 & 8.4 \\
\hline $7 / 15 / 1999$ & 7:30:40 & 21.51 & 474 & 68 & 6.0 & 8.3 \\
\hline $7 / 15 / 1999$ & 8:00:40 & 21.51 & 474 & 67.5 & 6.0 & 8.4 \\
\hline 7/15/1999 & $8: 30: 40$ & 21.49 & 473 & 69.8 & 6.2 & 8.4 \\
\hline $7 / 15 / 1999$ & 9:00:40 & 21.53 & 473 & 69.6 & 6.1 & 8.4 \\
\hline 7/15/1999 & 9:30:40 & 21.59 & 473 & 75 & 6.6 & 8.4 \\
\hline 7/15/1999 & 10:00:40 & 21.76 & 472 & 82.3 & 7.2 & 8.4 \\
\hline $7 / 15 / 1999$ & $10: 30: 40$ & 21.92 & 472 & 87.8 & 7.7 & 8.4 \\
\hline 7/15/1999 & 11:00:40 & 22.04 & 471 & 89.3 & 7.8 & 8.4 \\
\hline 7/15/1999 & $11: 30: 40$ & 22.21 & 471 & 94.6 & 8.2 & 8.4 \\
\hline 7/15/1999 & $12: 00: 40$ & 22.41 & 470 & 99.8 & 8.6 & 8.5 \\
\hline 7/15/1999 & $12: 30: 40$ & 22.61 & 470 & 106.9 & 9.2 & 8.5 \\
\hline $7 / 15 / 1999$ & $13: 00: 40$ & 22.83 & 469 & 110.5 & 9.5 & 8.5 \\
\hline $7 / 15 / 1999$ & $13: 30: 40$ & 23.06 & 469 & 119.3 & 10.2 & 8.6 \\
\hline $7 / 15 / 1999$ & 14:00:40 & 23.37 & 263 & 130.6 & 11.1 & 8.6 \\
\hline 7/15/1999 & $14: 30: 40$ & 23.53 & 262 & 131.8 & 11.2 & 8.6 \\
\hline $7 / 15 / 1999$ & $15: 00: 40$ & 23.92 & 262 & 144.7 & 12.2 & 8.7 \\
\hline $7 / 15 / 1999$ & $15: 30: 40$ & 24.17 & 262 & 152 & 12.7 & 8.7 \\
\hline $7 / 15 / 1999$ & 16:00:40 & 24.35 & 261 & 155.3 & 13.0 & 8.7 \\
\hline $7 / 15 / 1999$ & $16: 30: 40$ & 24.57 & 261 & 158.8 & 13.2 & 8.7 \\
\hline $7 / 15 / 1999$ & 17:00:40 & 24.71 & 260 & 162.9 & 13.5 & 8.8 \\
\hline $7 / 15 / 1999$ & $17: 30: 40$ & 24.83 & 260 & 162.4 & 13.5 & 8.8 \\
\hline 7/15/1999 & 18:00:40 & 25 & 260 & 166.5 & 13.8 & 8.8 \\
\hline $7 / 15 / 1999$ & $18: 30: 40$ & 25.06 & 260 & 163 & 13.4 & 8.8 \\
\hline 7/15/1999 & 19:00:40 & 25.15 & 259 & 163.9 & 13.5 & 8.8 \\
\hline $7 / 15 / 1999$ & $19: 30: 40$ & 25.2 & 259 & 159.1 & 13.1 & 8.8 \\
\hline $7 / 15 / 1999$ & 20:00:40 & 25.24 & 259 & 154.9 & 12.7 & 8.8 \\
\hline $7 / 15 / 1999$ & $20: 30: 40$ & 25.3 & 259 & 149.6 & 12.3 & 8.9 \\
\hline 7/15/1999 & 21:00:40 & 25.31 & 258 & 148.3 & 12.2 & 8.9 \\
\hline $7 / 15 / 1999$ & $21: 30: 40$ & 25.29 & 257 & 144.2 & 11.8 & 8.9 \\
\hline $7 / 15 / 1999$ & $22: 00: 40$ & 25.25 & 257 & 139.1 & 11.4 & 8.9 \\
\hline $7 / 15 / 1999$ & $22: 30: 40$ & 25.2 & 256 & 133 & 10.9 & 8.9 \\
\hline $7 / 15 / 1999$ & 23:00:40 & 25.12 & 257 & 129.4 & 10.7 & 8.8 \\
\hline $7 / 15 / 1999$ & $23: 30: 40$ & 25.05 & 257 & 124.3 & 10.3 & 8.8 \\
\hline $7 / 16 / 1999$ & 0:00:40 & 24.99 & 257 & 118.6 & 9.8 & 8.8 \\
\hline 7/16/1999 & $0: 30: 40$ & 24.92 & 257 & 111.5 & 9.2 & 8.8 \\
\hline 7/16/1999 & 1:00:40 & 24.84 & 257 & 107.4 & 8.9 & 8.8 \\
\hline $7 / 16 / 1999$ & $1: 30: 40$ & 24.77 & 258 & 99.3 & 8.2 & 8.8 \\
\hline 7/16/1999 & 2:00:40 & 24.69 & 258 & 98 & 8.1 & 8.7 \\
\hline 7/16/1999 & 2:30:40 & 24.62 & 258 & 92.5 & 7.7 & 8.7 \\
\hline 7/16/1999 & 3:00:40 & 24.52 & 259 & 85.8 & 7.2 & 8.7 \\
\hline 7/16/1999 & $3: 30: 40$ & 24.41 & 381 & 83.2 & 7.0 & 8.7 \\
\hline 7/16/1999 & 4:00:40 & 24.31 & 416 & 77.2 & 6.5 & 8.6 \\
\hline 7/16/1999 & 4:30:40 & 24.18 & 426 & 73.2 & 6.1 & 8.6 \\
\hline
\end{tabular}


Appendix Continued

\begin{tabular}{|c|c|c|c|c|c|c|}
\hline Date & Time & $\begin{array}{c}\text { Water } \\
\text { temperature } \\
\left.\text { ( }{ }^{\circ} \mathrm{C}\right)\end{array}$ & $\begin{array}{c}\text { SC } \\
(\mu \mathbf{S} / \mathbf{c m})\end{array}$ & $\begin{array}{c}\text { D0sat } \\
\text { (percent) }\end{array}$ & $\begin{array}{c}\mathrm{DO} \\
(\mathrm{mg} / \mathrm{L})\end{array}$ & $\begin{array}{c}\text { pH } \\
\text { (standard } \\
\text { units) }\end{array}$ \\
\hline $7 / 16 / 1999$ & $5: 00: 40$ & 24.08 & 431 & 70.6 & 5.9 & 8.5 \\
\hline 7/16/1999 & $5: 30: 40$ & 23.98 & 428 & 67.4 & 5.7 & 8.5 \\
\hline 7/16/1999 & 6:00:40 & 23.87 & 461 & 63.3 & 5.3 & 8.5 \\
\hline 7/16/1999 & $6: 30: 40$ & 23.79 & 460 & 62 & 5.2 & 8.4 \\
\hline $7 / 16 / 1999$ & 7:00:40 & 23.7 & 461 & 62 & 5.2 & 8.4 \\
\hline $7 / 16 / 1999$ & $7: 30: 40$ & 23.61 & 458 & 64.6 & 5.5 & 8.4 \\
\hline 7/16/1999 & 8:00:40 & 23.56 & 459 & 65.2 & 5.5 & 8.4 \\
\hline 7/16/1999 & 8:30:40 & 23.6 & 466 & 70.6 & 6.0 & 8.4 \\
\hline $7 / 16 / 1999$ & 9:00:40 & 23.63 & 466 & 76.2 & 6.5 & 8.4 \\
\hline 7/16/1999 & 9:30:40 & 23.73 & 466 & 81.2 & 6.9 & 8.5 \\
\hline $7 / 16 / 1999$ & 10:00:40 & 23.84 & 466 & 87 & 7.3 & 8.5 \\
\hline $7 / 16 / 1999$ & $10: 30: 40$ & 23.97 & 465 & 93.5 & 7.9 & 8.5 \\
\hline 7/16/1999 & 11:00:40 & 24.05 & 465 & 99.8 & 8.4 & 8.5 \\
\hline $7 / 16 / 1999$ & 11:30:40 & 24.22 & 465 & 105.8 & 8.9 & 8.6 \\
\hline $7 / 16 / 1999$ & 12:00:40 & 24.39 & 464 & 111.9 & 9.3 & 8.6 \\
\hline $7 / 16 / 1999$ & $12: 30: 40$ & 24.54 & 463 & 115.5 & 9.6 & 8.6 \\
\hline 7/16/1999 & 13:00:40 & 24.74 & 463 & 121.8 & 10.1 & 8.6 \\
\hline 7/16/1999 & $13: 30: 40$ & 24.93 & 462 & 128 & 10.6 & 8.6 \\
\hline $7 / 16 / 1999$ & 14:00:40 & 25.16 & 462 & 136 & 11.2 & 8.7 \\
\hline $7 / 16 / 1999$ & $14: 30: 40$ & 25.42 & 460 & 144.7 & 11.9 & 8.7 \\
\hline $7 / 16 / 1999$ & $15: 00: 40$ & 25.55 & 460 & 149.3 & 12.2 & 8.7 \\
\hline 7/16/1999 & $15: 30: 40$ & 25.74 & 459 & 153.4 & 12.5 & 8.7 \\
\hline 7/16/1999 & $16: 00: 40$ & 26.03 & 457 & 162 & 13.1 & 8.8 \\
\hline 7/16/1999 & $16: 30: 40$ & 26.19 & 456 & 165.6 & 13.4 & 8.8 \\
\hline $7 / 16 / 1999$ & 17:00:40 & 26.35 & 455 & 170.2 & 13.7 & 8.8 \\
\hline $7 / 16 / 1999$ & $17: 30: 40$ & 26.48 & 454 & 172.5 & 13.9 & 8.8 \\
\hline 7/16/1999 & 18:00:40 & 26.58 & 453 & 174 & 14.0 & 8.9 \\
\hline 7/16/1999 & $18: 30: 40$ & 26.65 & 452 & 174.4 & 14.0 & 8.9 \\
\hline $7 / 16 / 1999$ & 19:00:40 & 26.67 & 451 & 171.2 & 13.7 & 8.9 \\
\hline 7/16/1999 & 19:30:40 & 26.63 & 451 & 165.5 & 13.3 & 8.9 \\
\hline 7/16/1999 & 20:00:40 & 26.57 & 451 & 157.1 & 12.6 & 8.9 \\
\hline 7/16/1999 & $20: 30: 40$ & 26.51 & 450 & 151.9 & 12.2 & 8.9 \\
\hline $7 / 16 / 1999$ & 21:00:40 & 26.45 & 450 & 148.7 & 12.0 & 8.9 \\
\hline $7 / 16 / 1999$ & $21: 30: 40$ & 26.37 & 450 & 143.7 & 11.6 & 8.9 \\
\hline $7 / 16 / 1999$ & $22: 00: 40$ & 26.29 & 450 & 137.8 & 11.1 & 8.9 \\
\hline 7/16/1999 & $22: 30: 40$ & 26.19 & 451 & 132.3 & 10.7 & 8.9 \\
\hline $7 / 16 / 1999$ & $23: 00: 40$ & 26.09 & 451 & 128.3 & 10.4 & 8.9 \\
\hline 7/16/1999 & $23: 30: 40$ & 26 & 451 & 121.9 & 9.9 & 8.9 \\
\hline 7/17/1999 & 0:00:40 & 25.91 & 452 & 116.2 & 9.4 & 8.8 \\
\hline 7/17/1999 & 0:30:40 & 25.82 & 453 & 109.2 & 8.9 & 8.8 \\
\hline 7/17/1999 & 1:00:40 & 25.74 & 453 & 103.4 & 8.4 & 8.8 \\
\hline 7/17/1999 & $1: 30: 40$ & 25.64 & 455 & 96.3 & 7.9 & 8.8 \\
\hline 7/17/1999 & 2:00:40 & 25.55 & 456 & 90.2 & 7.4 & 8.7 \\
\hline $7 / 17 / 1999$ & $2: 30: 40$ & 25.46 & 456 & 88.5 & 7.3 & 8.7 \\
\hline 7/17/1999 & 3:00:40 & 25.33 & 458 & 80.7 & 6.6 & 8.7 \\
\hline 7/17/1999 & $3: 30: 40$ & 25.24 & 459 & 77.4 & 6.4 & 8.6 \\
\hline 7/17/1999 & 4:00:40 & 25.12 & 460 & 72.3 & 6.0 & 8.6 \\
\hline 7/17/1999 & 4:30:40 & 25 & 461 & 68.9 & 5.7 & 8.6 \\
\hline $7 / 17 / 1999$ & 5:00:40 & 24.88 & 462 & 65.8 & 5.4 & 8.5 \\
\hline 7/17/1999 & $5: 30: 40$ & 24.77 & 463 & 62.4 & 5.2 & 8.5 \\
\hline
\end{tabular}


Water-Quality Synoptic Sampling, July 1999: North Fork Shenandoah River, Virginia

Appendix Continued

\begin{tabular}{|c|c|c|c|c|c|c|}
\hline Date & Time & $\begin{array}{c}\text { Water } \\
\text { temperature } \\
\text { ('C) }\end{array}$ & $\begin{array}{c}\text { SC } \\
(\mu \mathbf{S} / \mathbf{c m})\end{array}$ & $\begin{array}{c}\text { D0sat } \\
\text { (percent) }\end{array}$ & $\begin{array}{c}\text { DO } \\
\text { (mg/L) }\end{array}$ & $\begin{array}{c}\text { pH } \\
\text { (standard } \\
\text { units) }\end{array}$ \\
\hline $7 / 17 / 1999$ & $6: 00: 40$ & 24.67 & 464 & 59.4 & 4.9 & 8.5 \\
\hline 7/17/1999 & $6: 30: 40$ & 24.58 & 464 & 57.2 & 4.8 & 8.4 \\
\hline $7 / 17 / 1999$ & 7:00:40 & 24.49 & 465 & 60.5 & 5.0 & 8.4 \\
\hline 7/17/1999 & $7: 30: 40$ & 24.42 & 454 & 64.1 & 5.4 & 8.4 \\
\hline 7/17/1999 & 8:00:40 & 24.38 & 464 & 67.4 & 5.6 & 8.4 \\
\hline 7/17/1999 & $8: 30: 40$ & 24.39 & 467 & 74.6 & 6.2 & 8.5 \\
\hline 7/17/1999 & 9:00:40 & 24.45 & 465 & 80.2 & 6.7 & 8.5 \\
\hline 7/17/1999 & $9: 30: 40$ & 24.52 & 464 & 82.4 & 6.9 & 8.5 \\
\hline 7/17/1999 & 10:00:40 & 24.66 & 464 & 93.7 & 7.8 & 8.6 \\
\hline 7/17/1999 & $10: 30: 40$ & 24.8 & 464 & 96 & 8.0 & 8.6 \\
\hline 7/17/1999 & 11:00:40 & 24.89 & 463 & 99.5 & 8.2 & 8.6 \\
\hline 7/17/1999 & $11: 30: 40$ & 25.02 & 463 & 110.1 & 9.1 & 8.7 \\
\hline 7/17/1999 & $12: 00: 40$ & 25.12 & 463 & 111.2 & 9.2 & 8.7 \\
\hline $7 / 17 / 1999$ & $12: 30: 40$ & 25.35 & 462 & 119.4 & 9.8 & 8.8 \\
\hline $7 / 17 / 1999$ & 13:00:40 & 25.6 & 461 & 126.7 & 10.4 & 8.8 \\
\hline $7 / 17 / 1999$ & $13: 30: 40$ & 25.75 & 461 & 135.1 & 11.0 & 8.7 \\
\hline 7/17/1999 & 14:00:40 & 26.02 & 459 & 140 & 11.3 & 8.8 \\
\hline 7/17/1999 & $14: 30: 40$ & 26.28 & 457 & 147.8 & 11.9 & 8.8 \\
\hline 7/17/1999 & 15:00:40 & 26.5 & 457 & 148.9 & 12.0 & 8.7 \\
\hline 7/17/1999 & $15: 30: 40$ & 26.72 & 456 & 158.1 & 12.7 & 8.8 \\
\hline $7 / 17 / 1999$ & $16: 00: 40$ & 26.96 & 455 & 163.6 & 13.0 & 8.8 \\
\hline $7 / 17 / 1999$ & $16: 30: 40$ & 27.13 & 453 & 169.7 & 13.5 & 8.8 \\
\hline 7/17/1999 & 17:00:40 & 27.28 & 452 & 173.5 & 13.7 & 8.9 \\
\hline $7 / 17 / 1999$ & $17: 30: 40$ & 27.33 & 451 & 171.2 & 13.6 & 8.8 \\
\hline 7/17/1999 & 18:00:40 & 27.36 & 451 & 167.7 & 13.3 & 8.8 \\
\hline 7/17/1999 & $18: 30: 40$ & 27.35 & 451 & 163.4 & 12.9 & 8.9 \\
\hline 7/17/1999 & 19:00:40 & 27.38 & 450 & 160.6 & 12.7 & 8.9 \\
\hline 7/17/1999 & $19: 30: 40$ & 27.35 & 450 & 154.4 & 12.2 & 8.9 \\
\hline $7 / 17 / 1999$ & 20:00:40 & 27.28 & 449 & 147.9 & 11.7 & 8.9 \\
\hline $7 / 17 / 1999$ & $20: 30: 40$ & 27.2 & 449 & 140.5 & 11.2 & 8.8 \\
\hline $7 / 17 / 1999$ & 21:00:40 & 27.1 & 449 & 134.9 & 10.7 & 8.8 \\
\hline 7/17/1999 & $21: 30: 40$ & 27.02 & 448 & 127.1 & 10.1 & 8.8 \\
\hline 7/17/1999 & $22: 00: 40$ & 26.91 & 450 & 129.5 & 10.3 & 8.8 \\
\hline 7/17/1999 & $22: 30: 40$ & 26.79 & 449 & 125 & 10.0 & 8.8 \\
\hline 7/17/1999 & 23:00:40 & 26.69 & 450 & 119 & 9.5 & 8.8 \\
\hline $7 / 17 / 1999$ & $23: 30: 40$ & 26.6 & 451 & 111.9 & 9.0 & 8.8 \\
\hline $7 / 18 / 1999$ & 0:00:40 & 26.5 & 452 & 104.4 & 8.4 & 8.7 \\
\hline $7 / 18 / 1999$ & $0: 30: 40$ & 26.42 & 452 & 99.3 & 8.0 & 8.7 \\
\hline $7 / 18 / 1999$ & 1:00:40 & 26.35 & 454 & 92.6 & 7.5 & 8.7 \\
\hline $7 / 18 / 1999$ & 1:30:40 & 26.29 & 454 & 89.7 & 7.2 & 8.7 \\
\hline $7 / 18 / 1999$ & 2:00:40 & 26.19 & 456 & 84.5 & 6.8 & 8.6 \\
\hline $7 / 18 / 1999$ & $2: 30: 40$ & 26.1 & 457 & 78.8 & 6.4 & 8.6 \\
\hline $7 / 18 / 1999$ & 3:00:40 & 26 & 457 & 74.3 & 6.0 & 8.5 \\
\hline $7 / 18 / 1999$ & $3: 30: 40$ & 25.91 & 458 & 71.3 & 5.8 & 8.5 \\
\hline $7 / 18 / 1999$ & 4:00:40 & 25.8 & 459 & 66.1 & 5.4 & 8.5 \\
\hline $7 / 18 / 1999$ & 4:30:40 & 25.68 & 460 & 64.7 & 5.3 & 8.5 \\
\hline $7 / 18 / 1999$ & 5:00:40 & 25.57 & 461 & 60.5 & 4.9 & 8.3 \\
\hline $7 / 18 / 1999$ & $5: 30: 40$ & 25.47 & 462 & 58.4 & 4.8 & 8.4 \\
\hline $7 / 18 / 1999$ & 6:00:40 & 25.39 & 462 & 55.6 & 4.6 & 8.4 \\
\hline 7/18/1999 & $6: 30: 40$ & 25.27 & 463 & 53.6 & 4.4 & 8.3 \\
\hline
\end{tabular}


Appendix Continued

\begin{tabular}{|c|c|c|c|c|c|c|}
\hline Date & Time & $\begin{array}{c}\text { Water } \\
\text { temperature } \\
\text { (ㄷ) }\end{array}$ & $\begin{array}{c}\text { SC } \\
(\mu \mathbf{S} / \mathbf{c m})\end{array}$ & $\begin{array}{c}\text { DOsat } \\
\text { (percent) }\end{array}$ & $\begin{array}{c}\text { DO } \\
\text { (mg/L) }\end{array}$ & $\begin{array}{c}\text { pH } \\
\text { (standard } \\
\text { units) }\end{array}$ \\
\hline $7 / 18 / 1999$ & $7: 00: 40$ & 25.19 & 463 & 52.4 & 4.3 & 8.3 \\
\hline 7/18/1999 & 7:30:40 & 25.14 & 463 & 54.9 & 4.5 & 8.4 \\
\hline $7 / 18 / 1999$ & 8:00:40 & 25.06 & 463 & 58.1 & 4.8 & 8.4 \\
\hline 7/18/1999 & $8: 30: 40$ & 25.04 & 463 & 59.9 & 4.9 & 8.4 \\
\hline 7/18/1999 & 9:00:40 & 25.05 & 463 & 72.2 & 6.0 & 8.4 \\
\hline 7/18/1999 & $9: 30: 40$ & 25.11 & 463 & 75 & 6.2 & 8.5 \\
\hline $7 / 18 / 1999$ & 10:00:40 & 25.2 & 463 & 82.7 & 6.8 & 8.5 \\
\hline $7 / 18 / 1999$ & $10: 30: 40$ & 25.29 & 461 & 88.3 & 7.3 & 8.6 \\
\hline 7/18/1999 & 11:00:40 & 25.46 & 460 & 97.4 & 8.0 & 8.7 \\
\hline 7/18/1999 & $11: 30: 40$ & 25.57 & 460 & 100.8 & 8.2 & 8.7 \\
\hline 7/18/1999 & $12: 00: 40$ & 25.69 & 460 & 105.2 & 8.6 & 8.5 \\
\hline 7/18/1999 & $12: 30: 40$ & 25.79 & 461 & 115.6 & 9.4 & 8.7 \\
\hline 7/18/1999 & 13:00:40 & 25.99 & 460 & 124.9 & 10.1 & 8.7 \\
\hline 7/18/1999 & $13: 30: 40$ & 26.24 & 458 & 133.9 & 10.8 & 8.8 \\
\hline $7 / 18 / 1999$ & 14:00:40 & 26.25 & 457 & 135.3 & 10.9 & 8.7 \\
\hline 7/18/1999 & $14: 30: 40$ & 26.49 & 457 & 144.3 & 11.6 & 8.8 \\
\hline $7 / 18 / 1999$ & 15:00:40 & 26.55 & 457 & 139.2 & 11.2 & 8.7 \\
\hline 7/18/1999 & $15: 30: 40$ & 26.65 & 456 & 144.5 & 11.6 & 8.7 \\
\hline $7 / 18 / 1999$ & $16: 00: 40$ & 26.75 & 455 & 152.4 & 12.2 & 8.8 \\
\hline 7/18/1999 & $16: 30: 40$ & 26.85 & 453 & 160.6 & 12.8 & 8.8 \\
\hline 7/18/1999 & $17: 00: 40$ & 26.95 & 452 & 167.9 & 13.4 & 8.9 \\
\hline $7 / 18 / 1999$ & $17: 30: 40$ & 27.02 & 451 & 169.9 & 13.5 & 8.9 \\
\hline 7/18/1999 & 18:00:40 & 27.04 & 451 & 165.6 & 13.2 & 8.9 \\
\hline 7/18/1999 & $18: 30: 40$ & 27.05 & 450 & 166 & 13.2 & 8.9 \\
\hline 7/18/1999 & 19:00:40 & 27.04 & 449 & 164.8 & 13.1 & 8.9 \\
\hline 7/18/1999 & $19: 30: 40$ & 27.01 & 449 & 155.3 & 12.4 & 8.8 \\
\hline 7/18/1999 & 20:00:40 & 26.97 & 449 & 151.7 & 12.1 & 8.9 \\
\hline 7/18/1999 & $20: 30: 40$ & 26.92 & 449 & 144.5 & 11.5 & 8.9 \\
\hline $7 / 18 / 1999$ & 21:00:40 & 26.87 & 449 & 141.7 & 11.3 & 8.9 \\
\hline 7/18/1999 & $21: 30: 40$ & 26.81 & 449 & 135.5 & 10.8 & 8.8 \\
\hline 7/18/1999 & 22:00:40 & 26.75 & 449 & 130 & 10.4 & 8.8 \\
\hline $7 / 18 / 1999$ & $22: 30: 40$ & 26.66 & 449 & 122.5 & 9.8 & 8.8 \\
\hline $7 / 18 / 1999$ & 23:00:40 & 26.58 & 449 & 119.5 & 9.6 & 8.8 \\
\hline 7/18/1999 & $23: 30: 40$ & 26.49 & 450 & 115.9 & 9.3 & 8.8 \\
\hline 7/19/1999 & 0:00:40 & 26.39 & 451 & 109.9 & 8.8 & 8.8 \\
\hline $7 / 19 / 1999$ & $0: 30: 40$ & 26.31 & 452 & 104 & 8.4 & 8.8 \\
\hline 7/19/1999 & 1:00:40 & 26.21 & 453 & 98 & 7.9 & 8.7 \\
\hline 7/19/1999 & $1: 30: 40$ & 26.11 & 453 & 94.7 & 7.7 & 8.7 \\
\hline 7/19/1999 & 2:00:40 & 26.01 & 454 & 89.7 & 7.3 & 8.7 \\
\hline $7 / 19 / 1999$ & $2: 30: 40$ & 25.91 & 455 & 83.1 & 6.7 & 8.7 \\
\hline 7/19/1999 & 3:00:40 & 25.8 & 457 & 77.5 & 6.3 & 8.6 \\
\hline $7 / 19 / 1999$ & $3: 30: 40$ & 25.69 & 458 & 70.7 & 5.8 & 8.6 \\
\hline 7/19/1999 & 4:00:40 & 25.62 & 459 & 69.4 & 5.7 & 8.6 \\
\hline 7/19/1999 & 4:30:40 & 25.47 & 459 & 66.3 & 5.4 & 8.5 \\
\hline 7/19/1999 & 5:00:40 & 25.35 & 461 & 62.5 & 5.1 & 8.5 \\
\hline 7/19/1999 & $5: 30: 40$ & 25.27 & 462 & 58.1 & 4.8 & 8.4 \\
\hline $7 / 19 / 1999$ & 6:00:40 & 25.14 & 463 & 55.4 & 4.6 & 8.4 \\
\hline 7/19/1999 & $6: 30: 40$ & 25.05 & 464 & 54.7 & 4.5 & 8.3 \\
\hline 7/19/1999 & 7:00:40 & 24.97 & 464 & 55.7 & 4.6 & 8.3 \\
\hline 7/19/1999 & 7:30:40 & 24.93 & 464 & 61.6 & 5.1 & 8.4 \\
\hline
\end{tabular}


Water-Quality Synoptic Sampling, July 1999: North Fork Shenandoah River, Virginia

Appendix Continued

\begin{tabular}{|c|c|c|c|c|c|c|}
\hline Date & Time & $\begin{array}{c}\text { Water } \\
\text { temperature } \\
\text { ('C) }\end{array}$ & $\begin{array}{c}\text { SC } \\
(\mu \mathbf{S} / \mathbf{c m})\end{array}$ & $\begin{array}{c}\text { DOsat } \\
\text { (percent) }\end{array}$ & $\begin{array}{c}\mathrm{DO} \\
\text { (mg/L) }\end{array}$ & $\begin{array}{c}\mathrm{pH} \\
\text { (standard } \\
\text { units) }\end{array}$ \\
\hline $7 / 19 / 1999$ & $8: 00: 40$ & 24.88 & 464 & 71 & 5.9 & 8.4 \\
\hline 7/19/1999 & 8:30:40 & 24.87 & 464 & 82.9 & 6.9 & 8.5 \\
\hline $7 / 19 / 1999$ & 9:00:40 & 24.92 & 464 & 89.7 & 7.4 & 8.6 \\
\hline 7/19/1999 & 9:30:40 & 24.99 & 463 & 97.4 & 8.0 & 8.6 \\
\hline $7 / 19 / 1999$ & 10:00:40 & 25.1 & 463 & 107 & 8.8 & 8.7 \\
\hline 7/19/1999 & $10: 30: 40$ & 25.23 & 462 & 121.6 & 10.0 & 8.7 \\
\hline $7 / 19 / 1999$ & 11:00:40 & 25.36 & 461 & 122.2 & 10.0 & 8.7 \\
\hline $7 / 19 / 1999$ & 11:30:40 & 25.51 & 461 & 126.1 & 10.3 & 8.8 \\
\hline $7 / 19 / 1999$ & $12: 00: 40$ & 25.67 & 460 & 131.3 & 10.7 & 8.9 \\
\hline 7/19/1999 & $12: 30: 40$ & 25.7 & 460 & 120.8 & 9.8 & 8.7 \\
\hline 7/19/1999 & $13: 00: 40$ & 25.83 & 460 & 128.8 & 10.5 & 8.9 \\
\hline $7 / 19 / 1999$ & $13: 30: 40$ & 26.04 & 459 & 126.8 & 10.3 & 8.7 \\
\hline $7 / 19 / 1999$ & $14: 00: 40$ & 26.16 & 457 & 146.5 & 11.8 & 8.8 \\
\hline $7 / 19 / 1999$ & $14: 30: 40$ & 26.29 & 457 & 151.8 & 12.2 & 8.8 \\
\hline $7 / 19 / 1999$ & $15: 00: 40$ & 26.52 & 456 & 154.3 & 12.4 & 8.8 \\
\hline $7 / 19 / 1999$ & $15: 30: 40$ & 26.66 & 455 & 162.1 & 13.0 & 8.8 \\
\hline $7 / 19 / 1999$ & $16: 00: 40$ & 26.76 & 455 & 158.7 & 12.7 & 8.7 \\
\hline $7 / 19 / 1999$ & $16: 30: 40$ & 26.95 & 453 & 180 & 14.3 & 8.9 \\
\hline $7 / 19 / 1999$ & $17: 00: 40$ & 27.04 & 452 & 177.3 & 14.1 & 8.8 \\
\hline 7/19/1999 & $17: 30: 40$ & 27.11 & 451 & 181.6 & $14.4^{\mathbf{a}}$ & 8.8 \\
\hline $7 / 19 / 1999$ & 18:00:40 & 27.14 & 451 & 174.2 & 13.8 & 8.9 \\
\hline $7 / 19 / 1999$ & $18: 30: 40$ & 27.18 & 451 & 162.6 & 12.9 & 8.9 \\
\hline $7 / 19 / 1999$ & 19:00:40 & 27.2 & 450 & 159.9 & 12.7 & 8.9 \\
\hline 7/19/1999 & $19: 30: 40$ & 27.19 & 450 & 154.2 & 12.2 & 8.8 \\
\hline 7/19/1999 & 20:00:40 & 27.15 & 450 & 148.2 & 11.8 & 8.8 \\
\hline 7/19/1999 & $20: 30: 40$ & 27.12 & 449 & 145.1 & 11.5 & 8.8 \\
\hline $7 / 19 / 1999$ & 21:00:40 & 27.07 & 450 & 140.5 & 11.2 & 8.8 \\
\hline 7/19/1999 & $21: 30: 40$ & 27.01 & 450 & 133.7 & 10.6 & 8.8 \\
\hline $7 / 19 / 1999$ & $22: 00: 40$ & 26.95 & 450 & 131.4 & 10.5 & 8.8 \\
\hline $7 / 19 / 1999$ & $22: 30: 40$ & 26.89 & 451 & 122.1 & 9.7 & 8.8 \\
\hline $7 / 19 / 1999$ & $23: 00: 40$ & 26.81 & 451 & 116.3 & 9.3 & 8.8 \\
\hline $7 / 19 / 1999$ & $23: 30: 40$ & 26.73 & 452 & 112.5 & 9.0 & 8.8 \\
\hline $7 / 20 / 1999$ & 0:00:40 & 26.64 & 452 & 108.8 & 8.7 & 8.7 \\
\hline $7 / 20 / 1999$ & $0: 30: 40$ & 26.57 & 455 & 99.4 & 8.0 & 8.7 \\
\hline $7 / 20 / 1999$ & 1:00:40 & 26.49 & 455 & 93.7 & 7.5 & 8.7 \\
\hline $7 / 20 / 1999$ & $1: 30: 40$ & 26.4 & 456 & 90.1 & 7.3 & 8.7 \\
\hline $7 / 20 / 1999$ & 2:00:40 & 26.32 & 457 & 81.6 & 6.6 & 8.7 \\
\hline $7 / 20 / 1999$ & $2: 30: 40$ & 26.22 & 457 & 74.4 & 6.0 & 8.6 \\
\hline $7 / 20 / 1999$ & 3:00:40 & 26.12 & 459 & 69.7 & 5.6 & 8.6 \\
\hline $7 / 20 / 1999$ & $3: 30: 40$ & 26.03 & 461 & 67.3 & 5.5 & 8.5 \\
\hline $7 / 20 / 1999$ & 4:00:40 & 25.94 & 461 & 63 & 5.1 & 8.5 \\
\hline $7 / 20 / 1999$ & 4:30:40 & 25.83 & 461 & 58 & 4.7 & 8.5 \\
\hline $7 / 20 / 1999$ & 5:00:40 & 25.71 & 461 & 54 & 4.4 & 8.4 \\
\hline $7 / 20 / 1999$ & $5: 30: 40$ & 25.63 & 462 & 50.6 & 4.1 & 8.4 \\
\hline $7 / 20 / 1999$ & 6:00:40 & 25.52 & 463 & 47.8 & 3.9 & 8.3 \\
\hline $7 / 20 / 1999$ & $6: 30: 40$ & 25.42 & 464 & 45.3 & 3.7 & 8.3 \\
\hline $7 / 20 / 1999$ & 7:00:40 & 25.34 & 464 & 44.2 & 3.6 & 8.3 \\
\hline $7 / 20 / 1999$ & 7:30:40 & 25.29 & 465 & 44.5 & 3.7 & 8.3 \\
\hline $7 / 20 / 1999$ & 8:00:40 & 25.22 & 465 & 43.6 & 3.6 & 8.3 \\
\hline $7 / 20 / 1999$ & $8: 30: 40$ & 25.16 & 465 & 48.7 & 4.0 & 8.3 \\
\hline
\end{tabular}


Appendix Continued

\begin{tabular}{ccccccc}
\hline Date & Time & $\begin{array}{c}\text { Water } \\
\text { temperature } \\
\text { ( } \mathbf{C} \text { ) }\end{array}$ & $\begin{array}{c}\text { SC } \\
(\mu \mathbf{S} / \mathbf{c m})\end{array}$ & $\begin{array}{c}\text { DOsat } \\
\text { (percent) }\end{array}$ & $\begin{array}{c}\text { DO } \\
\text { (mg/L) }\end{array}$ & $\begin{array}{c}\text { pH } \\
\text { (standard } \\
\text { units) }\end{array}$ \\
\hline $7 / 20 / 1999$ & $9: 00: 40$ & 25.15 & 465 & 54.9 & 4.5 & 8.3 \\
$7 / 20 / 1999$ & $9: 30: 40$ & 25.13 & 465 & 59.1 & 4.9 & 8.4 \\
$7 / 20 / 1999$ & $10: 00: 40$ & 25.21 & 465 & 77.5 & 6.4 & 8.6 \\
$7 / 20 / 1999$ & $10: 30: 40$ & 25.26 & 464 & 79 & 6.5 & 8.5 \\
$7 / 20 / 1999$ & $11: 00: 40$ & 25.39 & 463 & 83.8 & 6.9 & 8.6 \\
$7 / 20 / 1999$ & $11: 30: 40$ & 25.43 & 463 & 92.6 & 7.6 & 8.6 \\
$7 / 20 / 1999$ & $12: 00: 40$ & 25.56 & 463 & 92.8 & 7.6 & 8.6 \\
$7 / 20 / 1999$ & $12: 30: 40$ & 25.58 & 463 & 83.9 & 6.9 & 8.5 \\
$7 / 20 / 1999$ & $13: 00: 40$ & 25.71 & 463 & 111.6 & 9.1 & 8.7 \\
$7 / 20 / 1999$ & $13: 30: 40$ & 25.79 & 462 & 127.1 & 10.3 & 8.7 \\
$7 / 20 / 1999$ & $14: 00: 40$ & 25.88 & 460 & 125.8 & 10.2 & 8.7 \\
$7 / 20 / 1999$ & $14: 30: 40$ & 25.97 & 460 & 125.2 & 10.2 & 8.7 \\
$7 / 20 / 1999$ & $15: 00: 40$ & 26.1 & 459 & 142 & 11.5 & 8.8 \\
$7 / 20 / 1999$ & $15: 30: 40$ & 26.14 & 458 & 127.9 & 10.3 & 8.7 \\
$7 / 20 / 1999$ & $16: 00: 40$ & 26.24 & 457 & 139 & 11.2 & 8.7 \\
\hline
\end{tabular}

$\begin{array}{lllcccl}7 / 12 / 1999 & 18: 30: 40 & 21.4 & - & 104.4 & 9.2 & 8.3 \\ 7 / 12 / 1999 & 19: 00: 40 & 21.36 & - & 104.4 & 9.2 & 8.3 \\ 7 / 12 / 1999 & 19: 30: 40 & 21.24 & - & 103.3 & 9.2 & 8.3 \\ 7 / 12 / 1999 & 20: 00: 40 & 21.1 & - & 101.7 & 9.1 & 8.3 \\ 7 / 12 / 1999 & 20: 30: 40 & 21.01 & - & 100.4 & 9.0 & 8.3 \\ 7 / 12 / 1999 & 21: 00: 40 & 20.93 & - & 99.2 & 8.9 & 8.3 \\ 7 / 12 / 1999 & 21: 30: 40 & 20.86 & - & 97.5 & 8.7 & 8.3 \\ 7 / 12 / 1999 & 22: 00: 40 & 20.8 & - & 95.8 & 8.6 & 8.3 \\ 7 / 12 / 1999 & 22: 30: 40 & 20.77 & - & 95.1 & 8.5 & 8.3 \\ 7 / 12 / 1999 & 23: 00: 40 & 20.73 & - & 93.1 & 8.3 & 8.3 \\ 7 / 12 / 1999 & 23: 30: 40 & 20.68 & - & 92.1 & 8.3 & 8.3 \\ 7 / 13 / 1999 & 0: 00: 40 & 20.58 & - & 90.2 & 8.1 & 8.3 \\ 7 / 13 / 1999 & 0: 30: 40 & 20.5 & - & 88.3 & 8.0 & 8.2 \\ 7 / 13 / 1999 & 1: 00: 40 & 20.43 & - & 85.8 & 7.7 & 8.2 \\ 7 / 13 / 1999 & 1: 30: 40 & 20.38 & - & 84.5 & 7.6 & 8.2 \\ 7 / 13 / 1999 & 2: 00: 40 & 20.32 & - & 82.1 & 7.4 & 8.2 \\ 7 / 13 / 1999 & 2: 30: 40 & 20.31 & - & 80.9 & 7.3 & 8.2 \\ 7 / 13 / 1999 & 3: 00: 40 & 20.28 & - & 78.9 & 7.1 & 8.2 \\ 7 / 13 / 1999 & 3: 30: 40 & 20.22 & - & 76.9 & 7.0 & 8.1 \\ 7 / 13 / 1999 & 4: 00: 40 & 20.14 & - & 75.7 & 6.9 & 8.1 \\ 7 / 13 / 1999 & 4: 30: 40 & 20.07 & - & 73.9 & 6.7 & 8.1 \\ 7 / 13 / 1999 & 5: 00: 40 & 20.02 & - & 72.5 & 6.6 & 8.1 \\ 7 / 13 / 1999 & 5: 30: 40 & 19.97 & - & 71.2 & 6.5 & 8.1 \\ 7 / 13 / 1999 & 6: 00: 40 & 19.95 & - & 70.2 & 6.4 & 8.0 \\ 7 / 13 / 1999 & 6: 30: 40 & 19.94 & - & 69.5 & 6.3 & 8.0 \\ 7 / 13 / 1999 & 7: 00: 40 & 19.96 & - & 70.3 & 6.4 & 8.0 \\ 7 / 13 / 1999 & 7: 30: 40 & 19.95 & - & 71.7 & 6.5 & 8.0 \\ 7 / 13 / 1999 & 8: 00: 40 & 19.85 & - & 73.8 & 6.7 & 8.0 \\ 7 / 13 / 1999 & 8: 30: 40 & 19.84 & - & 75.2 & 6.9 & 8.0 \\ 7 / 13 / 1999 & 9: 00: 40 & 19.88 & - & 79.3 & 7.2 & 8.1 \\ 7 / 13 / 1999 & 9: 30: 40 & 19.97 & - & 83.2 & 7.6 & 8.1 \\ 7 / 13 / 1999 & 10: 00: 40 & 20.17 & - & 87.5 & 7.9 & 8.1\end{array}$


Water-Quality Synoptic Sampling, July 1999: North Fork Shenandoah River, Virginia

Appendix Continued

\begin{tabular}{|c|c|c|c|c|c|c|}
\hline Date & Time & $\begin{array}{c}\text { Water } \\
\text { temperature } \\
\text { ('C) }\end{array}$ & $\begin{array}{c}\text { SC } \\
(\mu \mathbf{S} / \mathbf{c m})\end{array}$ & $\begin{array}{c}\text { DOsat } \\
\text { (percent) }\end{array}$ & $\begin{array}{c}\mathrm{DO} \\
\text { (mg/L) }\end{array}$ & $\begin{array}{c}\mathrm{pH} \\
\text { (standard } \\
\text { units) }\end{array}$ \\
\hline $7 / 13 / 1999$ & $10: 30: 40$ & 20.49 & - & 93 & 8.4 & 8.1 \\
\hline 7/13/1999 & 11:00:40 & 20.67 & - & 97.3 & 8.7 & 8.2 \\
\hline $7 / 13 / 1999$ & 11:30:40 & 21.06 & - & 103.2 & 9.2 & 8.2 \\
\hline 7/13/1999 & $12: 00: 40$ & 21.37 & - & 108.6 & 9.6 & 8.3 \\
\hline $7 / 13 / 1999$ & $12: 30: 40$ & 21.58 & 480 & 115.6 & 10.2 & 8.3 \\
\hline 7/13/1999 & $13: 00: 40$ & 21.71 & 480 & 119 & 10.5 & 8.3 \\
\hline 7/13/1999 & $13: 30: 40$ & 22.17 & 479 & 126.3 & 11.0 & 8.4 \\
\hline $7 / 13 / 1999$ & 14:00:40 & 22.37 & 477 & 130.6 & 11.3 & 8.4 \\
\hline $7 / 13 / 1999$ & $14: 30: 40$ & 22.72 & 476 & 136 & 11.7 & 8.5 \\
\hline 7/13/1999 & 15:00:40 & 22.95 & 474 & 141.5 & 12.1 & 8.5 \\
\hline 7/13/1999 & $15: 30: 40$ & 23.02 & 473 & 144.8 & 12.4 & 8.5 \\
\hline $7 / 13 / 1999$ & $16: 00: 40$ & 23.26 & 472 & 148.7 & 12.7 & 8.6 \\
\hline 7/13/1999 & $16: 30: 40$ & 23.32 & 469 & 150.8 & 12.8 & 8.6 \\
\hline $7 / 13 / 1999$ & $17: 00: 40$ & 23.46 & 467 & 153.6 & 13.1 & 8.6 \\
\hline 7/13/1999 & $17: 30: 40$ & 23.6 & 465 & 154.7 & 13.1 & 8.7 \\
\hline 7/13/1999 & 18:00:40 & 23.67 & 462 & 155.2 & 13.1 & 8.7 \\
\hline 7/13/1999 & $18: 30: 40$ & 23.63 & 460 & 153.3 & 13.0 & 8.7 \\
\hline $7 / 13 / 1999$ & 19:00:40 & 23.59 & 459 & 151.8 & 12.9 & 8.7 \\
\hline $7 / 13 / 1999$ & $19: 30: 40$ & 23.52 & 458 & 148.9 & 12.6 & 8.7 \\
\hline 7/13/1999 & 20:00:40 & 23.47 & 457 & 145.4 & 12.4 & 8.7 \\
\hline 7/13/1999 & $20: 30: 40$ & 23.41 & 455 & 141.2 & 12.0 & 8.7 \\
\hline 7/13/1999 & 21:00:40 & 23.42 & 455 & 138.8 & 11.8 & 8.7 \\
\hline $7 / 13 / 1999$ & $21: 30: 40$ & 23.44 & 454 & 136.8 & 11.6 & 8.7 \\
\hline 7/13/1999 & $22: 00: 40$ & 23.44 & 453 & 133.2 & 11.3 & 8.7 \\
\hline 7/13/1999 & $22: 30: 40$ & 23.42 & 453 & 130.3 & 11.1 & 8.7 \\
\hline 7/13/1999 & $23: 00: 40$ & 23.34 & 453 & 126.6 & 10.8 & 8.7 \\
\hline $7 / 13 / 1999$ & $23: 30: 40$ & 23.24 & 454 & 122 & 10.4 & 8.7 \\
\hline 7/14/1999 & 0:00:40 & 23.15 & 455 & 118.7 & 10.1 & 8.7 \\
\hline $7 / 14 / 1999$ & 0:30:40 & 23.1 & 456 & 115.3 & 9.9 & 8.7 \\
\hline $7 / 14 / 1999$ & 1:00:40 & 23.05 & 457 & 111.5 & 9.5 & 8.7 \\
\hline $7 / 14 / 1999$ & $1: 30: 40$ & 22.99 & 458 & 107.5 & 9.2 & 8.6 \\
\hline 7/14/1999 & 2:00:40 & 22.92 & 460 & 104.1 & 8.9 & 8.6 \\
\hline $7 / 14 / 1999$ & 2:30:40 & 22.79 & 462 & 99.7 & 8.6 & 8.6 \\
\hline $7 / 14 / 1999$ & 3:00:40 & 22.68 & 464 & 95 & 8.2 & 8.6 \\
\hline 7/14/1999 & 3:30:40 & 22.59 & 465 & 91.6 & 7.9 & 8.6 \\
\hline $7 / 14 / 1999$ & 4:00:40 & 22.53 & 466 & 88.1 & 7.6 & 8.5 \\
\hline $7 / 14 / 1999$ & 4:30:40 & 22.48 & 467 & 84.6 & 7.3 & 8.5 \\
\hline $7 / 14 / 1999$ & 5:00:40 & 22.43 & 469 & 82 & 7.1 & 8.5 \\
\hline $7 / 14 / 1999$ & $5: 30: 40$ & 22.35 & 471 & 78.6 & 6.8 & 8.4 \\
\hline $7 / 14 / 1999$ & 6:00:40 & 22.26 & 472 & 75.7 & 6.6 & 8.4 \\
\hline $7 / 14 / 1999$ & $6: 30: 40$ & 22.15 & 475 & 73.6 & 6.4 & 8.4 \\
\hline 7/14/1999 & 7:00:40 & 22.06 & 476 & 72.3 & 6.3 & 8.3 \\
\hline $7 / 14 / 1999$ & $7: 30: 40$ & 22.02 & 477 & 71.4 & 6.2 & 8.3 \\
\hline $7 / 14 / 1999$ & 8:00:40 & 22.02 & 476 & 70.8 & 6.2 & 8.3 \\
\hline $7 / 14 / 1999$ & 8:30:40 & 22 & 477 & 71.8 & 6.3 & 8.3 \\
\hline $7 / 14 / 1999$ & 9:00:40 & 21.98 & 477 & 72.9 & 6.4 & 8.3 \\
\hline $7 / 14 / 1999$ & 9:30:40 & 21.94 & 477 & 75.3 & 6.6 & 8.3 \\
\hline $7 / 14 / 1999$ & 10:00:40 & 21.92 & 477 & 78.3 & 6.8 & 8.3 \\
\hline 7/14/1999 & $10: 30: 40$ & 21.94 & 477 & 82.2 & 7.2 & 8.3 \\
\hline 7/14/1999 & 11:00:40 & 21.96 & 477 & 84.8 & 7.4 & 8.3 \\
\hline
\end{tabular}


Appendix Continued

\begin{tabular}{|c|c|c|c|c|c|c|}
\hline Date & Time & $\begin{array}{c}\text { Water } \\
\text { temperature } \\
\left.\text { ( }{ }^{\circ} \mathrm{C}\right)\end{array}$ & $\begin{array}{c}\text { SC } \\
(\mu \mathrm{S} / \mathbf{c m})\end{array}$ & $\begin{array}{c}\text { DOsat } \\
\text { (percent) }\end{array}$ & $\begin{array}{c}\text { DO } \\
\text { (mg/L) }\end{array}$ & $\begin{array}{c}\text { pH } \\
\text { (standard } \\
\text { units) }\end{array}$ \\
\hline $7 / 14 / 1999$ & $11: 30: 40$ & 22.03 & 477 & 89 & 7.8 & 8.3 \\
\hline 7/14/1999 & $12: 00: 40$ & 22.05 & 476 & 91.5 & 8.0 & 8.3 \\
\hline 7/14/1999 & $12: 30: 40$ & 22.06 & 476 & 95 & 8.3 & 8.3 \\
\hline 7/14/1999 & 13:00:40 & 22.11 & 477 & 100 & 8.7 & 8.4 \\
\hline $7 / 14 / 1999$ & $13: 30: 40$ & 22.35 & 476 & 107.9 & 9.4 & 8.4 \\
\hline 7/14/1999 & 14:00:40 & 22.61 & 476 & 113.9 & 9.8 & 8.4 \\
\hline 7/14/1999 & $14: 30: 40$ & 22.7 & 475 & 120 & 10.3 & 8.5 \\
\hline 7/14/1999 & $15: 00: 40$ & 22.99 & 473 & 126.2 & 10.8 & 8.5 \\
\hline $7 / 14 / 1999$ & $15: 30: 40$ & 23.2 & 473 & 131 & 11.2 & 8.5 \\
\hline $7 / 14 / 1999$ & $16: 00: 40$ & 23.35 & 473 & 134.6 & 11.5 & 8.5 \\
\hline 7/14/1999 & $16: 30: 40$ & 23.45 & 472 & 139.6 & 11.9 & 8.6 \\
\hline $7 / 14 / 1999$ & 17:00:40 & 23.48 & 469 & 142.8 & 12.1 & 8.6 \\
\hline 7/14/1999 & $17: 30: 40$ & 23.51 & 470 & 142.7 & 12.1 & 8.6 \\
\hline 7/14/1999 & 18:00:40 & 23.52 & 298 & 150.1 & 12.7 & 8.6 \\
\hline $7 / 14 / 1999$ & $18: 30: 40$ & 23.5 & 291 & 149 & 12.7 & 8.6 \\
\hline $7 / 14 / 1999$ & 19:00:40 & 23.45 & 284 & 148.6 & 12.6 & 8.7 \\
\hline 7/14/1999 & $19: 30: 40$ & 23.32 & 279 & 145.7 & 12.4 & 8.7 \\
\hline 7/14/1999 & 20:00:40 & 23.19 & 274 & 141.4 & 12.1 & 8.7 \\
\hline $7 / 14 / 1999$ & $20: 30: 40$ & 23.06 & 271 & 136.8 & 11.7 & 8.6 \\
\hline 7/14/1999 & 21:00:40 & 22.97 & 269 & 133.3 & 11.4 & 8.6 \\
\hline 7/14/1999 & 21:30:40 & 22.92 & 267 & 130.8 & 11.2 & 8.7 \\
\hline 7/14/1999 & 22:00:40 & 22.87 & 266 & 128.3 & 11.0 & 8.7 \\
\hline 7/14/1999 & $22: 30: 40$ & 22.84 & 266 & 126.4 & 10.9 & 8.7 \\
\hline $7 / 14 / 1999$ & 23:00:40 & 22.79 & 267 & 123.5 & 10.6 & 8.7 \\
\hline 7/14/1999 & $23: 30: 40$ & 22.7 & 269 & 120.1 & 10.4 & 8.7 \\
\hline 7/15/1999 & 0:00:40 & 22.57 & 272 & 115.8 & 10.0 & 8.6 \\
\hline 7/15/1999 & 0:30:40 & 22.46 & 276 & 112.1 & 9.7 & 8.6 \\
\hline 7/15/1999 & $1: 00: 40$ & 22.38 & 280 & 108.5 & 9.4 & 8.6 \\
\hline $7 / 15 / 1999$ & $1: 30: 40$ & 22.32 & 285 & 105.4 & 9.2 & 8.6 \\
\hline $7 / 15 / 1999$ & 2:00:40 & 22.27 & 290 & 101.8 & 8.9 & 8.6 \\
\hline 7/15/1999 & $2: 30: 40$ & 22.21 & 294 & 97.9 & 8.5 & 8.6 \\
\hline $7 / 15 / 1999$ & 3:00:40 & 22.15 & 299 & 95.4 & 8.3 & 8.6 \\
\hline 7/15/1999 & 3:30:40 & 22.01 & 304 & 91.4 & 8.0 & 8.5 \\
\hline $7 / 15 / 1999$ & 4:00:40 & 21.91 & 308 & 88.7 & 7.8 & 8.5 \\
\hline 7/15/1999 & 4:30:40 & 21.83 & 314 & 85.8 & 7.5 & 8.5 \\
\hline 7/15/1999 & 5:00:40 & 21.75 & 318 & 82.7 & 7.3 & 8.5 \\
\hline $7 / 15 / 1999$ & $5: 30: 40$ & 21.69 & 323 & 80.2 & 7.1 & 8.4 \\
\hline 7/15/1999 & 6:00:40 & 21.63 & 328 & 77.4 & 6.8 & 8.4 \\
\hline 7/15/1999 & 6:30:40 & 21.57 & 333 & 76.1 & 6.7 & 8.4 \\
\hline 7/15/1999 & 7:00:40 & 21.47 & 338 & 75.7 & 6.7 & 8.4 \\
\hline $7 / 15 / 1999$ & 7:30:40 & 21.37 & 341 & 76.5 & 6.8 & 8.3 \\
\hline $7 / 15 / 1999$ & 8:00:40 & 21.34 & 344 & 79 & 7.0 & 8.3 \\
\hline $7 / 15 / 1999$ & $8: 30: 40$ & 21.36 & 347 & 81 & 7.2 & 8.3 \\
\hline $7 / 15 / 1999$ & 9:00:40 & 21.4 & 349 & 83 & 7.3 & 8.3 \\
\hline 7/15/1999 & 9:30:40 & 21.43 & 351 & 86.4 & 7.6 & 8.3 \\
\hline 7/15/1999 & 10:00:40 & 21.63 & 353 & 98 & 8.6 & 8.4 \\
\hline 7/15/1999 & $10: 30: 40$ & 21.78 & 354 & 105.9 & 9.3 & 8.4 \\
\hline 7/15/1999 & 11:00:40 & 22.08 & 353 & 112.8 & 9.8 & 8.4 \\
\hline 7/15/1999 & 11:30:40 & 22.37 & 353 & 118.9 & 10.3 & 8.5 \\
\hline 7/15/1999 & $12: 00: 40$ & 22.7 & 351 & 123.3 & 10.6 & 8.5 \\
\hline $7 / 15 / 1999$ & $12: 30: 40$ & 23 & 350 & 127.4 & 10.9 & 8.5 \\
\hline
\end{tabular}


Water-Quality Synoptic Sampling, July 1999: North Fork Shenandoah River, Virginia

Appendix Continued

\begin{tabular}{|c|c|c|c|c|c|c|}
\hline Date & Time & $\begin{array}{c}\text { Water } \\
\text { temperature } \\
\left.\text { ( }{ }^{\circ} \mathrm{C}\right)\end{array}$ & $\begin{array}{c}\text { SC } \\
(\mu \mathbf{S} / \mathbf{c m})\end{array}$ & $\begin{array}{c}\text { DOsat } \\
\text { (percent) }\end{array}$ & $\begin{array}{c}\text { DO } \\
\text { (mg/L) }\end{array}$ & $\begin{array}{c}\mathrm{pH} \\
\text { (standard } \\
\text { units) }\end{array}$ \\
\hline $7 / 15 / 1999$ & $13: 00: 40$ & 23.31 & 348 & 130.4 & 11.1 & 8.6 \\
\hline 7/15/1999 & $13: 30: 40$ & 23.62 & 344 & 135.1 & 11.4 & 8.6 \\
\hline 7/15/1999 & 14:00:40 & 23.92 & 468 & 139.3 & 11.7 & 8.6 \\
\hline 7/15/1999 & $14: 30: 40$ & 23.94 & 467 & 136.1 & 11.5 & 8.6 \\
\hline $7 / 15 / 1999$ & 15:00:40 & 24.23 & 466 & 145.7 & 12.2 & 8.6 \\
\hline $7 / 15 / 1999$ & $15: 30: 40$ & 24.42 & 465 & 150.5 & 12.6 & 8.6 \\
\hline $7 / 15 / 1999$ & 16:00:40 & 24.67 & 462 & 154.9 & 12.9 & 8.7 \\
\hline $7 / 15 / 1999$ & $16: 30: 40$ & 24.9 & 460 & 158.7 & 13.1 & 8.7 \\
\hline $7 / 15 / 1999$ & 17:00:40 & 25.01 & 457 & 161.9 & 13.4 & 8.7 \\
\hline 7/15/1999 & $17: 30: 40$ & 25.01 & 454 & 160.3 & 13.2 & 8.7 \\
\hline $7 / 15 / 1999$ & 18:00:40 & 25.1 & 451 & 161.8 & 13.3 & 8.8 \\
\hline 7/15/1999 & $18: 30: 40$ & 25.13 & 449 & 160.7 & 13.2 & 8.8 \\
\hline $7 / 15 / 1999$ & 19:00:40 & 25.09 & 447 & 158.3 & 13.1 & 8.8 \\
\hline $7 / 15 / 1999$ & $19: 30: 40$ & 25.06 & 444 & 155.4 & 12.8 & 8.8 \\
\hline $7 / 15 / 1999$ & 20:00:40 & 25.03 & 442 & 151.4 & 12.5 & 8.8 \\
\hline 7/15/1999 & $20: 30: 40$ & 25.01 & 442 & 146.5 & 12.1 & 8.8 \\
\hline 7/15/1999 & 21:00:40 & 25.03 & 460 & 143 & 11.8 & 8.8 \\
\hline 7/15/1999 & $21: 30: 40$ & 25.03 & 460 & 139.3 & 11.5 & 8.8 \\
\hline $7 / 15 / 1999$ & 22:00:40 & 25 & 460 & 135.6 & 11.2 & 8.8 \\
\hline $7 / 15 / 1999$ & $22: 30: 40$ & 24.96 & 460 & 132.3 & 10.9 & 8.8 \\
\hline $7 / 15 / 1999$ & 23:00:40 & 24.86 & 461 & 127.5 & 10.6 & 8.8 \\
\hline 7/15/1999 & $23: 30: 40$ & 24.77 & 461 & 122.7 & 10.2 & 8.7 \\
\hline $7 / 16 / 1999$ & 0:00:40 & 24.73 & 461 & 119.6 & 9.9 & 8.7 \\
\hline 7/16/1999 & 0:30:40 & 24.69 & 460 & 113.7 & 9.4 & 8.7 \\
\hline $7 / 16 / 1999$ & 1:00:40 & 24.64 & 461 & 110.7 & 9.2 & 8.7 \\
\hline $7 / 16 / 1999$ & 1:30:40 & 24.58 & 461 & 107.2 & 8.9 & 8.7 \\
\hline $7 / 16 / 1999$ & 2:00:40 & 24.45 & 462 & 102.2 & 8.5 & 8.7 \\
\hline $7 / 16 / 1999$ & 2:30:40 & 24.35 & 463 & 98.3 & 8.2 & 8.7 \\
\hline 7/16/1999 & 3:00:40 & 24.26 & 464 & 94.3 & 7.9 & 8.6 \\
\hline $7 / 16 / 1999$ & $3: 30: 40$ & 24.17 & 464 & 90.5 & 7.6 & 8.6 \\
\hline 7/16/1999 & 4:00:40 & 24.06 & 465 & 86.2 & 7.2 & 8.6 \\
\hline $7 / 16 / 1999$ & 4:30:40 & 23.99 & 466 & 82.9 & 7.0 & 8.6 \\
\hline 7/16/1999 & 5:00:40 & 23.93 & 468 & 79.7 & 6.7 & 8.5 \\
\hline 7/16/1999 & $5: 30: 40$ & 23.85 & 467 & 76.1 & 6.4 & 8.5 \\
\hline 7/16/1999 & 6:00:40 & 23.75 & 467 & 73.1 & 6.2 & 8.5 \\
\hline 7/16/1999 & $6: 30: 40$ & 23.61 & 469 & 71 & 6.0 & 8.4 \\
\hline $7 / 16 / 1999$ & 7:00:40 & 23.5 & 469 & 70.3 & 6.0 & 8.4 \\
\hline 7/16/1999 & 7:30:40 & 23.46 & 469 & 71 & 6.0 & 8.4 \\
\hline 7/16/1999 & 8:00:40 & 23.4 & 470 & 71.8 & 6.1 & 8.4 \\
\hline $7 / 16 / 1999$ & 8:30:40 & 23.38 & 470 & 73.1 & 6.2 & 8.4 \\
\hline $7 / 16 / 1999$ & 9:00:40 & 23.42 & 469 & 75.4 & 6.4 & 8.4 \\
\hline 7/16/1999 & $9: 30: 40$ & 23.6 & 469 & 81 & 6.9 & 8.4 \\
\hline 7/16/1999 & 10:00:40 & 23.79 & 469 & 86.5 & 7.3 & 8.4 \\
\hline $7 / 16 / 1999$ & 10:30:40 & 23.95 & 468 & 90.6 & 7.6 & 8.4 \\
\hline 7/16/1999 & 11:00:40 & 24.2 & 467 & 96.8 & 8.1 & 8.5 \\
\hline 7/16/1999 & 11:30:40 & 24.64 & 466 & 105 & 8.7 & 8.5 \\
\hline 7/16/1999 & 12:00:40 & 24.78 & 465 & 109.7 & 9.1 & 8.5 \\
\hline 7/16/1999 & $12: 30: 40$ & 25.04 & 465 & 116.4 & 9.6 & 8.6 \\
\hline 7/16/1999 & 13:00:40 & 25.35 & 464 & 123.8 & 10.2 & 8.6 \\
\hline 7/16/1999 & $13: 30: 40$ & 25.51 & 464 & 129.4 & 10.6 & 8.6 \\
\hline
\end{tabular}


Appendix Continued

\begin{tabular}{|c|c|c|c|c|c|c|}
\hline Date & Time & $\begin{array}{c}\text { Water } \\
\text { temperature } \\
\text { (C) }\end{array}$ & $\begin{array}{c}\text { SC } \\
(\mu \mathrm{S} / \mathrm{cm})\end{array}$ & $\begin{array}{c}\text { DOsat } \\
\text { (percent) }\end{array}$ & $\begin{array}{c}\mathrm{DO} \\
\text { (mg/L) }\end{array}$ & $\begin{array}{c}\mathrm{pH} \\
\text { (standard } \\
\text { units) }\end{array}$ \\
\hline $7 / 16 / 1999$ & $14: 00: 40$ & 25.76 & 462 & 135 & 11.0 & 8.6 \\
\hline $7 / 16 / 1999$ & $14: 30: 40$ & 25.98 & 461 & 141.2 & 11.5 & 8.7 \\
\hline $7 / 16 / 1999$ & 15:00:40 & 26.04 & 461 & 143.7 & 11.6 & 8.7 \\
\hline 7/16/1999 & $15: 30: 40$ & 26.08 & 459 & 148 & 12.0 & 8.8 \\
\hline $7 / 16 / 1999$ & $16: 00: 40$ & 26.34 & 457 & 153.9 & 12.4 & 8.8 \\
\hline $7 / 16 / 1999$ & $16: 30: 40$ & 26.53 & 457 & 160.4 & 12.9 & 8.8 \\
\hline $7 / 16 / 1999$ & 17:00:40 & 26.64 & 456 & 165.1 & 13.2 & 8.8 \\
\hline 7/16/1999 & $17: 30: 40$ & 26.72 & 455 & 168 & $13.4^{\mathbf{a}}$ & 8.8 \\
\hline $7 / 16 / 1999$ & 18:00:40 & 26.77 & 453 & 167.8 & 13.4 & 8.8 \\
\hline 7/16/1999 & $18: 30: 40$ & 26.76 & 451 & 167.8 & 13.4 & 8.8 \\
\hline $7 / 16 / 1999$ & 19:00:40 & 26.72 & 450 & 163.1 & 13.1 & 8.8 \\
\hline $7 / 16 / 1999$ & $19: 30: 40$ & 26.65 & 448 & 160.6 & 12.9 & 8.8 \\
\hline $7 / 16 / 1999$ & 20:00:40 & 26.54 & 447 & 154.1 & 12.4 & 8.8 \\
\hline $7 / 16 / 1999$ & $20: 30: 40$ & 26.41 & 447 & 148.1 & 11.9 & 8.8 \\
\hline $7 / 16 / 1999$ & 21:00:40 & 26.28 & 447 & 143.8 & 11.6 & 8.8 \\
\hline $7 / 16 / 1999$ & $21: 30: 40$ & 26.17 & 447 & 139.3 & 11.3 & 8.8 \\
\hline 7/16/1999 & $22: 00: 40$ & 26.09 & 447 & 136 & 11.0 & 8.8 \\
\hline $7 / 16 / 1999$ & $22: 30: 40$ & 26.01 & 447 & 132.1 & 10.7 & 8.8 \\
\hline $7 / 16 / 1999$ & 23:00:40 & 25.94 & 448 & 128.4 & 10.4 & 8.8 \\
\hline $7 / 16 / 1999$ & $23: 30: 40$ & 25.87 & 448 & 124 & 10.1 & 8.8 \\
\hline $7 / 17 / 1999$ & 0:00:40 & 25.78 & 449 & 119.9 & 9.8 & 8.8 \\
\hline $7 / 17 / 1999$ & $0: 30: 40$ & 25.64 & 450 & 114.6 & 9.4 & 8.7 \\
\hline $7 / 17 / 1999$ & 1:00:40 & 25.51 & 452 & 109.9 & 9.0 & 8.7 \\
\hline 7/17/1999 & $1: 30: 40$ & 25.4 & 453 & 104.5 & 8.6 & 8.7 \\
\hline 7/17/1999 & 2:00:40 & 25.3 & 454 & 99.7 & 8.2 & 8.7 \\
\hline 7/17/1999 & $2: 30: 40$ & 25.21 & 455 & 95.6 & 7.9 & 8.7 \\
\hline 7/17/1999 & 3:00:40 & 25.12 & 456 & 91.8 & 7.6 & 8.6 \\
\hline 7/17/1999 & $3: 30: 40$ & 25.04 & 457 & 87.5 & 7.2 & 8.6 \\
\hline $7 / 17 / 1999$ & 4:00:40 & 24.97 & 459 & 83.1 & 6.9 & 8.6 \\
\hline $7 / 17 / 1999$ & $4: 30: 40$ & 24.87 & 460 & 79.6 & 6.6 & 8.5 \\
\hline $7 / 17 / 1999$ & $5: 00: 40$ & 24.71 & 462 & 75.4 & 6.3 & 8.5 \\
\hline $7 / 17 / 1999$ & $5: 30: 40$ & 24.57 & 464 & 72.5 & 6.0 & 8.5 \\
\hline 7/17/1999 & 6:00:40 & 24.45 & 464 & 69.6 & 5.8 & 8.4 \\
\hline $7 / 17 / 1999$ & $6: 30: 40$ & 24.35 & 465 & 67.7 & 5.7 & 8.4 \\
\hline 7/17/1999 & 7:00:40 & 24.31 & 466 & 66.8 & 5.6 & 8.4 \\
\hline $7 / 17 / 1999$ & $7: 30: 40$ & 24.27 & 467 & 68 & 5.7 & 8.4 \\
\hline 7/17/1999 & 8:00:40 & 24.24 & 466 & 69.2 & 5.8 & 8.4 \\
\hline $7 / 17 / 1999$ & $8: 30: 40$ & 24.23 & 466 & 71.3 & 6.0 & 8.4 \\
\hline 7/17/1999 & 9:00:40 & 24.22 & 467 & 74 & 6.2 & 8.4 \\
\hline 7/17/1999 & 9:30:40 & 24.31 & 467 & 79.8 & 6.7 & 8.5 \\
\hline 7/17/1999 & 10:00:40 & 24.46 & 466 & 92.7 & 7.7 & 8.6 \\
\hline 7/17/1999 & $10: 30: 40$ & 24.84 & 464 & 101 & 8.4 & 8.6 \\
\hline $7 / 17 / 1999$ & 11:00:40 & 24.99 & 464 & 104.4 & 8.6 & 8.6 \\
\hline $7 / 17 / 1999$ & 11:30:40 & 25.23 & 463 & 109.2 & 9.0 & 8.6 \\
\hline $7 / 17 / 1999$ & $12: 00: 40$ & 25.49 & 462 & 112.5 & 9.2 & 8.6 \\
\hline 7/17/1999 & $12: 30: 40$ & 25.68 & 462 & 120 & 9.8 & 8.6 \\
\hline 7/17/1999 & 13:00:40 & 25.94 & 461 & 126.5 & 10.3 & 8.6 \\
\hline 7/17/1999 & $13: 30: 40$ & 26.11 & 460 & 131.7 & 10.7 & 8.6 \\
\hline 7/17/1999 & 14:00:40 & 26.55 & 458 & 141.3 & 11.3 & 8.7 \\
\hline 7/17/1999 & $14: 30: 40$ & 26.75 & 458 & 143.6 & 11.5 & 8.7 \\
\hline
\end{tabular}


Water-Quality Synoptic Sampling, July 1999: North Fork Shenandoah River, Virginia

Appendix Continued

\begin{tabular}{|c|c|c|c|c|c|c|}
\hline Date & Time & $\begin{array}{c}\text { Water } \\
\text { temperature } \\
\text { (ㄷ) }\end{array}$ & $\begin{array}{c}\text { SC } \\
(\mu \mathbf{S} / \mathbf{c m})\end{array}$ & $\begin{array}{c}\text { DOsat } \\
\text { (percent) }\end{array}$ & $\begin{array}{c}\mathrm{DO} \\
(\mathrm{mg} / \mathrm{L})\end{array}$ & $\begin{array}{c}\text { pH } \\
\text { (standard } \\
\text { units) }\end{array}$ \\
\hline $7 / 17 / 1999$ & $15: 00: 40$ & 26.98 & 457 & 150.1 & 12.0 & 8.7 \\
\hline 7/17/1999 & $15: 30: 40$ & 27.22 & 456 & 155.2 & 12.3 & 8.7 \\
\hline 7/17/1999 & $16: 00: 40$ & 27.32 & 454 & 160.1 & 12.7 & 8.7 \\
\hline 7/17/1999 & $16: 30: 40$ & 27.4 & 438 & 156.3 & 12.4 & 8.7 \\
\hline $7 / 17 / 1999$ & 17:00:40 & 27.53 & 435 & 155.9 & 12.3 & 8.7 \\
\hline 7/17/1999 & $17: 30: 40$ & 27.58 & 431 & 157.3 & 12.4 & 8.8 \\
\hline 7/17/1999 & 18:00:40 & 27.5 & 429 & 154.3 & 12.2 & 8.8 \\
\hline 7/17/1999 & $18: 30: 40$ & 27.4 & 427 & 152 & 12.0 & 8.8 \\
\hline 7/17/1999 & 19:00:40 & 27.32 & 426 & 149.7 & 11.9 & 8.8 \\
\hline 7/17/1999 & $19: 30: 40$ & 27.19 & 424 & 146.8 & 11.7 & 8.8 \\
\hline $7 / 17 / 1999$ & 20:00:40 & 27.08 & 425 & 143.2 & 11.4 & 8.8 \\
\hline $7 / 17 / 1999$ & $20: 30: 40$ & 27.01 & 424 & 138.6 & 11.0 & 8.8 \\
\hline 7/17/1999 & 21:00:40 & 26.96 & 423 & 136.2 & 10.9 & 8.8 \\
\hline $7 / 17 / 1999$ & $21: 30: 40$ & 26.91 & 424 & 133.2 & 10.6 & 8.8 \\
\hline 7/17/1999 & $22: 00: 40$ & 26.84 & 423 & 129.5 & 10.3 & 8.7 \\
\hline 7/17/1999 & $22: 30: 40$ & 26.74 & 424 & 125.6 & 10.1 & 8.7 \\
\hline 7/17/1999 & 23:00:40 & 26.58 & 426 & 121.4 & 9.7 & 8.7 \\
\hline 7/17/1999 & $23: 30: 40$ & 26.46 & 428 & 115.9 & 9.3 & 8.7 \\
\hline $7 / 18 / 1999$ & 0:00:40 & 26.34 & 429 & 109.8 & 8.8 & 8.7 \\
\hline 7/18/1999 & $0: 30: 40$ & 26.23 & 431 & 104.6 & 8.4 & 8.7 \\
\hline $7 / 18 / 1999$ & 1:00:40 & 26.18 & 432 & 99.8 & 8.1 & 8.7 \\
\hline 7/18/1999 & 1:30:40 & 26.12 & 434 & 95.5 & 7.7 & 8.6 \\
\hline 7/18/1999 & 2:00:40 & 26.04 & 436 & 91.3 & 7.4 & 8.6 \\
\hline 7/18/1999 & $2: 30: 40$ & 25.9 & 438 & 86.4 & 7.0 & 8.6 \\
\hline 7/18/1999 & 3:00:40 & 25.76 & 441 & 82.5 & 6.7 & 8.6 \\
\hline $7 / 18 / 1999$ & $3: 30: 40$ & 25.67 & 443 & 79.3 & 6.5 & 8.5 \\
\hline 7/18/1999 & 4:00:40 & 25.56 & 445 & 75.1 & 6.1 & 8.5 \\
\hline $7 / 18 / 1999$ & 4:30:40 & 25.48 & 445 & 71.9 & 5.9 & 8.5 \\
\hline 7/18/1999 & 5:00:40 & 25.41 & 447 & 68.9 & 5.7 & 8.4 \\
\hline 7/18/1999 & $5: 30: 40$ & 25.33 & 448 & 65.6 & 5.4 & 8.4 \\
\hline 7/18/1999 & 6:00:40 & 25.22 & 448 & 63.2 & 5.2 & 8.4 \\
\hline 7/18/1999 & $6: 30: 40$ & 25.07 & 449 & 60.9 & 5.0 & 8.3 \\
\hline $7 / 18 / 1999$ & 7:00:40 & 24.98 & 449 & 60.1 & 5.0 & 8.3 \\
\hline 7/18/1999 & 7:30:40 & 24.9 & 453 & 61.4 & 5.1 & 8.3 \\
\hline $7 / 18 / 1999$ & 8:00:40 & 24.87 & 452 & 63.9 & 5.3 & 8.3 \\
\hline 7/18/1999 & $8: 30: 40$ & 24.89 & 451 & 66.2 & 5.5 & 8.3 \\
\hline 7/18/1999 & 9:00:40 & 24.94 & 453 & 69.3 & 5.7 & 8.3 \\
\hline 7/18/1999 & 9:30:40 & 24.99 & 452 & 73.3 & 6.1 & 8.3 \\
\hline 7/18/1999 & 10:00:40 & 25.04 & 454 & 79.2 & 6.5 & 8.3 \\
\hline $7 / 18 / 1999$ & 10:30:40 & 25.22 & 454 & 87.1 & 7.2 & 8.4 \\
\hline 7/18/1999 & 11:00:40 & 25.37 & 452 & 94.1 & 7.7 & 8.4 \\
\hline $7 / 18 / 1999$ & 11:30:40 & 25.6 & 458 & 100.5 & 8.2 & 8.4 \\
\hline 7/18/1999 & $12: 00: 40$ & 25.91 & 456 & 107.5 & 8.7 & 8.5 \\
\hline $7 / 18 / 1999$ & $12: 30: 40$ & 26.07 & 455 & 113.4 & 9.2 & 8.5 \\
\hline 7/18/1999 & 13:00:40 & 26.41 & 456 & 120.8 & 9.7 & 8.6 \\
\hline 7/18/1999 & $13: 30: 40$ & 26.59 & 455 & 126.7 & 10.2 & 8.6 \\
\hline $7 / 18 / 1999$ & 14:00:40 & 26.52 & 456 & 125.8 & 10.1 & 8.6 \\
\hline 7/18/1999 & $14: 30: 40$ & 26.61 & 451 & 133.1 & 10.7 & 8.6 \\
\hline 7/18/1999 & $15: 00: 40$ & 26.49 & 454 & 130.2 & 10.5 & 8.6 \\
\hline 7/18/1999 & $15: 30: 40$ & 26.55 & 453 & 132.1 & 10.6 & 8.6 \\
\hline
\end{tabular}


Appendix Continued

\begin{tabular}{|c|c|c|c|c|c|c|}
\hline Date & Time & $\begin{array}{c}\text { Water } \\
\text { temperature } \\
\text { ('C) }\end{array}$ & $\begin{array}{c}\text { SC } \\
(\mu \mathbf{S} / \mathbf{c m})\end{array}$ & $\begin{array}{c}\text { DOsat } \\
\text { (percent) }\end{array}$ & $\begin{array}{c}\text { DO } \\
\text { (mg/L) }\end{array}$ & $\begin{array}{c}\text { pH } \\
\text { (standard } \\
\text { units) }\end{array}$ \\
\hline 7/18/1999 & $16: 00: 40$ & 26.59 & 452 & 135.9 & 10.9 & 8.6 \\
\hline 7/18/1999 & $16: 30: 40$ & 26.66 & 450 & 142.5 & 11.4 & 8.7 \\
\hline 7/18/1999 & 17:00:40 & 26.89 & 447 & 152.9 & 12.2 & 8.7 \\
\hline 7/18/1999 & $17: 30: 40$ & 26.96 & 447 & 156.2 & 12.4 & 8.7 \\
\hline 7/18/1999 & 18:00:40 & 26.98 & 445 & 156.2 & 12.4 & 8.8 \\
\hline 7/18/1999 & $18: 30: 40$ & 26.96 & 441 & 155.5 & 12.4 & 8.8 \\
\hline $7 / 18 / 1999$ & 19:00:40 & 26.91 & 443 & 152.5 & 12.2 & 8.8 \\
\hline $7 / 18 / 1999$ & $19: 30: 40$ & 26.87 & 445 & 148.1 & 11.8 & 8.8 \\
\hline 7/18/1999 & 20:00:40 & 26.83 & 444 & 145 & 11.6 & 8.8 \\
\hline 7/18/1999 & $20: 30: 40$ & 26.75 & 445 & 141.6 & 11.3 & 8.8 \\
\hline 7/18/1999 & 21:00:40 & 26.63 & 444 & 136.6 & 11.0 & 8.7 \\
\hline 7/18/1999 & $21: 30: 40$ & 26.53 & 451 & 132.2 & 10.6 & 8.7 \\
\hline 7/18/1999 & $22: 00: 40$ & 26.44 & 446 & 128.5 & 10.3 & 8.7 \\
\hline $7 / 18 / 1999$ & $22: 30: 40$ & 26.37 & 445 & 125.1 & 10.1 & 8.7 \\
\hline $7 / 18 / 1999$ & $23: 00: 40$ & 26.29 & 444 & 121.5 & 9.8 & 8.7 \\
\hline 7/18/1999 & $23: 30: 40$ & 26.25 & 445 & 117.8 & 9.5 & 8.7 \\
\hline $7 / 19 / 1999$ & 0:00:40 & 26.19 & 446 & 113.8 & 9.2 & 8.7 \\
\hline 7/19/1999 & 0:30:40 & 26.08 & 448 & 108.6 & 8.8 & 8.7 \\
\hline 7/19/1999 & 1:00:40 & 25.94 & 451 & 104.1 & 8.5 & 8.7 \\
\hline 7/19/1999 & $1: 30: 40$ & 25.82 & 453 & 99 & 8.1 & 8.6 \\
\hline $7 / 19 / 1999$ & 2:00:40 & 25.73 & 453 & 95.3 & 7.8 & 8.6 \\
\hline $7 / 19 / 1999$ & $2: 30: 40$ & 25.61 & 454 & 91.5 & 7.5 & 8.6 \\
\hline $7 / 19 / 1999$ & 3:00:40 & 25.54 & 453 & 87.8 & 7.2 & 8.6 \\
\hline $7 / 19 / 1999$ & $3: 30: 40$ & 25.48 & 454 & 83.7 & 6.9 & 8.6 \\
\hline 7/19/1999 & 4:00:40 & 25.42 & 454 & 79 & 6.5 & 8.5 \\
\hline 7/19/1999 & 4:30:40 & 25.34 & 454 & 76 & 6.2 & 8.5 \\
\hline 7/19/1999 & 5:00:40 & 25.24 & 458 & 72 & 5.9 & 8.5 \\
\hline $7 / 19 / 1999$ & $5: 30: 40$ & 25.07 & 457 & 68.7 & 5.7 & 8.4 \\
\hline $7 / 19 / 1999$ & $6: 00: 40$ & 24.95 & 459 & 65.9 & 5.4 & 8.4 \\
\hline 7/19/1999 & $6: 30: 40$ & 24.85 & 460 & 63.6 & 5.3 & 8.3 \\
\hline 7/19/1999 & 7:00:40 & 24.8 & 461 & 62.2 & 5.2 & 8.3 \\
\hline $7 / 19 / 1999$ & $7: 30: 40$ & 24.77 & 458 & 62.5 & 5.2 & 8.3 \\
\hline $7 / 19 / 1999$ & 8:00:40 & 24.73 & 458 & 63.3 & 5.3 & 8.3 \\
\hline 7/19/1999 & 8:30:40 & 24.72 & 459 & 65.8 & 5.5 & 8.3 \\
\hline 7/19/1999 & 9:00:40 & 24.65 & 459 & 68 & 5.7 & 8.3 \\
\hline $7 / 19 / 1999$ & 9:30:40 & 24.68 & 459 & 71.6 & 5.9 & 8.3 \\
\hline 7/19/1999 & 10:00:40 & 24.8 & 458 & 77.7 & 6.4 & 8.3 \\
\hline 7/19/1999 & $10: 30: 40$ & 25.03 & 457 & 85.3 & 7.0 & 8.4 \\
\hline 7/19/1999 & 11:00:40 & 25.31 & 455 & 93.2 & 7.7 & 8.4 \\
\hline $7 / 19 / 1999$ & 11:30:40 & 25.65 & 454 & 101 & 8.2 & 8.5 \\
\hline 7/19/1999 & 12:00:40 & 25.9 & 453 & 108.3 & 8.8 & 8.5 \\
\hline $7 / 19 / 1999$ & $12: 30: 40$ & 25.91 & 454 & 109.1 & 8.9 & 8.5 \\
\hline $7 / 19 / 1999$ & $13: 00: 40$ & 26.04 & 452 & 114.7 & 9.3 & 8.5 \\
\hline 7/19/1999 & $13: 30: 40$ & 26.24 & 463 & 132 & 10.7 & 8.6 \\
\hline 7/19/1999 & 14:00:40 & 26.41 & 463 & 141.9 & 11.4 & 8.7 \\
\hline 7/19/1999 & $14: 30: 40$ & 26.46 & 462 & 142.1 & 11.4 & 8.7 \\
\hline 7/19/1999 & $15: 00: 40$ & 26.81 & 460 & 159.5 & 12.7 & 8.8 \\
\hline 7/19/1999 & $15: 30: 40$ & 26.79 & 460 & 152.1 & 12.2 & 8.7 \\
\hline 7/19/1999 & $16: 00: 40$ & 26.78 & 460 & 148.8 & 11.9 & 8.7 \\
\hline $7 / 19 / 1999$ & $16: 30: 40$ & 27.01 & 457 & 159.8 & 12.7 & 8.8 \\
\hline
\end{tabular}


Water-Quality Synoptic Sampling, July 1999: North Fork Shenandoah River, Virginia

Appendix Continued

\begin{tabular}{|c|c|c|c|c|c|c|}
\hline Date & Time & $\begin{array}{c}\text { Water } \\
\text { temperature } \\
\text { ('C) }\end{array}$ & $\begin{array}{c}\text { SC } \\
(\mu \mathrm{S} / \mathbf{c m})\end{array}$ & $\begin{array}{c}\text { DOsat } \\
\text { (percent) }\end{array}$ & $\begin{array}{c}\text { DO } \\
\text { (mg/L) }\end{array}$ & $\begin{array}{c}\text { pH } \\
\text { (standard } \\
\text { units) }\end{array}$ \\
\hline $7 / 19 / 1999$ & $17: 00: 40$ & 27.11 & 456 & 162.9 & 12.9 & 8.8 \\
\hline 7/19/1999 & $17: 30: 40$ & 27.09 & 456 & 162.9 & 12.9 & 8.8 \\
\hline 7/19/1999 & 18:00:40 & 27.05 & 454 & 160.6 & 12.8 & 8.8 \\
\hline 7/19/1999 & $18: 30: 40$ & 27.1 & 453 & 163.2 & 13.0 & 8.8 \\
\hline 7/19/1999 & 19:00:40 & 27.13 & 452 & 161.6 & 12.8 & 8.8 \\
\hline $7 / 19 / 1999$ & $19: 30: 40$ & 27.05 & 452 & 154.3 & 12.3 & 8.8 \\
\hline $7 / 19 / 1999$ & 20:00:40 & 27.01 & 452 & 148.9 & 11.9 & 8.8 \\
\hline 7/19/1999 & $20: 30: 40$ & 26.94 & 453 & 144.3 & 11.5 & 8.7 \\
\hline $7 / 19 / 1999$ & 21:00:40 & 26.83 & 454 & 137.6 & 11.0 & 8.7 \\
\hline 7/19/1999 & $21: 30: 40$ & 26.76 & 453 & 134.3 & 10.7 & 8.7 \\
\hline $7 / 19 / 1999$ & $22: 00: 40$ & 26.68 & 453 & 129.3 & 10.4 & 8.7 \\
\hline $7 / 19 / 1999$ & $22: 30: 40$ & 26.61 & 453 & 127 & 10.2 & 8.7 \\
\hline $7 / 19 / 1999$ & 23:00:40 & 26.57 & 454 & 122 & 9.8 & 8.7 \\
\hline $7 / 19 / 1999$ & $23: 30: 40$ & 26.53 & 454 & 117.6 & 9.4 & 8.7 \\
\hline $7 / 20 / 1999$ & 0:00:40 & 26.46 & 455 & 113.2 & 9.1 & 8.7 \\
\hline 7/20/1999 & 0:30:40 & 26.34 & 456 & 108.5 & 8.7 & 8.7 \\
\hline 7/20/1999 & 1:00:40 & 26.22 & 458 & 104.1 & 8.4 & 8.6 \\
\hline $7 / 20 / 1999$ & 1:30:40 & 26.12 & 459 & 99 & 8.0 & 8.6 \\
\hline 7/20/1999 & 2:00:40 & 26.03 & 460 & 94.8 & 7.7 & 8.6 \\
\hline $7 / 20 / 1999$ & $2: 30: 40$ & 25.95 & 461 & 90.4 & 7.3 & 8.6 \\
\hline 7/20/1999 & 3:00:40 & 25.89 & 461 & 85.2 & 6.9 & 8.5 \\
\hline $7 / 20 / 1999$ & $3: 30: 40$ & 25.82 & 462 & 81.6 & 6.6 & 8.5 \\
\hline 7/20/1999 & 4:00:40 & 25.74 & 463 & 77.1 & 6.3 & 8.5 \\
\hline $7 / 20 / 1999$ & 4:30:40 & 25.63 & 465 & 73.9 & 6.0 & 8.4 \\
\hline $7 / 20 / 1999$ & 5:00:40 & 25.49 & 466 & 70.3 & 5.8 & 8.4 \\
\hline 7/20/1999 & $5: 30: 40$ & 25.39 & 467 & 67 & 5.5 & 8.4 \\
\hline $7 / 20 / 1999$ & 6:00:40 & 25.34 & 468 & 63.7 & 5.2 & 8.3 \\
\hline $7 / 20 / 1999$ & $6: 30: 40$ & 25.28 & 468 & 61.3 & 5.0 & 8.3 \\
\hline $7 / 20 / 1999$ & 7:00:40 & 25.23 & 468 & 60.4 & 5.0 & 8.3 \\
\hline $7 / 20 / 1999$ & 7:30:40 & 25.18 & 468 & 59.4 & 4.9 & 8.3 \\
\hline $7 / 20 / 1999$ & 8:00:40 & 25.09 & 470 & 57.6 & $4.8^{\mathbf{b}}$ & 8.2 \\
\hline $7 / 20 / 1999$ & 8:30:40 & 25.01 & 470 & 60.5 & 5.0 & 8.2 \\
\hline 7/20/1999 & 9:00:40 & 25.01 & 470 & 67.1 & 5.5 & 8.3 \\
\hline $7 / 20 / 1999$ & 9:30:40 & 25.03 & 470 & 71.5 & 5.9 & 8.3 \\
\hline $7 / 20 / 1999$ & 10:00:40 & 25.12 & 468 & 78.3 & 6.5 & 8.4 \\
\hline $7 / 20 / 1999$ & $10: 30: 40$ & 25.32 & 468 & 85 & 7.0 & 8.4 \\
\hline $7 / 20 / 1999$ & 11:00:40 & 25.56 & 467 & 95.4 & 7.8 & 8.5 \\
\hline 7/20/1999 & $11: 30: 40$ & 25.56 & 467 & 96.6 & 7.9 & 8.4 \\
\hline $7 / 20 / 1999$ & $12: 00: 40$ & 25.68 & 467 & 102.8 & 8.4 & 8.5 \\
\hline 7/20/1999 & $12: 30: 40$ & 25.54 & 467 & 95.9 & 7.8 & 8.5 \\
\hline 7/20/1999 & $13: 00: 40$ & 25.75 & 466 & 112 & 9.1 & 8.6 \\
\hline $7 / 20 / 1999$ & $13: 30: 40$ & 25.85 & 465 & 119.9 & 9.7 & 8.6 \\
\hline $7 / 20 / 1999$ & 14:00:40 & 25.96 & 465 & 125.7 & 10.2 & 8.6 \\
\hline 7/20/1999 & $14: 30: 40$ & 25.97 & 464 & 127.8 & 10.4 & 8.6 \\
\hline 7/20/1999 & 15:00:40 & 26.16 & 463 & 138.9 & 11.2 & 8.7 \\
\hline \multicolumn{7}{|c|}{ Site 37} \\
\hline $7 / 21 / 1999$ & $10: 42: 03$ & 26.8 & 430 & 72.9 & 5.8 & 8.6 \\
\hline 7/21/1999 & 11:00:40 & 26.93 & 431 & 76.1 & 6.1 & 8.6 \\
\hline $7 / 21 / 1999$ & 11:30:40 & 27.14 & 431 & 80.2 & 6.4 & 8.7 \\
\hline
\end{tabular}


Appendix Continued

\begin{tabular}{|c|c|c|c|c|c|c|}
\hline Date & Time & $\begin{array}{c}\text { Water } \\
\text { temperature } \\
\text { (ㄷ) }\end{array}$ & $\begin{array}{c}\text { SC } \\
(\mu \mathrm{S} / \mathbf{c m})\end{array}$ & $\begin{array}{c}\text { DOsat } \\
\text { (percent) }\end{array}$ & $\begin{array}{c}\mathrm{DO} \\
(\mathrm{mg} / \mathrm{L})\end{array}$ & $\begin{array}{c}\mathrm{pH} \\
\text { (standard } \\
\text { units) }\end{array}$ \\
\hline 7/21/1999 & $12: 00: 40$ & 27.43 & 430 & 86.5 & 6.8 & 8.7 \\
\hline $7 / 21 / 1999$ & $12: 30: 40$ & 27.72 & 429 & 92.7 & 7.3 & 8.7 \\
\hline $7 / 21 / 1999$ & 13:00:40 & 27.93 & 428 & 97.6 & 7.6 & 8.7 \\
\hline 7/21/1999 & $13: 30: 40$ & 28.09 & 427 & 102.3 & 8.0 & 8.7 \\
\hline $7 / 21 / 1999$ & 14:00:40 & 28.3 & 426 & 108.1 & 8.4 & 8.8 \\
\hline 7/21/1999 & $14: 30: 40$ & 28.54 & 425 & 114.3 & 8.9 & 8.8 \\
\hline $7 / 21 / 1999$ & $15: 00: 40$ & 28.73 & 424 & 118.4 & 9.1 & 8.8 \\
\hline $7 / 21 / 1999$ & $15: 30: 40$ & 28.8 & 424 & 119.8 & 9.2 & 8.8 \\
\hline $7 / 21 / 1999$ & $16: 00: 40$ & 28.81 & 422 & 120.8 & 9.3 & 8.8 \\
\hline 7/21/1999 & $16: 30: 40$ & 28.85 & 422 & 121.7 & 9.4 & 8.8 \\
\hline $7 / 21 / 1999$ & 17:00:40 & 28.81 & 420 & 121.1 & 9.3 & 8.8 \\
\hline $7 / 21 / 1999$ & $17: 30: 40$ & 28.7 & 413 & 117.8 & 9.1 & 8.9 \\
\hline $7 / 21 / 1999$ & 18:00:40 & 28.64 & 411 & 116.3 & 9.0 & 8.9 \\
\hline $7 / 21 / 1999$ & 18:30:40 & 28.64 & 413 & 115.7 & 8.9 & 8.9 \\
\hline $7 / 21 / 1999$ & 19:00:40 & 28.59 & 413 & 112.1 & 8.7 & 8.8 \\
\hline $7 / 21 / 1999$ & $19: 30: 40$ & 28.52 & 413 & 108.7 & 8.4 & 8.8 \\
\hline $7 / 21 / 1999$ & 20:00:40 & 28.34 & 409 & 103.6 & 8.1 & 8.8 \\
\hline $7 / 21 / 1999$ & $20: 30: 40$ & 28.1 & 408 & 97.3 & 7.6 & 8.8 \\
\hline $7 / 21 / 1999$ & 21:00:40 & 27.85 & 407 & 91.9 & 7.2 & 8.8 \\
\hline $7 / 21 / 1999$ & $21: 30: 40$ & 27.6 & 406 & 87.1 & 6.9 & 8.8 \\
\hline $7 / 21 / 1999$ & $22: 00: 40$ & 27.36 & 405 & 83.1 & 6.6 & 8.7 \\
\hline 7/21/1999 & $22: 30: 40$ & 27.24 & 409 & 80.3 & 6.4 & 8.7 \\
\hline $7 / 21 / 1999$ & 23:00:40 & 27.2 & 414 & 78.8 & 6.3 & 8.7 \\
\hline $7 / 21 / 1999$ & $23: 30: 40$ & 27.15 & 417 & 77.6 & 6.2 & 8.7 \\
\hline $7 / 22 / 1999$ & 0:00:40 & 27.07 & 420 & 75.8 & 6.0 & 8.7 \\
\hline $7 / 22 / 1999$ & $0: 30: 40$ & 26.97 & 422 & 73.3 & 5.8 & 8.7 \\
\hline $7 / 22 / 1999$ & 1:00:40 & 26.87 & 424 & 70.3 & 5.6 & 8.7 \\
\hline $7 / 22 / 1999$ & $1: 30: 40$ & 26.79 & 425 & 68.4 & 5.5 & 8.7 \\
\hline $7 / 22 / 1999$ & 2:00:40 & 26.73 & 425 & 67.3 & 5.4 & 8.6 \\
\hline $7 / 22 / 1999$ & $2: 30: 40$ & 26.68 & 426 & 66.8 & 5.4 & 8.6 \\
\hline $7 / 22 / 1999$ & 3:00:40 & 26.64 & 426 & 66.4 & 5.3 & 8.6 \\
\hline $7 / 22 / 1999$ & 3:30:40 & 26.61 & 426 & 66.3 & 5.3 & 8.6 \\
\hline $7 / 22 / 1999$ & 4:00:40 & 26.58 & 427 & 66.2 & 5.3 & 8.6 \\
\hline $7 / 22 / 1999$ & 4:30:40 & 26.56 & 427 & 65.8 & 5.3 & 8.6 \\
\hline $7 / 22 / 1999$ & 5:00:40 & 26.53 & 428 & 65.9 & 5.3 & 8.6 \\
\hline $7 / 22 / 1999$ & $5: 30: 40$ & 26.5 & 428 & 66.1 & 5.3 & 8.6 \\
\hline $7 / 22 / 1999$ & 6:00:40 & 26.48 & 428 & 66 & 5.3 & 8.6 \\
\hline $7 / 22 / 1999$ & 6:30:40 & 26.46 & 428 & 66.5 & 5.4 & 8.6 \\
\hline $7 / 22 / 1999$ & 7:00:40 & 26.45 & 429 & 66.6 & 5.4 & 8.6 \\
\hline $7 / 22 / 1999$ & 7:30:40 & 26.45 & 429 & 66.9 & 5.4 & 8.6 \\
\hline $7 / 22 / 1999$ & 8:00:40 & 26.46 & 429 & 67.5 & 5.4 & 8.6 \\
\hline $7 / 22 / 1999$ & $8: 30: 40$ & 26.48 & 429 & 68.1 & 5.5 & 8.6 \\
\hline $7 / 22 / 1999$ & 9:00:40 & 26.53 & 429 & 69.6 & 5.6 & 8.6 \\
\hline $7 / 22 / 1999$ & $9: 30: 40$ & 26.66 & 429 & 72 & 5.8 & 8.6 \\
\hline $7 / 22 / 1999$ & 10:00:40 & 26.91 & 429 & 75.7 & 6.0 & 8.6 \\
\hline $7 / 22 / 1999$ & $10: 30: 40$ & 27.18 & 428 & 79.8 & 6.3 & 8.6 \\
\hline $7 / 22 / 1999$ & 11:00:40 & 27.45 & 427 & 85.5 & 6.8 & 8.6 \\
\hline $7 / 22 / 1999$ & 11:30:40 & 27.73 & 427 & 91.8 & 7.2 & 8.7 \\
\hline $7 / 22 / 1999$ & $12: 00: 40$ & 28.17 & 425 & 99.6 & 7.8 & 8.7 \\
\hline $7 / 22 / 1999$ & $12: 30: 40$ & 28.54 & 424 & 106.1 & 8.2 & 8.7 \\
\hline
\end{tabular}


Water-Quality Synoptic Sampling, July 1999: North Fork Shenandoah River, Virginia

Appendix Continued

\begin{tabular}{|c|c|c|c|c|c|c|}
\hline Date & Time & $\begin{array}{c}\text { Water } \\
\text { temperature } \\
\text { ('C) }\end{array}$ & $\begin{array}{c}\text { SC } \\
(\mu \mathbf{S} / \mathbf{c m})\end{array}$ & $\begin{array}{c}\text { DOsat } \\
\text { (percent) }\end{array}$ & $\begin{array}{c}\mathrm{DO} \\
(\mathrm{mg} / \mathrm{L})\end{array}$ & $\begin{array}{c}\mathrm{pH} \\
\text { (standard } \\
\text { units) }\end{array}$ \\
\hline $7 / 22 / 1999$ & $13: 00: 40$ & 28.65 & 423 & 110 & 8.5 & 8.8 \\
\hline $7 / 22 / 1999$ & $13: 30: 40$ & 28.74 & 423 & 112 & 8.6 & 8.8 \\
\hline $7 / 22 / 1999$ & 14:00:40 & 28.84 & 423 & 113.5 & 8.8 & 8.8 \\
\hline $7 / 22 / 1999$ & $14: 30: 40$ & 29.03 & 421 & 116 & 8.9 & 8.8 \\
\hline $7 / 22 / 1999$ & $15: 00: 40$ & 29.13 & 422 & 114.4 & 8.8 & 8.8 \\
\hline $7 / 22 / 1999$ & $15: 30: 40$ & 29.28 & 422 & 113.7 & 8.7 & 8.8 \\
\hline $7 / 22 / 1999$ & $16: 00: 40$ & 29.21 & 422 & 112.9 & 8.6 & 8.8 \\
\hline $7 / 22 / 1999$ & $16: 30: 40$ & 29.18 & 422 & 110.1 & 8.4 & 8.8 \\
\hline $7 / 22 / 1999$ & $17: 00: 40$ & 29.2 & 422 & 109.5 & 8.4 & 8.8 \\
\hline $7 / 22 / 1999$ & $17: 30: 40$ & 29.22 & 422 & 108.7 & 8.3 & 8.8 \\
\hline $7 / 22 / 1999$ & 18:00:40 & 29.25 & 422 & 109.6 & 8.4 & 8.8 \\
\hline $7 / 22 / 1999$ & $18: 30: 40$ & 29.27 & 422 & 111.6 & 8.5 & 8.8 \\
\hline $7 / 22 / 1999$ & 19:00:40 & 29.23 & 422 & 112.5 & 8.6 & 8.8 \\
\hline $7 / 22 / 1999$ & $19: 30: 40$ & 29.1 & 422 & 111.6 & 8.6 & 8.8 \\
\hline $7 / 22 / 1999$ & 20:00:40 & 28.95 & 422 & 110 & 8.5 & 8.8 \\
\hline $7 / 22 / 1999$ & $20: 30: 40$ & 28.7 & 422 & 107.3 & 8.3 & 8.8 \\
\hline $7 / 22 / 1999$ & 21:00:40 & 28.43 & 422 & 103.7 & 8.1 & 8.8 \\
\hline $7 / 22 / 1999$ & $21: 30: 40$ & 28.15 & 423 & 99 & 7.7 & 8.8 \\
\hline $7 / 22 / 1999$ & $22: 00: 40$ & 27.94 & 425 & 94.2 & 7.4 & 8.8 \\
\hline $7 / 22 / 1999$ & $22: 30: 40$ & 27.75 & 425 & 89.8 & 7.1 & 8.7 \\
\hline $7 / 22 / 1999$ & $23: 00: 40$ & 27.59 & 426 & 85.3 & 6.7 & 8.7 \\
\hline $7 / 22 / 1999$ & $23: 30: 40$ & 27.45 & 427 & 79.7 & 6.3 & 8.7 \\
\hline $7 / 23 / 1999$ & 0:00:40 & 27.38 & 428 & 74.7 & 5.9 & 8.6 \\
\hline 7/23/1999 & 0:30:40 & 27.39 & 429 & 71 & 5.6 & 8.6 \\
\hline 7/23/1999 & 1:00:40 & 27.43 & 429 & 69.8 & 5.5 & 8.6 \\
\hline 7/23/1999 & $1: 30: 40$ & 27.48 & 429 & 67.9 & 5.4 & 8.6 \\
\hline $7 / 23 / 1999$ & 2:00:40 & 27.51 & 430 & 66.7 & 5.3 & 8.6 \\
\hline $7 / 23 / 1999$ & $2: 30: 40$ & 27.5 & 431 & 66.2 & 5.2 & 8.6 \\
\hline $7 / 23 / 1999$ & 3:00:40 & 27.46 & 431 & 65.9 & 5.2 & 8.6 \\
\hline $7 / 23 / 1999$ & $3: 30: 40$ & 27.4 & 432 & 65.9 & 5.2 & 8.5 \\
\hline $7 / 23 / 1999$ & 4:00:40 & 27.35 & 432 & 65.8 & 5.2 & 8.5 \\
\hline 7/23/1999 & 4:30:40 & 27.29 & 432 & 65.8 & 5.2 & 8.5 \\
\hline $7 / 23 / 1999$ & 5:00:40 & 27.22 & 432 & 65.7 & 5.2 & 8.5 \\
\hline $7 / 23 / 1999$ & $5: 30: 40$ & 27.15 & 433 & 65.3 & 5.2 & 8.5 \\
\hline 7/23/1999 & 6:00:40 & 27.08 & 433 & 65.3 & 5.2 & 8.5 \\
\hline $7 / 23 / 1999$ & $6: 30: 40$ & 27.01 & 433 & 65.2 & 5.2 & 8.5 \\
\hline $7 / 23 / 1999$ & 7:00:40 & 26.96 & 433 & 65.5 & 5.2 & 8.5 \\
\hline $7 / 23 / 1999$ & $7: 30: 40$ & 26.94 & 434 & 65.8 & 5.2 & 8.5 \\
\hline $7 / 23 / 1999$ & 8:00:40 & 26.93 & 434 & 66.1 & 5.3 & 8.5 \\
\hline $7 / 23 / 1999$ & 8:30:40 & 26.96 & 434 & 67.3 & 5.4 & 8.5 \\
\hline $7 / 23 / 1999$ & 9:00:40 & 27.03 & 434 & 67.7 & 5.4 & 8.5 \\
\hline $7 / 23 / 1999$ & 9:30:40 & 27.18 & 433 & 70.5 & 5.6 & 8.5 \\
\hline $7 / 23 / 1999$ & 10:00:40 & 27.39 & 433 & 75.5 & 6.0 & 8.5 \\
\hline $7 / 23 / 1999$ & $10: 30: 40$ & 27.59 & 433 & 77.5 & 6.1 & 8.5 \\
\hline $7 / 23 / 1999$ & 11:00:40 & 27.83 & 432 & 82 & 6.4 & 8.5 \\
\hline $7 / 23 / 1999$ & 11:30:40 & 28.17 & 432 & 87.3 & 6.8 & 8.5 \\
\hline $7 / 23 / 1999$ & 12:00:40 & 28.49 & 431 & 91.7 & 7.1 & 8.6 \\
\hline $7 / 23 / 1999$ & $12: 30: 40$ & 28.94 & 431 & 98.1 & 7.6 & 8.6 \\
\hline $7 / 23 / 1999$ & $13: 00: 40$ & 29.33 & 430 & 103.8 & 7.9 & 8.6 \\
\hline $7 / 23 / 1999$ & $13: 30: 40$ & 29.72 & 429 & 109.9 & 8.3 & 8.6 \\
\hline
\end{tabular}


Appendix Continued

\begin{tabular}{|c|c|c|c|c|c|c|}
\hline Date & Time & $\begin{array}{c}\text { Water } \\
\text { temperature } \\
\text { (ㄷ) }\end{array}$ & $\begin{array}{c}\text { SC } \\
(\mu \mathrm{S} / \mathbf{c m})\end{array}$ & $\begin{array}{c}\text { DOsat } \\
\text { (percent) }\end{array}$ & $\begin{array}{c}\mathrm{DO} \\
(\mathrm{mg} / \mathrm{L})\end{array}$ & $\begin{array}{c}\mathrm{pH} \\
\text { (standard } \\
\text { units) }\end{array}$ \\
\hline $7 / 23 / 1999$ & 14:00:40 & 30.08 & 429 & 115 & 8.7 & 8.7 \\
\hline $7 / 23 / 1999$ & $14: 30: 40$ & 30.22 & 428 & 117.4 & 8.8 & 8.7 \\
\hline $7 / 23 / 1999$ & $15: 00: 40$ & 30.48 & 428 & 121.1 & 9.1 & 8.7 \\
\hline 7/23/1999 & $15: 30: 40$ & 30.58 & 427 & 123.2 & 9.2 & 8.7 \\
\hline $7 / 23 / 1999$ & $16: 00: 40$ & 30.92 & 427 & 127.6 & 9.5 & 8.7 \\
\hline 7/23/1999 & $16: 30: 40$ & 31.03 & 427 & 129 & 9.6 & 8.7 \\
\hline $7 / 23 / 1999$ & 17:00:40 & 31.07 & 426 & 129.1 & 9.6 & 8.8 \\
\hline $7 / 23 / 1999$ & $17: 30: 40$ & 31.12 & 426 & 130.9 & 9.7 & 8.8 \\
\hline $7 / 23 / 1999$ & 18:00:40 & 31.07 & 426 & 130.2 & 9.7 & 8.8 \\
\hline 7/23/1999 & $18: 30: 40$ & 30.95 & 426 & 128.2 & 9.5 & 8.8 \\
\hline 7/23/1999 & 19:00:40 & 30.84 & 426 & 126.5 & 9.4 & 8.8 \\
\hline $7 / 23 / 1999$ & $19: 30: 40$ & 30.72 & 426 & 123.5 & 9.2 & 8.8 \\
\hline 7/23/1999 & 20:00:40 & 30.55 & 426 & 121.4 & 9.1 & 8.8 \\
\hline 7/23/1999 & $20: 30: 40$ & 30.33 & 426 & 118.7 & 8.9 & 8.8 \\
\hline 7/23/1999 & 21:00:40 & 30.04 & 426 & 115.5 & 8.7 & 8.8 \\
\hline $7 / 23 / 1999$ & $21: 30: 40$ & 29.82 & 426 & 111.3 & 8.4 & 8.7 \\
\hline 7/23/1999 & 22:00:40 & 29.64 & 427 & 105.2 & 8.0 & 8.7 \\
\hline 7/23/1999 & $22: 30: 40$ & 29.44 & 427 & 98.4 & 7.5 & 8.7 \\
\hline $7 / 23 / 1999$ & 23:00:40 & 29.26 & 428 & 92.9 & 7.1 & 8.7 \\
\hline $7 / 23 / 1999$ & $23: 30: 40$ & 29.08 & 429 & 85.8 & 6.6 & 8.7 \\
\hline $7 / 24 / 1999$ & 0:00:40 & 28.91 & 430 & 82.2 & 6.3 & 8.6 \\
\hline 7/24/1999 & $0: 30: 40$ & 28.73 & 431 & 77.3 & 6.0 & 8.6 \\
\hline 7/24/1999 & 1:00:40 & 28.56 & 432 & 72.3 & 5.6 & 8.6 \\
\hline $7 / 24 / 1999$ & $1: 30: 40$ & 28.38 & 432 & 68.9 & 5.4 & 8.6 \\
\hline $7 / 24 / 1999$ & 2:00:40 & 28.22 & 433 & 64.2 & 5.0 & 8.5 \\
\hline $7 / 24 / 1999$ & $2: 30: 40$ & 28.05 & 434 & 63.2 & 4.9 & 8.5 \\
\hline $7 / 24 / 1999$ & 3:00:40 & 27.88 & 434 & 62.1 & 4.9 & 8.5 \\
\hline $7 / 24 / 1999$ & 3:30:40 & 27.7 & 435 & 61.3 & 4.8 & 8.5 \\
\hline $7 / 24 / 1999$ & 4:00:40 & 27.54 & 435 & 61.2 & 4.8 & 8.4 \\
\hline $7 / 24 / 1999$ & $4: 30: 40$ & 27.4 & 435 & 61.6 & 4.9 & 8.4 \\
\hline $7 / 24 / 1999$ & 5:00:40 & 27.29 & 435 & 61.5 & 4.9 & 8.4 \\
\hline $7 / 24 / 1999$ & $5: 30: 40$ & 27.2 & 435 & 61.3 & 4.9 & 8.4 \\
\hline 7/24/1999 & 6:00:40 & 27.12 & 435 & 61.2 & 4.9 & 8.4 \\
\hline $7 / 24 / 1999$ & $6: 30: 40$ & 27.07 & 434 & 60.7 & 4.8 & 8.4 \\
\hline $7 / 24 / 1999$ & 7:00:40 & 27.01 & 435 & 61.1 & 4.9 & 8.4 \\
\hline $7 / 24 / 1999$ & $7: 30: 40$ & 26.99 & 435 & 61.6 & 4.9 & 8.4 \\
\hline $7 / 24 / 1999$ & 8:00:40 & 26.99 & 434 & 62.6 & 5.0 & 8.4 \\
\hline 7/24/1999 & 8:30:40 & 27.01 & 435 & 63.8 & 5.1 & 8.4 \\
\hline $7 / 24 / 1999$ & 9:00:40 & 27.08 & 434 & 65.7 & 5.2 & 8.4 \\
\hline $7 / 24 / 1999$ & $9: 30: 40$ & 27.21 & 434 & 67.7 & 5.4 & 8.4 \\
\hline $7 / 24 / 1999$ & 10:00:40 & 27.37 & 434 & 70.9 & 5.6 & 8.4 \\
\hline 7/24/1999 & $10: 30: 40$ & 27.52 & 434 & 72 & 5.7 & 8.4 \\
\hline 7/24/1999 & 11:00:40 & 27.73 & 433 & 76.2 & 6.0 & 8.4 \\
\hline $7 / 24 / 1999$ & $11: 30: 40$ & 27.85 & 433 & 78.7 & 6.2 & 8.4 \\
\hline $7 / 24 / 1999$ & $12: 00: 40$ & 27.97 & 432 & 82.9 & 6.5 & 8.5 \\
\hline $7 / 24 / 1999$ & $12: 30: 40$ & 28.08 & 432 & 87.2 & 6.8 & 8.5 \\
\hline 7/24/1999 & 13:00:40 & 28.19 & 431 & 91.2 & 7.1 & 8.5 \\
\hline 7/24/1999 & $13: 30: 40$ & 28.48 & 430 & 98.9 & 7.7 & 8.5 \\
\hline $7 / 24 / 1999$ & 14:00:40 & 28.96 & 428 & 106.8 & 8.2 & 8.6 \\
\hline $7 / 24 / 1999$ & $14: 30: 40$ & 29.19 & 427 & 111.1 & 8.5 & 8.6 \\
\hline
\end{tabular}


Water-Quality Synoptic Sampling, July 1999: North Fork Shenandoah River, Virginia

Appendix Continued

\begin{tabular}{|c|c|c|c|c|c|c|}
\hline Date & Time & $\begin{array}{c}\text { Water } \\
\text { temperature } \\
\text { ('C) }\end{array}$ & $\begin{array}{c}\text { SC } \\
(\mu \mathrm{S} / \mathbf{c m})\end{array}$ & $\begin{array}{c}\text { D0sat } \\
\text { (percent) }\end{array}$ & $\begin{array}{c}\text { DO } \\
\text { (mg/L) }\end{array}$ & $\begin{array}{c}\text { pH } \\
\text { (standard } \\
\text { units) }\end{array}$ \\
\hline $7 / 24 / 1999$ & $15: 00: 40$ & 29.35 & 427 & 113.3 & 8.7 & 8.6 \\
\hline $7 / 24 / 1999$ & $15: 30: 40$ & 29.48 & 427 & 114.8 & 8.8 & 8.7 \\
\hline $7 / 24 / 1999$ & 16:00:40 & 29.61 & 426 & 116.5 & 8.9 & 8.7 \\
\hline $7 / 24 / 1999$ & $16: 30: 40$ & 29.7 & 426 & 117.7 & 8.9 & 8.7 \\
\hline $7 / 24 / 1999$ & 17:00:40 & 29.76 & 426 & 118.8 & 9.0 & 8.7 \\
\hline $7 / 24 / 1999$ & $17: 30: 40$ & 29.78 & 425 & 121.1 & 9.2 & 8.7 \\
\hline 7/24/1999 & 18:00:40 & 29.83 & 425 & 122.5 & 9.3 & 8.7 \\
\hline $7 / 24 / 1999$ & $18: 30: 40$ & 29.86 & 425 & 123.1 & 9.3 & 8.7 \\
\hline $7 / 24 / 1999$ & 19:00:40 & 29.85 & 424 & 122.9 & 9.3 & 8.7 \\
\hline $7 / 24 / 1999$ & $19: 30: 40$ & 29.76 & 425 & 120.1 & 9.1 & 8.7 \\
\hline $7 / 24 / 1999$ & 20:00:40 & 29.65 & 425 & 116.9 & 8.9 & 8.7 \\
\hline $7 / 24 / 1999$ & $20: 30: 40$ & 29.52 & 425 & 112.2 & 8.5 & 8.7 \\
\hline $7 / 24 / 1999$ & 21:00:40 & 29.37 & 425 & 109 & 8.3 & 8.7 \\
\hline $7 / 24 / 1999$ & $21: 30: 40$ & 29.21 & 425 & 104.4 & 8.0 & 8.7 \\
\hline $7 / 24 / 1999$ & $22: 00: 40$ & 28.97 & 426 & 98.6 & 7.6 & 8.7 \\
\hline $7 / 24 / 1999$ & $22: 30: 40$ & 28.71 & 427 & 92.7 & 7.2 & 8.7 \\
\hline $7 / 24 / 1999$ & 23:00:40 & 28.43 & 428 & 86.2 & 6.7 & 8.6 \\
\hline $7 / 24 / 1999$ & $23: 30: 40$ & 28.21 & 429 & 81.3 & 6.3 & 8.6 \\
\hline $7 / 25 / 1999$ & 0:00:40 & 28.02 & 430 & 76.4 & 6.0 & 8.6 \\
\hline $7 / 25 / 1999$ & $0: 30: 40$ & 27.9 & 431 & 73.2 & 5.7 & 8.5 \\
\hline $7 / 25 / 1999$ & 1:00:40 & 27.79 & 431 & 70.8 & 5.6 & 8.5 \\
\hline $7 / 25 / 1999$ & $1: 30: 40$ & 27.7 & 432 & 67.8 & 5.3 & 8.5 \\
\hline $7 / 25 / 1999$ & 2:00:40 & 27.61 & 432 & 65.5 & 5.2 & 8.5 \\
\hline $7 / 25 / 1999$ & 2:30:40 & 27.55 & 432 & 63.7 & 5.0 & 8.5 \\
\hline $7 / 25 / 1999$ & 3:00:40 & 27.48 & 433 & 62.5 & 4.9 & 8.4 \\
\hline $7 / 25 / 1999$ & 3:30:40 & 27.41 & 433 & 61.9 & 4.9 & 8.4 \\
\hline $7 / 25 / 1999$ & 4:00:40 & 27.36 & 434 & 61.3 & 4.9 & 8.4 \\
\hline $7 / 25 / 1999$ & 4:30:40 & 27.3 & 434 & 61 & 4.8 & 8.4 \\
\hline $7 / 25 / 1999$ & 5:00:40 & 27.24 & 434 & 61.2 & 4.9 & 8.4 \\
\hline $7 / 25 / 1999$ & $5: 30: 40$ & 27.18 & 434 & 61.1 & 4.9 & 8.4 \\
\hline $7 / 25 / 1999$ & 6:00:40 & 27.12 & 434 & 61.1 & 4.9 & 8.4 \\
\hline $7 / 25 / 1999$ & $6: 30: 40$ & 27.06 & 434 & 61.7 & 4.9 & 8.4 \\
\hline $7 / 25 / 1999$ & 7:00:40 & 27 & 434 & 62 & 4.9 & 8.4 \\
\hline $7 / 25 / 1999$ & 7:30:40 & 26.96 & 434 & 62.4 & 5.0 & 8.4 \\
\hline $7 / 25 / 1999$ & 8:00:40 & 26.95 & 434 & 62.5 & 5.0 & 8.4 \\
\hline $7 / 25 / 1999$ & 8:30:40 & 26.99 & 434 & 64.4 & 5.1 & 8.4 \\
\hline $7 / 25 / 1999$ & 9:00:40 & 27.03 & 434 & 65 & 5.2 & 8.4 \\
\hline $7 / 25 / 1999$ & $9: 30: 40$ & 27.18 & 434 & 67.2 & 5.3 & 8.4 \\
\hline $7 / 25 / 1999$ & 10:00:40 & 27.41 & 434 & 72.5 & 5.7 & 8.4 \\
\hline $7 / 25 / 1999$ & 10:30:40 & 27.57 & 434 & 74.9 & 5.9 & 8.4 \\
\hline $7 / 25 / 1999$ & 11:00:40 & 27.77 & 434 & 78 & 6.1 & 8.4 \\
\hline $7 / 25 / 1999$ & 11:30:40 & 28.08 & 434 & 82.9 & 6.5 & 8.5 \\
\hline $7 / 25 / 1999$ & $12: 00: 40$ & 28.38 & 433 & 86.8 & 6.8 & 8.5 \\
\hline $7 / 25 / 1999$ & $12: 30: 40$ & 28.56 & 433 & 90.3 & 7.0 & 8.5 \\
\hline $7 / 25 / 1999$ & 13:00:40 & 28.85 & 432 & 96.4 & 7.4 & 8.5 \\
\hline $7 / 25 / 1999$ & $13: 30: 40$ & 29.11 & 430 & 103.5 & 7.9 & 8.6 \\
\hline $7 / 25 / 1999$ & 14:00:40 & 29.52 & 430 & 110.4 & 8.4 & 8.6 \\
\hline $7 / 25 / 1999$ & $14: 30: 40$ & 29.8 & 429 & 115.8 & 8.8 & 8.6 \\
\hline $7 / 25 / 1999$ & 15:00:40 & 29.77 & 428 & 116.7 & 8.9 & 8.7 \\
\hline $7 / 25 / 1999$ & $15: 30: 40$ & 29.9 & 428 & 118.5 & 9.0 & 8.7 \\
\hline
\end{tabular}


Appendix Continued

\begin{tabular}{|c|c|c|c|c|c|c|}
\hline Date & Time & $\begin{array}{c}\text { Water } \\
\text { temperature } \\
\text { (ㄷ) }\end{array}$ & $\begin{array}{c}\text { SC } \\
(\mu \mathbf{S} / \mathbf{c m})\end{array}$ & $\begin{array}{c}\text { DOsat } \\
\text { (percent) }\end{array}$ & $\begin{array}{c}\text { DO } \\
\text { (mg/L) }\end{array}$ & $\begin{array}{c}\text { pH } \\
\text { (standard } \\
\text { units) }\end{array}$ \\
\hline $7 / 25 / 1999$ & $16: 00: 40$ & 30 & 428 & 121.4 & 9.2 & 8.7 \\
\hline $7 / 25 / 1999$ & $16: 30: 40$ & 30.12 & 427 & 124.9 & 9.4 & 8.7 \\
\hline $7 / 25 / 1999$ & 17:00:40 & 30.16 & 427 & 125.6 & 9.5 & 8.7 \\
\hline $7 / 25 / 1999$ & $17: 30: 40$ & 30.14 & 426 & 125.5 & 9.5 & 8.7 \\
\hline $7 / 25 / 1999$ & 18:00:40 & 30.14 & 426 & 127.2 & 9.6 & 8.7 \\
\hline $7 / 25 / 1999$ & $18: 30: 40$ & 30.11 & 426 & 127.1 & 9.6 & 8.7 \\
\hline $7 / 25 / 1999$ & 19:00:40 & 30.12 & 426 & 127.1 & 9.6 & 8.8 \\
\hline $7 / 25 / 1999$ & $19: 30: 40$ & 30.11 & 425 & 127 & 9.6 & 8.8 \\
\hline $7 / 25 / 1999$ & 20:00:40 & 30.12 & 425 & 127.4 & 9.6 & 8.8 \\
\hline $7 / 25 / 1999$ & $20: 30: 40$ & 30.07 & 425 & 126.7 & 9.6 & 8.8 \\
\hline $7 / 25 / 1999$ & 21:00:40 & 29.91 & 425 & 124.3 & 9.4 & 8.8 \\
\hline $7 / 25 / 1999$ & $21: 30: 40$ & 29.68 & 426 & 120.1 & 9.1 & 8.8 \\
\hline $7 / 25 / 1999$ & $22: 00: 40$ & 29.45 & 426 & 114.2 & 8.7 & 8.8 \\
\hline $7 / 25 / 1999$ & $22: 30: 40$ & 29.23 & 427 & 108.7 & 8.3 & 8.7 \\
\hline $7 / 25 / 1999$ & $23: 00: 40$ & 28.99 & 428 & 102.7 & 7.9 & 8.7 \\
\hline $7 / 25 / 1999$ & $23: 30: 40$ & 28.77 & 429 & 96.1 & 7.4 & 8.7 \\
\hline $7 / 26 / 1999$ & 0:00:40 & 28.57 & 430 & 90.1 & 7.0 & 8.7 \\
\hline $7 / 26 / 1999$ & 0:30:40 & 28.38 & 431 & 84.2 & 6.5 & 8.6 \\
\hline $7 / 26 / 1999$ & 1:00:40 & 28.21 & 433 & 78.1 & 6.1 & 8.6 \\
\hline $7 / 26 / 1999$ & $1: 30: 40$ & 28.07 & 434 & 72.7 & 5.7 & 8.6 \\
\hline $7 / 26 / 1999$ & 2:00:40 & 27.94 & 435 & 68.2 & 5.3 & 8.5 \\
\hline $7 / 26 / 1999$ & $2: 30: 40$ & 27.84 & 436 & 64.6 & 5.1 & 8.5 \\
\hline $7 / 26 / 1999$ & 3:00:40 & 27.73 & 437 & 62.8 & 4.9 & 8.5 \\
\hline $7 / 26 / 1999$ & $3: 30: 40$ & 27.61 & 437 & 61.6 & 4.9 & 8.5 \\
\hline $7 / 26 / 1999$ & 4:00:40 & 27.5 & 438 & 60.7 & 4.8 & 8.5 \\
\hline $7 / 26 / 1999$ & 4:30:40 & 27.38 & 438 & 60.5 & 4.8 & 8.4 \\
\hline $7 / 26 / 1999$ & 5:00:40 & 27.27 & 440 & 60.3 & 4.8 & 8.4 \\
\hline $7 / 26 / 1999$ & $5: 30: 40$ & 27.17 & 438 & 60.1 & 4.8 & 8.4 \\
\hline $7 / 26 / 1999$ & $6: 00: 40$ & 27.07 & 438 & 60 & 4.8 & 8.4 \\
\hline $7 / 26 / 1999$ & $6: 30: 40$ & 26.98 & 438 & 59.7 & 4.8 & 8.4 \\
\hline $7 / 26 / 1999$ & 7:00:40 & 26.91 & 438 & 59.4 & $4.7^{\mathbf{b}}$ & 8.4 \\
\hline $7 / 26 / 1999$ & $7: 30: 40$ & 26.84 & 438 & 60.7 & 4.8 & 8.4 \\
\hline $7 / 26 / 1999$ & 8:00:40 & 26.79 & 437 & 61.5 & 4.9 & 8.4 \\
\hline $7 / 26 / 1999$ & $8: 30: 40$ & 26.79 & 437 & 62.4 & 5.0 & 8.4 \\
\hline 7/26/1999 & 9:00:40 & 26.79 & 437 & 62.8 & 5.0 & 8.4 \\
\hline $7 / 26 / 1999$ & 9:30:40 & 26.83 & 437 & 63.4 & 5.1 & 8.4 \\
\hline $7 / 26 / 1999$ & 10:00:40 & 26.85 & 437 & 64.5 & 5.2 & 8.4 \\
\hline $7 / 26 / 1999$ & $10: 30: 40$ & 26.88 & 437 & 66.8 & 5.3 & 8.4 \\
\hline $7 / 26 / 1999$ & 11:00:40 & 27.07 & 437 & 70.5 & 5.6 & 8.4 \\
\hline $7 / 26 / 1999$ & 11:30:40 & 27.29 & 437 & 74.8 & 5.9 & 8.4 \\
\hline $7 / 26 / 1999$ & $12: 00: 40$ & 27.57 & 436 & 79.1 & 6.2 & 8.4 \\
\hline $7 / 26 / 1999$ & $12: 30: 40$ & 27.81 & 436 & 83.2 & 6.5 & 8.5 \\
\hline $7 / 26 / 1999$ & $13: 00: 40$ & 28.07 & 435 & 88.1 & 6.9 & 8.5 \\
\hline $7 / 26 / 1999$ & $13: 30: 40$ & 28.35 & 434 & 94.4 & 7.3 & 8.5 \\
\hline 7/26/1999 & $14: 00: 40$ & 28.52 & 433 & 99.3 & 7.7 & 8.6 \\
\hline $7 / 26 / 1999$ & $14: 30: 40$ & 28.73 & 432 & 105.1 & 8.1 & 8.6 \\
\hline $7 / 26 / 1999$ & $15: 00: 40$ & 29 & 431 & 109.3 & 8.4 & 8.6 \\
\hline $7 / 26 / 1999$ & $15: 30: 40$ & 29.24 & 430 & 115.3 & 8.8 & 8.6 \\
\hline $7 / 26 / 1999$ & $16: 00: 40$ & 29.5 & 429 & 119.1 & 9.1 & 8.7 \\
\hline $7 / 26 / 1999$ & $16: 30: 40$ & 29.74 & 428 & 124 & 9.4 & 8.7 \\
\hline
\end{tabular}


Water-Quality Synoptic Sampling, July 1999: North Fork Shenandoah River, Virginia

Appendix Continued

\begin{tabular}{|c|c|c|c|c|c|c|}
\hline Date & Time & $\begin{array}{c}\text { Water } \\
\text { temperature } \\
\text { ('C) }\end{array}$ & $\begin{array}{c}\text { SC } \\
(\mu \mathbf{S} / \mathbf{c m})\end{array}$ & $\begin{array}{c}\text { DOsat } \\
\text { (percent) }\end{array}$ & $\begin{array}{c}\text { DO } \\
\text { (mg/L) }\end{array}$ & $\begin{array}{c}\mathrm{pH} \\
\text { (standard } \\
\text { units) }\end{array}$ \\
\hline $7 / 26 / 1999$ & 17:00:40 & 29.92 & 427 & 127.5 & 9.6 & 8.7 \\
\hline $7 / 26 / 1999$ & $17: 30: 40$ & 30.04 & 426 & 130.2 & 9.8 & 8.8 \\
\hline $7 / 26 / 1999$ & 18:00:40 & 30.14 & 426 & 132.4 & 10.0 & 8.8 \\
\hline $7 / 26 / 1999$ & $18: 30: 40$ & 30.12 & 425 & 132.9 & $10.0^{\mathbf{a}}$ & 8.8 \\
\hline $7 / 26 / 1999$ & 19:00:40 & 30.09 & 425 & 132.5 & 10.0 & 8.8 \\
\hline $7 / 26 / 1999$ & $19: 30: 40$ & 30.02 & 424 & 131.5 & 9.9 & 8.8 \\
\hline $7 / 26 / 1999$ & 20:00:40 & 29.9 & 424 & 129.9 & 9.8 & 8.8 \\
\hline $7 / 26 / 1999$ & $20: 30: 40$ & 29.72 & 424 & 128 & 9.7 & 8.8 \\
\hline $7 / 26 / 1999$ & 21:00:40 & 29.51 & 423 & 125.6 & 9.6 & 8.8 \\
\hline $7 / 26 / 1999$ & $21: 30: 40$ & 29.34 & 423 & 123 & 9.4 & 8.8 \\
\hline $7 / 26 / 1999$ & $22: 00: 40$ & 29.13 & 424 & 118.8 & 9.1 & 8.8 \\
\hline $7 / 26 / 1999$ & $22: 30: 40$ & 28.9 & 425 & 113.7 & 8.8 & 8.8 \\
\hline $7 / 26 / 1999$ & 23:00:40 & 28.63 & 425 & 107.8 & 8.3 & 8.8 \\
\hline $7 / 26 / 1999$ & $23: 30: 40$ & 28.37 & 426 & 102.1 & 7.9 & 8.8 \\
\hline 7/27/1999 & 0:00:40 & 28.11 & 427 & 96.2 & 7.5 & 8.8 \\
\hline 7/27/1999 & 0:30:40 & 27.88 & 428 & 90.2 & 7.1 & 8.7 \\
\hline 7/27/1999 & 1:00:40 & 27.65 & 429 & 84.1 & 6.6 & 8.7 \\
\hline $7 / 27 / 1999$ & 1:30:40 & 27.46 & 429 & 78.8 & 6.2 & 8.7 \\
\hline 7/27/1999 & 2:00:40 & 27.27 & 430 & 73.1 & 5.8 & 8.7 \\
\hline 7/27/1999 & 2:30:40 & 27.1 & 432 & 68.4 & 5.4 & 8.6 \\
\hline 7/27/1999 & 3:00:40 & 26.97 & 433 & 65.2 & 5.2 & 8.6 \\
\hline $7 / 27 / 1999$ & 3:30:40 & 26.85 & 433 & 62.7 & 5.0 & 8.6 \\
\hline 7/27/1999 & 4:00:40 & 26.77 & 434 & 61.3 & 4.9 & 8.5 \\
\hline 7/27/1999 & 4:30:40 & 26.69 & 434 & 60.5 & 4.9 & 8.5 \\
\hline 7/27/1999 & 5:00:40 & 26.61 & 434 & 60 & 4.8 & 8.5 \\
\hline 7/27/1999 & $5: 30: 40$ & 26.56 & 434 & 59.6 & 4.8 & 8.5 \\
\hline $7 / 27 / 1999$ & 6:00:40 & 26.5 & 434 & 59.4 & 4.8 & 8.5 \\
\hline 7/27/1999 & 6:30:40 & 26.45 & 434 & 59 & 4.8 & 8.5 \\
\hline 7/27/1999 & 7:00:40 & 26.43 & 434 & 59.2 & 4.8 & 8.5 \\
\hline 7/27/1999 & 7:30:40 & 26.4 & 434 & 59.2 & 4.8 & 8.5 \\
\hline $7 / 27 / 1999$ & 8:00:40 & 26.38 & 435 & 59.4 & 4.8 & 8.5 \\
\hline 7/27/1999 & 8:30:40 & 26.37 & 435 & 59.7 & 4.8 & 8.5 \\
\hline 7/27/1999 & 9:00:40 & 26.39 & 435 & 60.4 & 4.9 & 8.5 \\
\hline
\end{tabular}

$\begin{array}{lllcccl}7 / 21 / 1999 & 11: 30: 40 & 27.11 & 441 & 83.4 & 6.6 & 8.6 \\ 7 / 21 / 1999 & 12: 00: 40 & 27.5 & 439 & 89.5 & 7.1 & 8.7 \\ 7 / 21 / 1999 & 12: 30: 40 & 27.75 & 438 & 94.2 & 7.4 & 8.7 \\ 7 / 21 / 1999 & 13: 00: 40 & 27.95 & 438 & 98.4 & 7.7 & 8.7 \\ 7 / 21 / 1999 & 13: 30: 40 & 28 & 437 & 100.9 & 7.9 & 8.7 \\ 7 / 21 / 1999 & 14: 00: 40 & 28.17 & 438 & 104.8 & 8.2 & 8.7 \\ 7 / 21 / 1999 & 14: 30: 40 & 28.44 & 434 & 111.2 & 8.6 & 8.8 \\ 7 / 21 / 1999 & 15: 00: 40 & 28.55 & 434 & 113.5 & 8.8 & 8.8 \\ 7 / 21 / 1999 & 15: 30: 40 & 28.51 & 434 & 109.2 & 8.5 & 8.8 \\ 7 / 21 / 1999 & 16: 00: 40 & 28.5 & 435 & 110.3 & 8.6 & 8.8 \\ 7 / 21 / 1999 & 16: 30: 40 & 28.6 & 431 & 112.8 & 8.7 & 8.8 \\ 7 / 21 / 1999 & 17: 00: 40 & 28.31 & 431 & 106.7 & 8.3 & 8.8 \\ 7 / 21 / 1999 & 17: 30: 40 & 28.18 & 428 & 106.2 & 8.3 & 8.8 \\ 7 / 21 / 1999 & 18: 00: 40 & 28.19 & 428 & 107 & 8.3 & 8.8 \\ 7 / 21 / 1999 & 18: 30: 40 & 26.75 & 502 & 94.3 & 7.5 & 8.5\end{array}$


Appendix Continued

\begin{tabular}{|c|c|c|c|c|c|c|}
\hline Date & Time & $\begin{array}{c}\text { Water } \\
\text { temperature } \\
\left.\text { ( }{ }^{\circ} \mathrm{C}\right)\end{array}$ & $\begin{array}{c}\text { SC } \\
(\mu \mathbf{S} / \mathbf{c m})\end{array}$ & $\begin{array}{c}\text { D0sat } \\
\text { (percent) }\end{array}$ & $\begin{array}{c}\text { DO } \\
\text { (mg/L) }\end{array}$ & $\begin{array}{c}\text { pH } \\
\text { (standard } \\
\text { units) }\end{array}$ \\
\hline $7 / 21 / 1999$ & $19: 00: 40$ & 26.96 & 389 & 93.4 & 7.4 & 8.6 \\
\hline 7/21/1999 & 19:30:40 & 27.15 & 349 & 94.4 & 7.5 & 8.7 \\
\hline $7 / 21 / 1999$ & 20:00:40 & 27.33 & 373 & 94.4 & 7.5 & 8.7 \\
\hline $7 / 21 / 1999$ & $20: 30: 40$ & 27.43 & 386 & 91.8 & 7.3 & 8.7 \\
\hline $7 / 21 / 1999$ & 21:00:40 & 27.39 & 389 & 88 & 7.0 & 8.7 \\
\hline $7 / 21 / 1999$ & $21: 30: 40$ & 27.3 & 391 & 84.1 & 6.7 & 8.7 \\
\hline $7 / 21 / 1999$ & $22: 00: 40$ & 27.14 & 392 & 80.6 & 6.4 & 8.7 \\
\hline $7 / 21 / 1999$ & $22: 30: 40$ & 27.01 & 396 & 78.3 & 6.2 & 8.7 \\
\hline $7 / 21 / 1999$ & $23: 00: 40$ & 26.95 & 399 & 76 & 6.1 & 8.7 \\
\hline $7 / 21 / 1999$ & $23: 30: 40$ & 26.93 & 404 & 75.1 & 6.0 & 8.7 \\
\hline $7 / 22 / 1999$ & 0:00:40 & 26.86 & 407 & 73.8 & 5.9 & 8.7 \\
\hline $7 / 22 / 1999$ & 0:30:40 & 26.8 & 410 & 72.1 & 5.8 & 8.7 \\
\hline $7 / 22 / 1999$ & 1:00:40 & 26.73 & 412 & 70.2 & 5.6 & 8.6 \\
\hline $7 / 22 / 1999$ & $1: 30: 40$ & 26.66 & 413 & 68.3 & 5.5 & 8.6 \\
\hline $7 / 22 / 1999$ & 2:00:40 & 26.6 & 413 & 67 & 5.4 & 8.6 \\
\hline $7 / 22 / 1999$ & $2: 30: 40$ & 26.55 & 414 & 66.3 & 5.3 & 8.6 \\
\hline $7 / 22 / 1999$ & 3:00:40 & 26.51 & 415 & 65.9 & 5.3 & 8.6 \\
\hline $7 / 22 / 1999$ & $3: 30: 40$ & 26.48 & 415 & 65.5 & 5.3 & 8.6 \\
\hline $7 / 22 / 1999$ & 4:00:40 & 26.44 & 415 & 65.4 & 5.3 & 8.6 \\
\hline $7 / 22 / 1999$ & 4:30:40 & 26.41 & 416 & 65.2 & 5.3 & 8.6 \\
\hline $7 / 22 / 1999$ & 5:00:40 & 26.38 & 416 & 65.2 & 5.2 & 8.6 \\
\hline $7 / 22 / 1999$ & $5: 30: 40$ & 26.36 & 416 & 65.3 & 5.3 & 8.6 \\
\hline $7 / 22 / 1999$ & 6:00:40 & 26.32 & 420 & 65.3 & 5.3 & 8.6 \\
\hline $7 / 22 / 1999$ & $6: 30: 40$ & 26.3 & 417 & 65.8 & 5.3 & 8.6 \\
\hline $7 / 22 / 1999$ & 7:00:40 & 26.31 & 418 & 66.5 & 5.4 & 8.6 \\
\hline $7 / 22 / 1999$ & 7:30:40 & 26.29 & 428 & 67.2 & 5.4 & 8.5 \\
\hline $7 / 22 / 1999$ & 8:00:40 & 26.32 & 424 & 68.3 & 5.5 & 8.6 \\
\hline $7 / 22 / 1999$ & $8: 30: 40$ & 26.37 & 423 & 69.7 & 5.6 & 8.6 \\
\hline $7 / 22 / 1999$ & 9:00:40 & 26.46 & 423 & 72 & 5.8 & 8.6 \\
\hline $7 / 22 / 1999$ & 9:30:40 & 26.66 & 424 & 74.9 & 6.0 & 8.6 \\
\hline $7 / 22 / 1999$ & 10:00:40 & 26.95 & 422 & 77.8 & 6.2 & 8.6 \\
\hline $7 / 22 / 1999$ & $10: 30: 40$ & 27.26 & 420 & 81.2 & 6.4 & 8.6 \\
\hline $7 / 22 / 1999$ & 11:00:40 & 27.6 & 416 & 86.1 & 6.8 & 8.6 \\
\hline $7 / 22 / 1999$ & $11: 30: 40$ & 27.86 & 421 & 91.6 & 7.2 & 8.6 \\
\hline $7 / 22 / 1999$ & $12: 00: 40$ & 28.2 & 420 & 97.3 & 7.6 & 8.7 \\
\hline $7 / 22 / 1999$ & $12: 30: 40$ & 28.49 & 418 & 102.2 & 7.9 & 8.7 \\
\hline $7 / 22 / 1999$ & 13:00:40 & 28.64 & 419 & 105.8 & 8.2 & 8.7 \\
\hline $7 / 22 / 1999$ & $13: 30: 40$ & 28.7 & 417 & 108 & 8.3 & 8.7 \\
\hline $7 / 22 / 1999$ & 14:00:40 & 28.78 & 418 & 108.9 & 8.4 & 8.8 \\
\hline $7 / 22 / 1999$ & $14: 30: 40$ & 29.02 & 415 & 112.6 & 8.7 & 8.8 \\
\hline $7 / 22 / 1999$ & $15: 00: 40$ & 29.12 & 415 & 112.6 & 8.6 & 8.8 \\
\hline $7 / 22 / 1999$ & $15: 30: 40$ & 29.24 & 415 & 112.4 & 8.6 & 8.8 \\
\hline $7 / 22 / 1999$ & $16: 00: 40$ & 29.24 & 415 & 112 & 8.6 & 8.8 \\
\hline $7 / 22 / 1999$ & $16: 30: 40$ & 29.17 & 415 & 109.4 & 8.4 & 8.8 \\
\hline $7 / 22 / 1999$ & 17:00:40 & 29.14 & 415 & 107.9 & 8.3 & 8.8 \\
\hline $7 / 22 / 1999$ & $17: 30: 40$ & 29.13 & 415 & 106.8 & 8.2 & 8.8 \\
\hline $7 / 22 / 1999$ & 18:00:40 & 29.14 & 415 & 106 & 8.1 & 8.8 \\
\hline $7 / 22 / 1999$ & 18:30:40 & 29.14 & 415 & 106.6 & 8.2 & 8.8 \\
\hline $7 / 22 / 1999$ & 19:00:40 & 29.12 & 415 & 107.2 & 8.2 & 8.8 \\
\hline $7 / 22 / 1999$ & $19: 30: 40$ & 29.06 & 416 & 106.6 & 8.2 & 8.8 \\
\hline $7 / 22 / 1999$ & 20:00:40 & 28.9 & 417 & 103.6 & 8.0 & 8.8 \\
\hline
\end{tabular}


72 Water-Quality Synoptic Sampling, July 1999: North Fork Shenandoah River, Virginia

Appendix Continued

\begin{tabular}{|c|c|c|c|c|c|c|}
\hline Date & Time & $\begin{array}{c}\text { Water } \\
\text { temperature } \\
\left.\text { ( }{ }^{\circ} \mathrm{C}\right)\end{array}$ & $\begin{array}{c}\text { SC } \\
(\mu \mathbf{S} / \mathbf{c m})\end{array}$ & $\begin{array}{c}\text { DOsat } \\
\text { (percent) }\end{array}$ & $\begin{array}{c}\text { DO } \\
\text { (mg/L) }\end{array}$ & $\begin{array}{c}\mathrm{pH} \\
\text { (standard } \\
\text { units) }\end{array}$ \\
\hline $7 / 22 / 1999$ & $20: 30: 40$ & 28.71 & 418 & 101.3 & 7.8 & 8.8 \\
\hline $7 / 22 / 1999$ & 21:00:40 & 28.47 & 418 & 98.8 & 7.7 & 8.8 \\
\hline $7 / 22 / 1999$ & $21: 30: 40$ & 28.22 & 418 & 95.2 & 7.4 & 8.8 \\
\hline $7 / 22 / 1999$ & $22: 00: 40$ & 27.98 & 420 & 91.5 & 7.2 & 8.7 \\
\hline $7 / 22 / 1999$ & $22: 30: 40$ & 27.78 & 421 & 87.8 & 6.9 & 8.7 \\
\hline $7 / 22 / 1999$ & 23:00:40 & 27.6 & 422 & 83.7 & 6.6 & 8.7 \\
\hline $7 / 22 / 1999$ & $23: 30: 40$ & 27.46 & 423 & 80 & 6.3 & 8.7 \\
\hline $7 / 23 / 1999$ & 0:00:40 & 27.34 & 424 & 76.1 & 6.0 & 8.6 \\
\hline $7 / 23 / 1999$ & $0: 30: 40$ & 27.28 & 424 & 72.2 & 5.7 & 8.6 \\
\hline $7 / 23 / 1999$ & 1:00:40 & 27.29 & 424 & 70.1 & 5.6 & 8.6 \\
\hline $7 / 23 / 1999$ & $1: 30: 40$ & 27.31 & 427 & 68.4 & 5.4 & 8.6 \\
\hline $7 / 23 / 1999$ & 2:00:40 & 27.34 & 424 & 67 & 5.3 & 8.6 \\
\hline $7 / 23 / 1999$ & 2:30:40 & 27.33 & 425 & 66.4 & 5.3 & 8.6 \\
\hline $7 / 23 / 1999$ & 3:00:40 & 27.3 & 425 & 66.4 & 5.3 & 8.5 \\
\hline $7 / 23 / 1999$ & $3: 30: 40$ & 27.25 & 427 & 66.1 & 5.2 & 8.5 \\
\hline $7 / 23 / 1999$ & 4:00:40 & 27.2 & 426 & 65.9 & 5.2 & 8.5 \\
\hline $7 / 23 / 1999$ & 4:30:40 & 27.14 & 427 & 66 & 5.2 & 8.5 \\
\hline $7 / 23 / 1999$ & 5:00:40 & 27.07 & 427 & 65.9 & 5.2 & 8.5 \\
\hline $7 / 23 / 1999$ & $5: 30: 40$ & 26.99 & 428 & 66.1 & 5.3 & 8.5 \\
\hline $7 / 23 / 1999$ & 6:00:40 & 26.93 & 428 & 65.9 & 5.3 & 8.5 \\
\hline $7 / 23 / 1999$ & $6: 30: 40$ & 26.87 & 428 & 66 & 5.3 & 8.5 \\
\hline $7 / 23 / 1999$ & 7:00:40 & 26.82 & 429 & 67 & 5.4 & 8.5 \\
\hline $7 / 23 / 1999$ & 7:30:40 & 26.8 & 430 & 68 & 5.4 & 8.5 \\
\hline $7 / 23 / 1999$ & 8:00:40 & 26.8 & 432 & 69.1 & 5.5 & 8.5 \\
\hline $7 / 23 / 1999$ & $8: 30: 40$ & 26.87 & 432 & 70.7 & 5.6 & 8.5 \\
\hline $7 / 23 / 1999$ & 9:00:40 & 26.98 & 436 & 73 & 5.8 & 8.5 \\
\hline $7 / 23 / 1999$ & $9: 30: 40$ & 27.2 & 435 & 75.9 & 6.0 & 8.5 \\
\hline 7/23/1999 & 10:00:40 & 27.48 & 435 & 79.9 & 6.3 & 8.5 \\
\hline $7 / 23 / 1999$ & $10: 30: 40$ & 27.74 & 435 & 83.3 & 6.5 & 8.5 \\
\hline $7 / 23 / 1999$ & 11:00:40 & 28.01 & 435 & 86.8 & 6.8 & 8.5 \\
\hline $7 / 23 / 1999$ & 11:30:40 & 28.33 & 435 & 91 & 7.1 & 8.6 \\
\hline $7 / 23 / 1999$ & $12: 00: 40$ & 28.62 & 436 & 95 & 7.4 & 8.6 \\
\hline $7 / 23 / 1999$ & $12: 30: 40$ & 28.99 & 434 & 99.8 & 7.7 & 8.6 \\
\hline $7 / 23 / 1999$ & 13:00:40 & 29.32 & 434 & 104.4 & 8.0 & 8.6 \\
\hline $7 / 23 / 1999$ & $13: 30: 40$ & 29.59 & 433 & 107.8 & 8.2 & 8.6 \\
\hline $7 / 23 / 1999$ & 14:00:40 & 29.87 & 434 & 112.1 & 8.5 & 8.7 \\
\hline $7 / 23 / 1999$ & $14: 30: 40$ & 30.04 & 434 & 114.8 & 8.7 & 8.7 \\
\hline $7 / 23 / 1999$ & $15: 00: 40$ & 30.26 & 432 & 117.8 & 8.9 & 8.7 \\
\hline $7 / 23 / 1999$ & $15: 30: 40$ & 30.46 & 429 & 120.4 & 9.0 & 8.7 \\
\hline $7 / 23 / 1999$ & $16: 00: 40$ & 30.57 & 431 & 122.4 & 9.2 & 8.7 \\
\hline $7 / 23 / 1999$ & $16: 30: 40$ & 30.78 & 428 & 124.6 & 9.3 & 8.7 \\
\hline $7 / 23 / 1999$ & 17:00:40 & 30.8 & 428 & 123.8 & 9.2 & 8.7 \\
\hline $7 / 23 / 1999$ & $17: 30: 40$ & 30.91 & 426 & 124.7 & 9.3 & 8.8 \\
\hline $7 / 23 / 1999$ & 18:00:40 & 30.83 & 425 & 123.4 & 9.2 & 8.8 \\
\hline $7 / 23 / 1999$ & $18: 30: 40$ & 30.76 & 423 & 121.6 & 9.1 & 8.8 \\
\hline $7 / 23 / 1999$ & 19:00:40 & 30.65 & 422 & 119.2 & 8.9 & 8.7 \\
\hline $7 / 23 / 1999$ & $19: 30: 40$ & 30.54 & 422 & 116.7 & 8.7 & 8.7 \\
\hline $7 / 23 / 1999$ & 20:00:40 & 30.39 & 422 & 113.8 & 8.5 & 8.7 \\
\hline 7/23/1999 & $20: 30: 40$ & 30.22 & 422 & 110.3 & 8.3 & 8.7 \\
\hline $7 / 23 / 1999$ & 21:00:40 & 30 & 420 & 107.8 & 8.1 & 8.7 \\
\hline
\end{tabular}


Appendix Continued

\begin{tabular}{|c|c|c|c|c|c|c|}
\hline Date & Time & $\begin{array}{c}\text { Water } \\
\text { temperature } \\
\left.\text { ( }{ }^{\circ} \mathrm{C}\right)\end{array}$ & $\begin{array}{c}\text { SC } \\
(\mu \mathrm{S} / \mathrm{cm})\end{array}$ & $\begin{array}{c}\text { DOsat } \\
\text { (percent) }\end{array}$ & $\begin{array}{c}\text { DO } \\
\text { (mg/L) }\end{array}$ & $\begin{array}{c}\mathrm{pH} \\
\text { (standard } \\
\text { units) }\end{array}$ \\
\hline $7 / 23 / 1999$ & $21: 30: 40$ & 29.77 & 420 & 104 & 7.9 & 8.7 \\
\hline $7 / 23 / 1999$ & 22:00:40 & 29.55 & 420 & 100.4 & 7.6 & 8.7 \\
\hline 7/23/1999 & $22: 30: 40$ & 29.36 & 422 & 96 & 7.3 & 8.7 \\
\hline $7 / 23 / 1999$ & 23:00:40 & 29.18 & 424 & 91.4 & 7.0 & 8.7 \\
\hline $7 / 23 / 1999$ & $23: 30: 40$ & 28.99 & 426 & 85.7 & 6.6 & 8.6 \\
\hline $7 / 24 / 1999$ & 0:00:40 & 28.83 & 427 & 81.2 & 6.3 & 8.6 \\
\hline 7/24/1999 & $0: 30: 40$ & 28.66 & 428 & 78 & 6.0 & 8.6 \\
\hline $7 / 24 / 1999$ & 1:00:40 & 28.5 & 428 & 74.2 & 5.8 & 8.6 \\
\hline 7/24/1999 & $1: 30: 40$ & 28.33 & 429 & 70.8 & 5.5 & 8.5 \\
\hline $7 / 24 / 1999$ & 2:00:40 & 28.18 & 429 & 67.9 & 5.3 & 8.5 \\
\hline $7 / 24 / 1999$ & $2: 30: 40$ & 28.01 & 430 & 65.7 & 5.1 & 8.5 \\
\hline $7 / 24 / 1999$ & 3:00:40 & 27.85 & 431 & 64.1 & 5.0 & 8.5 \\
\hline $7 / 24 / 1999$ & 3:30:40 & 27.69 & 430 & 62.9 & 5.0 & 8.4 \\
\hline $7 / 24 / 1999$ & 4:00:40 & 27.51 & 431 & 62.4 & $4.9^{\mathbf{b}}$ & 8.4 \\
\hline $7 / 24 / 1999$ & $4: 30: 40$ & 27.34 & 431 & 62.4 & 4.9 & 8.4 \\
\hline 7/24/1999 & 5:00:40 & 27.2 & 432 & 62.3 & 4.9 & 8.4 \\
\hline $7 / 24 / 1999$ & $5: 30: 40$ & 27.09 & 432 & 62.3 & 5.0 & 8.4 \\
\hline 7/24/1999 & 6:00:40 & 27 & 432 & 62.2 & 5.0 & 8.4 \\
\hline $7 / 24 / 1999$ & $6: 30: 40$ & 26.92 & 432 & 62.3 & 5.0 & 8.4 \\
\hline 7/24/1999 & 7:00:40 & 26.88 & 431 & 63.3 & 5.1 & 8.4 \\
\hline $7 / 24 / 1999$ & $7: 30: 40$ & 26.84 & 432 & 63.6 & 5.1 & 8.3 \\
\hline 7/24/1999 & 8:00:40 & 26.85 & 434 & 65.8 & 5.3 & 8.4 \\
\hline $7 / 24 / 1999$ & $8: 30: 40$ & 26.88 & 434 & 68.1 & 5.4 & 8.4 \\
\hline $7 / 24 / 1999$ & 9:00:40 & 27.04 & 436 & 71.8 & 5.7 & 8.4 \\
\hline $7 / 24 / 1999$ & $9: 30: 40$ & 27.15 & 437 & 73.7 & 5.9 & 8.4 \\
\hline $7 / 24 / 1999$ & 10:00:40 & 27.36 & 437 & 77.1 & 6.1 & 8.4 \\
\hline $7 / 24 / 1999$ & $10: 30: 40$ & 27.5 & 438 & 79.2 & 6.3 & 8.4 \\
\hline $7 / 24 / 1999$ & 11:00:40 & 27.72 & 438 & 81.7 & 6.4 & 8.4 \\
\hline $7 / 24 / 1999$ & $11: 30: 40$ & 27.74 & 440 & 80.3 & 6.3 & 8.4 \\
\hline $7 / 24 / 1999$ & $12: 00: 40$ & 27.79 & 439 & 83.8 & 6.6 & 8.4 \\
\hline $7 / 24 / 1999$ & $12: 30: 40$ & 28.03 & 437 & 91.4 & 7.1 & 8.5 \\
\hline $7 / 24 / 1999$ & 13:00:40 & 28.03 & 436 & 91.5 & 7.2 & 8.5 \\
\hline $7 / 24 / 1999$ & $13: 30: 40$ & 28.31 & 438 & 98.9 & 7.7 & 8.5 \\
\hline 7/24/1999 & $14: 00: 40$ & 28.79 & 433 & 106.4 & 8.2 & 8.6 \\
\hline $7 / 24 / 1999$ & $14: 30: 40$ & 29.11 & 433 & 110.9 & 8.5 & 8.6 \\
\hline 7/24/1999 & $15: 00: 40$ & 29.27 & 433 & 113.7 & 8.7 & 8.6 \\
\hline $7 / 24 / 1999$ & $15: 30: 40$ & 29.39 & 432 & 115 & 8.8 & 8.7 \\
\hline 7/24/1999 & 16:00:40 & 29.41 & 431 & 113.7 & 8.7 & 8.7 \\
\hline 7/24/1999 & $16: 30: 40$ & 29.49 & 430 & 113.2 & 8.6 & 8.7 \\
\hline $7 / 24 / 1999$ & 17:00:40 & 29.5 & 430 & 112.7 & 8.6 & 8.7 \\
\hline $7 / 24 / 1999$ & $17: 30: 40$ & 29.5 & 431 & 114.1 & 8.7 & 8.7 \\
\hline $7 / 24 / 1999$ & 18:00:40 & 29.5 & 431 & 112.9 & 8.6 & 8.7 \\
\hline 7/24/1999 & $18: 30: 40$ & 29.54 & 431 & 114.1 & 8.7 & 8.7 \\
\hline $7 / 24 / 1999$ & 19:00:40 & 29.54 & 430 & 116.1 & 8.8 & 8.7 \\
\hline $7 / 24 / 1999$ & 19:30:40 & 29.42 & 432 & 112.4 & 8.6 & 8.7 \\
\hline 7/24/1999 & 20:00:40 & 29.33 & 431 & 110 & 8.4 & 8.7 \\
\hline $7 / 24 / 1999$ & $20: 30: 40$ & 29.21 & 431 & 106.1 & 8.1 & 8.7 \\
\hline 7/24/1999 & 21:00:40 & 29.09 & 430 & 103.1 & 7.9 & 8.7 \\
\hline $7 / 24 / 1999$ & $21: 30: 40$ & 28.97 & 431 & 100.3 & 7.7 & 8.7 \\
\hline 7/24/1999 & 22:00:40 & 28.8 & 433 & 96.1 & 7.4 & 8.7 \\
\hline
\end{tabular}


Water-Quality Synoptic Sampling, July 1999: North Fork Shenandoah River, Virginia

Appendix Continued

\begin{tabular}{|c|c|c|c|c|c|c|}
\hline Date & Time & $\begin{array}{c}\text { Water } \\
\text { temperature } \\
\text { ('C) }\end{array}$ & $\begin{array}{c}\text { SC } \\
(\mu \mathrm{S} / \mathbf{c m})\end{array}$ & $\begin{array}{c}\text { D0sat } \\
\text { (percent) }\end{array}$ & $\begin{array}{c}\text { DO } \\
\text { (mg/L) }\end{array}$ & $\begin{array}{c}\text { pH } \\
\text { (standard } \\
\text { units) }\end{array}$ \\
\hline $7 / 24 / 1999$ & $22: 30: 40$ & 28.59 & 433 & 91.5 & 7.1 & 8.6 \\
\hline $7 / 24 / 1999$ & 23:00:40 & 28.35 & 434 & 86.6 & 6.7 & 8.6 \\
\hline $7 / 24 / 1999$ & $23: 30: 40$ & 28.13 & 433 & 82 & 6.4 & 8.6 \\
\hline $7 / 25 / 1999$ & 0:00:40 & 27.94 & 433 & 78.1 & 6.1 & 8.5 \\
\hline $7 / 25 / 1999$ & 0:30:40 & 27.77 & 434 & 74.9 & 5.9 & 8.5 \\
\hline $7 / 25 / 1999$ & 1:00:40 & 27.63 & 435 & 72.3 & 5.7 & 8.5 \\
\hline $7 / 25 / 1999$ & 1:30:40 & 27.54 & 433 & 70.1 & 5.5 & 8.5 \\
\hline $7 / 25 / 1999$ & 2:00:40 & 27.43 & 433 & 67.9 & 5.4 & 8.5 \\
\hline $7 / 25 / 1999$ & 2:30:40 & 27.35 & 432 & 66 & 5.2 & 8.5 \\
\hline 7/25/1999 & 3:00:40 & 27.27 & 433 & 64.9 & 5.1 & 8.4 \\
\hline $7 / 25 / 1999$ & $3: 30: 40$ & 27.21 & 432 & 63.9 & 5.1 & 8.4 \\
\hline $7 / 25 / 1999$ & 4:00:40 & 27.13 & 432 & 63.4 & 5.0 & 8.4 \\
\hline $7 / 25 / 1999$ & $4: 30: 40$ & 27.08 & 430 & 63 & 5.0 & 8.4 \\
\hline $7 / 25 / 1999$ & 5:00:40 & 27.02 & 431 & 62.8 & 5.0 & 8.4 \\
\hline $7 / 25 / 1999$ & $5: 30: 40$ & 26.95 & 432 & 62.8 & 5.0 & 8.4 \\
\hline $7 / 25 / 1999$ & 6:00:40 & 26.89 & 432 & 63 & 5.0 & 8.4 \\
\hline $7 / 25 / 1999$ & $6: 30: 40$ & 26.84 & 431 & 63.4 & 5.1 & 8.4 \\
\hline $7 / 25 / 1999$ & 7:00:40 & 26.79 & 432 & 64.4 & 5.2 & 8.4 \\
\hline $7 / 25 / 1999$ & 7:30:40 & 26.75 & 432 & 65.3 & 5.2 & 8.4 \\
\hline $7 / 25 / 1999$ & 8:00:40 & 26.75 & 432 & 66.6 & 5.3 & 8.4 \\
\hline $7 / 25 / 1999$ & 8:30:40 & 26.82 & 435 & 68.9 & 5.5 & 8.4 \\
\hline $7 / 25 / 1999$ & 9:00:40 & 26.93 & 437 & 71.6 & 5.7 & 8.4 \\
\hline $7 / 25 / 1999$ & $9: 30: 40$ & 27.12 & 437 & 74.9 & 6.0 & 8.4 \\
\hline $7 / 25 / 1999$ & 10:00:40 & 27.42 & 439 & 79.5 & 6.3 & 8.4 \\
\hline $7 / 25 / 1999$ & $10: 30: 40$ & 27.65 & 440 & 82.5 & 6.5 & 8.4 \\
\hline $7 / 25 / 1999$ & 11:00:40 & 27.86 & 438 & 85.4 & 6.7 & 8.5 \\
\hline $7 / 25 / 1999$ & 11:30:40 & 28.18 & 440 & 89.5 & 7.0 & 8.5 \\
\hline $7 / 25 / 1999$ & $12: 00: 40$ & 28.31 & 439 & 90.9 & 7.1 & 8.5 \\
\hline $7 / 25 / 1999$ & $12: 30: 40$ & 28.45 & 439 & 93.8 & 7.3 & 8.5 \\
\hline $7 / 25 / 1999$ & 13:00:40 & 28.7 & 438 & 98.1 & 7.6 & 8.5 \\
\hline $7 / 25 / 1999$ & $13: 30: 40$ & 28.97 & 436 & 103 & 7.9 & 8.6 \\
\hline $7 / 25 / 1999$ & 14:00:40 & 29.29 & 434 & 109.2 & 8.4 & 8.6 \\
\hline $7 / 25 / 1999$ & $14: 30: 40$ & 29.53 & 438 & 113.9 & 8.7 & 8.6 \\
\hline $7 / 25 / 1999$ & $15: 00: 40$ & 29.57 & 434 & 115.7 & 8.8 & 8.6 \\
\hline $7 / 25 / 1999$ & $15: 30: 40$ & 29.71 & 433 & 118.1 & 9.0 & 8.7 \\
\hline $7 / 25 / 1999$ & 16:00:40 & 29.81 & 433 & 119.5 & 9.1 & 8.7 \\
\hline $7 / 25 / 1999$ & $16: 30: 40$ & 29.86 & 431 & 120.6 & 9.1 & 8.7 \\
\hline $7 / 25 / 1999$ & 17:00:40 & 29.89 & 429 & 121.6 & 9.2 & 8.7 \\
\hline $7 / 25 / 1999$ & $17: 30: 40$ & 29.81 & 429 & 121.1 & 9.2 & 8.7 \\
\hline $7 / 25 / 1999$ & 18:00:40 & 29.77 & 424 & 120.4 & 9.1 & 8.7 \\
\hline $7 / 25 / 1999$ & $18: 30: 40$ & 29.73 & 431 & 119.5 & 9.1 & 8.7 \\
\hline $7 / 25 / 1999$ & 19:00:40 & 29.73 & 431 & 118.4 & 9.0 & 8.7 \\
\hline $7 / 25 / 1999$ & $19: 30: 40$ & 29.71 & 430 & 117 & 8.9 & 8.7 \\
\hline $7 / 25 / 1999$ & 20:00:40 & 29.72 & 431 & 116.3 & 8.8 & 8.7 \\
\hline $7 / 25 / 1999$ & $20: 30: 40$ & 29.68 & 431 & 115.5 & 8.8 & 8.7 \\
\hline $7 / 25 / 1999$ & 21:00:40 & 29.57 & 434 & 114 & 8.7 & 8.7 \\
\hline $7 / 25 / 1999$ & $21: 30: 40$ & 29.43 & 431 & 111.5 & 8.5 & 8.7 \\
\hline $7 / 25 / 1999$ & 22:00:40 & 29.26 & 430 & 107.8 & 8.2 & 8.7 \\
\hline $7 / 25 / 1999$ & $22: 30: 40$ & 29.02 & 432 & 103.2 & 7.9 & 8.7 \\
\hline $7 / 25 / 1999$ & 23:00:40 & 28.83 & 431 & 99.1 & 7.6 & 8.7 \\
\hline
\end{tabular}


Appendix Continued

\begin{tabular}{|c|c|c|c|c|c|c|}
\hline Date & Time & $\begin{array}{c}\text { Water } \\
\text { temperature } \\
\text { ('C) }\end{array}$ & $\begin{array}{c}\text { SC } \\
(\mu \mathrm{S} / \mathrm{cm})\end{array}$ & $\begin{array}{c}\text { DOsat } \\
\text { (percent) }\end{array}$ & $\begin{array}{c}\text { DO } \\
\text { (mg/L) }\end{array}$ & $\begin{array}{c}\text { pH } \\
\text { (standard } \\
\text { units) }\end{array}$ \\
\hline $7 / 25 / 1999$ & $23: 30: 40$ & 28.57 & 434 & 94.4 & 7.3 & 8.7 \\
\hline $7 / 26 / 1999$ & 0:00:40 & 28.41 & 431 & 89.3 & 6.9 & 8.6 \\
\hline $7 / 26 / 1999$ & $0: 30: 40$ & 28.21 & 433 & 85.1 & 6.6 & 8.6 \\
\hline $7 / 26 / 1999$ & 1:00:40 & 28.05 & 432 & 80.7 & 6.3 & 8.6 \\
\hline $7 / 26 / 1999$ & $1: 30: 40$ & 27.9 & 431 & 76 & 6.0 & 8.6 \\
\hline $7 / 26 / 1999$ & 2:00:40 & 27.75 & 432 & 72.4 & 5.7 & 8.5 \\
\hline $7 / 26 / 1999$ & $2: 30: 40$ & 27.62 & 433 & 68.5 & 5.4 & 8.5 \\
\hline $7 / 26 / 1999$ & 3:00:40 & 27.49 & 433 & 66.1 & 5.2 & 8.5 \\
\hline $7 / 26 / 1999$ & 3:30:40 & 27.37 & 434 & 64.3 & 5.1 & 8.4 \\
\hline $7 / 26 / 1999$ & 4:00:40 & 27.27 & 433 & 63 & 5.0 & 8.4 \\
\hline $7 / 26 / 1999$ & 4:30:40 & 27.15 & 434 & 62.5 & 5.0 & 8.4 \\
\hline $7 / 26 / 1999$ & 5:00:40 & 27.02 & 434 & 62.3 & 5.0 & 8.4 \\
\hline $7 / 26 / 1999$ & $5: 30: 40$ & 26.92 & 435 & 62.3 & 5.0 & 8.4 \\
\hline $7 / 26 / 1999$ & 6:00:40 & 26.81 & 435 & 62.4 & 5.0 & 8.4 \\
\hline $7 / 26 / 1999$ & $6: 30: 40$ & 26.73 & 436 & 62.1 & 5.0 & 8.4 \\
\hline $7 / 26 / 1999$ & 7:00:40 & 26.65 & 436 & 62.9 & 5.0 & 8.4 \\
\hline $7 / 26 / 1999$ & 7:30:40 & 26.58 & 437 & 64.4 & 5.2 & 8.4 \\
\hline $7 / 26 / 1999$ & 8:00:40 & 26.55 & 439 & 66.2 & 5.3 & 8.4 \\
\hline $7 / 26 / 1999$ & $8: 30: 40$ & 26.54 & 440 & 67.7 & 5.4 & 8.4 \\
\hline $7 / 26 / 1999$ & 9:00:40 & 26.56 & 442 & 68.5 & 5.5 & 8.4 \\
\hline $7 / 26 / 1999$ & $9: 30: 40$ & 26.65 & 440 & 70.7 & 5.7 & 8.4 \\
\hline $7 / 26 / 1999$ & 10:00:40 & 26.67 & 442 & 71.3 & 5.7 & 8.4 \\
\hline $7 / 26 / 1999$ & $10: 30: 40$ & 26.74 & 442 & 73.7 & 5.9 & 8.4 \\
\hline $7 / 26 / 1999$ & 11:00:40 & 27.04 & 443 & 79.1 & 6.3 & 8.4 \\
\hline $7 / 26 / 1999$ & $11: 30: 40$ & 27.33 & 441 & 82.7 & 6.5 & 8.5 \\
\hline $7 / 26 / 1999$ & $12: 00: 40$ & 27.69 & 440 & 87.5 & 6.9 & 8.5 \\
\hline $7 / 26 / 1999$ & $12: 30: 40$ & 27.87 & 439 & 90.6 & 7.1 & 8.5 \\
\hline $7 / 26 / 1999$ & 13:00:40 & 28.09 & 441 & 94.6 & 7.4 & 8.5 \\
\hline $7 / 26 / 1999$ & $13: 30: 40$ & 28.31 & 440 & 98.1 & 7.6 & 8.5 \\
\hline $7 / 26 / 1999$ & 14:00:40 & 28.29 & 441 & 100.7 & 7.8 & 8.5 \\
\hline $7 / 26 / 1999$ & $14: 30: 40$ & 28.57 & 440 & 105.9 & 8.2 & 8.6 \\
\hline $7 / 26 / 1999$ & $15: 00: 40$ & 28.69 & 438 & 108.8 & 8.4 & 8.6 \\
\hline $7 / 26 / 1999$ & $15: 30: 40$ & 28.95 & 438 & 113.6 & 8.7 & 8.6 \\
\hline $7 / 26 / 1999$ & 16:00:40 & 29.05 & 438 & 115.3 & 8.9 & 8.6 \\
\hline $7 / 26 / 1999$ & $16: 30: 40$ & 29.32 & 433 & 119.6 & 9.1 & 8.7 \\
\hline $7 / 26 / 1999$ & 17:00:40 & 29.4 & 436 & 121.1 & 9.2 & 8.7 \\
\hline $7 / 26 / 1999$ & $17: 30: 40$ & 29.57 & 434 & 122.8 & 9.3 & 8.7 \\
\hline $7 / 26 / 1999$ & 18:00:40 & 29.66 & 433 & 124 & $9.4^{\mathrm{a}}$ & 8.7 \\
\hline $7 / 26 / 1999$ & 18:30:40 & 29.68 & 432 & 123.4 & 9.4 & 8.8 \\
\hline $7 / 26 / 1999$ & 19:00:40 & 29.64 & 432 & 122.9 & 9.3 & 8.8 \\
\hline $7 / 26 / 1999$ & $19: 30: 40$ & 29.62 & 431 & 121.4 & 9.2 & 8.8 \\
\hline $7 / 26 / 1999$ & 20:00:40 & 29.56 & 431 & 119.2 & 9.1 & 8.8 \\
\hline $7 / 26 / 1999$ & $20: 30: 40$ & 29.39 & 433 & 116.6 & 8.9 & 8.8 \\
\hline $7 / 26 / 1999$ & 21:00:40 & 29.22 & 432 & 114.2 & 8.7 & 8.8 \\
\hline $7 / 26 / 1999$ & $21: 30: 40$ & 29.04 & 431 & 112.6 & 8.6 & 8.8 \\
\hline $7 / 26 / 1999$ & $22: 00: 40$ & 28.86 & 432 & 109.9 & 8.5 & 8.8 \\
\hline $7 / 26 / 1999$ & $22: 30: 40$ & 28.66 & 431 & 106.4 & 8.2 & 8.7 \\
\hline $7 / 26 / 1999$ & 23:00:40 & 28.47 & 429 & 102.3 & 7.9 & 8.7 \\
\hline $7 / 26 / 1999$ & $23: 30: 40$ & 28.21 & 433 & 98.1 & 7.6 & 8.7 \\
\hline 7/27/1999 & 0:00:40 & 27.97 & 433 & 93.7 & 7.3 & 8.7 \\
\hline $7 / 27 / 1999$ & $0: 30: 40$ & 27.74 & 432 & 89.4 & 7.0 & 8.7 \\
\hline
\end{tabular}


Water-Quality Synoptic Sampling, July 1999: North Fork Shenandoah River, Virginia

Appendix Continued

\begin{tabular}{ccccccc}
\hline Date & Time & $\begin{array}{c}\text { Water } \\
\text { temperature } \\
(\mathbf{0} \text { ) }\end{array}$ & $\begin{array}{c}\text { SC } \\
(\mu \mathbf{S} / \mathbf{c m})\end{array}$ & $\begin{array}{c}\text { DOsat } \\
\text { (percent) }\end{array}$ & $\begin{array}{c}\text { DO } \\
(\mathbf{m g} / \mathbf{L})\end{array}$ & $\begin{array}{c}\text { pH } \\
\text { (standard } \\
\text { units) }\end{array}$ \\
\hline $7 / 27 / 1999$ & $1: 00: 40$ & 27.53 & 431 & 85 & 6.7 & 8.7 \\
$7 / 27 / 1999$ & $1: 30: 40$ & 27.33 & 431 & 80.8 & 6.4 & 8.6 \\
$7 / 27 / 1999$ & $2: 00: 40$ & 27.16 & 430 & 76.6 & 6.1 & 8.6 \\
$7 / 27 / 1999$ & $2: 30: 40$ & 26.99 & 430 & 72.4 & 5.8 & 8.6 \\
$7 / 27 / 1999$ & $3: 00: 40$ & 26.84 & 430 & 69.2 & 5.5 & 8.6 \\
$7 / 27 / 1999$ & $3: 30: 40$ & 26.69 & 430 & 66.6 & 5.3 & 8.5 \\
$7 / 27 / 1999$ & $4: 00: 40$ & 26.57 & 430 & 64.6 & 5.2 & 8.5 \\
$7 / 27 / 1999$ & $4: 30: 40$ & 26.46 & 430 & 63.6 & 5.1 & 8.5 \\
$7 / 27 / 1999$ & $5: 00: 40$ & 26.37 & 430 & 62.9 & 5.1 & 8.5 \\
$7 / 27 / 1999$ & $5: 30: 40$ & 26.31 & 430 & 62.4 & 5.0 & 8.5 \\
$7 / 27 / 1999$ & $6: 00: 40$ & 26.24 & 432 & 62.2 & 5.0 & 8.5 \\
$7 / 27 / 1999$ & $6: 30: 40$ & 26.2 & 431 & 62.6 & 5.1 & 8.5 \\
$7 / 27 / 1999$ & $7: 00: 40$ & 26.17 & 432 & 62.6 & 5.1 & 8.4 \\
$7 / 27 / 1999$ & $7: 30: 40$ & 26.14 & 434 & 62.6 & 5.1 & 8.4 \\
$7 / 27 / 1999$ & $8: 00: 40$ & 26.12 & 438 & 64 & 5.2 & 8.4 \\
$7 / 27 / 1999$ & $8: 30: 40$ & 26.12 & 439 & 65.3 & 5.3 & 8.4 \\
$7 / 27 / 1999$ & $9: 00: 40$ & 26.13 & 440 & 66.8 & 5.4 & 8.4 \\
$7 / 27 / 1999$ & $9: 30: 40$ & 26.19 & 440 & 69.2 & 5.6 & 8.4 \\
\hline
\end{tabular}

\begin{tabular}{|c|c|c|c|c|c|c|}
\hline & & & ite 48 & & & \\
\hline 7/27/1999 & $15: 00: 40$ & 28.55 & 422 & 99.5 & 7.7 & 9.1 \\
\hline 7/27/1999 & $15: 30: 40$ & 28.52 & 424 & 100.7 & 7.8 & 9.1 \\
\hline $7 / 27 / 1999$ & $16: 00: 40$ & 28.48 & 426 & 101.4 & 7.9 & 9.1 \\
\hline $7 / 27 / 1999$ & $16: 30: 40$ & 28.46 & 428 & 103.8 & 8.1 & 9.1 \\
\hline 7/27/1999 & 17:00:40 & 28.41 & 429 & 104.9 & 8.1 & 9.0 \\
\hline 7/27/1999 & $17: 30: 40$ & 28.37 & 431 & 103 & 8.0 & 9.0 \\
\hline $7 / 27 / 1999$ & 18:00:40 & 28.37 & 433 & 103 & 8.0 & 9.0 \\
\hline $7 / 27 / 1999$ & $18: 30: 40$ & 28.4 & 433 & 104.7 & 8.1 & 9.0 \\
\hline 7/27/1999 & 19:00:40 & 28.45 & 433 & 105.6 & 8.2 & 9.0 \\
\hline 7/27/1999 & $19: 30: 40$ & 28.5 & 433 & 106.8 & 8.3 & 9.0 \\
\hline 7/27/1999 & 20:00:40 & 28.53 & 432 & 106.7 & 8.3 & 9.0 \\
\hline $7 / 27 / 1999$ & $20: 30: 40$ & 28.54 & 432 & 105.6 & 8.2 & 9.0 \\
\hline 7/27/1999 & 21:00:40 & 28.51 & 432 & 104.7 & 8.1 & 9.0 \\
\hline $7 / 27 / 1999$ & $21: 30: 40$ & 28.46 & 431 & 104.3 & 8.1 & 9.0 \\
\hline $7 / 27 / 1999$ & 22:00:40 & 28.42 & 430 & 104.6 & 8.1 & 9.0 \\
\hline 7/27/1999 & $22: 30: 40$ & 28.37 & 429 & 104.7 & 8.1 & 9.1 \\
\hline 7/27/1999 & 23:00:40 & 28.29 & 428 & 104.2 & 8.1 & 9.1 \\
\hline 7/27/1999 & $23: 30: 40$ & 28.26 & 426 & 104.9 & 8.2 & 9.1 \\
\hline $7 / 28 / 1999$ & 0:00:40 & 28.19 & 424 & 104.5 & 8.2 & 9.1 \\
\hline 7/28/1999 & 0:30:40 & 28.13 & 422 & 104.4 & 8.2 & 9.1 \\
\hline $7 / 28 / 1999$ & 1:00:40 & 28.05 & 421 & 103.5 & 8.1 & 9.2 \\
\hline $7 / 28 / 1999$ & 1:30:40 & 27.97 & 420 & 102.4 & 8.0 & 9.2 \\
\hline $7 / 28 / 1999$ & 2:00:40 & 27.89 & 419 & 100.9 & 7.9 & 9.2 \\
\hline $7 / 28 / 1999$ & 2:30:40 & 27.8 & 418 & 99.3 & 7.8 & 9.2 \\
\hline $7 / 28 / 1999$ & 3:00:40 & 27.71 & 417 & 96.9 & 7.6 & 9.2 \\
\hline $7 / 28 / 1999$ & $3: 30: 40$ & 27.61 & 416 & 94.5 & 7.4 & 9.2 \\
\hline $7 / 28 / 1999$ & 4:00:40 & 27.51 & 415 & 92 & 7.3 & 9.2 \\
\hline $7 / 28 / 1999$ & 4:30:40 & 27.42 & 415 & 89.6 & 7.1 & 9.2 \\
\hline $7 / 28 / 1999$ & 5:00:40 & 27.3 & 414 & 86.8 & 6.9 & 9.3 \\
\hline $7 / 28 / 1999$ & $5: 30: 40$ & 27.19 & 413 & 84.1 & 6.7 & 9.3 \\
\hline
\end{tabular}


Appendix Continued

\begin{tabular}{|c|c|c|c|c|c|c|}
\hline Date & Time & $\begin{array}{c}\text { Water } \\
\text { temperature } \\
\text { ('C) }\end{array}$ & $\begin{array}{c}\text { SC } \\
(\mu \mathrm{S} / \mathrm{cm})\end{array}$ & $\begin{array}{c}\text { DOsat } \\
\text { (percent) }\end{array}$ & $\begin{array}{c}\text { DO } \\
\text { (mg/L) }\end{array}$ & $\begin{array}{c}\text { pH } \\
\text { (standard } \\
\text { units) }\end{array}$ \\
\hline $7 / 28 / 1999$ & $6: 00: 40$ & 27.06 & 413 & 81.2 & 6.5 & 9.3 \\
\hline $7 / 28 / 1999$ & $6: 30: 40$ & 26.94 & 413 & 78.9 & 6.3 & 9.3 \\
\hline $7 / 28 / 1999$ & 7:00:40 & 26.83 & 412 & 77.1 & 6.2 & 9.3 \\
\hline $7 / 28 / 1999$ & $7: 30: 40$ & 26.74 & 412 & 76.3 & 6.1 & 9.3 \\
\hline $7 / 28 / 1999$ & 8:00:40 & 26.7 & 412 & 76.6 & 6.1 & 9.3 \\
\hline $7 / 28 / 1999$ & $8: 30: 40$ & 26.74 & 411 & 78.5 & 6.3 & 9.3 \\
\hline $7 / 28 / 1999$ & 9:00:40 & 26.83 & 411 & 81.3 & 6.5 & 9.3 \\
\hline $7 / 28 / 1999$ & 9:30:40 & 26.96 & 410 & 84.5 & 6.7 & 9.3 \\
\hline $7 / 28 / 1999$ & 10:00:40 & 27.15 & 409 & 88 & 7.0 & 9.3 \\
\hline $7 / 28 / 1999$ & 10:30:40 & 27.43 & 409 & 92.9 & 7.3 & 9.3 \\
\hline $7 / 28 / 1999$ & 11:00:40 & 27.75 & 409 & 98.2 & 7.7 & 9.3 \\
\hline $7 / 28 / 1999$ & 11:30:40 & 28.11 & 408 & 103.1 & 8.0 & 9.3 \\
\hline 7/28/1999 & $12: 00: 40$ & 28.44 & 409 & 107.1 & 8.3 & 9.3 \\
\hline $7 / 28 / 1999$ & $12: 30: 40$ & 28.76 & 409 & 110.3 & 8.5 & 9.3 \\
\hline $7 / 28 / 1999$ & $13: 00: 40$ & 28.93 & 410 & 111.4 & $8.6^{\mathbf{a}}$ & 9.3 \\
\hline $7 / 28 / 1999$ & $13: 30: 40$ & 28.96 & 411 & 108.8 & 8.4 & 9.2 \\
\hline $7 / 28 / 1999$ & 14:00:40 & 28.83 & 413 & 105.5 & 8.1 & 9.2 \\
\hline $7 / 28 / 1999$ & $14: 30: 40$ & 28.62 & 415 & 102.1 & 7.9 & 9.2 \\
\hline $7 / 28 / 1999$ & $15: 00: 40$ & 28.38 & 417 & 96.1 & 7.5 & 9.1 \\
\hline $7 / 28 / 1999$ & $15: 30: 40$ & 28.16 & 418 & 92 & 7.2 & 9.1 \\
\hline $7 / 28 / 1999$ & $16: 00: 40$ & 27.95 & 419 & 88.8 & 7.0 & 9.1 \\
\hline 7/28/1999 & $16: 30: 40$ & 27.73 & 421 & 85.7 & 6.7 & 9.1 \\
\hline $7 / 28 / 1999$ & $17: 00: 40$ & 27.55 & 419 & 84.5 & 6.7 & 9.0 \\
\hline $7 / 28 / 1999$ & $17: 30: 40$ & 27.46 & 419 & 86.2 & 6.8 & 9.0 \\
\hline $7 / 28 / 1999$ & 18:00:40 & 27.48 & 418 & 91 & 7.2 & 9.0 \\
\hline $7 / 28 / 1999$ & 18:30:40 & 27.48 & 417 & 93.5 & 7.4 & 9.0 \\
\hline $7 / 28 / 1999$ & 19:00:40 & 27.51 & 417 & 95.5 & 7.5 & 9.1 \\
\hline $7 / 28 / 1999$ & $19: 30: 40$ & 27.57 & 416 & 98.4 & 7.8 & 9.1 \\
\hline 7/28/1999 & 20:00:40 & 27.59 & 416 & 99.7 & 7.9 & 9.1 \\
\hline $7 / 28 / 1999$ & $20: 30: 40$ & 27.55 & 416 & 98.8 & 7.8 & 9.1 \\
\hline 7/28/1999 & 21:00:40 & 27.46 & 415 & 97.8 & 7.7 & 9.1 \\
\hline $7 / 28 / 1999$ & 21:30:40 & 27.38 & 415 & 96.5 & 7.6 & 9.1 \\
\hline $7 / 28 / 1999$ & $22: 00: 40$ & 27.31 & 414 & 95.4 & 7.6 & 9.1 \\
\hline $7 / 28 / 1999$ & $22: 30: 40$ & 27.25 & 414 & 94.6 & 7.5 & 9.1 \\
\hline $7 / 28 / 1999$ & 23:00:40 & 27.15 & 413 & 93.3 & 7.4 & 9.1 \\
\hline $7 / 28 / 1999$ & $23: 30: 40$ & 27.09 & 412 & 92.1 & 7.3 & 9.1 \\
\hline $7 / 29 / 1999$ & 0:00:40 & 27.01 & 411 & 91.3 & 7.3 & 9.1 \\
\hline 7/29/1999 & $0: 30: 40$ & 26.92 & 410 & 90.2 & 7.2 & 9.2 \\
\hline $7 / 29 / 1999$ & 1:00:40 & 26.83 & 409 & 89.1 & 7.1 & 9.2 \\
\hline $7 / 29 / 1999$ & $1: 30: 40$ & 26.74 & 408 & 88.2 & 7.1 & 9.2 \\
\hline $7 / 29 / 1999$ & $2: 00: 40$ & 26.63 & 407 & 86.7 & 7.0 & 9.2 \\
\hline 7/29/1999 & $2: 30: 40$ & 26.52 & 407 & 85.3 & 6.9 & 9.2 \\
\hline $7 / 29 / 1999$ & $3: 00: 40$ & 26.4 & 406 & 83.6 & 6.7 & 9.2 \\
\hline $7 / 29 / 1999$ & $3: 30: 40$ & 26.28 & 406 & 81.9 & 6.6 & 9.2 \\
\hline 7/29/1999 & 4:00:40 & 26.18 & 406 & 80.1 & 6.5 & 9.2 \\
\hline $7 / 29 / 1999$ & 4:30:40 & 26.07 & 406 & 78.3 & 6.3 & 9.2 \\
\hline $7 / 29 / 1999$ & 5:00:40 & 25.97 & 406 & 76.5 & 6.2 & 9.2 \\
\hline 7/29/1999 & $5: 30: 40$ & 25.87 & 406 & 74.6 & 6.1 & 9.2 \\
\hline 7/29/1999 & 6:00:40 & 25.77 & 406 & 73 & 5.9 & 9.2 \\
\hline 7/29/1999 & $6: 30: 40$ & 25.68 & 406 & 71.5 & 5.8 & 9.2 \\
\hline 7/29/1999 & 7:00:40 & 25.56 & 406 & 70.4 & 5.8 & 9.2 \\
\hline
\end{tabular}


78 Water-Quality Synoptic Sampling, July 1999: North Fork Shenandoah River, Virginia

Appendix Continued

\begin{tabular}{|c|c|c|c|c|c|c|}
\hline Date & Time & $\begin{array}{c}\text { Water } \\
\text { temperature } \\
\text { ('C) }\end{array}$ & $\begin{array}{c}\text { SC } \\
(\mu \mathrm{S} / \mathrm{cm})\end{array}$ & $\begin{array}{c}\text { D0sat } \\
\text { (percent) }\end{array}$ & $\begin{array}{c}\text { DO } \\
\text { (mg/L) }\end{array}$ & $\begin{array}{c}\text { pH } \\
\text { (standard } \\
\text { units) }\end{array}$ \\
\hline $7 / 29 / 1999$ & $7: 30: 40$ & 25.46 & 406 & 69.6 & $5.7^{\mathbf{b}}$ & 9.2 \\
\hline $7 / 29 / 1999$ & 8:00:40 & 25.38 & 406 & 70.5 & 5.8 & 9.2 \\
\hline $7 / 29 / 1999$ & 8:30:40 & 25.29 & 406 & 70.7 & 5.8 & 9.2 \\
\hline \multicolumn{7}{|c|}{ Site 49} \\
\hline $7 / 27 / 1999$ & $15: 30: 40$ & 28.25 & 406 & 107.5 & 8.4 & 9.0 \\
\hline $7 / 27 / 1999$ & $16: 00: 40$ & 28.32 & 407 & 108.6 & 8.5 & 9.0 \\
\hline $7 / 27 / 1999$ & $16: 30: 40$ & 28.35 & 407 & 110.3 & 8.6 & 9.0 \\
\hline $7 / 27 / 1999$ & $17: 00: 40$ & 28.34 & 411 & 110.5 & 8.6 & 9.0 \\
\hline $7 / 27 / 1999$ & $17: 30: 40$ & 28.18 & 411 & 108.7 & 8.5 & 9.0 \\
\hline $7 / 27 / 1999$ & 18:00:40 & 28.23 & 415 & 107.7 & 8.4 & 9.0 \\
\hline $7 / 27 / 1999$ & 18:30:40 & 28.22 & 414 & 107.3 & 8.4 & 9.0 \\
\hline $7 / 27 / 1999$ & 19:00:40 & 28.28 & 416 & 107.5 & 8.4 & 9.0 \\
\hline $7 / 27 / 1999$ & $19: 30: 40$ & 28.22 & 410 & 107.5 & 8.4 & 8.9 \\
\hline $7 / 27 / 1999$ & $20: 00: 40$ & 28.3 & 414 & 107.5 & 8.4 & 9.0 \\
\hline $7 / 27 / 1999$ & $20: 30: 40$ & 28.28 & 412 & 106.5 & 8.3 & 9.0 \\
\hline $7 / 27 / 1999$ & 21:00:40 & 28.27 & 412 & 105 & 8.2 & 9.0 \\
\hline $7 / 27 / 1999$ & $21: 30: 40$ & 28.2 & 410 & 103.4 & 8.1 & 9.0 \\
\hline $7 / 27 / 1999$ & 22:00:40 & 28.13 & 409 & 102.2 & 8.0 & 9.0 \\
\hline $7 / 27 / 1999$ & $22: 30: 40$ & 28.05 & 408 & 101.7 & 8.0 & 9.0 \\
\hline $7 / 27 / 1999$ & $23: 00: 40$ & 27.98 & 408 & 101.3 & 7.9 & 9.0 \\
\hline $7 / 27 / 1999$ & $23: 30: 40$ & 27.9 & 407 & 100.9 & 7.9 & 9.0 \\
\hline $7 / 28 / 1999$ & 0:00:40 & 27.8 & 406 & 100.3 & 7.9 & 9.0 \\
\hline $7 / 28 / 1999$ & $0: 30: 40$ & 27.7 & 405 & 99.9 & 7.9 & 9.0 \\
\hline $7 / 28 / 1999$ & 1:00:40 & 27.63 & 404 & 99.7 & 7.9 & 9.1 \\
\hline $7 / 28 / 1999$ & $1: 30: 40$ & 27.52 & 403 & 98.5 & 7.8 & 9.1 \\
\hline $7 / 28 / 1999$ & 2:00:40 & 27.41 & 402 & 97.2 & 7.7 & 9.1 \\
\hline $7 / 28 / 1999$ & 2:30:40 & 27.32 & 402 & 96 & 7.6 & 9.1 \\
\hline $7 / 28 / 1999$ & 3:00:40 & 27.23 & 402 & 94.5 & 7.5 & 9.1 \\
\hline $7 / 28 / 1999$ & $3: 30: 40$ & 27.11 & 401 & 92.4 & 7.3 & 9.1 \\
\hline $7 / 28 / 1999$ & 4:00:40 & 27.01 & 401 & 90.4 & 7.2 & 9.1 \\
\hline $7 / 28 / 1999$ & 4:30:40 & 26.91 & 401 & 88.5 & 7.1 & 9.1 \\
\hline $7 / 28 / 1999$ & 5:00:40 & 26.79 & 401 & 86.3 & 6.9 & 9.1 \\
\hline $7 / 28 / 1999$ & $5: 30: 40$ & 26.66 & 400 & 84.1 & 6.7 & 9.1 \\
\hline $7 / 28 / 1999$ & 6:00:40 & 26.55 & 400 & 81.9 & 6.6 & 9.1 \\
\hline $7 / 28 / 1999$ & $6: 30: 40$ & 26.43 & 400 & 79.6 & 6.4 & 9.1 \\
\hline $7 / 28 / 1999$ & 7:00:40 & 26.29 & 400 & 78.1 & 6.3 & 9.1 \\
\hline $7 / 28 / 1999$ & 7:30:40 & 26.19 & 400 & 77 & 6.2 & 9.1 \\
\hline $7 / 28 / 1999$ & 8:00:40 & 26.18 & 401 & 77.4 & 6.3 & 9.1 \\
\hline $7 / 28 / 1999$ & 8:30:40 & 26.16 & 400 & 78.8 & 6.4 & 9.1 \\
\hline $7 / 28 / 1999$ & 9:00:40 & 26.22 & 400 & 80.9 & 6.5 & 9.1 \\
\hline $7 / 28 / 1999$ & 9:30:40 & 26.34 & 399 & 84.1 & 6.8 & 9.1 \\
\hline $7 / 28 / 1999$ & 10:00:40 & 26.5 & 399 & 87.4 & 7.0 & 9.1 \\
\hline $7 / 28 / 1999$ & $10: 30: 40$ & 26.74 & 399 & 91.3 & 7.3 & 9.1 \\
\hline $7 / 28 / 1999$ & 11:00:40 & 27.05 & 398 & 96.1 & 7.6 & 9.2 \\
\hline $7 / 28 / 1999$ & 11:30:40 & 27.35 & 398 & 101.2 & 8.0 & 9.2 \\
\hline $7 / 28 / 1999$ & $12: 00: 40$ & 27.74 & 398 & 106.1 & 8.3 & 9.2 \\
\hline $7 / 28 / 1999$ & $12: 30: 40$ & 28.13 & 397 & 110.6 & 8.6 & 9.2 \\
\hline $7 / 28 / 1999$ & 13:00:40 & 28.38 & 397 & 113.1 & $8.8^{\mathbf{a}}$ & 9.2 \\
\hline $7 / 28 / 1999$ & $13: 30: 40$ & 28.45 & 398 & 113 & 8.8 & 9.1 \\
\hline
\end{tabular}


Appendix Continued

\begin{tabular}{|c|c|c|c|c|c|c|}
\hline Date & Time & $\begin{array}{c}\text { Water } \\
\text { temperature } \\
\text { ('C) }\end{array}$ & $\begin{array}{c}\text { SC } \\
(\mu \mathrm{S} / \mathrm{cm})\end{array}$ & $\begin{array}{c}\text { DOsat } \\
\text { (percent) }\end{array}$ & $\begin{array}{c}\mathrm{DO} \\
\text { (mg/L) }\end{array}$ & $\begin{array}{c}\text { pH } \\
\text { (standard } \\
\text { units) }\end{array}$ \\
\hline $7 / 28 / 1999$ & $14: 00: 40$ & 28.49 & 399 & 112.1 & 8.7 & 9.1 \\
\hline $7 / 28 / 1999$ & $14: 30: 40$ & 28.32 & 398 & 109.9 & 8.5 & 9.1 \\
\hline $7 / 28 / 1999$ & 15:00:40 & 28.08 & 399 & 106.5 & 8.3 & 9.1 \\
\hline $7 / 28 / 1999$ & $15: 30: 40$ & 27.92 & 401 & 101 & 7.9 & 9.0 \\
\hline $7 / 28 / 1999$ & 16:00:40 & 27.72 & 402 & 97.2 & 7.6 & 9.0 \\
\hline $7 / 28 / 1999$ & $16: 30: 40$ & 27.56 & 403 & 93.2 & 7.4 & 9.0 \\
\hline $7 / 28 / 1999$ & 17:00:40 & 27.36 & 404 & 90 & 7.1 & 9.0 \\
\hline $7 / 28 / 1999$ & $17: 30: 40$ & 27.25 & 403 & 89.4 & 7.1 & 9.0 \\
\hline $7 / 28 / 1999$ & 18:00:40 & 27.16 & 401 & 90.6 & 7.2 & 8.9 \\
\hline $7 / 28 / 1999$ & 18:30:40 & 27.18 & 403 & 92.1 & 7.3 & 9.0 \\
\hline $7 / 28 / 1999$ & 19:00:40 & 27.14 & 400 & 93.9 & 7.5 & 9.0 \\
\hline $7 / 28 / 1999$ & $19: 30: 40$ & 27.19 & 401 & 96.8 & 7.7 & 9.0 \\
\hline $7 / 28 / 1999$ & 20:00:40 & 27.18 & 400 & 98.1 & 7.8 & 9.0 \\
\hline $7 / 28 / 1999$ & $20: 30: 40$ & 27.14 & 400 & 97.8 & 7.8 & 9.0 \\
\hline $7 / 28 / 1999$ & 21:00:40 & 27.06 & 399 & 96.9 & 7.7 & 9.0 \\
\hline $7 / 28 / 1999$ & $21: 30: 40$ & 26.97 & 399 & 95.7 & 7.6 & 9.0 \\
\hline $7 / 28 / 1999$ & 22:00:40 & 26.86 & 399 & 94.4 & 7.5 & 9.0 \\
\hline $7 / 28 / 1999$ & $22: 30: 40$ & 26.78 & 399 & 93 & 7.4 & 9.0 \\
\hline $7 / 28 / 1999$ & $23: 00: 40$ & 26.71 & 399 & 92 & 7.4 & 9.0 \\
\hline $7 / 28 / 1999$ & $23: 30: 40$ & 26.59 & 398 & 90.6 & 7.3 & 9.0 \\
\hline 7/29/1999 & 0:00:40 & 26.52 & 398 & 89.7 & 7.2 & 9.0 \\
\hline $7 / 29 / 1999$ & $0: 30: 40$ & 26.41 & 396 & 88.6 & 7.1 & 9.0 \\
\hline $7 / 29 / 1999$ & 1:00:40 & 26.32 & 396 & 87.6 & 7.1 & 9.0 \\
\hline $7 / 29 / 1999$ & $1: 30: 40$ & 26.24 & 395 & 86.7 & 7.0 & 9.1 \\
\hline $7 / 29 / 1999$ & 2:00:40 & 26.12 & 394 & 85.5 & 6.9 & 9.1 \\
\hline $7 / 29 / 1999$ & $2: 30: 40$ & 26.03 & 394 & 84.4 & 6.8 & 9.1 \\
\hline $7 / 29 / 1999$ & 3:00:40 & 25.9 & 394 & 83.2 & 6.8 & 9.1 \\
\hline $7 / 29 / 1999$ & $3: 30: 40$ & 25.79 & 394 & 81.7 & 6.7 & 9.1 \\
\hline $7 / 29 / 1999$ & 4:00:40 & 25.69 & 394 & 80.4 & 6.6 & 9.1 \\
\hline 7/29/1999 & 4:30:40 & 25.6 & 395 & 79 & 6.5 & 9.1 \\
\hline $7 / 29 / 1999$ & 5:00:40 & 25.47 & 394 & 77.4 & 6.3 & 9.1 \\
\hline $7 / 29 / 1999$ & $5: 30: 40$ & 25.36 & 394 & 76 & 6.2 & 9.1 \\
\hline 7/29/1999 & $6: 00: 40$ & 25.3 & 395 & 74.7 & 6.1 & 9.1 \\
\hline 7/29/1999 & $6: 30: 40$ & 25.2 & 396 & 73.5 & 6.1 & 9.1 \\
\hline $7 / 29 / 1999$ & 7:00:40 & 25.07 & 395 & 72.7 & 6.0 & 9.1 \\
\hline $7 / 29 / 1999$ & $7: 30: 40$ & 24.96 & 395 & 71.9 & $5.9^{\mathbf{b}}$ & 9.1 \\
\hline $7 / 29 / 1999$ & 8:00:40 & 24.93 & 396 & 71.9 & 5.9 & 9.1 \\
\hline 7/29/1999 & $8: 30: 40$ & 24.84 & 396 & 72.2 & 6.0 & 9.1 \\
\hline \multicolumn{7}{|c|}{ Site 50} \\
\hline $7 / 28 / 1999$ & 14:00:40 & 27.37 & 405 & 85.9 & 6.8 & 9.0 \\
\hline $7 / 28 / 1999$ & $14: 30: 40$ & 27.38 & 404 & 83.3 & 6.6 & 9.0 \\
\hline $7 / 28 / 1999$ & $15: 00: 40$ & 27.37 & 403 & 83.6 & 6.6 & 9.0 \\
\hline $7 / 28 / 1999$ & $15: 30: 40$ & 27.36 & 403 & 84 & 6.6 & 9.0 \\
\hline $7 / 28 / 1999$ & $16: 00: 40$ & 27.35 & 403 & 85.2 & 6.7 & 9.1 \\
\hline $7 / 28 / 1999$ & $16: 30: 40$ & 27.37 & 402 & 88.4 & 7.0 & 9.1 \\
\hline $7 / 28 / 1999$ & 17:00:40 & 27.4 & 400 & 92.5 & 7.3 & 9.1 \\
\hline $7 / 28 / 1999$ & $17: 30: 40$ & 27.5 & 398 & 97.8 & 7.7 & 9.1 \\
\hline $7 / 28 / 1999$ & 18:00:40 & 27.6 & 397 & 102.3 & 8.1 & 9.1 \\
\hline $7 / 28 / 1999$ & 18:30:40 & 27.63 & 398 & 102.5 & 8.1 & 9.1 \\
\hline
\end{tabular}


Water-Quality Synoptic Sampling, July 1999: North Fork Shenandoah River, Virginia

Appendix Continued

\begin{tabular}{|c|c|c|c|c|c|c|}
\hline Date & Time & $\begin{array}{c}\text { Water } \\
\text { temperature } \\
\text { ('C) }\end{array}$ & $\begin{array}{c}\text { SC } \\
(\mu \mathbf{S} / \mathbf{c m})\end{array}$ & $\begin{array}{c}\text { D0sat } \\
\text { (percent) }\end{array}$ & $\begin{array}{c}\text { DO } \\
\text { (mg/L) }\end{array}$ & $\begin{array}{c}\text { pH } \\
\text { (standard } \\
\text { units) }\end{array}$ \\
\hline $7 / 28 / 1999$ & 19:00:40 & 27.69 & 397 & 104.6 & 8.2 & 9.1 \\
\hline 7/28/1999 & 19:30:40 & 27.76 & 396 & 107.8 & 8.5 & 9.2 \\
\hline $7 / 28 / 1999$ & 20:00:40 & 27.84 & 394 & 111.1 & 8.7 & 9.2 \\
\hline $7 / 28 / 1999$ & $20: 30: 40$ & 27.89 & 394 & 112.6 & 8.8 & 9.2 \\
\hline $7 / 28 / 1999$ & 21:00:40 & 27.94 & 393 & 114.7 & 9.0 & 9.2 \\
\hline $7 / 28 / 1999$ & $21: 30: 40$ & 27.94 & 391 & 116.1 & 9.1 & 9.2 \\
\hline 7/28/1999 & $22: 00: 40$ & 27.87 & 391 & 115.4 & 9.1 & 9.2 \\
\hline $7 / 28 / 1999$ & $22: 30: 40$ & 27.77 & 392 & 112.3 & 8.8 & 9.2 \\
\hline $7 / 28 / 1999$ & 23:00:40 & 27.67 & 392 & 109.4 & 8.6 & 9.2 \\
\hline 7/28/1999 & $23: 30: 40$ & 27.5 & 393 & 104.8 & 8.3 & 9.2 \\
\hline $7 / 29 / 1999$ & 0:00:40 & 27.34 & 394 & 100.4 & 8.0 & 9.1 \\
\hline $7 / 29 / 1999$ & 0:30:40 & 27.22 & 395 & 97.7 & 7.8 & 9.1 \\
\hline $7 / 29 / 1999$ & 1:00:40 & 27.07 & 395 & 95.3 & 7.6 & 9.1 \\
\hline $7 / 29 / 1999$ & $1: 30: 40$ & 26.93 & 396 & 91.5 & 7.3 & 9.1 \\
\hline $7 / 29 / 1999$ & 2:00:40 & 26.77 & 397 & 87.7 & 7.0 & 9.1 \\
\hline $7 / 29 / 1999$ & 2:30:40 & 26.59 & 398 & 84.2 & 6.8 & 9.0 \\
\hline $7 / 29 / 1999$ & 3:00:40 & 26.46 & 399 & 82.1 & 6.6 & 9.0 \\
\hline $7 / 29 / 1999$ & $3: 30: 40$ & 26.28 & 400 & 79.1 & 6.4 & 9.0 \\
\hline $7 / 29 / 1999$ & 4:00:40 & 26.18 & 401 & 77 & 6.2 & 9.0 \\
\hline $7 / 29 / 1999$ & 4:30:40 & 26.04 & 402 & 74.9 & 6.1 & 9.0 \\
\hline $7 / 29 / 1999$ & 5:00:40 & 25.9 & 403 & 72.7 & 5.9 & 8.9 \\
\hline $7 / 29 / 1999$ & $5: 30: 40$ & 25.78 & 402 & 71.5 & 5.8 & 8.9 \\
\hline $7 / 29 / 1999$ & 6:00:40 & 25.63 & 402 & 70 & 5.7 & 8.9 \\
\hline $7 / 29 / 1999$ & $6: 30: 40$ & 25.54 & 402 & 68.9 & 5.6 & 8.9 \\
\hline $7 / 29 / 1999$ & 7:00:40 & 25.41 & 402 & 68.5 & 5.6 & 8.9 \\
\hline $7 / 29 / 1999$ & 7:30:40 & 25.31 & 401 & 67.4 & 5.5 & 8.9 \\
\hline $7 / 29 / 1999$ & 8:00:40 & 25.24 & 401 & 67.2 & 5.5 & 8.9 \\
\hline $7 / 29 / 1999$ & 8:30:40 & 25.15 & 400 & 66.9 & 5.5 & 8.9 \\
\hline $7 / 29 / 1999$ & 9:00:40 & 25.16 & 400 & 66.7 & 5.5 & 8.9 \\
\hline $7 / 29 / 1999$ & $9: 30: 40$ & 25.23 & 402 & 66.8 & 5.5 & 8.9 \\
\hline $7 / 29 / 1999$ & 10:00:40 & 25.39 & 403 & 66.8 & $5.5^{\mathbf{b}}$ & 8.9 \\
\hline $7 / 29 / 1999$ & $10: 30: 40$ & 25.4 & 402 & 68.5 & 5.6 & 8.9 \\
\hline $7 / 29 / 1999$ & 11:00:40 & 25.58 & 401 & 70.4 & 5.8 & 8.9 \\
\hline 7/29/1999 & $11: 30: 40$ & 25.7 & 400 & 71.8 & 5.9 & 8.9 \\
\hline $7 / 29 / 1999$ & $12: 00: 40$ & 25.93 & 398 & 72.6 & 5.9 & 9.0 \\
\hline $7 / 29 / 1999$ & $12: 30: 40$ & 26.27 & 398 & 74.1 & 6.0 & 9.0 \\
\hline $7 / 29 / 1999$ & 13:00:40 & 26.3 & 397 & 74.5 & 6.0 & 9.0 \\
\hline $7 / 29 / 1999$ & $13: 30: 40$ & 26.52 & 397 & 76.2 & 6.1 & 9.0 \\
\hline $7 / 29 / 1999$ & 14:00:40 & 26.62 & 396 & 77 & 6.2 & 9.0 \\
\hline $7 / 29 / 1999$ & $14: 30: 40$ & 26.74 & 396 & 78.1 & 6.3 & 9.0 \\
\hline $7 / 29 / 1999$ & 15:00:40 & 26.89 & 396 & 80.6 & 6.4 & 9.0 \\
\hline $7 / 29 / 1999$ & $15: 30: 40$ & 26.95 & 395 & 83.3 & 6.6 & 9.0 \\
\hline $7 / 29 / 1999$ & $16: 00: 40$ & 27.06 & 394 & 87 & 6.9 & 9.0 \\
\hline $7 / 29 / 1999$ & $16: 30: 40$ & 27.09 & 394 & 91.1 & 7.2 & 9.0 \\
\hline $7 / 29 / 1999$ & 17:00:40 & 27.2 & 393 & 94.9 & 7.5 & 9.1 \\
\hline $7 / 29 / 1999$ & $17: 30: 40$ & 27.37 & 393 & 100.2 & 7.9 & 9.1 \\
\hline $7 / 29 / 1999$ & 18:00:40 & 27.47 & 392 & 105.2 & 8.3 & 9.1 \\
\hline $7 / 29 / 1999$ & $18: 30: 40$ & 27.55 & 391 & 109.5 & 8.6 & 9.1 \\
\hline $7 / 29 / 1999$ & 19:00:40 & 27.63 & 391 & 113 & 8.9 & 9.1 \\
\hline $7 / 29 / 1999$ & $19: 30: 40$ & 27.66 & 391 & 114.1 & 9.0 & 9.1 \\
\hline
\end{tabular}


Appendix Continued

\begin{tabular}{|c|c|c|c|c|c|c|}
\hline Date & Time & $\begin{array}{c}\text { Water } \\
\text { temperature } \\
\text { ('C) }\end{array}$ & $\begin{array}{c}\text { SC } \\
(\mu \mathrm{S} / \mathrm{cm})\end{array}$ & $\begin{array}{c}\text { DOsat } \\
\text { (percent) }\end{array}$ & $\begin{array}{c}\text { DO } \\
(\mathrm{mg} / \mathrm{L})\end{array}$ & $\begin{array}{c}\mathrm{pH} \\
\text { (standard } \\
\text { units) }\end{array}$ \\
\hline $7 / 29 / 1999$ & $20: 00: 40$ & 27.71 & 391 & 115.7 & 9.1 & 9.1 \\
\hline $7 / 29 / 1999$ & $20: 30: 40$ & 27.74 & 391 & 117.2 & 9.2 & 9.1 \\
\hline $7 / 29 / 1999$ & 21:00:40 & 27.8 & 390 & 119.7 & 9.4 & 9.1 \\
\hline $7 / 29 / 1999$ & $21: 30: 40$ & 27.81 & 390 & 123 & $9.7^{\mathbf{a}}$ & 9.1 \\
\hline $7 / 29 / 1999$ & $22: 00: 40$ & 27.76 & 390 & 122.8 & 9.6 & 9.1 \\
\hline $7 / 29 / 1999$ & $22: 30: 40$ & 27.71 & 391 & 122 & 9.6 & 9.1 \\
\hline $7 / 29 / 1999$ & $23: 00: 40$ & 27.64 & 392 & 121 & 9.5 & 9.1 \\
\hline $7 / 29 / 1999$ & $23: 30: 40$ & 27.57 & 393 & 119.6 & 9.4 & 9.1 \\
\hline 7/30/1999 & 0:00:40 & 27.51 & 394 & 118.3 & 9.3 & 9.1 \\
\hline $7 / 30 / 1999$ & 0:30:40 & 27.44 & 395 & 117.1 & 9.3 & 9.1 \\
\hline $7 / 30 / 1999$ & 1:00:40 & 27.32 & 396 & 115.8 & 9.2 & 9.1 \\
\hline $7 / 30 / 1999$ & $1: 30: 40$ & 27.18 & 397 & 113.3 & 9.0 & 9.0 \\
\hline $7 / 30 / 1999$ & 2:00:40 & 27.07 & 399 & 110.4 & 8.8 & 9.0 \\
\hline $7 / 30 / 1999$ & $2: 30: 40$ & 26.97 & 399 & 107.8 & 8.6 & 9.0 \\
\hline $7 / 30 / 1999$ & 3:00:40 & 26.85 & 400 & 104.5 & 8.3 & 9.0 \\
\hline $7 / 30 / 1999$ & $3: 30: 40$ & 26.77 & 401 & 101.5 & 8.1 & 9.0 \\
\hline $7 / 30 / 1999$ & 4:00:40 & 26.63 & 402 & 97.9 & 7.9 & 9.0 \\
\hline 7/30/1999 & 4:30:40 & 26.49 & 402 & 93.6 & 7.5 & 9.0 \\
\hline $7 / 30 / 1999$ & 5:00:40 & 26.33 & 403 & 89.1 & 7.2 & 8.9 \\
\hline $7 / 30 / 1999$ & $5: 30: 40$ & 26.19 & 403 & 85.3 & 6.9 & 8.9 \\
\hline $7 / 30 / 1999$ & 6:00:40 & 26.02 & 404 & 80.7 & 6.5 & 8.9 \\
\hline $7 / 30 / 1999$ & $6: 30: 40$ & 25.93 & 403 & 78 & 6.3 & 8.9 \\
\hline $7 / 30 / 1999$ & 7:00:40 & 25.74 & 403 & 74 & 6.0 & 8.8 \\
\hline 7/30/1999 & 7:30:40 & 25.71 & 402 & 73 & 6.0 & 8.8 \\
\hline 7/30/1999 & 8:00:40 & 25.7 & 401 & 72.4 & 5.9 & 8.8 \\
\hline $7 / 30 / 1999$ & $8: 30: 40$ & 25.64 & 401 & 71.4 & 5.8 & 8.8 \\
\hline \multicolumn{7}{|c|}{ Site 51} \\
\hline $7 / 29 / 1999$ & $9: 30: 40$ & 25.39 & 402 & 78.3 & 6.4 & 8.9 \\
\hline $7 / 29 / 1999$ & 10:00:40 & 25.46 & 402 & 77.9 & $6.4^{\mathbf{b}}$ & 8.9 \\
\hline 7/29/1999 & $10: 30: 40$ & 25.65 & 402 & 79.9 & 6.5 & 8.9 \\
\hline $7 / 29 / 1999$ & 11:00:40 & 25.85 & 402 & 82.2 & 6.7 & 8.9 \\
\hline $7 / 29 / 1999$ & $11: 30: 40$ & 26.04 & 401 & 84.1 & 6.8 & 8.9 \\
\hline $7 / 29 / 1999$ & $12: 00: 40$ & 26.29 & 401 & 86.5 & 7.0 & 8.9 \\
\hline $7 / 29 / 1999$ & $12: 30: 40$ & 26.3 & 400 & 85.3 & 6.9 & 8.9 \\
\hline $7 / 29 / 1999$ & $13: 00: 40$ & 26.55 & 399 & 89.1 & 7.2 & 9.0 \\
\hline $7 / 29 / 1999$ & $13: 30: 40$ & 26.75 & 398 & 89.8 & 7.2 & 9.0 \\
\hline $7 / 29 / 1999$ & 14:00:40 & 26.87 & 397 & 92.4 & 7.4 & 9.0 \\
\hline $7 / 29 / 1999$ & $14: 30: 40$ & 26.89 & 397 & 91.3 & 7.3 & 9.0 \\
\hline $7 / 29 / 1999$ & $15: 00: 40$ & 27.04 & 397 & 94.4 & 7.5 & 9.0 \\
\hline $7 / 29 / 1999$ & $15: 30: 40$ & 27.07 & 396 & 94.9 & 7.6 & 9.0 \\
\hline $7 / 29 / 1999$ & $16: 00: 40$ & 27.08 & 396 & 95.5 & 7.6 & 9.0 \\
\hline $7 / 29 / 1999$ & $16: 30: 40$ & 27.08 & 395 & 97.1 & 7.7 & 9.0 \\
\hline $7 / 29 / 1999$ & $17: 00: 40$ & 27.25 & 395 & 102.5 & 8.1 & 9.0 \\
\hline $7 / 29 / 1999$ & $17: 30: 40$ & 27.33 & 394 & 104.9 & 8.3 & 9.1 \\
\hline $7 / 29 / 1999$ & $18: 00: 40$ & 27.41 & 394 & 106.3 & 8.4 & 9.1 \\
\hline $7 / 29 / 1999$ & $18: 30: 40$ & 27.44 & 393 & 107 & 8.5 & 9.1 \\
\hline $7 / 29 / 1999$ & 19:00:40 & 27.52 & 393 & 108.6 & 8.6 & 9.1 \\
\hline 7/29/1999 & 19:30:40 & 27.61 & 392 & 110.4 & 8.7 & 9.1 \\
\hline 7/29/1999 & 20:00:40 & 27.67 & 392 & 110.8 & 8.7 & 9.1 \\
\hline
\end{tabular}


82 Water-Quality Synoptic Sampling, July 1999: North Fork Shenandoah River, Virginia

Appendix Continued

\begin{tabular}{|c|c|c|c|c|c|c|}
\hline Date & Time & $\begin{array}{c}\text { Water } \\
\text { temperature } \\
\text { ('C) }\end{array}$ & $\begin{array}{c}\text { SC } \\
(\mu \mathbf{S} / \mathbf{c m})\end{array}$ & $\begin{array}{c}\text { DOsat } \\
\text { (percent) }\end{array}$ & $\begin{array}{c}\text { DO } \\
\text { (mg/L) }\end{array}$ & $\begin{array}{c}\text { pH } \\
\text { (standard } \\
\text { units) }\end{array}$ \\
\hline $7 / 29 / 1999$ & $20: 30: 40$ & 27.7 & 392 & 111.2 & 8.8 & 9.1 \\
\hline $7 / 29 / 1999$ & 21:00:40 & 27.7 & 392 & 111.7 & 8.8 & 9.1 \\
\hline $7 / 29 / 1999$ & $21: 30: 40$ & 27.72 & 392 & 112.4 & 8.8 & 9.1 \\
\hline $7 / 29 / 1999$ & $22: 00: 40$ & 27.74 & 392 & 113.3 & 8.9 & 9.1 \\
\hline $7 / 29 / 1999$ & $22: 30: 40$ & 27.73 & 392 & 113.5 & $8.9^{\mathbf{a}}$ & 9.1 \\
\hline $7 / 29 / 1999$ & $23: 00: 40$ & 27.7 & 392 & 113.3 & 8.9 & 9.1 \\
\hline $7 / 29 / 1999$ & $23: 30: 40$ & 27.65 & 393 & 112.8 & 8.9 & 9.1 \\
\hline 7/30/1999 & 0:00:40 & 27.59 & 394 & 112.1 & 8.8 & 9.1 \\
\hline $7 / 30 / 1999$ & $0: 30: 40$ & 27.52 & 395 & 111.3 & 8.8 & 9.1 \\
\hline 7/30/1999 & 1:00:40 & 27.45 & 396 & 110.4 & 8.7 & 9.1 \\
\hline 7/30/1999 & 1:30:40 & 27.35 & 397 & 108.8 & 8.6 & 9.1 \\
\hline 7/30/1999 & 2:00:40 & 27.26 & 398 & 107.1 & 8.5 & 9.1 \\
\hline 7/30/1999 & 2:30:40 & 27.15 & 399 & 105.2 & 8.4 & 9.0 \\
\hline 7/30/1999 & 3:00:40 & 27.03 & 399 & 103 & 8.2 & 9.0 \\
\hline 7/30/1999 & 3:30:40 & 26.9 & 401 & 100.4 & 8.0 & 9.0 \\
\hline $7 / 30 / 1999$ & 4:00:40 & 26.76 & 402 & 97.4 & 7.8 & 9.0 \\
\hline 7/30/1999 & 4:30:40 & 26.61 & 403 & 94.1 & 7.5 & 8.9 \\
\hline $7 / 30 / 1999$ & 5:00:40 & 26.46 & 403 & 90.8 & 7.3 & 8.9 \\
\hline $7 / 30 / 1999$ & $5: 30: 40$ & 26.31 & 404 & 87.6 & 7.1 & 8.9 \\
\hline 7/30/1999 & 6:00:40 & 26.18 & 404 & 84.9 & 6.9 & 8.9 \\
\hline 7/30/1999 & 6:30:40 & 26.06 & 404 & 82.5 & 6.7 & 8.8 \\
\hline 7/30/1999 & 7:00:40 & 25.97 & 404 & 81.2 & 6.6 & 8.8 \\
\hline 7/30/1999 & 7:30:40 & 25.92 & 404 & 81.1 & 6.6 & 8.8 \\
\hline 7/30/1999 & 8:00:40 & 25.89 & 403 & 80.2 & 6.5 & 8.8 \\
\hline $7 / 30 / 1999$ & $8: 30: 40$ & 25.84 & 403 & 79.5 & 6.5 & 8.8 \\
\hline $7 / 30 / 1999$ & 9:00:40 & 25.85 & 402 & 80.4 & 6.5 & 8.8 \\
\hline
\end{tabular}

${ }^{\mathrm{a}}$ Maximum dissolved-oxygen concentration

${ }^{b}$ Minimum dissolved-oxygen concentration 